\title{
Family Members, Not Workers
}

The Experiences of Statutory Family Care Helpers (FCHs) in South Korea

\author{
Dissertation \\ zur Erlangung des Doktorgrades \\ der Sozialwissenschaftlichen Fakultät \\ der Georg-August-Universität Göttingen
}

vorgelegt von

Tae-Young Yun

geboren in Gimcheon (Südkorea)

Göttingen, 2019 
Erstgutachterin: Prof. Dr. Ilona Ostner

Zweitgutachterin: Prof. Dr. Gabriele Rosenthal

Drittgutachter: Prof. Dr. Steffen Kühnel

Tag der mündlichen Prüfung: 12. 02.2019 
For my mother Suk, who made me keep going 


\section{Abbreviations}

ADL Activities of Daily Living

ALMP Active Labour Market Policy

BOP Basic Old-Age Pension (between 2008 and 2014)

BP $\quad$ Basic Pension (after 2014)

DRD Declaration on the Right to Development

FCH Family Care Helper

GDI Gender-related Development Index

GEM Gender Empowerment Measure

GDP Gross Domestic Product

IADL Instrumental Activities of Daily Living

ICESCR International Covenant on Economic, Social and Cultural Rights

ICCPR International Covenant on Civil and Political Rights

IMF International Monetary Fund

KIHASA Korea Institute for Health and Social Affairs

KLI Korea Labour Institute

KOSTAT Statistics Korea

KRW South Korean Won, the currency of South Korea

KWDI Korean Women's Development Institute

LTC Long-term care

LTCI Long-Term Care Insurance

MOHW Ministry of Health and Welfare (Korea)

NBLS National Basic Livelihood Security Programme

NHIC National Health Insurance Corporation in South Korea (Until 2012)

NHIS National Health Insurance Service in South Korea (after 2013)

OECD Organisation for Economic Co-operation and Development

PCI Problem-Centred Interview

UDHR Universal Declaration of Human Rights

UN United Nations

UNDP United Nations Development Programme

*Exchange rate between the Republic of Korea Won (KRW) and the United States Dollar(s): $1 \mathrm{KRW}=1 \mathrm{USD}$ 


\section{Table of Contents}

\section{CHAPTER I INTRODUCTION}

1.1. Introduction 8

1.2. Aims, Research Questions $\quad 14$

1.3. Approach, Assumptions and the Significance of the Study 16

1.4. Outline of the Chapters 18

CHAPTER II LIVING STATUS IN LATER LIFE AND FAMILY RELATIONS IN KOREA

2.1. Introduction 20

2.2. Living Status in Later Life in Korea $\quad 22$

2.2.1. Old-age income security in Korea $\quad 22$

$\begin{array}{ll}\text { 2.2.2. Housing in later life in Korea } & 29\end{array}$

2.2.3. Health status and social services $\quad 31$

2.3. Family Relations in Korea 33

2.3.1. Changes in family relations in Korea $\quad 35$

2.3.2. Research trends in elderly care practice among families in Korea $\quad 39$

2.4. Family Care Helpers (FCHs) of the LTCI in Korea 43

2.4.1. Family care helpers (FCHs) of the LTCI in Korea 45

2.4.2. Changes in the payments for family care by the LTCI 50

2.5. Conclusion 55

CHAPTER III LITERATURE REVIEW: Sharing Care Responsibilities at Two

\section{Levels}

3.1. Introduction 58

3.2. Care Responsibility, Social Rights, and Social Care 59

3.2.1. Concept of care responsibility $\quad 59$

3.2.2. Social rights and social care $\quad 62$

3.3. Care Arrangements 65

3.3.1. Care arrangements $\quad 65$

3.3.2. Debates on cash for care $\quad 67$

3.3.3. Care culture and the welfare state $\quad 69$

3.4. Family Care as Family Practice $\quad 71$

3.5. Conclusion 75

\section{CHAPTER IV THE METHODS}

4.1. Introduction 78

4.2. Rationale for the Qualitative Approach $\quad 78$

4.3. Research Design and Data Collection $\quad 81$

$\begin{array}{ll}\text { 4.3.1. Preparing PCIs } & 81\end{array}$

4.3.2. Conducting the PCI: the procedure of an interview 86

4.3.3. Ethical considerations for the interviews $\quad 89$

4.4. Data Analysis: from topic-oriented to case reconstructions 91

4.5. Conclusion 98

\section{CHAPTER V RESULT}

5.1. Introduction 99

5.2. Type A: Traditionalists 100

$\begin{array}{ll}\text { 5.2.1. Interview context } & 100\end{array}$ 
5.2.2. Anmi's self-presentation 101

5.2.3. Reconstruction of Anmi’s life story 103

$\begin{array}{ll}\text { 5.2.4. Structural features summary } & 119\end{array}$

5.3. Type B: The Negotiators 125

$\begin{array}{lr}\text { 5.3.1. Interview context } & 126\end{array}$

$\begin{array}{ll}\text { 5.3.2. Self-presentation } & 127\end{array}$

$\begin{array}{ll}\text { 5.3.3. Reconstruction of Sunjoo's life history } & 129\end{array}$

$\begin{array}{ll}\text { 5.3.4. Structural features summery } & 144\end{array}$

5.4. Type C: The Partners 148

$\begin{array}{ll}\text { 5.4.1. Interview context } & 149\end{array}$

$\begin{array}{ll}\text { 5.4.2. Self-presentation } & 149\end{array}$

5.4.3. Reconstruction of Jina's life story 151

5.4.4. Structural features summary 172

5.5. Implications of the Typologies of FCHs 175

\section{CHAPTER VI CONCLUSION}

6.1. Introduction: Aim, Proposition, and Scope of the Research 182

6.2. Summary of the Thesis 183

6.3. The Family's Responsibility for Elderly Care in Social Care 191

6.4. Care Responsibility as a Family Practice 194

6.5. Limitations, and Suggestions on Further Research 197

$\begin{array}{ll}\text { References } & 199\end{array}$

$\begin{array}{ll}\text { Appendix } 1 \text { Contact Letter } & 217\end{array}$

Appendix 2 Interview Consent Form $\quad 219$ 


\section{List of Tables}

Table 2.1. The structure of income sources scheme in later life in Korea 28

Table 2.2. The trends of the elderly households 30

Table 2.3. Major caregivers of the elderly in need of care in Korea 37

Table 2.4. Changes in the status of FCHs compared to non-familial care helpers 55

Table 4.1. A profile of interviewees who participated in the study 88

Table 4.2. Transcript notation $\quad 89$

Table 5.1 Positions in family care: traditionalists, negotiators and partners 177

\section{List of Figures}

Figure 2.1. Role of the insurer in the LTCI in Korea

Figure 2.2. Care agency in the service delivery in the LTCI 48

Figure 2.3. Process of assessing LTC recipients 49

Figure 2.4. The service delivery of home visit care after getting a licence $\quad 50$

Figure 4.1. The PCI topic guide $\quad 84$ 


\section{CHAPTER I INTRODUCTION}

\subsection{Introduction}

This study focuses on the experiences of family care helpers (FCHs) in the long-term care insurance (LTCI) for the elderly in South Korea (hereafter Korea). The population ageing and changes in family structures and the care culture since the 2000s led to the introduction of the LTCI for the elderly in 2008. Since the mid-1990s, there have also been some structural changes in the industry, in economic and political regimes and in women's participation in the labour market. These developments influenced the introduction of the LTCI, which was implemented in Korea on July 1, 2008, only nine years after it was initially discussed in 1999. The LTCI regulates items in elderly care services, which support physical activity or housework for the elderly who have difficulty taking care of themselves due to old age or geriatric diseases. The insurance aims to promote senior citizens' health and life stabilization, as well as improving the quality of people's lives by mitigating the burden of care on family members ( $\S 1$ of the Act on the LTCI for the elderly).

Elderly care is one of the core issues of the 'welfare mix' of the state, the market and the family, due to its specific characteristics, which are intangible and are based on relationships and interactions between the caregiver and the care receiver (Evers and Svetlik 1993l; Daly and Lewis 2000; Kwon Soo-Hyun 2013). In this sense, welfare states take measures to respond to deficiencies of elderly care, but the care policies that the state implements vary in the degree to which family members are engaged in these policies. The welfare states often attempt to subsume previous informal and family caregivers into the new formal elderly care systems that they are looking to implement (Colombo et al. 2010; Kodate and Timonen 2017). There are many ways of recognising the care offered by the informal and family caregivers, including care leave, pension credits and cash benefits such as care allowance or attendant allowance (Kremer 2007).

Making the informal formal by recognising family caregiving is one of the key issues in Western developed welfare states regarding the guarantee of inclusive citizenship and social rights to give and receive care (Knijn and Kremer 1997). However, there is no 
evidence to what extent the same principle can be applied to evolving welfare states, like that of Korea, which has been known for different welfare regimes and care cultures. The ways in which informal caregivers engage in the formal system differ from other countries that run very similar elderly care schemes, like Japan and Germany. In Germany, the elderly can choose cash payments and ask for their family members to be their caregivers in the sense of the consumer's choice (Pfau-Effinger et al. 2011). On the other hand, family caregivers in Japan are not allowed to be paid for caring for their elderly family members in any case, since the formalisation of the family caregivers would lead to women not entering the paid labour market as individual workers.

The Korean welfare state included informal family caregivers within the LTCI system. However, the government refused to offer direct payments to family caregivers, deciding instead to allow the family caregivers to become qualified caregivers by obtaining a care helper licence in order to receive cash for care. This study begins with an interest in the emerging tendency towards an increase in the number of family care helpers (gajokyoyangbohosa) who are licenced family caregivers under the long-term care insurance (LTCI) for the elderly in Korea. The term 'care helper' refers to a person who is qualified for elderly care in the LTCI. With regard to the qualifications and the contracts, there are no differences between family caregivers and care workers who care for elderly people with whom they are not in a family relation. When informal family caregivers want to care for family members as a FCH, they have to enter the same formal education and administration system like everyone else. For this reason, the FCHs can also offer LTCI services to elderly people in non-familial relations, as they have the same licence as nonfamilial care helpers. The FCHs' caregiving has not yet received full recognition. Even though family caregivers are qualified as FCHs and often offer in-home care for 24 hours every day, only one hour per day is recognised for the FCHs as formal caregiving. The rest of the hours of family care for the elderly are considered as family responsibilities. In other words, the government insists that the main responsibility of caring for the elderly lies with the family members and it is sufficient for the state to partly recognise or assist the family caregivers.

Given that they undergo the same process of qualification, the decision of the family caregivers to become FCHs is often considered as a mainly economical one, becoming care workers to enter the paid labour market. Indeed, there are studies suggesting that the 
implementation of the LTCI in Korea led to a defamilialisation of elderly care (Shin KyungAh 2011; Saraceno 2016). However, the FCHs are hidden in these statistics in that the LTCI services that the FCHs offer are counted as the gross domestic product (GDP) since the FCHs are paid for their family care as service providers of the LTCI programme. Previous studies show that women's participation in the paid labour market is a significant requirement for them to claim the same social rights as male workers in the labour market (Hobson 1990; Huber et al. 2009). Therefore, one can argue that the formalisation of care work allows care service users to reduce their own domestic care burden and to re-enter the labour market (Jung Youn and Kwon Soonman 2014; Seomun Jinhee and Jung Yeo-Joo 2011).

Nevertheless, I have some reservations about the argument that the family caregivers engage in the LTCI programme mainly in the sense of participation in the paid labour market. As was mentioned above, although the qualification process of the FCHs is the same as that of the non-familial care helpers, the FCHs' care is limited in recognition of care hours when compared to the non-familial care helpers' care. Whilst in-home care services in the LTCI offered by non-familial care helpers are recognised up to 240 minutes per day and 30 days per month at the maximum, the services offered by the FCHs are recognised only up to 60 minutes per day and 20 days per month. According to calculations based on payment standards for the family care helpers of the LTCI, a FCH is paid up to 170,000 KRW (about 170 USD) monthly ${ }^{1}$, which alone is not enough to maintain a livelihood and guarantee entitlement to pension and unemployment benefits. Given this limited recognition of the family caregivers, it is evident that the engagement of FCHs in the formal elderly care programmes should not be equated with entering the paid labour market. Thus, while FCHs may appear to be care 'workers', there is still a need for a more thorough investigation into the licenced family care experience.

There are several studies backing the findings that while the FCHs look as if they are entering the paid labour market, the payments they receive for their care work actually resemble those characteristics of care allowance for family caregivers (Lee Yun-Kyung 2010; Hong Sung-wook 2011; Seok Jae-Eun 2006). Since the LTCI in Korea did not accept

\footnotetext{
${ }^{1}$ When a FCH is a spouse of the recipient and is over 65 years old, payments for the care offered by the old spousal care helper are recognized by an exceptional standard of up to 90 minutes per day instead of 60 minutes and 30 days per month instead of 20. According to this standard, the spousal caregiver is paid up to $400,000 \mathrm{KRW}$ (about 400 USD) monthly.
} 
the care allowance, the family caregivers decided to become FCHs for the payments. The percentage of the FCHs rose significantly since the implementation of the LTCI (Yang Nan-Joo 2013) and most of them obtained a care helper licence for the sole reason of caring for one or more members of their family (Chang Kyung-Sup et al. 2015; Lee Jaerim 2015; Lee Min-Sook 2012). The payments for the FCHs remain contested. Ungerson (2000) suggests there are five different categories of financing elderly care: 1) caregiver allowances paid through the social security and tax systems 2) proper wages paid by the state or by state agencies 3) symbolic payments paid by care users to kin, neighbours and friends 4) paid volunteering paid by voluntary organisations and local authorities 5) routed wages paid via payments to care users. However, there has been little research into which category the payments for the FCHs' care for their frail family members should be placed in.

I assume that due to the very limited recognition of their caregiving, family caregivers' engagement in the LTCI means that they have other (or at least additional) reasons for becoming a licenced family caregiver. Since the relational nature of the care is embedded in elderly care, the family continues to play a pivotal role in it, allowing the formal and informal aspects of care to be mixed. As Colombo et al. (2011) argue, elderly care has been carried out by family caregivers (mostly women) at home for a long time and, despite the implementation of a formal long-term care programme, the rate of family caregiving remains high. Indeed, significant numbers of old people in Korea still continue to receive care from their family members after the introduction of the LTCI. According to the national health insurance co-operation (NHIC) in Korea (2012), 32.7\% of the total in-home care helpers in the LTCI were the recipients' family members. Studies focussing on why family caregiving has not decreased in Korea suggest the following reasons: 1 ) the elderly prefer family care, 2) family members are also more desirable in-home caregivers, and 3) from the financial perspective, informal caregiving mitigates fiscal constraints on public care provision (Kim Chulju and Hong Sung-Dae 2007: 238-43).

The engagement of former informal caregivers in the formal LTCI system would affect their citizenship regarding ‘social rights to give care’ (Knijn and Kremer 1997). However, given the context of the Korean welfare state, which can be categorized as a productivist welfare state (Wood and Gough 2006), several studies have claimed that the LTCI in Korea can be considered as not merely a social policy for the social rights of the elderly who are 
in need of care and their families but as an economic policy, which creates job opportunities in the social service economy (Kim Hee-Kang 2017; Lee Jaerim 2015; Seok Jae-Eun 2006).

Previous studies suggested several reasons why the Korean welfare state has encouraged family caregivers to become 'formal' caregivers. First, there was a need to expand the national care infrastructure, including care facilities, care educational institutions, management agencies (care centres), and trained care helpers (Choi Sung-Ho 2008). Until the 1990s, the traditional care culture, in which the eldest son's family was expected to care for their parents in requital for the inheritance of the family, was predominant in Korea. That is why the public provision of elderly care was underdeveloped, regardless of the level of family assets and income. Secondly, the government implemented the LTCI not only to mitigate the social risks caused by an ageing population but also to create new jobs in the social service sector (Oh Eunjin and Roh Eunyoung 2011). Thirdly, the government decided that a mixture of formal and informal care could create a complementary effect since the elderly tend to feel more comfortable receiving care from family members than from strangers. Indeed, bodily contact and assistance with everyday activities at home affect the elderly on both emotional and social levels (Kwon Soo-Hyun 2013: 5-16; Stacey 2011). Welfare states in the western countries also prefer in-home caregivers, since a family-centred care system can maintain 'the virtues of the family' and results in decreased government spending (Eichler and Pfau-Effinger 2009).

At the micro-level, family care for the elderly could be understood as a family practice. Like other East Asian countries, Korea has been known as a country where the familycentred care culture based on kin relationships is regarded as 'the proper thing to do' (Finch 1989; Finch and Mason 1991). Before the implementation of the LTCI in Korea, most care for the elderly had been provided by family members at home. This care culture is primarily grounded on the notion of 'hyo (효, 孝)', which means filial piety in Korean. Until the 1990s, in Korea, the eldest son was the one who was symbolically responsible for caring for the elderly, as he had the duty of caring for his parents and performing ancestral rites in exchange for inheriting his parents’ property. However, in practice, the care responsibilities are usually not carried out by the eldest son himself but rather by his wife. This is owed to the gendered division of domestic versus gainful work - care work falling into the category of domestic work and therefore considered the responsibility of the wife who will care for her parents-in-law. In this sense, caring for the elderly is also considered not as a form of 
labour but as an attitude. Studies on the motivations of becoming a FCH show that often the family caregivers decided to become a FCH due to a sense of family obligation in the context of the traditional care culture in Korea (Yang Nan-Joo 2013; Lee Minsuk 2013; Choi Inhee and Yanng Nan-Joo 2014).

Recent studies on professional care for the elderly have suggested that staying at home with family care until the end has been the ideal of elderly care in Korea, because of the traditional care culture and because of the underdeveloped care infrastructure (Kim Jiyeon and Kim Hongsoo 2016). However, as Finch (1989) points out, normative guidelines within a society are likely to be general and judgemental rather than detailed and specifying particular actions. In this sense, 'hyo (효, 孝)' as a cultural norm is also changeable in terms of how the family deals with their family issues in reality. The first son's obligations have been subject to very little empirical investigation in the years since the implementation of the LTCI.

Meanwhile, the care provided by FCHs can be explained in the context of family practice in the sense that the ways and settings of elderly care in Korea are decided by more family members than just the caregiver who actually cares for the elderly. Previous studies on informal family care reported that a family's actions in daily life are based on their sense of responsibility for elderly care (Cho et al. 2015; Lee Min-Sook 2012; Seo In-soon 2003; Yang Yeung Ja 2018). Family members who have been determined to care for their old family members, which (for the reasons mentioned above) are mainly women such as daughters(-in-law) and wives, often experience pronounced care burdens but continue to provide elderly care at home because of societal and familial expectations. These care burdens can lead to negative forms of stress such as anger, guilt and frustration. On the other hand, the caregivers can also experience positive feelings in caring for their elderly family members, like, for example, being proud of caring for the elderly or an improvement in their relationship with the elderly and/or with other family members.

However, the traditional care culture began to change when Korean society began to industrialise which lead to things like an increase in economic participation of women and a decrease of the classical cohabitation model of the three generational families. Under these newly emerging circumstances, the responsibility for care has been increasingly moved from the eldest son (and/or his wife) to other family members, such as younger sons, daughters and spouses. In Korea, the decision of a family caregiver to become a FCH can 
be assumed to have various reasons, such as pursuing economic incentives, a way of gaining recognition of family caregiving, positive interactions in the sense of family practices, or imposed obligations based on the social and familial expectations. Yang NanJoo (2013) also formulates the following reasons for informal family caregivers to obtain a care licence: the maintenance of their own livelihood and caring at the same time, because of the children's obligation to their parents and because of shortages of available services under the existing ways of caring (Yang Nan-Joo 2013).

After the implementation of the LTCI for the elderly in Korea in 2008, significant numbers of informal caregivers became a FCH (gajokyoyangbohosa), who is a qualified and paid family caregiver in the LTCI. The Korean welfare state's way of making the informal formal differs from the Western developed welfare states, whose care policies have been derived from the individual's social rights to give (and to receive) care. Prior research has extensively investigated how the European welfare states share care responsibilities among actors in society. However, little research has been conducted to investigate how these responsibilities are distributed among actors in East Asian countries that are characterized by different societal, institutional and cultural backgrounds.

In Korea, there have been studies on family care for the elderly, but these have focused on the conflicts among family members in caring for the elderly in informal settings (Kim Tae-Hyun and Jun Gil Yang 1995; Kim Hyunjoo 2001; Seo In-Soon 2003). Although the FCHs are a significant element that shows the family-centred care culture in Korea, there has been little research on them specifically. After the implementation of the LTCI, most research has been focused on the effects of family caregiving on fiscal sustainability (Lee Yun-Kyung 2010; Hong Sung-Wook 2011). In order to gain a comprehensive understanding of the care culture, however, it is vital to investigate the lived experience of the FCHs, since the same care policy can lead to a plurality of consequences for the daily life of different actors, due to the different social contexts in which the individual experiences these policies.

\subsection{Aims, Research Questions}

The central aim of this study is to explore how the family caregivers experience their activity as FCHs (gajokyoyangbohosa) who are qualified within the LTCI programme. Specifically, this study investigates the family caregivers' perceptions on their social status 
regarding social rights to provide care (Knijn and Kremer 1997) and their response to expectations on the care responsibility for their old family members.

Since this study is mainly concerned with the effects that public care provisions have had on the experiences of FCHs, it focuses on the period since 2008 when the LTCI was introduced. Furthermore, the study investigates care workers who started caring for their old family members before the introduction of the LTCI, as this will facilitate exploring the changes it brought to family caregiving. In order to understand the experiences of FCHs in both the public and domestic domains, FCHs who provide only in-home care services for their old family members are selected for this study; the experiences of FCHs working in care facilities are beyond the scope of this study. This investigation was not limited to nuclear family relations, but aimed to include spouses, children, in-laws, cousins and other distant relatives, as previous studies have shown that family members can influence the motivation and patterns of caregiving (Han and Son 2009; Pinquart and Sörensen 2011; Tennstedt et al. 1993). Although some studies insist that there are no significant effects relating to the type of carer (Kim Ju-Sung et al. 2004; Lee In-Jeong 2006), spouses are likely to experience more symptoms of depression and greater financial and physical burdens, since they have different socio-demographic conditions compared to children and children-in-law (Pinquart and Sörensen 2011: 8). Especially in Korea, daughters-in-law were primary carers, due to the complex of gender norms and filial piety based on the Confucian care culture, but this responsibility has passed to the spouses of the elderly, leading to confusion among spouses (Han Gyoung-Hae and Son Jung-Yeon 2009). In this context, the study explores the diverse experiences concerning family relations in caring.

It is anticipated, that through a better understanding of the FCHs' experiences on becoming licenced caregivers and the issues and challenges they face in daily life, more wellinformed decisions on settings of elderly care can be made by both families and policymakers. To shed light on the phenomenon mentioned above, the following three research questions are addressed:

\section{Q1: What is the meaning of caregiving as a FCH?}

This might depend on how FCHs experience changes in their daily life after becoming a FCH in the LTCI. Through an investigation of the process of making meaning on their own, 
this study attempts to understand the meaning of becoming and being a FCH from the perspective of the FCHs themselves.

\section{Q2: How do the FCHs construct their positions as users and service providers of the LTCI?}

The FCHs are one of the actors in sharing the care responsibility for the elderly in Korean society in that they are both users and service providers in the service delivery system in the LTCI. This question could be divided into these particular questions: What are the meaning of their caregiving and the payments for care? Do they consider themselves workers or family members? Why do they think in this way? Are they satisfied with their social status after becoming a FCH? If they have some problems in the course of caregiving, how do they cope with that?

\section{Q3: How do the FCHs take the care responsibility for their old family members?}

As family practice, the FCHs' caregiving might be a result of the interactions among family members. A decision on who cares for the old family member(s) might depend on the care culture, on particular conditions and contexts of the family the FCHs belong to.

\subsection{Approach, Assumptions and the Significance of the Study}

To investigate the experiences of the FCHs, I apply a qualitative empirical research approach, which is designed to access family care helpers' (FCHs') experiences in everyday life, as well as to generate new theories (Reinharz and Davidman 1992). Specifically, I focus on the life-course of the FCHs, since family care occupies long periods of one's life. In addition, the history and relationships within the family would have an impact on the experiences of the FCHs in caring. Thus, considering the life-course of the research subjects will enable us to investigate the meaning of being a FCH in greater depth.

Based on the background assumptions and the research questions mentioned above, I set out my initial assumptions as follows:

Firstly, the social status of the FCHs would be situated somewhere between workers and family members. Although they gave care to the elderly before, they have experienced differences in the care before and after entering the LTCI system. First, to care for their old family members as a FCH, an informal caregiver has to be qualified by licencing for a care 
helper and to register in the LTCI programme. They are under the supervision of the LTCI programme, which manages their caregiving in order to calculate the payments for their care work. Although they work at home and have impartial entitlements to social insurance because of their status as a FCH, their caregiving would be specifically organised and recorded by the LTCI centre, like that of any other care helpers. In addition, the FCHs are likely to experience conflicts with other family members in different contexts. This study investigates FCHs' experiences in caring for the elderly and examines how they are linked with their status.

Secondly, through the introduction of the LTCI programme, the FCHs would be concerned about their social rights as individual workers - for example, their entitlement to the national pension system (NPS), to unemployment insurance and to occupational insurance. At the same time, the FCHs would feel that this is unfair in that they have to take on all the tasks of care for the elderly since they are 'licenced and paid' caregivers from the LTCI programme.

Since various factors in individual life history, such as education, work experience and career, family status, level of income, can feature into the decision of a family caregiver to become a FCH, the paths to becoming a FCH might differ considerably. Through exploring the care and work histories of individual care workers in detail, this study aims to suggest various factors, which can be relevant in becoming a FCH.

In sum, I expect that the family care helpers (FCHs) will change their perceptions of status and identity from that of a family caregiver to that of a wage worker, and this will have made them confused in that they would not be supported either by social insurance benefits in the public sector by the state nor for the tasks of elderly care by other family members. Against this background, I mainly focused on the changes in FCHs' social status as both wage workers and family members at the same time.

An investigation of the experience of the FCHs will reveal how the responsibility for elderly care is shared on two different levels. At a macro-level, the experience of the FCHs shows how the state, the market and the family share the responsibility for elderly care in the sense of 'social care' and a 'responsibility mix' among actors in social care. Specifically, understanding how the semi-formal caregivers perceive the social right to give care will help us to orient elderly care policies in Korea given the findings of this study. 
Although the regulations on payments for family care have been changed several times, there were no proper standards and justifications for the regulation revisions. At the microlevel, the lived experience of the FCHs could provide an overview of the process of becoming a FCH, which will enable us to see how positive and negative factors in the care for elderly family members are interwoven throughout.

\subsection{Outline of the Chapters}

The thesis consists of seven chapters, including the introduction and the conclusion. Following this introductory chapter, Chapter II will provide the context for life in old age, family relationships and the LTCI in Korea. The traditional forms of intergenerational financial supports, cohabitations with adult children and personal care in Korea have been changing in a way that has led to their decrease. This chapter will also describe the research tendencies on elderly care and family caregivers since the 1990s. Furthermore, the LTCI will be explained in more detail, in terms of the subject, provisions, financing and the services it provides. Here I also describe how responsibilities for elderly care are shared between the government and the family in Korea by analysing the trajectories of recognition hours for LTCI services offered by FCHs.

In Chapter III, I will review the literature on how elderly care is provided among different actors in society at both the macro- and the micro-level. The literature on care responsibility, social rights, and social care is expected to provide the theoretical grounding for this research. In this chapter, I will also present an analytical framework to interpret the experiences of the family care helpers in Korea. Based on the literature review and the analytic framework, Chapter III will conceptualise care responsibility at a macro- and a micro-level. Gaps in the previous literature will be identified, including the lack of any studies of the lived experience of the FCHs who have a status somewhere between workers and family members.

Chapter IV explains the research methods, which I have applied in my empirical study. This chapter will provide a methodological framework, which is based on an understanding of social reality 'as the result of meaning and context that is jointly created in social interaction' (Flick et al. 2004: 6). The research process will be explained, including data collection, data analysis and ethical considerations. I adopted the problem-centred interview (PCI, Witzel and Reiter 2012) as a method of a data collection. I present a brief 
discussion of the PCI interview, which combines narrative and semi-structured interview strategies. This chapter will continue with a description of the steps that the PCI proposes for data collection, sampling strategies and interview flow. As a strategy of analysis, I have adopted an empirical analysis of biographical narrative interviews. I interpreted the experiences of the interviewees according to biography-oriented analysis and established three case reconstructions according to the suggestions of Rosenthal (1995; 2011; 2018).

In Chapter V, an in-depth analysis through the interpretation of the interview texts with the family care helpers will be undertaken to investigate the experiences of the family caregivers who care for their family with a care helper licence. Firstly, I pick up three cases and suggest my interpretations of the FCHs' experiences that are oriented along the presentation of the biographical case reconstructions. The empirical evidence will be compared to my initial assumptions, and I offer a provisional result regarding the caregivers' perceptions of their social rights as a FCH and the meaning of care for their old family members. This chapter also provides the three types of FCHs that I worked out as a result of my analysis and summarises the structural characteristics representative of each type. The investigation into the experiences of the FCHs will provide two different approaches at the macro- and micro-levels. At a macro-level, this chapter shows how the FCHs' perception of their social rights after the implementation of the LTCI and how the FCHs perceive their experiences regarding family relationships at a micro-level.

The final chapter will revisit the main findings and discuss the implications of the study, as well as make suggestions for future research. It offers a summary of the previous discussions in the light of the analytical framework, research questions and empirical findings. The final chapter also includes reflections on the relevance and value of the concept of care responsibility for Korean society. The chapter ends with some concluding remarks on the experiences of the FCHs, the limitations of this study and further research suggestions. 


\section{CHAPTER II LATER LIFE AND FAMILY RELATIONS IN KOREA}

\subsection{Introduction}

This chapter explores the current living status of the later life and family relationships in Korea where ageing of the population has been rapidly increasing since the 2000s. Based on an understanding of later life and family relations in Korea, the difficulties and needs of the present elderly as well as of other family members can be revealed.

Before starting this chapter, the definition of "old age" and "the elderly" as used in this study is needed. According to the Oxford English Dictionary (2018), the term "old age" comprises "the later part of life; the state of being old", but the scope of the elderly differs according to the context. The United Nations (2017) defines the elderly as people aged 65 years and more, and most institutions and Administrative Law in Korea set the age of 65 for retirement. For example, to be 65 years old is a basic requirement for becoming eligible for old-age pension benefits of the National Pension System (NPS) in Korea. However, the onset of old age could be set anywhere from the mid-fifties to the seventies given the fact that the definitions of old age have been continuously changing; especially as life expectancy in Korea has risen to beyond 80 years (KOSTAT 2017b). Indeed, the life expectancy of people who were born in Korea in 2016 averages 82.36 years. Specifically, women have a longer average life expectancy (85.41 years) than men, who have that of 79.30 years. Besides, in a survey on the living standards of the elderly and welfare need in 2014, most participants answered that the elderly are denoted those who are 75 and older (Jung et al. 2014). On the other hand, the expected retirement age in Korea has decreased from 60 years in 2000 to 57 years in 2016. Against this backdrop, the Employment Promotion for the Aged Act in Korea set "the aged" as those who are 55 years old and older. In this sense, I use the scope of old age and the elderly flexible from 55 to 85 years and more and put focus rather on identifying the trends of the aged and the ageing in Korea.

The number and the proportion of the elderly in Korea have continued to increase for decades. In 2017 the number of the elderly aged 65 and older amounts 7,076 thousand persons (KOSTAT 2017b). The overall population in Korea is younger than in most of the developed western countries, the speed of the ageing in Korea, however, recorded the fastest among the Organisation for Economic Co-operation and Development (OECD) 
member countries (OECD 2017). Korea entered the 'ageing society' as people aged 65 and older composed $7.4 \%$ of the Korean population in 2000, but the proportion of the elderly has rapidly increased to $13.8 \%$ in 2017 , which means it took only 17 years to transfer from the 'ageing society' to almost reaching parameters for the 'aged society'. This is the combined effect of an increase in life expectancy and a decrease in the already low birth rate. KOSTAT (2017a) forecasts that the number of working-age population, who are aged between 16 and 64, will start to decrease from 2017 onwards, as the Korean baby-boomers, who were born between 1955 and 1963, start to retire from their jobs. Given the changes in the demographic structure in Korea, the life and trends of the elderly could change in many aspects.

While the proportion of the elderly in Korea has been continually increasing, most of the elderly in Korea live in low standards. Studies on the elderly in Korea point out the harsh living conditions of the later life in Korea by referring to "Four Pains in Old-Age in Korea", including poverty, diseases, idleness and solitude (Park Keong-Suk and Kim Mi-Seon 2016; Kim Dong-Bae et al. 2012; Lee Ju-Eun and Park Myung-Suk 2012; Song YoungHeum 2012; Nah Hang-Jin 2003). Notably, the relative poverty rate (proportion of people below 50\% of median income) of people aged 65 and above in Korea was 49.6\% in 2016, which is the highest rate compared to other OECD member countries (OECD 2017). In addition, Chang Ji-Yeon et al. (2008), who conducted a comparative analysis on the midaged workers of the labour markets in developed countries and Korea, argued that the elderly in Korea have four peculiarities compared to the elderly in developed countries: 1) a high poverty rate 2) high level of income inequality among the elderly compared to developed countries 3) a steeper decline of income as one gets older, and 4) a low proportion of public transfer and high proportion of labour income in old age.

Against this backdrop, this chapter illustrates the living status of later life and family relations in Korea. Section 2.2 explains the later life in Korea by categorising several elements: old age income security, housing, health care and social services. Specifically, section 2.2.1 describes how the elderly in Korea make a living with three different types of income sources, including public and private income transfer as well as labour income. Section 2.2.2 explains housing in later life as both perspectives of the property and space for living. Cohabitation of the old parents and the first son's family as Korean old living tradition has been diminishing. In section 2.2.3, the elderly's increasing needs for health 
care and social services will be described. After reviewing the elderly's status in Korea on an individual level, I explore the elderly's life on the interpersonal level by describing trends and changes in family relations in Korea in section 2.3. Recently, Patriarchal familism and the first son centred care culture has been diminishing, and new types of caregiving trends have started to emerge. I start by reviewing the concept of 'hyo (효, 孝)', which is a traditional moral value in Korean familism. Then, I explain how the traditional care culture in Korea has changed in recent years. An overview of research trends in elderly care practice among families in Korea will also be given in section 2.3.2. Based on the review in section 2.2 and 2.3, I examine the position of family care helpers (FCHs) of the LTCI in Korea by describing the service delivery of the LTCI. I assume that the introduction of the FCH programme is a result of a combination of factors, namely the underdevelopment of welfare programmes and the family-centred care culture in Korea. Therefore, section 2.4 focuses on the status of FCHs by tracing changes in the regulations of the LTCI on family caregiving from its introduction to the present. The chapter ends with a conclusion, suggesting the implication of the status of the FCHs on the LTCI.

\subsection{Living Status in Later Life}

This section examines the living status in later life in Korea. I first describe means of oldage income securities, which comprise public income transfer, private income transfer, and labour income. Then I move onto the housing status, which shows that the traditional cohabitation with adult children has decreased as a result of changes in traditional family culture. The last section 2.2.3 gives information on the health care scheme for the elderly in Korea.

\subsubsection{Old-age income security in Korea}

According to the 2013 Household Income and Expenditure Survey, about 35\% of the total elderly households2 were beneath the absolute poverty line and whose income was below the minimum cost of living, and about $56 \%$ of the total elderly households lived in a condition of relative poverty (Lee Seong-Lim 2015: 87). Although there are public transfers for the elderly, like public pensions and public assistance, the elderly in Korea mainly rely on private support from their adult children and working wages in their later life. Indeed,

\footnotetext{
${ }^{2}$ In the survey, an elderly household was defined as elderly singles or couples with both spouses aged 60 years or more without any other household members (Lee Seong-Lim 2015: 111).
} 
the poverty reduction effect of the national pension benefits was only $4.9 \%$ p for a single elderly and $11.1 \%$ p for a couple elderly household, and the reduction effects were overall relatively low compared to other OECD members (Yeo Eu-Gene 2013: 185).

Incomes in later life are categorised into three types: public transfer, private transfer, and labour income. First, a public transfer includes public pension benefits based on contributions of the insurance, a tax-based allowance for the elderly, and tax-based and means-tested public assistance. Second, a private transfer includes economic supports from family members and relatives in the sense of inter-generational supports or savings from one's working ages. Last, the labour income of the elderly refers to an income source generated by the elderly's present work and labour.

\section{Public income transfer}

The Korean public transfer scheme can be seen as a multi-pillar system, in which one gets their retirement income from various sources. In addition, each income security programme has different introduction timing and contexts. For example, the first introduced income security programme for later life was a pension for public officials in 1960, whose recipients are relatively stable in livelihood compared to other workers. In contrast, the National Pension Service (NPS) for general employees was introduced in 1988, and the Basic old-age pension was introduced in 2008. A chronicle of introductions of income security programmes in Korea looks as follows:

1960: an introduction of a pension for public officials

1963: implementation of a pension for public officials and a military pension

1975: implementation of a pension for private school teachers and staff

1988: implementation of the national pension system (NPS) ${ }^{3}$

1999: the first old pension benefit was paid from the NPS

2000: implementation of the national basic living security (NBLS)

2007: nominal income replacement reform of the NPS from $60 \%$ to $40 \%$

2008: implementation of the basic old-age pension

2014: increase in the basic pensions benefit from 94,600 KRW to 200,000 KRW

\footnotetext{
${ }^{3}$ The eligibility of the NPS was only for employees who work in a company hiring more than 10 employees in January 1988. Afterwards, the eligibility has widened towards all employees working in a company hiring more than 5 employees as a statutory insured in 1992 as well as the self-employed and economically inactive population as a voluntarily insured in 1999.
} 
I discuss three principal old-age income security programmes regarding their eligibility and financing: the national pension system (NPS), the basic old-age pensions, and the national basic living security (NBLS). The NBLS is public assistance, thus, is not exclusively for the elderly. Yet, I explain the public assistance because the proportion of the elderly over 65 among the recipients of the NBLS is very high and therefore the programme plays a significant role as an income source in later life in Korea.

The NPS is a major public pension in Korea in which all economically active and inactive population, except persons insured under the special occupation retirement pension, are entitled to insurance coverage. Although a discussion on the introduction of a public pension system for employees had emerged in the early1970s, the introduction of the NPS was postponed because of the oil shock in 1973, so that the actual implementation of the NPS did take place in 1988. The NPS is contribution-based insurance in principle, but the government shares only a part of administration cost as well as subsidies for contributions of farmers.

In addition to the NPS, there are three other public pensions for those in special occupations, including government employees, professional soldiers, and private school teachers and staffs. These three special occupational pension plans were introduced much earlier compared to the NPS. A pension for public officials and a military pension were implemented in 1963, later a pension for private school teachers and the staff was implemented in 1973.

Old-age pension benefits of the NPS are calculated according to factors of not only years and level of contributions of individuals entitled but also the average income of contributors entitled. The entitlement of old pension benefits requires at least 10 years of contributions in one's working age. In this sense, the benefits system of the NPS has a characteristic of redistribution between contributors but is likely to neglect income security of the present elderly who did not have enough opportunities to fulfil the minimum requirement of the old pension benefits.

In addition, the coverage and the absolute level of old pension benefits of the NPS are low. According to the survey on the living standards of the elderly and welfare need in 2014, the number of the elderly aged 65 years older was 6,521,000 persons in 2014, and only 
2,569,000 persons (39.4\%) among them received public pension benefits (Hwang NamHee 2016: 26). The average of monthly benefits amounts 277,000 KRW (about 277 USD). Moreover, the rate of excluded and uninsured persons relating to the NPS is relatively high, and most of them are the small-scale self-employed, low-paid irregular employees, the unemployed, and housewives, who are more likely to be driven into poverty in later life (Yeo Eu-Gene 2013:213).

As the NPS is predominantly run on a funded by contribution basis, the issue of management of the fund has emerged. Specifically, the Ministry of Health and Welfare (2007) estimated that pension funds would begin shrinking in 2020 due to inflation and the low birth rate and ageing population in Korea. Therefore, after debates on pension reform, the government and the assembly agreed on a progressive reduction in nominal income replacement of the NPS from $60 \%$ in 2007 to $40 \%$ in 2028. To offset decreased income replacement of the NPS and to alleviate poverty in later life, the government introduced basic the old-age pension which is a tax-based allowance. According to a survey on the living standards of the elderly and welfare need in 2014, 92.9\% of the respondents had public transfer incomes, while only $31.9 \%$ of the respondents received contribution-based pension benefits (Jung et al. 2014).

As mentioned previously, the basic old-age pension was introduced against the background that the present elderly could not have the opportunity to be eligible to the NPS old benefit as the NPS was implemented in 1988 and on a funded basis. As mentioned before, only $39.4 \%$ of the present elderly receive old pension benefits under the contribution-based pension scheme, including the NPS, special occupational pension schemes like the pension for public officials, the military pension, and the pension for private school teachers and staff. Those who have no individual pension benefits had been likely to rely on support from their adult children in the sense of the first son's obligation as a traditional Korean care culture, but the support from children has been decreasing due to changes in family structure and care culture for the elderly. As a result, the poverty rate in old age in Korea reached 49.6\% in 2012 which was the highest rate among OECD member countries (OECD 2015b). Therefore, there was a need for new public income aids to offset the deficit of oldage income. 
Against this background, the Roh Moo-Hyun government planed a basic old-age pension as a new counteragent to the poverty of the present elderly in Korea ${ }^{4}$. Moreover, the introduction of a new flat-rate and tax-based old-age income support programme for the elderly intertwined with the NPS which was in a debate on pension reform in 2007. After a lively controversy, the basic old-age pension was implemented in 2008. It requires no contribution but is tax-funded. Although the basic old-age pension is provided on a meanstested basis, the means-test is relatively generous and $60 \%$ of the elderly who are 65 and older could receive the flat-rate benefits with 94,600 KRW (about 90USD) per person monthly.

Several studies noted that the basic old-age pension contributed more significantly to reducing the poverty in old age than the NPS since the coverage of the basic old-age pension is much wider than that of the NPS (Yeo, Eu-Gene 2013). Unlike the NBLS benefits, the basic pension benefits do not take into consideration the economic capability of the elderly's adult children. The eligibility to the basic pension depends on the elderly's economic status as an individual. Indeed, the basic old-age pension benefits became one of the national concerns in the presidential election of 2012, and one of the presidential candidates, Park Geun-Hye, had pledged to increase the coverage and the level of the benefits of the basic old-age pension. As a result of his election, the Park Geun-Hye government extended the coverage from $60 \%$ to $70 \%$ and increased the level of benefits from $94,600 \mathrm{KRW}$ to $200,000 \mathrm{KRW}$ in 2014. In addition, the name of the "basic old-age pension" was changed into the "basic pensions" in terms of underlining universality of the concept.

In addition to the public pensions and the basic pensions, the national basic living security (NBLS) plays a pivotal role in public income transfer system in old age in Korea. In 2016,

\footnotetext{
${ }^{4}$ Article 1 of the basic old-age act in 2008 declared the purpose of the basic old-age pension as follows:

"The purpose of this Act is to support the stabilization of the livelihood of low-income senior citizens and promote their welfare by providing them with basic old-age pensions in consideration of the senior citizens, contribution to bringing up their descendants and advancing the country and society." The act concerned the present elderly's contributions to society and focused on the elderly with low incomes. Later, there emerged debates on the level of the pension benefits and the coverage in the presidential election in 2012. In result of debates, the level and the coverage of the basic pensions were increased, and in this course, the name and the purpose of the basic old-age pension have changed in 2014 into the basic pensions. In addition, the purpose of the basic pensions focuses on income security of the elderly in general instead of the livelihood of the elderly with low incomes, and the pension benefit for the elderly is provided in terms of the citizen's rights. The amended version of Article 1 of the basic pensions act since 2014 reads as follows: "The purpose of this Act is to support the stabilization of the living of senior citizens and to promote their welfare by providing a stable income base through payment of basic pensions.”
} 
the number of beneficiaries of the national basic livelihood with 65 years and over was about 421 thousand, which accounts for $27.3 \%$ of the total NBLS beneficiary of 1,540 thousand persons (KOSTAT 2017b). Researcher insists that public income support programmes in Korea do not work properly, neither individually nor as a collective of all measures (Lee Seong-Lim 2015; Yeo Eu-Gene 2013).

\section{Private income transfer}

Even if the share of public transfer among old-age income sources has been rising in Korea, private income transfers still remain the most common income source in Korea. Traditionally, the elderly had been supported by family members, mainly the first son's family, and they had lived together with the first son's family in the sense of a Korean traditional patriarchal family system. By a cohabitation with and being supported by their adult children, the elderly in Korea tried to mitigate their poverty. However, the cohabitation rate of the elderly and their adult children has been decreasing since the 1990s and the poverty reduction effects have also weakened (Hwang Nam-Hee 2016: 32).

Nevertheless, children's financial support for their parents is relatively high compared to western welfare states, and this trend can be understood as family's responses to the present elderly's difficulties regarding financially independent living on public pensions, that were first introduced in the late 1980s as part of the NPS (Yeo Eu-Gene 2013). A lot of Korea's elderly get their main support from their family members, mainly adult children. According to a national survey on the household income and expenditure in 2013 by Statistic Korea, the percentage of the private transfer in the total income of the elderly households accounted for an average of $49 \%$ in case of single elderly households and for $25.9 \%$ in case of couple households (KOSTAT 2013).

\section{Labour income}

When one has not enough preparations for old-age income by in the public or private transfers, the elderly have no options but continuing working in old age. Compared to other OECD countries, the elderly in Korea work more hours. An employment-to-population rate of the elderly with 75 and over in Korea has been the highest level among OECD country members since 2013, and that of the elderly with 65 and over ranked number two after Iceland in 2016 (OECD 2017). 
According to the Senior Statistics by KOSTAT (2017b), 62.4\% of respondents between 55 and 79 years old wanted to work, and the percentage of the elderly who are willing to work in their late life has been increasing over the last three years. There are many reasons given by the elderly for working in their late life. The elderly in Korea work mainly to cover their living expenses since they have not prepared enough income source from the public and private transfers. According to NPS (2016), the respondents of the survey of the later life in Korea answered that the elderly in Korea need 1.6 million KRW (about 1,600 USD) for a couple, and 990 thousand (about 990 USD) for a single month as a minimum income for living their later life, and they need 2.55 million KRW (about 2,550 USD) for a couple, and 1.42 million KRW (about 1,420 USD) for a single monthly income to live a quality life. In any case, it is hard to cover living costs on public income transfer alone in Korea.

Moreover, when the elderly cannot obtain sufficient financial support from their family, they have to work for their living in old age. Therefore, the government introduced the Senior Job Supporting Programme in 2004. The Job Supporting Programme aims to create jobs for the elderly who are willing to earn incomes and to create an opportunity for them to participate in society (Song Young-Heum 2012: 180).

As mentioned before, there are various sources of income for late life in Korea, though they are still heavily weighted in favour of private transfers, as individuals and family take responsibility for financially supporting the elderly, rather than the state and/or society. Table 2.1 gives a brief description of the structure of income sources in later life.

Table 2.1. The structure of income sources schemes in later life in Korea

\begin{tabular}{l|l}
\hline \multicolumn{1}{c|}{ Type of income sources } & \multicolumn{1}{c}{ Programmes } \\
\hline \multirow{4}{*}{ Public transfers } & $\begin{array}{l}\text { The National Pension System (NPS) } \\
: \text { contribution-based old-age pension since 1988 }\end{array}$ \\
\cline { 2 - 2 } & $\begin{array}{l}\text { The basic old-age pension (the basic pension after } \\
2014) \\
: \text { tax-based old-age pension since } 2008\end{array}$ \\
\cline { 2 - 2 } & $\begin{array}{l}\text { The National Basic Livelihood System } \\
\text { : means-tested public assistance since 2000 }\end{array}$ \\
\hline \multirow{2}{*}{ Private transfers } & Private pensions \\
\cline { 2 - 2 } & Financial support from family \\
\hline \multirow{5}{*}{ Labour income } & Self-employed (e.g. farmer, small business owner) \\
\cline { 2 - 2 } & Employed in the private sector \\
\cline { 2 - 2 } & Employed in a public sector (e.g. the Senior Job \\
& Supporting Programme) \\
\hline
\end{tabular}

Source: Author 


\subsubsection{Housing in later life in Korea}

Residence stability is one of the most important requirements for quality life in old age. As residence stability plays a pivotal role in human dignity and value, housing conditions have an impact on quality of life and social relations. To give an overview of housing circumstances in old age in Korea, I describe the housing status of the elderly from two different perspectives. One perspective is housing in the sense of residence stability and property. The other is housing in terms of changes in family structure and cohabitation with adult children.

Housing problems in Korea have begun due to various rapid developments after the Korean War in 1950. After the Korean War, there were massive migration movements and drastic economic developments in Korean society. Due to urbanisation since the 1960s, significant numbers of people in Korea had moved from more rural areas to big cities to get a job in an industry sector. This rapid rural-urban migration and an urban concentration led to a shortage of housing. This tendency has continued until now, and the housing policy still remains to be one of the major issues in political elections and debates. Against these backgrounds, a house functions as one of the most important properties for a stable life in Korea. According to a survey on the households' finances and welfare, the proportion of real estate in total households' assets amounts 69.8\% in 2017 (KOSTAT 2018).

Since the significant need for stable housing has remained one of the most important contemporary issues in Korea, the housing policy is a topic of public and political debate. However, the housing policy has been mainly s focused on ownership of a house, while the share of public rental housing provisions has been relatively low. The reason being that in many cases, a house is the only property for households in Korea, and house owners have expected the value of their house to rise. It is common in Korea that one buys a house with a mortgage loan, and the house is leased out to a tenant for paying the mortgage.

Meanwhile, a house means not only a physical place to live but also space where one interacts with others. Not only ownership of a house but also the question with whom one is living with is important in the sense of residence stability, and the cohabitation with adult children affects the elderly's quality of life and well-being (Park Kyung-Suk and Kim MiSeon 2016; Kim Dong-Bae et al. 2012). 
During the later stages of their life, the elderly usually experience a decrease in the number of members living with them from marrying off their children and spouse's death as well as a reduction of economic activity after retirement. In addition, a deterioration in the elderly's condition can also have an impact on housing formation. Indeed, there is a tendency emerging for families deciding to live together when the elderly get frail and need medical treatment and persons who care for them - even if the elderly lived separated from the adult children before.

The elderly household structure has also changed towards an increase in single elderly households and a couple of elderly households. According to the future household projection by KOSTAT (2017a), the number of the elderly households, that have at least one family member with 65 and over, is about 3,999 thousand which accounts for 20.5\% of the total households in Korea. Moreover, the proportion of elderly households continues to increase and will be forecasted to reach $47.7 \%$ in 2045 (see Table 2.2).

Table 2.2. The trends of elderly households

(Unit: 1000 households, \%)

\begin{tabular}{c|c|c|c}
\hline \multirow{2}{*}{} & \multirow{2}{*}{ Total households } & The elderly households & Proportion \\
\cline { 3 - 4 } & 14,507 & 1,734 & 11.9 \\
\hline 2000 & 16,039 & 2,350 & 14.7 \\
\hline 2005 & 17,495 & 2,923 & 20.5 \\
\hline 2010 & 19,524 & 3,999 & 22.8 \\
\hline 2017 & 20,174 & 4,607 & 28.3 \\
\hline $2020^{\text {a) }}$ & 21,014 & 5,944 & 33.9 \\
\hline $2025^{\text {a) }}$ & 21,641 & 7,336 & 39.2 \\
\hline $2030^{\text {a) }}$ & 22,067 & 8,656 & 44.3 \\
\hline $2035^{\text {a) }}$ & 22,306 & 9,890 & 47.7 \\
\hline $2040^{\text {a) }}$ & 22,318 & 10,653 & \multicolumn{2}{c}{} \\
\hline $2045^{\text {a) }}$ & & & \multicolumn{2}{c}{} \\
\hline
\end{tabular}

Note: a) an estimated number

Source: Populations and Housing Census, Statistics Korea 2017a.

Many of the old people living without their adult children used to live in rural areas due to rapid rural-urban migration of their adult children caused by industrialisation. However, the proportion of the single elderly households and the couple household in urban areas has increased due to the ageing and retirement of the Korean baby-boomers who were born between 1955 and 1963 and have lived in cities and worked in industry sectors. 


\subsubsection{Health status and social services}

As one gets old, one's appearance changes and physical functions continue to decline. The elderly are likely to be vulnerable to chronic diseases like hardening of the arteries, high blood pressure, diabetes, osteoporosis, and dementia. Moreover, a high number of the elderly suffer from two or more diseases at the same time, and the complex senile disorders are hard to cure, while the symptoms get worse. The health status of the elderly has a significant impact on their quality of life in that a weakened health status leads to various difficulties in daily life and mobility that at some point make it hard for the elderly to live an independent life. According to the survey on living profiles and welfare service needs of older persons in Korea in 2012, 88.5\% of the respondents with 65 and over suffered from a chronic disease, and $68.3 \%$ of the respondents had more than two chronic diseases. In addition, the percentage of the elderly who took medicine for more than three months were $84.0 \%$ of total respondents of the survey (Jung Kyung-Hee et al. 2012).

Especially, the oldest persons of the spectrum with 85 and over are more likely to be exposed to health problems like chronic diseases. As life expectancy in Korea has increased over the last decades, the number of people who are over 85 years old has increased, too. Not only objective health status of the elderly, but subjective judgements of the elderly are also important, but about a half of the elderly in Korea evaluate their subjective health status to be relatively low level (KOSTAT 2017b).

Public provisions of medical care service for the elderly are divided into two categories, the National Health Social Insurance (NHIS) and a medical protection programme. While the NHIS is a contribution-based public health care provision for all citizens in Korea, the medical protection programme is a part of the NBLS. When receiving healthcare service of the NHIS, a user still bears $20 \%$ of the total treatment costs as a co-payment technically, but in practice one is more likely to bear about $46.6 \%$ considering the contributions (Won Seok-Jo 2006). The eligibility of the medical protection programme requires a means-test from the applicant and offers medical service for free, excluding a number of specific care services.

Meanwhile, the overall health care expenditure for the elderly has been increasing as the population in Korea has been ageing. The proportion of medical costs for the elderly with 65 and over of total fee paid by the NHIS has been continually increasing since the 2000s. 
In this regard, new measures to cover care cost for the elderly have been one of the most important contemporary questions in Korean society since the 2000s. According to the 2013 Household Income and Expenditure survey, elderly households in poverty bore the higher burden of health and medical care expenses, while caring and household service expenditures were more burdensome for elderly in the middle-income class compared to other income classes (KOSTAT 2013).

Against this background, the Long-Term Care Insurance (LTCI) for the elderly was introduced in 2008 after three periods of pilot projects. The LTCI is contribution-based social insurance like the NHIS. The expenditure of the LTCI is financed separately from that of the NHIS, though they share a management system regarding the collection of contribution as well as the assessments of the entitlement. Elderly of 65 years and over can be entitled to long-term care service after an assessment, and the recipients of the LTCI services are categorized into 5 groups according to the need of care assessed by inspectors of the NHIS. Only recipients with Grade 1 or 2 are entitled to choose between facility care services and in-home care services, others with Grade 3 to 5 are eligible for in-home care services only. When the LTCI was implemented in 2008, there were only three Grades, later the grading system has been reorganised into 5 Grades, and the percentage of the entitlement to the LTCI services has increased. In particular, Grade 5 was implemented specifically for the elderly with mild cognitive impairments. Service users of the LTCI share $15 \%$ of the costs for in-home care services and $20 \%$ for facility care services as copayments.

Compared to previous elderly care services, the LTCI is a more universal programme for its eligibilities based on the need for LTC instead of on a means-test. The numbers of people receiving services from the LTCI has continually increased over the years since its establishment. In 2009290 thousand (5.6\% of the total population with 65 and over) people received services over the LTCI, while in 2017 the numbers had more than doubled to 570 thousand (9.6\% of the total population with 65 and over) (NHIS 2018).

A more detailed explanation of the LTCI for the elderly programme will be given in section 2.4 (Family care helpers of the LTCI in Korea), which describes the delivery system of the LTCI and family care helpers as both a service provider and a service user. 
Apart from the NHIS and the LTCI, there is public social service provision offered by central and local governments, which have established senior centres social welfare centres for the elderly, and the centres offer educational and cultural programmes for leisure activities for the elderly.

Given that Korea faces the two-fold challenge of increasing needs for elderly care and decreasing care provision among family members, several studies have emerged on the status of care burden of family members and changes in attitude towards caregiving among family members (Kim Yong-Hee and An Jeong-Shin 2012; Kim Hye-Kyung and Namgoong Myung-Hee 2009).

\subsection{Family Relations in Korea}

This section explores how family and family relationships are formed and how family constellations affect elderly care in Korea. I begin by clarifying what I mean by the term 'family' in my study. I am focusing on relationships in adult life, so my work is not about care for young children. Instead, the family relationships I work within this study include parents-adult children and husband-wife relationships. The familism in Korea shows the characteristics of a patrilineal family system, including primogeniture, ancestral rites, son preference, responsibility for caring for the parents and keeping family values. Korean familism is supported by the concept of 'hyo (효, 孝)', which is a moral value based on blood ties and biological reciprocity and has become a moral foundation for caring for one’s old parents as a custom and institution. 'hyo (효, 孝)' as a moral value has meaning beyond a mutual exchange since the parents' presence itself has fundamental importance to children regardless of any kind of support they may receive from their parents.

The concept of 'hyo (효, 孝)' is one of the most important moral values in Korean society though, and parents do not always receive care from their children. Furthermore, it is also usual for the parents to support their children with, for example, money transfers or helps with caring for grandchildren, which means the relationship between adult children and parents are reciprocal (Jung et at. 2005). Besides, Kim Cheong-Seok and Kim Ik-Ki (2000) argued that it is essential for parents to establish exchange relations between themselves and adult children, since a situation where the parents are not capable of returning supports they receive from their children can have negative psychological impacts like feelings of guilt and shame as well as possibly depression. 
However, 'hyo (효, 孝)' lingers as an abstract moral value and is therefore not convincing in practice anymore since the value cannot reflect changes in the modern family structures and relationships. So, the concept needs to be reconstructed according to the present family situation.

The family has been the nucleus of the society in Korea regarding norms, customs, and institutions. Especially, the patrilineal stem family system has been considered as the ideal type of family in Korea. The patrilineal stem family is formed around the oldest son as the pivotal figure in that he lives with and supports his parents. To understand caring for the elderly parents by the oldest son's family, I focus on two different hierarchies among family relations, namely gender and birth order. The oldest son has priority over not only sisters but also younger brothers. This is because the oldest son has the responsibility to care for their old parents and family rituals for their ancestors. For this reason, the oldest son is usually supported by all family members; for example, teenage sisters of the oldest son often move to big cities to find a job in the light industry sector to support the livelihood of the family and the education fee for the oldest brother. While daughters care for their old parents in the sense of emotional labour, like affection for their parents, the oldest sons are likely to care financially in the sense of a family obligation (Kim Hye-Kyung 2013: 122). The oldest son's attitude towards caring for his parents can be understood as a moral responsibility as the oldest child in the family or a reciprocal behaviour in return for being supported by other family members. Apart from responsibility and reciprocal behaviour, the oldest son's care for the parents is also linked to his expectation of inheritance after the parents' death. In this sense, elderly care offered by the oldest son's family contains institutional attributes rather than emotional and relational attributes at the micro level.

Although the oldest son is in charge of the responsibilities for family values and cares for the parents in name, in practice his wife usually fulfils his obligation due to the gendered division of domestic versus gainful work - care work falling into the category of domestic work. The elderly care supplied by the oldest son's family shows that the traditional Korean care culture works based on patriarchal gender hierarchy. This stubborn familism in Korea hinders interactions among family members and intimacy in elderly care. As the person in charge of elderly care, the oldest daughters-in-law ${ }^{5}$ have been the group most often responsible for family care. Many studies on caregiving by the daughters-in-law in Korea

\footnotetext{
${ }^{5}$ In this study, the oldest daughter-in-law refers to the wife of the oldest son.
} 
argue that they care for their parents-in-law due to a family obligation in the patriarchal family system in Korea instead of affection and compassion for their parents-in-law. In this context, it is hard for the daughters-in-law to achieve autonomy within a family context where duty and obligation are more important than affection, and they are likely to experience conflict with the family members in care and to feel emotional and physical burdens (Kang Hyun-Sook et al. 1999; Kim Mi-Kyung 2000; Namgung Myunghee 2009; Seo In-Soon 2003).

Shin Kyung-Ah (2011) argues that elderly care supplied by the oldest daughter-in-law based on the patriarchal familism involves a major contradiction since the oldest daughtersin-law have to give priority to their parents-in-law over their own parents due to the patrilineal family system. This contradiction often leads the oldest daughters-in-law denying and resisting to support their parents-in-law (Shin Kyung-Ah 2011: 70). Moreover, in the extended period of cohabitation with the husband's parents in the sense of a patriarchal residence, the daughters-in-law are likely to experience the relationship with their parents-in-law in a negative way, and the conflicts between them can have a negative impact on the caring relationship later in life (Seo In-Soon 2003). Furthermore, the daughter-in-law may experience feelings of anger and guilt, as well as a resignation and abandonment towards caring for the parents-in-law (Kang Hyun-Sook et al. 1999). Anger and frustration with their situation may lead them to try to deal with the difficulties by legitimising strategies like asserting fatalism or considering the loss of family face (Yih Bong-Sook 2004).

Nowadays the norm of 'hyo (효, 孝)'has been increasingly changing as Korean society itself has been exposed to many social and economic changes. Two factors, in particular, illustrate the transition of care culture in Korea: one is a decrease of co-habitation, the other is a shift of the responsibilities as a major caregiver for the elderly from the daughters-inlaw toward other family members, mainly daughters and spouses. In the next section 2.3.1, I explain these changes in more detail and consider how they affect elderly care culture in Korea.

\subsubsection{Changes in family relations in Korea}

\section{Change 1: Decreasing cohabitation with adult children}


One of the traditional family systems in Korea is that of patri-local residence. This refers to the family system in Korea in which a married couple resides with or near the husband's parents. The oldest son and his family live with his parents and siblings in paternal line to care for them until the end of the parents' life, following the maxim of the oldest son's obligation. Therefore, cohabitation has been known as an indicator of support for the parents. Until the 1990s, the tradition of a patri-local residence had remained as the most prevalent model and the oldest-daughter-in-law cared for her parents-in-law, even if her husband lived remote from his family for his job. However, the rate of the cohabitation has decreased since the 1990s, and it has become more common for the adult children to live separate from their old parents, even though they live in the same district (Kim Eun-Hee 1995).

As Korean society has modernised, many young people leave their hometown and move to a city to find a job in the industrial sector. This tendency has led to changes in the family structure for example, the oldest son who works in the rural area may follow the traditional care culture. Han Gyung-Hae and Soon-Duck Yoon (2000), who analysed changes in the meaning of being the oldest son in the course of industrialisation in Korea, argued that by modernising and industrialising Korean society the concept of the patri-local residence of the oldest son's family with their old parents did not fit in with the son's other obligation anymore. They found two different analytical types of oldest sons. One left his hometown to acquire an improved social status which also linked with the elevation of the family's social status, the other type stayed in the rural area to care for their parents and accepted the lower social status that comes with it in industrialised society.

But not only the adult children's attitudes towards cohabitation with their old parents in the sense of family obligations changed, but the views of the parents themselves did too (Kim Hye-Sook et al. 2005). Indeed, more parents want to live separate from their adult children, and a study found that the parents who live alone were not less satisfied with their life compared to those who live with the adult children (Shin Kyung-Ah 2011). However, when the parents fall ill and as a consequence cannot live on their own anymore, the adult children are likely to take the parents to the big. They take their parents to live with them because it is easier to access hospitals. The change of the residence for the elderly form rural areas to cities is an alternative to personal care for the old parents. Moreover, the cities offer better access to medical services compared to rural areas (Han Kyung-Hye and Yoon Soon-Duck 
2000). On the other hand, the abrupt changes in the residence are not a proper choice for the elderly in the sense of the 'ageing in place'. Lee Hyunsook (2011) criticises this modernised Korean care culture of the elderly being moved to live with the adult children as not being appropriate from an elderly-centred perspective in that for the frail elderly, especially those with cognitive impairments, it could negatively affect their health condition. A significant number of the elderly are in a dilemma where they want to stay at home and be cared for by family members until the end of life on one hand, but on the other hand they understand that following the traditional care model may not be possible given the context of the modernised and industrialised Korean society. Therefore, they ask for care offered by the state as a supplement to informal family care (Shin Kyung-Ah 2011: 81).

Furthermore, since the 1990s family size has decreased into a nucleus family, and while the concept of cohabitation may remain, it is with some changes in the reasons behind why the adult children are willing to live together with their old parents. More adult children decide to live with their old parents because of instrumental needs like support with childcare and house (Kim, Hye-Sook et al. 2005; Jung Kyunghee et al. 2004; Han GyungHae and Yoon Soon-Duck 2000). The changes in the living arrangements show an emergence of a diversity of contemporary family concepts in Korea rather than general family disorganisation (Namgung Myung-Hee 2009: 64).

\section{Change 2: From daughter-in-law to a daughter in intergenerational care}

As the importance of the role of daughters-in-law in elderly care has diminished, spouses and other children have been substituting as caregivers for the elderly. The proportion of spouses who are major caregivers has increased from 37.0\% in 2004 to 53\% in 2011, while the response of the adult children and their spouses decreased from $57.6 \%$ to $41.3 \%$ at the same time (see Table 2.3).

Table 2.3. Major caregivers for the elderly in need of care in Korea

\begin{tabular}{crrr}
\hline & & \multicolumn{2}{c}{ (Unit: person, \%) } \\
\hline Spouse & 2004 & 2008 & 2011 \\
\hline Adult children and their spouse & $297(37.9)$ & $424(53.2)$ & $550(53.0)$ \\
Other family members & $451(57.6)$ & $356(44.7)$ & $428(41.3)$ \\
\hline
\end{tabular}




\begin{tabular}{cccc}
\hline & 2004 & 2008 & 2011 \\
\hline Total & $783(100)$ & $796(100)$ & $1037(100)$ \\
\hline Note * Adult children and their spouse: sons and their spouse, daughters and their spouse,
\end{tabular}

Note: * Adult children and their spouse: sons and their spouse, daughters and their spouse, ** other family members: grandchildren and their spouse, siblings and relatives.

Source: KIHASA, Living Profiles and Welfare Service of Older Persons in Korea', Survey 2004, 2008, 2011.

Unlike Western countries where mainly daughters have been expected to care for their parents in the sense of affection (Lewis and Meredith 1988), daughters-in-law have been a major caregiver for their parents-in-law in the sense of traditional family obligations in Korea where the patriarchal and gendered norms for domestic work divisions are dominant. There have been several studies on the care experiences of daughters-in-law who care for their parents-in-law, but there has been little research into the experiences of sons or daughters who care for their parents.

Recently, there emerged several studies on trends towards a bilateralization of kin relationships in Korea (Cho Cheung-Moon 1997; Han Gyung-Hae and Yoon Sung-Eun 2004). The concept of the bilateralization of kin relationships refers to a trend of both paternal and marital directions of kin relationship instead of the traditional Korean kin system of the patrilineal logic (Cho Cheung-Moon 1997). However, the trend of the bilateralization in Korea differs from that in Western societies in that the Korean bilateralization is a reflection of the asymmetric family relationship based in patriarchal authoritarianism. Specifically, there are differences in the forms of support adult children receive, depending on if they belong to the paternal or the maternal side of the family. While economic support usually comes from the paternal family who place importance on family's obligations and rituals, emotional support and childcare come from the maternal family, mainly, the wife's mother (Lee Jae-Kyung 1999; Han Min-Ah and Han Kyung-Hye 2004). These differences illustrate that the Korean bilateralization is a result not of equalisation between men and women in the family but of increased needs for maternal kinship as a new social resource in contemporary Korean society (Namgoong Myoung-Hee 2009: 66).

In the course of this trend of bilateralization, several studies have been conducted on the relationship between married daughters and their parents regarding elderly care. A recent study suggests that elderly living with their daughters' family are likely to feel more 
happiness and experience fewer conflicts compared to the elderly living with their sons' family (Han Gyung-Hae and Yoon Sung-Eun 2004). The increase of elderly care offered by daughters can be explained by several reasons. Firstly, as the traditional care culture has weakened, the daughters are more likely to have a strong emotional bond with their parents compared to sons. There has been placed increasingly more importance on emotional functions of the family instead of ritual functions, and as a result of downsizing family, there are more families who have only daughters. In addition, women now also have the same opportunity for education as men. These factors lead to an increase in elderly living with their daughters' family instead of their sons’ family.

As changes in later life and family relations in Korea, research trends have changed. Section 2.3.2 will show the state of the art on the elderly care family practice.

\subsubsection{Research trends in elderly care practice among families in Korea}

This section explores research trends on family care practice in Korea where rapid industrialisation and urbanisation since the 1960s have led to major changes concerning later life, family structure and care culture; for example, a decrease in cohabitation of married adult children's families with their old parents as well as an increase in women's participation in the labour market. Unlike European societies, Korean society did not start to discuss these issues regarding population ageing and changes in the family before the 1990s. Although the number of persons in Korea who are 65 and over is not high yet compared to most European countries, the speed of ageing is rapid. Korea entered the categories of ageing society and the aged society in 2000 and in 2017 respectively.

Apart from these demographic changes, there emerged new policy approaches due to the combination of an economic crisis and a political regime change towards a more liberal regime in the late 1990s. Against these backgrounds, the new government pledged to implement a new programme for care, and there was a discussion on the manner of public provision for elderly care and the relations of the new elderly care programmes with the family. In 2008, the LTCI was implemented, which meant that family caregivers could choose different kinds of care settings. For example, Yang Nan-Joo and Choi In-Hee (2013) reported that family caregivers selected different combinations of formal and informal service types whereby family caregivers play diverse roles, including service user, informal caregiver, and certified care provider (FCH). Therefore, I will divide the research trends 
into elderly care practice among families into three periods: i) informal family caregiving (until the late 1990s) ii) establishing a new elderly care programme (the 2000s) iii) various settings of family caregiving (after the implementation of the LTCI in 2008).

\section{Informal caregiving (until the late 1990s)}

Although the rate of three-generational cohabitation in Korea has been decreasing since the 1980s, the traditional care culture offered at home by the first son's family remained in the sense of the son's obligations until the late 1990s. When the elderly got frail, their adult children lived together with their frail elderly parents who cannot live independently any longer. Most of the studies on family care practice in the 1990s focused on family caregiving in an informal setting and the importance of family care values and care norms (Cho Myoung-Ok 1999; Kim Kwuy-Bun and Lee Kyung-Hee 1998; Lee Kang-Oh 1999). These studies came from a perspective that regarded elderly care as a family issue which had to be solved at home by family members.

In this period, care was regarded as an expression of people's respect and love for their parents. Individually, although the care responsibility originated from the first son's obligations, care practices, in reality, were mostly performed by the first daughter-in-law, meaning the wife of the first son. Kim Hyunjoo (2001), in her analysis of first son's families in the 1990s, argued that the first son in the family is able to work only when his wife commits to the son's old parents and family. Chang Kyung-Sup (1992) pointed out a cultural lag in Korea in that society still puts care responsibilities onto a family in the sense of the traditional care norm, even if the family itself is not sufficient to deal with elderly care. Although there was research on the changes in family structure and values, there had not yet emerged arguments on necessities of 'socialising care' for the elderly.

\section{Establishing a new elderly care programme (the 2000s)}

Meanwhile, Korean society experienced the Asian financial crisis in 1998, which meant that the family-centred support system was weakened. To overcome the financial crisis, the government has started to move towards deregulation and privatisation, which led to diminished family wages and to an increase in women's economic participation. Since 2000, a combination of interconnected demographic, economic and political factors have led to unprecedented social policies, especially for frail elderly people given that the rate of the old population who were 65 and more in age reached 7\%, and Korea became an ageing society in 2000 . 
The government started to plan a public programme for LTC services in the 2000s, so, there emerged research on care arrangements and a formal care provision system for the elderly. Before the introduction of the LTCI in 2008, studies on family caregiving were mainly focused on the issue of family conflicts in relation to care for the elderly among family members. Notably, there were studies on the care burdens and stress daughters-in-law experienced in their role as carers (Seo Insoon 2003). The analysis was mainly levelled at cultural differences in approaches between the daughters-in-law and the frail elderly, and there are studies on the necessities of public provision in the LTC service for mitigating the care burdens of families (Huh Ra-Keum 2006; Kang Hyun-Sook et al. 1999; Seok Jae-Eun 2006).

However, while the necessity of public provision for the elderly emerged in Korean society and measures started to be taken, traditional care norms, according to which the family should take all the responsibility for elderly care in the sense of children's obligations, were still co-existent. Huh Ra-Keum (2006) argues that the government and the society in Korea did not arrive at the forming of a social consensus on 'socialising care', in which the central and local governments had to establish a public system of care provision to share the responsibility for elderly care (Huh Ra-Keum 2006: 129) Shin Kyung-Ah (2011) also points out that the elderly who are cared for outside the family have no normative resources for justifying and accepting their life in the care facility given that they live in a society without any public discourse about them.

Meanwhile, there were also studies focusing on the relational aspects of elderly care by family members. However, in these periods, the studies concentrated more on the caregiver's autonomy instead of the value of family, which means the family care for the elderly becomes an issue not only about pursuing a value of family at a collective level in family and society but also about quality of life and relationships between two individuals. For example, Namgung Myunghee (2010) suggested the notion of relational autonomy which describes a family caregiver's quality of life and their relationship with the elderly relatives they care for.

\section{Various settings of family caregiving (after 2008)}

As the LTCI was implemented in Korea in 2008, elderly care has been one of the most important issues in social policy. There emerged research on influences of the LTCI on the family (Chang Kyung-Sup et al. 2015; Ham Inhee 2014; Ham Sunyu and Hong Bageui 
2017; Kong Seonhee 2013; Lee Jaerim 2015; Sohn, Yong-Jin 2012), the experiences of care workers (Jegal Hyeon-Suk 2009; Jun Byungyu 2010; Kim Song-Yi 2012; Kweon Su Hyun 2013; Ryu Im-Lyang 2017), and the relationships between care workers and family members (Chon 2015).

With regard to the influence of the LTCI on family care, several studies pointed out that although public care provision had been introduced in Korea, elderly care policy still remained family-centred (Cho Hye-Young et al. 2015; Lee Jaerim 2015; Shin Kyung-Ah 2011) Lee Jaerim (2015) points out that although formal care provision had expanded in Korea since 2000, familialism is still embedded in the care policies. Shin Kyung-Ah (2011), in her analysis of the experience of the elderly in a care facility, noted that the elderly in Korea still thought that care at home was the best kind, but they could not avoid entering a care facility because they also knew that care responsibility could not belong to the family alone anymore. She argues that as the family cannot take all the responsibility for elderly care, society has started to share the care responsibility.

Another significant trend in family care is the increase in spousal caregiving (Choi In-Hee and Kim Jung-Hyun 2013; Han Gyoung-Hae and Lee Seo-Youn 2009; Lee Mee-Ae 2006; Paik Jina 2015; Yoon Hyun-Sook and Ryu Sam-Hee 2007). This increase in spousal care is in line with the decrease in the cohabitation with adult children (see section 2.3.1). Scholars point out that spouses who care for their partners perceived care as an expansion of marriage life, meaning their experience of care practice differs from that of adult children. Paik Jina (2015) points out that wives who care for their frail husbands put emphasis on collective family values and they accepted a role derived from traditional gender norms. While the wives experienced physical and economic challenges in caring, they also experienced intimacy with the elderly and strengthened bonds among family members.

Although the FCHs are understood as a significant element that shows the family-centred care culture in Korea, there has been little research on the FCHs themselves. While there has been research on the informal family caregivers and care work after the implementation of the LTCI in 2008, there has been little focus on the experience of the family care helpers, who are licenced family caregivers for the elderly. 
There was research on the FCHs is about patterns of LTCI services provision compared to non-familial care workers (Lee Yun-Kyung 2010, Hong Sung-Wook 2011), but these empirical studies mainly aimed to investigate whether the FCHs ask for unnecessarily high fees, which were thought to hinder the sustainability of the LTC finances.

Few studies focus on the experience of the FCHs (Choi In-Hee and Kim Jung-Hyun 2013; Jeon Byeongjoo and Kim Hyunsoo 2016; Lee Min-Sook 2012; Yang Nan-Joo 2013). Lee Min-Sook (2012), in her investigation of the experience of the FCHs, argues that most of the FCHs who were interviewed were committed to caring for their family members. In other words, not all the FCHs tried to misemploy the payments for elderly care and service regulations. She also noted that the family caregivers often hesitate to become paid family caregivers since they have problems accepting the thought of being paid for what has been considered as a moral obligation to the family (Lee Min-Sook 2012: 61). Choi In-Hee and Kim Jung-Hyun (2013) point out that most family caregivers were likely to decide to become FCHs due to economic considerations, but the payments were not enough to maintain their household, which is why they felt they had to demand higher fees for their care work (Choi and Kim 2013: 176).

\subsection{Family Care Helpers (FCHs) of the LTCI in Korea}

The overviews on later life, family relations in previous sections 2.2 and 2.3 provide the backgrounds and context of the appearance of the FCHs in the LTCI in Korea. Based on these overviews, this section explains how care responsibilities for the elderly are divided between the state and the family by focusing on the status of the FCHs, more specifically by tracking changes in the regulations of the LTCI on family caregiving from its introduction to the present. Firstly, I assumed in Chapter I that the FCHs are situated between formal and informal status. This assumption stems from the FCHs peculiar position in the system of the LTCI as being users and providers of services at the same time. In addition, I aim to trace the trajectories of changes in payments for the FCHs since the trajectories would give hints for the arrangements of care responsibility for the elderly between the state and the family.

Section 2.4.1 will explain the relevant institutional dimensions of the LTCI, including the background of its introduction, its financing and the service delivery, which consists of three agencies (an insurer, a user, and a provider). In the service delivery, the FCH is a user 
and a provider at the same time. In section 2.4.2, I trace the trajectories of the changes in the status of FCHs. I focus on the uncertain situation regarding the status of FCHs and examine their position between the paid labour market and the domestic area.

Care for dependents in ageing societies has become one of the most important issues in Asian countries. Many societies in Asia have implemented elderly care programmes to deal with elderly care as a social risk caused by demographic changes like increasing life expectancies and low fertility, as well as by social changes in family structures and care culture. Due to these changes, the perspective on elderly care should move from the domestic arena to the social arena (Ma Kyung-Hee 2011; Moen and DePasquale 2017). The concept of 'social care' (Daly and Lewis 2000) is helpful to understand the multifaceted characteristics of care in relation to the welfare state which arranges care responsibilities among three agencies: state, market, and family. This concept of 'social care' will be reviewed in detail in Chapter III.

In 2008, Korea introduced the long-term care insurance (LTCI) scheme, which was established separately from the national health insurance (NHI) and offered social services only for the elderly and those who have geriatric diseases. The new social insurance scheme was planned and enforced by the central government, and it raised several issues regarding the traditional care culture and the welfare system in Korea. Before the introduction of the LTCI, elderly care in Korea had been offered only in a domestic setting in the context of the first son's obligations. Thus, the social infrastructure for elderly care was insufficient due to this still family-centred perspective of care culture. For this reason, the government decided to get former informal family carers involved in giving them the option to become qualified care helpers, called family care helpers (FCHs hereafter). This government's policy choice could also be understood as emphasising cost containment, in that the government made a compromise between the costs and the responsibilities for elderly care in involving family members, who had already taken responsibility for elderly care. Indeed, the LTCI provides a lower level of compensation for home visit care services offered by FCHs, compared to that offered by non-familial care helpers.

Against this background, it is necessary to be clear about the criteria for payments for the family carer. It is therefore important to set out the status of FCHs, who can be placed between the formal and informal areas of care for the elderly. Earlier studies on the LTCI and family carers in Korea have focused on one side of the characteristics of carers, which 
means the carers have been sorted into formal or informal, paid or unpaid (Oh Eunjin and Roh Eunyoung 2010). Recently, there have been several studies focused on the two-sided or multi-faceted characteristics of FCHs in the LTCI in Korea (Lee Min-Sook 2012; Yang Nan-Joo 2013). However, most studies have not put much emphasis on changes in payments for the FCHs' caregiving.

\subsubsection{Family care helpers (FCHs) of the LTCI in Korea}

This section explores what factors led to the introduction of the LTCI in Korea in 2008. An increase in health costs for the elderly, as well as changes in families and care attitudes, were factors in the introduction of this programme. In addition, the government considered the LTCI as an economic growth engine which would create employment in the social service sectors. Firstly, the LTCI was introduced as a countermeasure in response to increasing elderly health care costs in the NHI system caused by ageing of the Korean population. The National Health Insurance Service (NHIS) estimated that the proportion of elderly care costs would rapidly increase, from $8.2 \%$ of total health costs in 1990 to $29.9 \%$ in 2008 (NHIS 2009). Another factor in the introduction of the LTCI were changes in family structures and care attitudes in the country. As women's education levels increased, the percentage of women's economic participation increased with them. The attitudes towards caring for the elderly have also changed, from family obligations based on a traditional care culture towards an attitude towards new social risks in which state, society, and family share the burden (see section 2.3). Thirdly, the government considered the elderly care sector as a new growth engine. The Ministry of Health and Welfare (MHW) (2005) predicted that the new insurance programme would create about 2,000 new jobs in 2007, and about 4,000 annually thereafter. Because of the job creation effect, the insurance was supported by other ministries, such as the Ministry of Planning and Budget, even though the insurance would impose a financial burden (Park Ha-Jung 2008: 55). Therefore, the government tried to entice private providers to enter into the care industry. Several scholars have criticised the government's attempts in this regard since they have led to a negative impact on the service delivery system and the working conditions of care workers (Jegal Hyeon-Suk 2009; Seok Jae-Eun 2010).

Against this background, the LTCI in Korea has peculiar characteristics. Firstly, the LTCI has the characteristic of having low contributions and low benefits. Historically, Korean social welfare expenditure has been low compared to other Organisation for Economic Co- 
operation and Development (OECD) countries. For example, the Korean government spent only $10.1 \%$ of gross domestic product (GDP) on social welfare expenditure in 2012, as compared to the mean among OECD countries of 22.1\% of GDP in 2011 (OECD 2015). Another feature of the LTCI is allowing former informal family caregivers to come under the status of service providers - given those family caregivers are qualified as FCHs. This measure arose due to the Korean traditional care culture. As LTC services in Korea were very limited until the 1990s, the public long-term care provision was only offered to the elderly in poverty, according to the Protection of Minimum Living Standards Act (see section 2.2.3). Most elderly were cared for at home by family members - mainly the first daughter-in-law. To avoid a radical interruption in the supply of care provision, the government decided to involve the former as buffers in gradual institutional changes in elderly care. Lastly, because of this traditional care culture for the elderly, there was little infrastructure for care provision in both public and private areas (Peng 2011: 905). Elderly care services in the public sector and in the paid market were very limited in Korea. The government has eased restrictions on manpower requirement criteria and equipment standards for LTC provision for fear of 'imaginary criticism' for a deficiency in the care supply (Seok Jae-Eun 2010: 39). Therefore, the government tried to encourage providers in the private sector to enter the new paid care market.

Although the LTCI has a separate fund system from the NHI, the LTCI and the NHI share a service delivery and management system. The NHIS collects both contributions but manages the funds separately. In addition to the funding system, the LTCI uses the NHI's infrastructure and manpower regarding the assessment and grading of applicants for the entitlement to LTC services, as well as qualifications for providers like care agencies and care helpers. The LTCI is financed by contributions, the rate of which is determined by the NHI contribution rate ${ }^{6}$. The LTCI has aspects of intergenerational contract in that the economically active population pays contributions for the elderly who do not participate in the labour market (Jegal Hyeon-Suk 2009: 217). In addition to the contributions, state and

\footnotetext{
${ }^{6}$ In 2016, the LTCI contribution rate was imposed at $6.55 \%$ of the NHI contribution rate. The contribution rate of the NHI is calculated differently according to the employment status. In the case of the employee insured, both the employers and employees equally pay their contributions according to their income, while the self-employed are responsible for $100 \%$ of the contributions according to evaluated income based on a mixture of their assets and cars. Civil servants and state school teachers, as well as state, local governments, and private schools, co-pay 50\% each according to their incomes, and in the case of private schools, the school pays $30 \%$ and the state $20 \%$. These separated collections are an old legacy of the administrative form of the NHI scheme. Korea had a society-based and decentralized system until 1999. In July 2000, all funds have been integrated into the NHIS.
} 
local government share the responsibility for financing regarding management costs and contributions for recipients of medical care public assistance. The recipients of the LTCI also share a partial payment, which is called an individual co-payment, the rates of which for in-home care services and facility care services are $15 \%$ and $20 \%$ of the total costs they use, respectively.

As mentioned above, the Korean insurance system has the characteristics of low contributions and low benefits (Jegal Hyeon-Suk 2009) mitigating elderly care costs constraints was one of the primary purposes of the LTCI, so that the state sets the insurance as a low contribution and low services system. In this circumstance, the inclusion of former informal caregivers was necessary to contain care costs (Im et al. 2011; Kim Keun-Hong 2013).

To analyse where the FCHs are situated in the LTCI, one should first investigate the service provision system. The concept of social welfare service delivery refers to a structural series of connected processes among a service producer, a user, and a provider (Gate 1980; Kim Soon-Yang 2007), and subjects who are involved in the LTCI can be categorised into three groups: an insurer (NHIS), users (the elderly and FCHs, as their family members), and providers (care agencies and FCHs). The LTCI has a centralised fund system, and the NHIS, as an insurer, carries out an examination of qualifications for entitlement, assessment and collection of contributions, grading of applicants, remuneration management, financing, and research (see Figure 2.1).

Figure 2.1. Role of the insurer in the LTCI in Korea

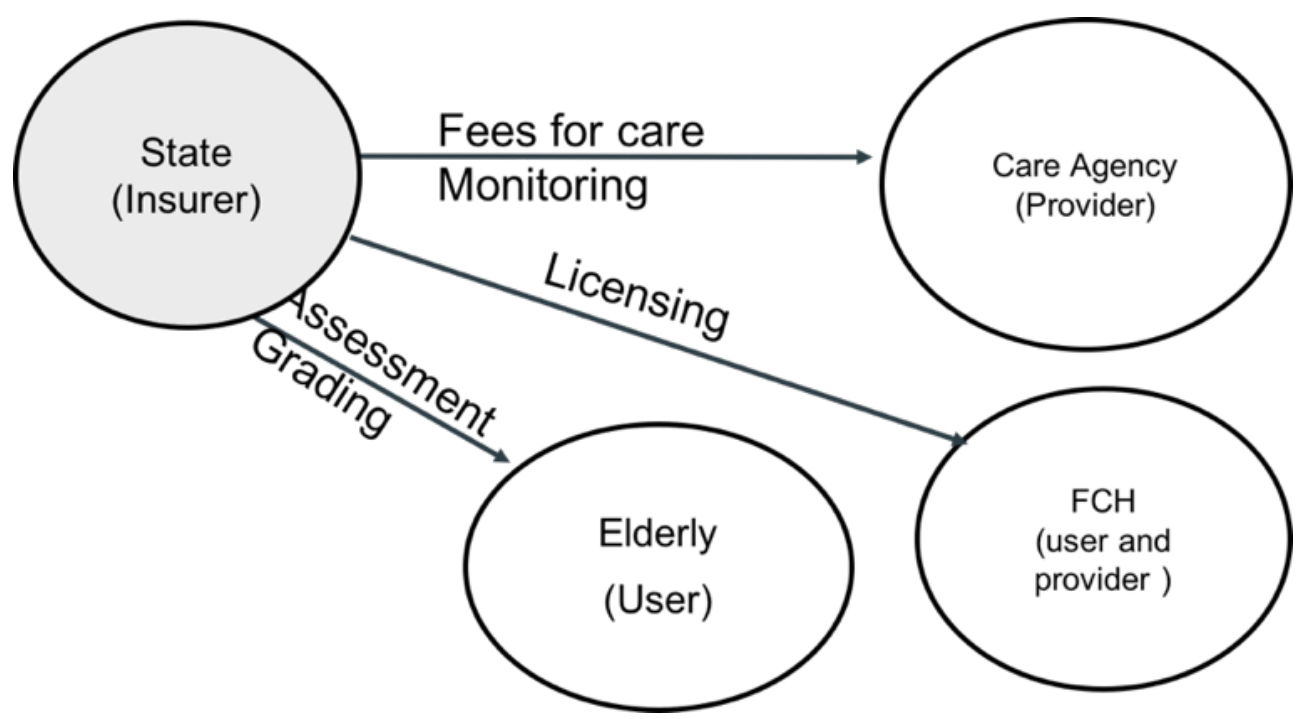

Source: Author 
A care agency, as a provider, mediates relations between the insurer (NHIS) and users. Whereas the insurer manages regulations regarding assessment and grading of applicants for the LTCI, as well as licensing and qualification of service providers, care agencies take charge of actual LTCI service provision. A social worker or a medical licence holder can register with a care agency (usually called a "care centre” in Korea) with requirement criteria of facilities and manpower. The care agency makes contracts with both sides: users (service contract) and care helpers (working contract). The care agency also supervises the care helpers and reports the service provision of the recipients to the NHIS (insurer) monthly, to receive payments for care. The LTCI services are, in principle, in-kind benefits - most in-home services flow through the care agency (see Figure 2.2).

Figure 2.2. Care agency in the service delivery in the LTCI

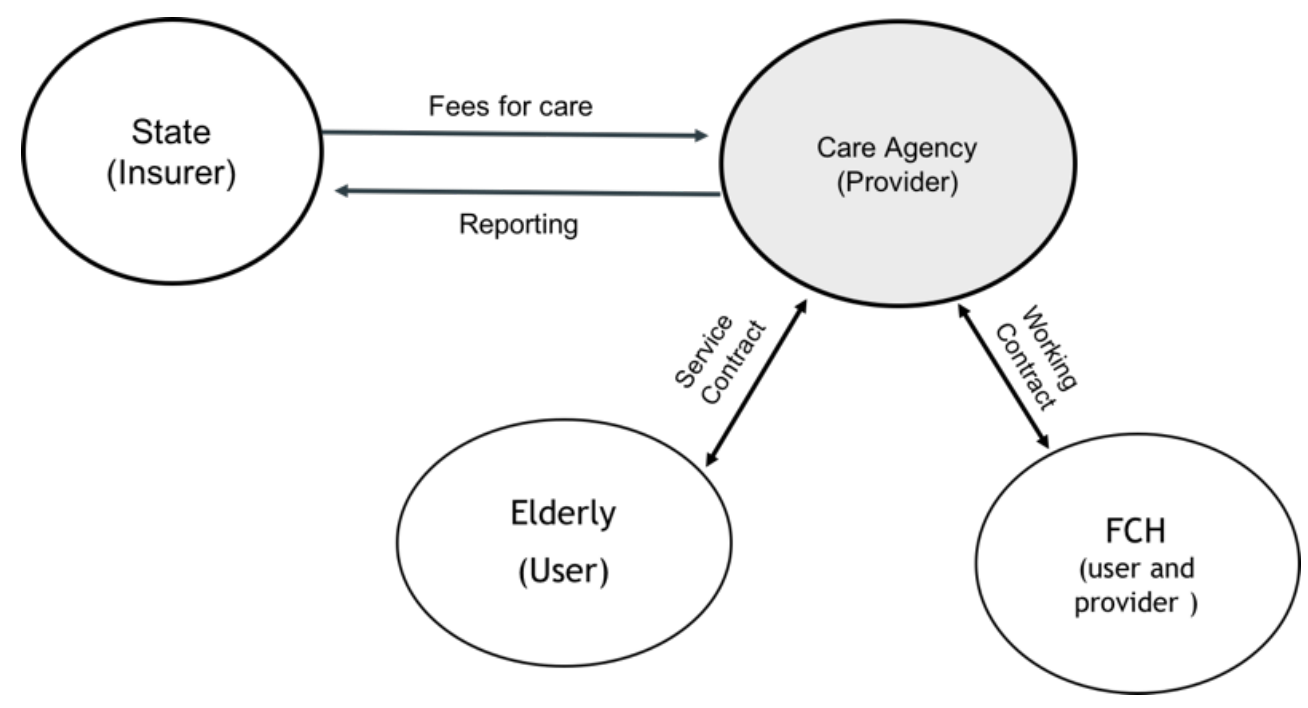

Source: Author

In the service delivery system of the LTCI, the FCHs can be in both positions, the position of a user and one of the providers, and there are different sets of choices regarding FCHs' care for their family members in the service delivery of the LTCI. The state, however, divides them into two and puts them in the paid care market, i.e. the elderly and the informal caregiver should separately proceed to access the LTCI services; the elderly should be entitled to the LTC service, and the FCH should obtain the licence for a care helper. To see the status of the FCH more clearly, two aspects of the status of the FCHs should be explained: access to the service as a user and the qualification procedure as a provider.

To be a beneficiary of the LTCI, one must meet several requirements. Although all those insured under the NHI are eligible for the LTCI and pay contributions, the beneficiaries of 
the LTCI must be elderly, aged 65 and older, the only exception being people under 65 who suffer from geriatric diseases. To receive LTC services, they are required to go through the processes of applying, grading, and making a contract with a provider. To apply for assessment, the elderly in need of LTC should prepare an application and a doctor's referral slip (medical certification). Then, the LTC Needs Certification Committee evaluates the status of the applicant's physical and emotional health according to activities of daily living and instrumental activities of daily living. If it deems the applicant has difficulty taking care of their daily life needs on their own for over six months, based on the result of the assessment the applicant is entitled to be a recipient of the LTCI (see Figure 2.3).

Figure 2.3. Process of assessing LTC recipients

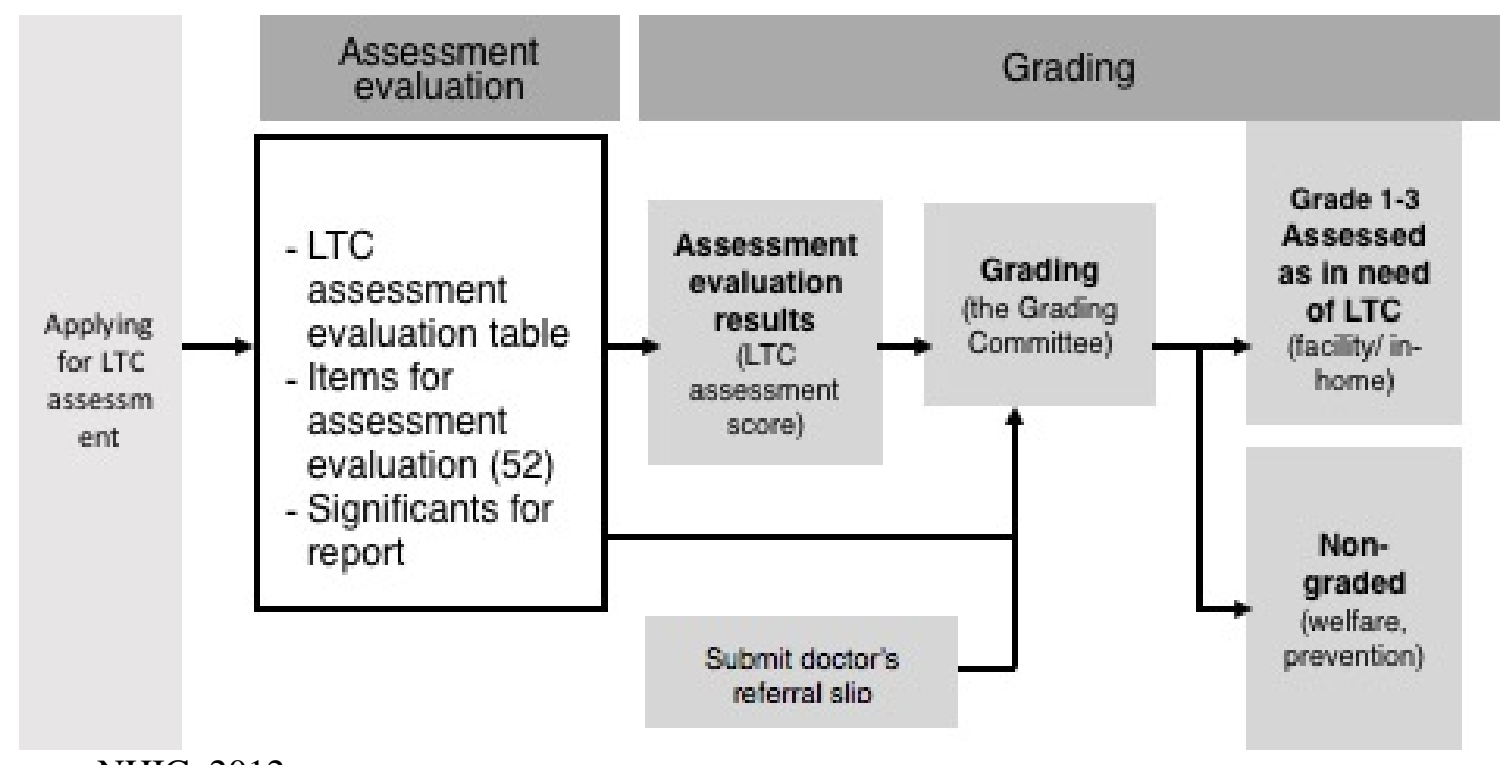

Source: NHIC, 2012

The recipient can select services regarding types of care provision as well as service providers in both the public and private sectors. The in-kind benefits are mainly supposed to be provided in forms of services in the LTCI, while cash benefits require strict preconditions and so are rarely used in Korea. The services can be categorised as in-home care and facility care, according to the location of the care provision. In-home care comprises home visit care, home visit bathing, home visit nursing, day and night care, short-term care, and the provision of welfare equipment. The recipient can also select care facility services which help maintain and improve the elderly person's physical and mental health for an extended period in an LTC facility, except for hospitals specialising in the treatment of the elderly. 
To be a FCH, a family caregiver follows the same procedure of non-familial care helpers in the LTCI. One becomes a care helper by taking an exam and obtaining a licence, and by making a contract with a care agency. The FCH remains under the supervision of the agency to be compensated by the LTCI. To get a licence as a care helper in the LTCI, one must complete a course of study presented by education institutes and pass the exam for the licence. The government has opened the educational institutes to market forces and has increased the role of private institutes in the care system (Jegal Hyeon-Suk 2009).

After getting the licence, the FCH makes a contract with a care agency. The type and hours of care services are specified in the contract, and the agency assigns a care helper to the recipient according to the contract. The working contract between the agency and the care helper regulates the working conditions, and the centre manages the working schedules of the helpers and gives a report on their caregiving to the NHIS for the calculation of monthly payments (see Figure 2.4).

Figure 2.4. The service delivery of home visit care after getting a licence

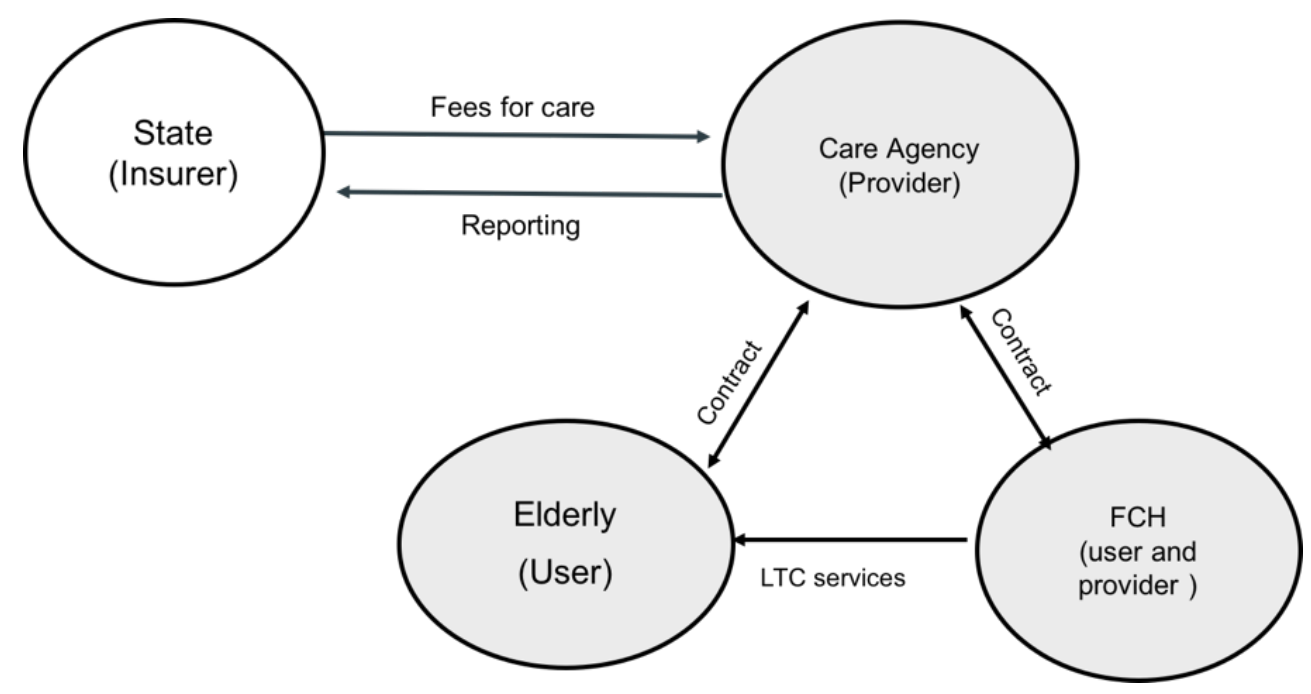

Source: Author

\subsubsection{The trajectory of changes in payments for in-home care service offered by a FCH}

When planning the LTCI, the state did not anticipate the explosive increase of family care helpers (FCHs). For this reason, the regulation on the payments for the FCHs were provided and amended ex-post. The payments for visit home care by a care helper are calculated according to hours they offered. While a single rate is applied to all care helpers regardless of family relations between the caregiver and the elderly, the recognised (paid) hour for the 
care by the LTCI differs between them. For example, even though a FCH offers care for the elderly at home around the clock, the LTCI pays for the recognised hour, which is predetermined by the regulations. In this sense, I assume that the changes in the regulations on the recognition hours would indicate the arrangement of the care responsibility between the state and family.

To trace the trajectory of changes in the status of the FCH, I investigate the government legislation on, and amendments to, the LTCI. For an analysis on changes in payments for the FCH, I set out three questions, which represent three dimensions in an analytic framework which covers the dynamics of elderly care arrangements among state, market, and family:

1. How do the legislation and amendments of the LTCI define the scope of a family member's responsibility for a recipient of the LTCI? The scope would suggest who is primary or partially responsible for the frail elderly person in the LTCI.

2. At what level can the FCH be formally recognised by the LTCI, compared to payments of non-familial care helpers? Since the introduction of the LTCI, the payments for in-home care services offered by the FCH, and the level of time recognition has changed according to the notifications of the MHW. The level represents how the care responsibility is shared between family and state.

3. Concerning entering the paid labour market, how is the FCH controlled and regulated by the state? The regulation represents the status of the FCH, who is located between the paid and unpaid areas of elderly care.

Although there have been several minor reforms made to payments for in-home care services provided by FCHs, the most important amendment that had an impact on the status of the FCHs is notification No. 2011-72 of the MHW on 29 June 2011. Therefore, I divide the period I analyse into two sections: before and after the enforcement of the amendment of notification No. 2011-72 of the MHW.

\section{Initial regulations on payments for family caregiving}

An initial regulation on payments for family caregiving was vague, and the definition of a family member who is primarily responsible for the frail elderly person differed from the present one. Once an individual had obtained a licence as a care helper and made a contract with a care agency, she or he offered in-home care services to the recipients of the LTCI regardless of whether family relations existed between caregiver and care receiver. However, there were some differences in the level of time recognition for their caregiving 
according to family relations and their living arrangements. According to notification No. 2008-66 of the MHW, when the FCH cohabitated with a recipient of the LTCI, payments for home visit care services offered by the cohabitated FCH were recognised only up to 120 minutes per day, while the services offered by a non-familial care helper were recognised up to 240 minutes per day. The reduced payments, however, were applied according to the living arrangements: whether the $\mathrm{FCH}$ and the recipient lived together or not. In other words, a FCH who lived separately from the recipient could be paid for their caregiving for the elderly up to 240 minutes per day. At the time of the introduction of the LTCI, even though the government presumed that the family should take on the primary responsibility for care for their elderly in the context of the principle of subsidiarity, the concept of obliged family members was limited only to the cohabited family members according to Civil Code Article $779 .^{7}$

In the initial period of the LTCI, both the kinship and the living arrangements of caregiver and receiver were the main criterion since the government assumed that some of the services the LTCI provided consisted not only of physical support for the elderly but also domestic work for the elderly, which was hard to separate from that for the whole household, like dish-washing, laundry, and cleaning the house, and these household chores have to be in charge of the co-habitant family instead of the state's.

This tendency showed that the government tried to involve former informal family carers in the LTCI programme since the infrastructure and workforce in the social service were so limited at that time, as the Korean traditional care culture was based on Confucianism. If the family carers had been excluded from the LTCI, this could have hindered the development of the infrastructure in the elderly care service market (Yang Nan-Joo 2013; Seok Jae-Eun 2010; Jegal Hyeon-Suk 2009). Moreover, the Roh Moo-Hyun administration considered the social care service sector as one of the new economic growth engines in an ageing society so that the administration mainly focused on expanding the elderly care market and providing employment (Park Ha-Jung 2008). This attempt of the administration

\footnotetext{
${ }^{7}$ Civil Code Article 779 (Scope of Family Members)

(1) Family members shall consist of the following persons:

1. The spouse, lineal blood relatives, and brothers and sisters;

2. Spouses of the lineal blood relatives, linear blood relatives of the spouse, and brothers and sisters of the spouse.

(2) In the case of paragraph (1) 2, it shall be limited to the case where they share living accommodations.
} 
was bound up with the interests of agents in the care market place, including care education institutions and in-home care agencies.

In addition, the Korean traditional care culture also made it possible for the society to accept the limited payments for the cohabited FCHs. Elderly care had traditionally been considered a sign of the filial piety of the oldest son (see Section 2.3), while the wife of the son was responsible for the practical care labour. As Korean society has industrialised, adult children have been less likely to cohabit with their parents than in the agricultural era, but the private transfer in the context of an intergenerational contract lingered, as compared to other industrialised countries (Peng 2011).

Indeed, there has been a drastic increase in the number of FCHs since the introduction of the programme - greater than the LTCI had expected. KIHASA (2010) reported that former informal caregivers were likely to become FCHs instead, receiving cash payments for family caregiving, since the requirements of the cash payments were very strict and limited. The number of recipients who received care payments for family care steadily decreased, from 1,004 persons in June 2009 to 763 in June 2010. In contrast, the number of recipients who received in-home care services by a cohabited FCH has continually increased, from 2,689 persons in December 2008 to 39,966 persons in June 2010, which accounted for $26.3 \%$ of the total recipients who received in-home care services of the LTCI (KIHASA 2010). As the number of recipients who receive the services by FCHs increased, the amounts of payments for the services also increased, from $1.2 \%$ of total payments for home visit care services in August 2008 to 22.4\% in June 2010 (KIHASA 2010).

In this regard, the LTCI expanded the responsible family member of the recipients. Firstly, there came into force stricter regulations on the scope of the obliged family member of the recipient. According to notification No. 2009-125 of the MHW of 30 June 2009, the definition of cohabitation was extended from living in one house to live in the same building, which meant the responsibilities for elderly care partly moved from the state to the family.

Secondly, the level of compensation for family care decreased. The recognised hours for in-home care services offered by the cohabited FCH decreased from 120 minutes per day to 90 minutes per day, and when a recipient received in-home care services from the FCH, the recipient was not allowed to use additional in-home care services on the same day. Also, 
the regulation noted that the compensation for home visit care services offered by the cohabited FCH covered only physical support for the recipients.

These attempts by the administration can be understood as a response to the radical increase in the FCHs' caregiving (Lee Yun-Kyung 2010: 99). Although the administration considered how family caregiving should be arranged in the new social LTCI programme, it was only considered in the context of financial constraints and the growth of social service industries as a social investment. Despite the strict regulations of the administration, the FCHs' caregiving had constantly increased. NHIS (2012) reported that the number of claims for in-home care services offered by a cohabited FCH increased from 16,509 (16\% of the total claims) in September 2009 to 32,015 in March 2010 (NHIS 2012, in Yang NanJoo 2013).

\section{Reform of payments for FCHs in 2011}

Between June 2008 and June 2010, the notifications on the payments for the family caregiving had moved partly into the family’s responsibility. However, there was no critical regulation on the status of the FCH until 2011.

In June 2011, the administration announced a notification regarding the amendment of the compensations for home visit care services offered by a FCH. The notification No. 201172 of the MHW contained three important amendments regarding the payments for family caregiving and the status of the FCHs.

Firstly, the scope of the definition of a family member who has the primary care responsibility for the elderly was changed. The previous concept of the family member who had the primary care responsibility for the recipient of the LTCI was cohabited family members according to Civil Code Article 779 (see footnote 7), so that the cohabited FCHs got reduced recognition hours (90 minutes per day) for their home visit care services for their family member who was entitled to the LTCI. However, the scope of the definition of a family member who had the primary care responsibility for the recipient was extended to all family members. All the family members, according to Civil Code Article 779, regardless of living arrangements, have the primary care responsibility for their family member. Because of this regulation, there was no need for the division of FCHs according to living arrangements. 
Secondly, the notification regulated the level of payments. Regardless of the living arrangements, the recognised hours for home visit care services for FCHs decreased from 90 minutes per day to 60 minutes per day, and only 20 days per month. However, the administration contained an exemption for those who are over 65 years old and for a spouse of a recipient of the LTCI, or those who care for recipients who suffer dementia with a disposition towards violence.

Lastly, in addition to reductions in the number of recognised hours, working conditions and social rights to pensions and health insurance were restricted since the FCHs are in family relations with the recipients, and this could be considered as an untypical status in the LTCI system. The care centre does not have to ensure working conditions for the care helpers, such as severance money, employer's share of contributions to the National Pension System and the NHI, as well as payments under the public unemployment insurance programme and occupational accidents compensation.

The changes in the payments for home visit care services offered by FCHs can be summarised regarding three aspects of the status of the FCHs, as follows:

Table 2.4. Changes in the status of FCHs compared to non-familial care helpers

\begin{tabular}{|c|c|c|c|c|c|c|c|c|}
\hline & \multicolumn{3}{|c|}{2008} & \multicolumn{3}{|c|}{2009} & \multicolumn{2}{|c|}{ After July 2011} \\
\hline $\begin{array}{l}\text { The scope of } \\
\text { primary } \\
\text { obligated family } \\
\text { member }\end{array}$ & \multicolumn{3}{|c|}{$\begin{array}{l}\text { Cohabited family } \\
\text { member }\end{array}$} & \multicolumn{3}{|c|}{$\begin{array}{l}\text { Cohabited family } \\
\text { member }\end{array}$} & $\begin{array}{l}\text { All family } \\
\text { Civil Cod } \\
\text { irrespectiv } \\
\text { arrangeme }\end{array}$ & $\begin{array}{l}\text { ers according to } \\
\text { le } 779 \text {, } \\
\text { ving }\end{array}$ \\
\hline \multirow{2}{*}{$\begin{array}{l}\text { Level of } \\
\text { payments } \\
\text { (recognition } \\
\text { minutes) }\end{array}$} & $\mathrm{N}^{1)}$ & $\mathrm{FS}^{2)}$ & $\mathrm{FC}^{3)}$ & $\mathrm{N}$ & FS & $\mathrm{FC}$ & NF & $\mathrm{FS}$ and $\mathrm{FC}$ \\
\hline & 240 & 240 & 120 & 240 & 240 & 90 & 240 & $\begin{array}{c}\text { 60, } 20 \text { days (90, } \\
\left.30 \text { days }^{4)}\right)\end{array}$ \\
\hline $\begin{array}{l}\text { Restriction on } \\
\text { extra working } \\
\text { hours }\end{array}$ & \multicolumn{3}{|c|}{ No limitation } & \multicolumn{3}{|c|}{ No limitation } & \multicolumn{2}{|c|}{$\begin{array}{l}\text { Only up to } 18 \text { hours per week } \\
\text { extra work as an employee } \\
\text { besides FCH service provision. }\end{array}$} \\
\hline
\end{tabular}

Notes: 1) Non-familial care helper, 2) FCH with a separate living arrangement, 3) FCH cohabited with the recipients, 4) exceptions for recipients' spouses aged 65 and more as well as FCHs who care for a recipient suffering dementia with a disposition towards violence.

\subsection{Conclusion}

This chapter overviewed the living status of the elderly and their family relations regarding care practices in Korea. Old age is the stage of life where people are confronted with various challenges, like the weakening of economic power and physical ability as well as changes in family relations. The traditional practices of intergenerational financial and care supports 
have been changing. Although public income security for elderly people has been gradually established, the present elderly are in a blind spot of the system, since they did not have enough time to contribute to the NPS in their working life. Against this background, the Roh Moo-Hyun government tried to cover the shortage of the present elderly's income by introducing the basic pensions benefit. However, most of the elderly households still rely on private transfers from their adult children. Those who have no possibilities of either public or private transfers have to be willing to work for a living in their old age. Indeed, the employment rate in old age, meaning people with 65 years and over, in Korea is relatively high compared to other OECD countries.

Besides the income source, the living arrangements and personal care for the elderly, too, have undergone significant changes over the last years. The cohabitation rate of the elderly with adult children has diminished in not only the rural areas but also in urban areas. As the average life expectancy has been rising and the overall population has been ageing, concern has been voiced about the health status of the elderly, especially regarding complex diseases and increasing medical expenditures for them. To answer to increase expenditures on long-term care, the government introduced the LTCI for the elderly in 2008.

The change in cohabitation models has an impact on personal care as well as family relations. With regard to family relations, although Korean society looks as if firmly constructed on the basis of the moral value 'hyo (효, 孝)', this traditional care culture has been increasingly losing importance. This and underdeveloped social care services have led to most of the present elderly in the country facing a number of difficulties in their later life. However, even in Western welfare societies, family caregiving for the elderly has not been completely substituted by public elderly care provisions.

The previous section 2.4 has described the peculiar status of the FCHs in the LTCI for the elderly in Korea by explaining the service delivery system in the LTCI and tracing the trajectories of changes in compensation for LTC services offered by FCHs. The case of FCHs in Korea shows multi-faceted characteristics of care in relation to the welfare state and helps to overcome dichotomies about the concept of a family caregiver, such as formal/informal, qualified/unqualified, and paid/unpaid. FCHs can be considered neither as wage workers nor as pure family members due to their complicated situation regarding the payment system for their care and the restrictions on extra work in the sense of employment in the paid labour market. 
The trajectories of changes in the payments to FCHs reveal how the welfare state arranges and regulates the public provision of social services. The FCH programme in the LTCI can be understood as following a trajectory of compromises regarding the responsibilities for elderly care between state and family within the long-term cultural legacy of Korean family-centred values. The elderly care programme in Korea aimed to socialise elderly care with the introduction of the LTCI. The responsibilities for the elderly care, however, have moved from the state towards the family. With these compromises, it became more difficult for FCHs to obtain social rights to pension benefits and individual entitlements as economic participants.

To summarize, this chapter gave the local context of how the current changes in later life and family relationships in Korea would affect family care for the present elderly. Especially, the trajectories of the changes in the payments for the FCHs provided the analytical angle on how the Korean welfare state compromises the care responsibility with family at a collective level. Although the level of payments for the FCHs could be an indicator for limited compensation and imperfect social rights in elderly care, there is yet no evidence on how the FCHs experience caring for their old family members. Specifically, there is need to investigate the process of becoming and being a FCH, which enables us to get insight into how positive and negative factors in caring for the elderly are interwoven throughout everyday experiences and how the traditional care culture in Korea affects the care responsibility among actors at two different levels (macro and micro). The empirical findings of this study will be presented in Chapter V (Results). Before showing the results, however, I will propose conceptual aspects and discuss the analytic framework for my study in the next chapter (III). 


\section{CHAPTER III LITERATURE REVIEW : Sharing Care Responsibilities at Two Levels 3.1. Introduction}

Based on a literature review, this chapter aims to build an analytic framework to investigate the experiences of family care helpers (FCHs, gajokyoyangbohosa), who are family caregivers with a licence for a care helper ( $\mathrm{CH}$, yoyangbohosa). This study uses the concept of 'care responsibility' as a key term to analyse elderly care practices among family members. As care responsibility is the central concept that will be used to explore the experiences of FCHs, this section reviews how care responsibility is conceptualised in the literature - including the characteristics and common elements of the concept of responsibility - and it focuses on cultural contexts concerning care practices among family members.

This chapter presents the approaches used to define the term 'care responsibility', and the theories and perspectives used to comprehend it, both from a macro- and a micro-level perspective. In the context of care responsibility, the macro-level perspective enables us to understand how care responsibilities are arranged among actors in a society, for example, the state, the market and the family. Besides, the micro-level perspective helps us to understand elderly care practices among family members, focusing on the experience and the nature of family care practice.

This framework supports the main research questions and purposes of this study on the experiences of family care helpers (FCHs) related to caring for the elderly in the specific context of an East Asian developing country, i.e. Korea. The study of the experiences of the FCHs through the lens of a micro-perspective will enable us to understand how the care responsibility is shared among family members and, in the course of this, how the formal long-term care program for the elderly in Korea affects the FCHs' perceptions of their caregiving for the elderly. A significant part of the literature used in this framework is specific to the European context, where much of the research on elderly care has been carried out, as research on elderly care had not been well developed in Korea, except for research on elderly care from the perspective of familial behaviour in traditional care practices. However, the studies on care as a form of social risk have emerged since the 2000s, when the population has been ageing and family structures and attitudes towards 
elderly care in a family setting in Korea have been changing. As will be developed in this chapter, this study advances knowledge on elderly care in Korea to contribute to the understanding of this phenomenon in new ageing societies where this topic remains unstudied.

This chapter begins by describing the concept of responsibility. In the following sections 2.3 and 2.4, I review care both from macro- and from micro-perspectives. In section 2.3, I review how the responsibility for elderly care is arranged among the family, the market and the state, and section 2.4 explains how family care practice works between two individuals and among family members.

\subsection{Care Responsibility, Social Rights, and Social Care}

This section reviews the concepts that will help us to understand elderly care by the FCH. I apply the concepts of 'care responsibility', 'social rights' and 'social care' to build an analytical framework for this study.

\subsubsection{Concept of care responsibility}

The concept of responsibility refers both to a set of duties and tasks and to accountability. What kinds of property does the concept of responsibility include? What is the nature of responsibility? Is it a matter of ethics, or emotion, or of relationships based on love and affection, or of tasks, labour and resources?

To understand the concept of responsibility is essential for this study due to its multifaceted and complex nature. Tronto and Fisher (1990) have identified four phases of care as a framework for analysing these relations, particular caring work with its ethical dimensions. The four phases of care are 'caring about', 'caring for', 'caregiving' and 'care receiving'. In addition, four moral elements of care are identified as the moral dimensions of the four phases of care, respectively: attentiveness, responsibility, competence and responsiveness. The notion of responsibility as a moral concept is related to the phase of 'caring for'. Tronto (2001) explains how the phase 'caring for' is linked to the moral dimension of 'responsibility':

'Caring for' is the phase in caring when someone assumes responsibility for meeting a need that has been identified. Simply seeing a need for care is not enough to make it to happen; someone has to assume the responsibility for organizing, marshalling resources or personnel, and paying for the care work 
that will meet the identified needs. The moral dimension of 'caring for' is to assume responsibility and to take it seriously. (Tronto 2001: 63)

In identifying these phases of care, Tronto (2001) suggests some questions regarding the responsibility and the phase of caring for: Who should be responsible for meeting the needs for care that do exist? How can and should such responsibility be fixed? How distant from those who give care are those who hold 'responsibility'? The words 'who' in Tronto's questions can be interpreted as two different levels: one is who are the actors in a society like the state, the market, and the family at a collective level, the other is who is the specific person who takes on the care responsibility in practice.

Care is comprised both of a relationship and a set of tasks. Therefore, with the concept of care responsibility one can consider care from the perspective both of the tasks and of the emotional responses that are involved. Elderly care is a practice based on relationships and emotions (Kim Hye-Kyung and Namgung Myung-Hee 2009; Kim Kyounghee and Kang Eun-ae 2010; Park Ki-Nam 2013). Kim Kyounghee and Kang Eun-Ae (2010) point out that care is related to responsibility for the relational nature of care. Care is not mere labour and carrying out a set of tasks but is a situation where emotions and relationships interact between a care receiver and a caregiver. Therefore, the nature of care can only be captured by considering these relationships.

Although all kinds of care are based on a relationship between a care receiver and a caregiver, family care is regarded as the most relationship-driven care form (Kim HyeKyung and Namgung Myoung-Hee 2009; Kim Young-Ock and Kim Young-Sook 2011; Namgung Myoung-Hee 2009; Park Ki-Nam 2013; Yun Seong-Hee 2012). Family care is based on family relationships which also affect the relationships that exist during and after caring for members of their family. In many cases, a caregiver is likely to cohabit with her care receiver and this also leads to deep interactions between two people. There have been studies on care responsibility from the standpoint of family obligations based on normative moral guidelines towards the caring state, which gives priority to care in society (Lewis and Meredith 1988; Namgung Myoung-Hee 2010; Seo In-Soon 2003). While obligation is a notion that is based on moral values among individuals, the concept of responsibility has a broader sense.

While care is a matter of emotions and of ethics, it requires certain conditions for it to take place in reality. In other words, the nature of relationships and emotions is the internal drive 
for the exercising of care responsibility, and it is completed through the corresponding behaviour and activities which require time and resources. The fact that care responsibility has both the aspects of relationships based on normative guidelines and tasks required to achieve results leads to a divergence between the perception and the reality of elderly care provision. Shin Kyung-Ah (2011) points out that the aged in Korea prefer for normative reasons to be cared for by members of their family, but they also acknowledge that the family cannot take the responsibility for elderly care alone in reality.

Care responsibility can be looked at from various perspectives. It is found among individuals, the family, society and the state and it can be altered by social changes. As more women participate in the labour market and as society is ageing, the family members cannot care for their family alone anymore, but various actors in a society must share the care responsibility. There have been a number of studies on the responsibility mix for care (Hwang Jung-Mi 2007).

Firstly, care responsibility may exist between two individuals. Care is a relational practice between two individuals, a dependent one and an independent one. The situation of need for care arises from the existence of the one who is dependent. However, this approach in terms of dependent/ independent individuals has a weak point in that the dependent one always remains vulnerable and the independent one is likely to feel excessive stress in the relationship of caregiver and care receiver (Hwang Jung-Mee 2007: 45; Lee Hyung-Joo 2006; Seo Insoon 2003).

However, care responsibility works not only between two individuals but also at the family level. Finch (1989) has categorised the family relations which support each other. The support for the family occurs in various family relations such as parents and children, grandparents and children, relatives-in-law and brothers and sisters. They support each other in providing various kinds of help, including economic support, accommodation, personal care, practical care and emotional and moral care. Family care is based on family obligations which are a matter of people's moral responsibility for their family. Therefore, the care responsibility for the elderly among family members has to be differently understood from the responsibility of those outside the family.

Meanwhile, care responsibility is constructed on a collective level. Responsibility for the elderly has become one of the social issues in that elderly care is not merely one form of 
dependent relationship but is a typical stage, which everyone experiences at least once in their life. In this sense, not only participating in the paid labour market as an individual worker but also caring for one's family at home should be recognised as a requirement of getting full citizenship in society.

\subsubsection{Social rights and social care}

Drawing on Marshall (1950), the concept of 'social rights' are generally been defined as "a person's rights to demand conditions that allow for a life of human dignity, a term alternatively known as the fundamental right to a decent life". It originated from the concept that the security of the lives of the populace is the responsibility of the state. Social rights can be multi-dimensionally defined and categorised into various branches. The idea of social rights is based on a social policy that started in the western welfare state in the middle of the 20th century. Marshall (1950), who theorised the concept for the first time, argued that "citizenship is a set of rights that have been gradually developed from civil rights to social rights". He refers to the transition 'from the right to a modicum of economic welfare and security to the right to share to the full in the social heritage and to live the life of a civilised being according to the standards prevailing in the society' (Marshall, 1950: 11). Marshall's concept of social citizenship provided a theoretical basis for the expansions of public welfare that took place in the Western welfare states after World War II. As Hobson and Lister (2002) have argued, Marshall's concept of social rights was premised on the western welfare states in the post-World War II period and it has focused on the intervention of the state, such as in income security. Therefore, new social rights should expand into participation in the public area, with respect and acceptance from others (Hobson and Lister 2002; Matthews and Erickson 2005). The expansion of social rights does not mean the same thing to everyone. The acquisition of social rights has followed a different pattern, depending on social groups and historical backgrounds (Walby 1994). For minorities, the characteristics of citizenship are not universality and inclusiveness, but selectivity and asymmetry.

The debates on social rights in the context of FCHs are significant. The limitations of the FCHs' social rights are caused by the limitations of the conventional concept of social rights; the conventional framework cannot bear the FCHs' social rights for two reasons. Firstly, because the FCHs do not have conventional standard employment relationships, they cannot obtain employment rights, such as workers' compensations, as well as pensions 
and unemployment benefits. However, even if they enter into a contract to provide the service of elderly care, they cannot obtain social rights. On this point, the second reason arises: social rights are fundamentally based on the principle of maintenance, in that they aim to provide against the risk of the dominance of the male breadwinner, with wives and children regarded as dependents (Sainsbury 1996: 46). Concerning this matter, there are two approaches to consider: one is an improvement of women's economic dependency (Hobson 1990). However, this is likely to lead to 'the contemporary variant of Wollstonecraft's dilemma' (Lister 1994, cited in Knijn and Kremer 1997: 350; Razavi 2007). This is because, even though some women may achieve parity in the labour market, care and domestic work do not disappear but pass onto others, and this intensifies the gap between women in terms of social class. The other is the recognition of qualifications and the principle of care (Sainsbury 1996: 46).

Knijn and Kremer (1997) also advocate expanding the idea of citizenship to the rights to care and be cared for to help solve the dilemmas of care. Kremer (2007) has suggested the concept of rights to give care as compensations for caregiving such as tax, social security and leave. Besides these compensations, cash payments for care are one of the ways of compensating for family caregiving.

Meanwhile, I will apply the concept of 'social care' to capture the social nature of care. Elderly care has become one of the most important subjects in contemporary welfare states. This is not only because of demographic changes like increasing life expectancy and low fertility but also because of social changes like increasing female economic participation and changing norms towards caring for the elderly. Due to these changes, the point of view on elderly care should move from the domestic area to social issues which require collective solutions (Ma 2011; Moen and DePasquale 2017). Daly and Lewis (2000) argue that care has some social characteristics and they suggest using the concept of 'social care'. They highlight the social nature of care, which has three different aspects: i) care as labour ii) care as an obligation and a responsibility iii) care as activities with financial and emotional costs. The concept of social care helps us to understand better how welfare states construct funds and provisions for elderly care. (Daly and Lewis 1998;2000; Kröger 2001; Peng 2002;2010;2011;2014; Anttonen et al 2003)

Engster (2007) claims that old welfare states cannot deal with the new risks caused by social changes and he maintains that new welfare states are needed. He suggests the term 
the 'caring state' which is based on three conditions. Firstly, the 'caring state' see needs for care as normal, since one is born to be dependent on other beings and one needs care at least once in one's life. Therefore, dependency is a universal experience in one's life course. The care state is based on the care relationship, which takes priority over relationships in the market and in politics. Lastly, welfare is based on the collective responsibility of the whole society. The state should support individuals in caring for their family through policies and institutions.

I assume that the social rights of FCHs are limited because they cannot acquire them under the condition of conventional social norms; the social status of FCHs does not apply within the conventional framework of social rights because they are beyond both the publicprivate distinction and the paid-unpaid distinction ${ }^{8}$. Concerning the status of FCHs as carers, the social rights of the FCHs are classified under four headings: The right to have access to paid work; the right to not necessarily have to take paid work (decommodification), the right not to be required to take unpaid work (defamiliarization), and the right to both give and receive care.

To conclude, the concept of care responsibility enables us to comprehend the nature of care, especially family care, in that this responsibility includes various facets, including relationships, labour, resources and morals. Responsibility plays a pivotal role in family care. Moreover, the concept of responsibility can be applied at multiple levels, from individual to collective levels. In these senses, some questions arise, such as: How does the state arrange the elderly care responsibility among various actors in society? How does the family's role change in sharing care responsibility? How does the family experience in caring for the elderly? Has the family's responsibility for elderly care decreased or increased?

The following sections of this chapter discuss more details of the macro- and microdimensions of care responsibility, focusing on i) sharing the care responsibilities among various actors in society, social recognition for the care offered by family members and how the care culture has an impact on the care arrangements in a welfare state (macro-level analysis); and ii) A literature review on the experience of family caregivers in terms of

\footnotetext{
${ }^{8}$ To better understand the characteristics and reasons of the deficit of FCHs' social rights, section 2.4. in this study explores how state of the art has influenced the general population's concepts of social rights, from the perspectives of international standards, the constitution and social policy.
} 
ideals of care, care burdens and stress in relationships with the elderly and with other family members in daily life (micro-level analysis).

\subsection{Care Arrangements}

As was mentioned above, care responsibility is a relationship entailing a care receiver and a caregiver. At the same time, the care responsibility contains accountability for performing or guaranteeing some behaviours and actions which entail labour and resources. Care responsibility is also a moral value and it is a matter of normative guidelines, which could be captured by the care arrangements. This section explores how the family organizes the care arrangements in a welfare state at a macro-level. Based on the three concepts of 'care responsibility', 'social rights', and 'social care' discussed in the previous section 3.2, section 3.3.1 reviews the theories and the literature on how elderly care is arranged among social actors in welfare states and where the family fits into the arrangements. Specifically, section 3.3.2 explains the debates on cash for care. A literature review on how care culture influences institutions in the welfare states will be also given in section 3.3.3. Based on the literature review on care arrangements, cash for care and the impacts on the care culture, I consider the contested position of the family care helpers (FCHs).

\subsubsection{Care arrangements}

Elderly care has traditionally been provided mainly at home by family caregivers, but traditional elderly care by family members may not be sustainable anymore. Instead, society and the state should share the responsibility for providing care collectively (Hwang Jung-Mee 2007; Kim Ju-Hyun 2016). Glenn (2010) discusses the social organisation of caring, which can involve systematic ways of ensuring the distribution of responsibility for care work. The care organisation is a mixture of paid/ unpaid, private/ public elements and it changes according to time and place.

The concept of care arrangements refers to the configuration of the sharing of care burdens among actors in society, such as the family, the market, non-governmental organisations and the state. With respect to cost-sharing, there are also studies on how to arrange the costs for elderly care (Theobald 2012). Based on the concept of 'social rights' and 'social care', there are various studies on care arrangements, which show how the tasks and responsibilities for care are divided among the state, the market and the family (PfauEffinger 2005). There are studies on how the state in Korea intervenes on care (Kwon Soo- 
Hyun 2013). The care arrangements can vary according to the context of the society. Several scholars have discussed how care arrangements can be adequately captured and they have suggested some models for care arrangements (An and Peng 2015; Razavie 2007). An and Peng (2015) suggest three different provision-finance nexuses as an analytical framework for welfare states and care arrangements. They argue that welfare states may be divided into three different nexuses according to their proportional shares of the provision-finance nexus: publicly financed and provided, publicly financed and privately provided, and privately financed and provided. According to which value is more focused on by welfare states, the compensation types for family caregiving can be categorised into two: valuing care through time, such as leave provision, and valuing care through money, such as an allowance (An Mi-Young 2017: 187). In this case, welfare states play a pivotal role in the configuration of care systems and arrangements by using legalisation and amendments based on a state's welfare vision and family values (PfauEffinger 2005b: 327ff.).

Politics and the state play an essential role in legalisation and in amending policies on care arrangements. Estévez-Abe and Yeong-Soon Kim (2014) reported that Korea was known for having family-centred care arrangements but, in recent years, childcare has increased as a consequence of the political compromises between political parties and interest groups.

Scholars acknowledge that the traditional family system in Korea is not sustainable as a way to protect and care for individuals in society. Instead, an alternative to care for individuals socially and collectively is needed (Kim Ju-Hyun 2016). Chu Yousun (2011) argues that, for a long time, care was not a public issue in South Korea. Instead, it was only performed in relationships by women in the domestic arena without pay. However, later, due to women's increasing economic participation and the ageing population, Korean society asked for research and policies on care, so that the notion became accepted as part of public and formal issues. The long-term care insurance (LTCI) for the elderly in Korea was implemented in the context of the state's responsibility for elderly care and ensuring social rights to receive elderly care (Shin Kyung-Ah 2011: 70).

Since the 2000s, the responsibility mix of care has become one of the most significant issues in Korean society. Sohn Yong Jin (2010), in his account of the Korean babyboomers' perceptions on care responsibility for their parents, pointed out that adult children who feel more associational and affectional intimacy with their elderly parents perceive the 
responsibility for elderly care as the family's responsibility rather than the collective responsibility of society. He maintains that the family's responsibility for elderly care is not a trade-off for collective responsibility, so the care responsibility could be shared among various actors in society.

Hwang Jung-Mee (2007) argues that issues relating to the notion of 'responsibility mix' have not been sufficiently discussed given that the elderly care deficits have increased in Korean society where both men and women have to enter the paid labour market. Notably, it is necessary for society to discuss how the responsibility for care should be shared, involving both the state and the family; how men and women can share the responsibility for care in the family; how families can choose to use different types and sets of elderly care arrangements (Hwang Jung-Mee 2007: 35). The notion of a responsibility mix is as important as that of a welfare mix, which is a discussion of how the different kinds of welfare programmes and services could be harmonized in different settings and arrangements, including the state, the market and the family (Hwang Jung-Mee 2007: 45).

In terms of financing for elderly care, there are various approaches based on cost and social capital. Expenditure on care is the cost to society that has to be reduced. On the other hand, from the perspective of social capital, public care provision enables us to investigate greater human resource development.

When formal elderly care is provided, how does informal family care change? As a new formal setting for elderly care is implemented, the formal setting is likely to substitute for the former informal family care. Alternatively, will family caregiving still remain and will formal care play only an additional role in elderly care? There are contradictory positions in the debates on the effects of the LTCI. In general, there is research that argues that formal elderly care provision is likely to substitute for family caregiving in informal settings (Kim Young-Ock and Kim Young-Sook 2011; Ham Sunyu and Hong Bageui 2017). Ham Sunyu and Hong Bageui (2017) maintain that formal care does not supplement the former informal care but substitutes for informal care.

\subsubsection{Debates on cash for care}

As was shown in the previous section, the family plays an essential role in elderly care, even if new formal care provisions have expanded. In arranging elderly care services, the state finds ways to recognise and compensate for the elderly care offered by the family 
Indeed, the state takes a variety of measures to recognise family care, for example, paid care leave, pension credits and care allowances. In Korea, taking care leave to look after frail elderly people is not yet widespread, but some larger companies allow their employees to take leave to care for the employee's frail parents. Kremer (2007) suggests the concept of 'rights to give care' as compensations for caregiving such as tax, social security and leave. Apart from these compensations, cash payments for care are one of the ways of compensating for family caregiving. Ungerson (2000: 71) has categorised five different ways of recognising family care socially:

1) Caregiver allowances paid through social security and tax systems.

2) Proper wages paid by the state or state agencies.

3) Symbolic payments paid by care users to kin, neighbours and friends.

4) Paid volunteering paid by voluntary organisations and local authorities to volunteers.

5) Routed wages paid via direct payments to care users.

However, in the case of the family caregivers, the care recognition for them is hard to define.

For example, Pijl and Ramakers (2007) paid family caregivers who were led to think of themselves as workers. Then, the question arises how to recognise those who care for their family at home instead of working in the paid labour market? How can their social rights be insured? Usually, they are entitled to pension benefits and social services as supplementary recipients of the male breadwinner who works in the paid labour market.

While there have been many arguments and discussions regarding payments for elderly care services in the western countries, there have been fewer tendencies towards cash payments for elderly care in Asian countries due to the old care traditions and an undeveloped welfare system. Seok Jae-Eun (2006) noted that the government was not allowed to provide cash for elderly care in the discussion of the introduction of the public long-term care (LTC) system in Korea, since the government worried about payment abuses and that cash for care could lead former informal caregivers, who were mainly women, to stay at home instead of entering the paid labour market. However, Lee YunKyung (2010) reported that, as the number of FCHs had increased, so has the number of 
claims for cash payments ${ }^{9}$. Although the payments for FCHs differ from cash for family care, such as care allowance or attendant allowance, the payments that the FCHs received are likely to be perceived as cash payments. She asserts that there needs to measure such as cash payments to respond to the needs of the recipients and the family caregivers. In contrast, Hong Sung Wook (2011) asserted that the government should remain committed to the principles of in-kind benefits in the LTCI and the government should improve the quality of care by introducing a care management system.

Nevertheless, the recognition of the value of FCHs' caregiving does not in itself guarantee social rights. These debates reveal how FCHs' social rights are represented by forms and levels of payments for care. Ungerson (2005) discusses the moral and political issues arising from the payments for care. Since they have a special relationship with those cared for, the different kinds of rights can conflict concerning the types of provision and support for care: the carer's autonomy versus the elderly's privacy, and attendance allowances versus care allowances (Ungerson 2005: 43ff).

\subsubsection{Care culture and the welfare state}

Engaging a family care helper (FCH) in the LTCI in Korea is also understood as a response of family caregivers from the perspective of the care culture. Pfau-Effinger (2005a) introduced the concept of 'welfare culture' in the sense of welfare state analysis, which means 'the relevant ideas in a given society surrounding the welfare state and the way it is embedded in society’ (Pfau-Effinger 2005a: 4). Specifically, although the same welfare programmes may be introduced, the effects and consequences will vary according to the state's cultural context (Pfau-Effinger 2005a: 7).

\footnotetext{
${ }^{9}$ In the LTCI in Korea, the cash payments are minimal. The cash payments are allowed only in cases according to Article 24 of the LTCI for the elderly as follows:

Article 24 (Family Care Cash Benefits) (1) If any of the following beneficiaries have received long-term care benefits equivalent to home visit care under Article 23 (1) 1 (a) from a family member or another person, the NHIS may provide the beneficiary with family care cash benefits in accordance with guidelines prescribed by Presidential Decree:

A person who resides in an area determined and publicly notified by the Minister of Health and Welfare as an area in which long-term care institutions are significantly insufficient, such as an island or a remote area;

A person recognized by the Minister of Health and Welfare as one who has difficulty in using long-term benefits provided by long-term care institutions due to a natural disaster or any other reason similar to it;

A person who needs to receive long-term care from his/her family members. Due to his/her physical or mental conditions, character.

(2) Procedures for reimbursement of family care cash benefits under paragraph (1) and other necessary matters shall be prescribed by Ordinance of the Ministry of Health and Welfare.
} 
In Asian countries, the traditional care culture for the elderly was mainly based on filial piety in the sense of Confucianism, so the previous studies on elderly care had also been about the experiences of family carers as informal carers in domestic areas. During the period of the introduction of the LTCI, there were studies on how the LTCI would affect the family (Chang Kyung-Sup et al. 2015; Choi In-Hee et al. 2011; Ham Inhee 2014; Ham Sunyu and Hong Bageui 2017; Kong Seonhee 2013; Lee Jaerim 2015; Sohn, Yong-Jin 2012). However, as it was only a short time since the formalisation of elderly care had become universal in Korea, there has been little research into the complicated and confused situation relating to family caregivers after the introduction of the LTCI (Choi In-Hee and Kim Jung-Hyun 2013; Lee Min-Sook 2012; Yang Nan-Joo 2013).

Chang Kyung-Sup et al. (2015) argued that the idea of familialism in Korea differs from that in the European context. The concept of familialism in the European context refers to the fact that welfare states try to protect through their social policies and institutions the conservative family model, which is a male breadwinner and women carers, and the state has made the family responsible for welfare provision. Although the Korean context of familialism is also family-centred, as in the European context, in the past, care could be divided into two kinds: unpaid informal care mainly offered by family members and paid care offered by wage workers in the labour market. However, demographic and social changes are blurring the boundaries between formal and informal as well as paid and unpaid care. Frericks et al. (2014), in the analysis of the family care policies of three European counties, argued that the level of the formalisation of care varies considerably, from semiformal to formal, classified by family care regimes. However, it is hard to decide in some cases whether the elderly care programme is family-centred. Kodate and Timonnen (2017) discussed new trends in the welfare states where the elderly programme seems defamilialised but, in reality, the state has re-introduced family care stealthily through the back door. Lee Min-Sook (2012) also reported that FCHs in Korea have experienced confusion in recognising the boundaries between caring for family members as being the job of FCHs, who have obtained a care helper licence, and as part of daily life which is related to family obligations and love.

Family care is not only an element of family policy but is also a practice which is intertwined with emotions and relationships between persons. Therefore, it is essential to explore family care practice at the micro-level. 


\subsection{Family Care as Family Practice}

The previous section 3.3 showed how the FCH programme in the LTCI in Korea is closer to 'familialism' than to ‘de-familialism'. It relies heavily on families, and more specifically on women, to offer elderly care. This section explores how family care practices are constructed and from what elements and how these affect changes in family care practices. To answer these questions, I will review the previous literature on the theory and concepts which have contributed to comprehending the characteristics and properties of family care and then I will analyse the different approaches to study the care experience. This will provide the analytical framework to study care from a micro-level perspective.

To understand the concept of care responsibility at a micro-level, I suggest the concept of 'family care practices', which is a specific form of 'family practices' (Morgan 1996; 1999). Drawing on Morgan (1996; 1999), the concept of 'family practices' focuses on 'the everyday relationships and activities of families, which is something that individuals do with family matters' (Morgan 1996: 192). As was mentioned in Section 3.2, care is a set of tasks and relationships at the same time, and individuals in family caregiving are assumed to be relationship-oriented. Care practices are matters of relations between caregivers and care receivers. In caring, the interaction between two individuals is essential: in the case of family care, it is a matter of the family relationship and history. In addition, family caregiving also matters to other family members in the context of family values and social norms.

Family values are the foundations of justification for care for elderly family members. Elderly care has been understood as one of the important family practices that show one's pursuing of family values. Family values and norms are captured in the form of the ideals of care in a certain society. There is a prevailing ideal of elderly care in a society, which contains the context and the social norms that exist in a society. In Korea, since elderly care by family members at home has been considered as a fulfilment of children's responsibility in the sense of family obligations. Indeed, studies on Korean elderly care trends have reported that although people can afford quality elderly care services outside the family, the elderly and the family do not want to use these services, since the ideal of care in Korea is still to be cared for by family members at home (Cho, Myoung-Ok 1999; Choi In-Hee et al. 2013; Lee Dongok 2011; Lee Min-Sook 2012; Lim Byungwoo and Park Sungyul 1999; Yang Nan-Joo 2013). This attitude differs from the European concept of 'ageing in place', 
which means that elderly people stay in their place and keep their lifestyle after they get frail. In contrast, the elderly in Korea accept moving from rural areas to big cities when they get frail (Lee Hyunsook 2011). Even if they have to accept abrupt changes in their lifestyle, they choose to live together with their adult children and to be cared for by them. ${ }^{10}$

In Korea, most studies about care responsibility at the family level are about the traditional normative guidelines of children's obligations towards their parents. Lee Duck Sik (2015) maintains that a son's obligations are related to human rights and the rights of life, and the traditional family values of the son's obligations should be widespread due to the recovery of the family relationship. However, his approach overlooked the hierarchy that exists between family members. In some cases, care for the elderly could be burdensome for other family members. Especially, scholars acknowledge that family responsibility for elderly care has been dominant as an ideal of elderly care in Korea (Hwang Jung-Mee 2007; Song Da-Young 2003)

The responsibility for care is not equally divided among family members. In the past, the responsibility for family care lay on the shoulders of the women at home (Kong ByungHye 2010). Recently, the responsibility for family care has been shared and negotiated among family members. There have been a series of studies on daughters-in-law who experience a heavy feeling of responsibility for elderly care (Cho Hye-Young et al. 2015). Family caregiving is sometimes more challenging when compared to non-familial caregiving relationships since duty takes over in caring instead of love and the moral obligation to uphold caregiving leads to caregivers' sacrifice (Finch 1989; Komter 2004). Cho Hye-Young et al. (2015) have pointed out that although formal elderly care provision has expanded in Korea, families do not use the formal elderly care services due to the old parents' negative perceptions of the formal elderly care services, and the daughters-in-law feel a responsibility to meet their parents-in-law's needs for care in the sense of family obligations.

Although family-centred care as the ideal of care has a significant impact on family care practices, the family values take the form of normative guidelines rather than precise descriptions. This means that the values differ from how an individual behaves in reality. And the guidelines would be changeable as a society changes in its family structure and

\footnotetext{
${ }^{10}$ See more details in Section 2.3.1.
} 
care culture. In her analysis of elderly care by adult children, Namgung Myoung-Hee (2010) suggests the concept of 'relational autonomy' in care practice and negotiation of the care for elderly parents as an alternative ideal of relationships in intergenerational caregiving and as a strategy for coping with the traditional expectations of family and society in caring for the old parents. Given the hierarchical orders between the elderly and the adult children based on the care, the children are likely to feel more stress and they cannot bear the care responsibility anymore in a changing modern society. In this regard, a question arises: given these social changes and the implementation of the LTCI, how do the family's perceptions of the ideal of elderly care change?

Apart from the care burdens caused by relationships, care as a set of tasks has an impact on the caregivers' well-being. As non-familial caregivers' informal settings, family caregivers dedicate their time, energy and resources to their care for family members. Even if the relationship between family members is in a good condition and family caregivers are willing to care for the elderly, the family caregivers experience various challenges in their daily life. The burdens of family caregivers are one of the issues that are most focused upon in elderly care, and there have been various studies on the caregivers' burdens (Lewis and Meredith 1988; Finch 1989; Komter 2004).

Families feel stressed and burdened with caring for the elderly at home, but such care also has a positive influence on the caregivers' quality of life. Family caregivers experience a recovery in their relationship with the elderly and they feel proud that they can care for the family members by themselves (Kang Tae-Wha et al. 2014; Lee Min-Sook 2012; Namgung Myoung-Hee 2010). Nevertheless, it is hard to separate the positives from the negatives in family care. Rather, both the positives and the negatives are likely to be interwoven in the process of family care. And the experiences of family care vary according to the caregivers' attitudes and their family constellations.

The experiences of caregivers are not the same; rather, they vary in their perceptions, their responses to the situations they faced. One of the prominent studies on care practices between family members is Lewis and Meredith (1988)'s qualitative study which investigated the experiences of daughters who cared for their mothers. They divided the daughters into three types - the balancers, the integrators and the immersers - according to their responses to caring for their mothers. The balancers experienced challenges to manage both caring for their mother and their life outside this care, for instance, working in the 
labour market, supporting other family members, including their husbands and their children. On the contrary, the integrators could harmonise their work and their care for their mothers, and almost all the cases of integrators in their study were single daughters who had lived together with their mothers. Lastly, the immersers cared for their mothers exclusively and they were likely to be isolated. They had tried to balance care and work, but it did not work, so they had to give up everything except caring for their mothers. This study shows that caregivers experience different challenges in their caring for their mothers and the supports and helps for elderly care have to distinguish between them according to their response to the challenges the family caregivers face.

Care is not only about interactions between two individuals; instead, there are interactions among family members around caregivers and care receivers. By whom, where and how the elderly are cared for depends on decision-making at the family level, which is likely to be affected by the family's values, its history and social norms. Although elderly care practices are based on moral values and social norms, care practices are a result of continuous negotiations among family members. Finch and Mason (1993) argue that family care is not given by nature but is constructed as a result of negotiations about what is 'the proper thing to do'. Caring for an old parent is constructed in certain circumstances through negotiated commitments and moral reasoning about the proper thing to do.

Although the manner of care is a result of negotiations among family members around caregivers and care receivers, care tasks in daily life belong to one primary caregiver, in that tasks for elderly care are likely to be continuous, so that it is hard to divide the tasks among family members. Nevertheless, care responsibility could be divided in other ways, for example, providing financial and emotional support for the caregivers as a recognition of their caregiving among other family members.

In contrast to European countries, in Korea, the shift in the process whereby family care functions from the private to the public sphere has been incomplete. Many of the care needs have not yet been met by the LTCI programme. What is the situation in Korea? What challenges remain? How do family members make decisions on forms of elderly care after the implementation of the LTCI? 


\subsection{Conclusion}

This chapter has reviewed the research on how elderly care is provided among different actors in society at both the macro- and the micro-level. To understand family caregiving, it is necessary to understand the concept of responsibility for elderly care. Care is a combination of labour and relational practice and the term 'responsibility' is an important concept to capture the characteristics of care. Especially, the feeling of responsibility is one of the most important starting points and motivations in family care. The care responsibility functions and is shared at various levels: between two individuals, among family members, and between the state and the market.

Although most of the care work takes place between two individuals, care has various social characteristics. The concept of 'social care' enables us to understand the social context of care. The responsibility for elderly care cannot be taken by an individual at home alone in an ageing society where the family structure and traditional care attitudes are changing. Therefore, society shares the care responsibility with the family, so that care could be one of the essential criteria for analysing welfare states. For analysing welfare states, the care arrangements are applied as an analytic framework. The framework of care arrangements shows how the responsibilities and resources regarding care are distributed among the state, the market and the family. Besides, even if the same institution is implemented, the results of the institution vary according to the socio-cultural context in society.

Meanwhile, although the state implements public care provisions, the family still plays a significant role in care in that care is a form of complicated and relational labour. Therefore, the state tries to involve the family as an actor in the formal setting of elderly care provision. To encourage the family to offer elderly care, the state puts in place measures such as care leave, credits for pension entitlements and care allowances. However, the position of family carers becomes a blurred one given that the forms of recognition for family care vary among societies. Family caregivers exist on the boundary between formal and informal spheres. In some cases, the state puts the family in the blurred area within the formal institutions. Moreover, the family itself is changing in its structure and culture. Therefore, it becomes hard to figure out how the family should act in the formal care provision system, and it is necessary to investigate how family caregivers experience formal care provision. 
At a micro-level, studies on elderly care practice among family members focus on the experience of family caregivers' challenges such as care burdens and stresses in caring. Caregivers experience different challenges which depend on their position in the family and their relationship with the elderly and with other family members. This is because of the relational characteristics of care. Care practice affects not only the elderly but also the caregivers as an interaction between two people. The family caregivers experience both positive and negative reactions. Besides, although elderly care is offered mainly by one primary caregiver, the care practice is a result of continuous negotiations on who cares and how the family cares for the family member.

Although the FCHs are a significant element that shows the family centred nature of the care culture in Korea, there has been little research about the FCHs. While there has been researched on the informal family caregivers and care work after the implementation of the LTCI in 2008 (Hwang Bo-Ram et al. 2015; Hwang Ji-Seon et al. 2015; Kang Tae-Wha et al. 2014; Seo Insoon 2003), there has been less focus on the experience of the family care helpers, who are licenced family caregivers for the elderly. Most of the research on the FCHs is about patterns of LTC services compared to non-familial care workers (Lee YunKyung 2010, Hong Sung-Wook 2011), and these empirical studies mainly aim to investigate whether the FCHs ask for unnecessarily high fees, which hinders the sustainability of the LTC finances. Few studies focus on the experience of the FCHs (Choi In-Hee and Kim Jung-Hyun 2013; Jeon Byeongjoo and Kim Hyunsoo 2016; Lee Min-Sook 2012; Yang Nan-Joo 2013). Lee Min-Sook (2012), in her investigation of the experience of the FCHs, argues that most of the FCHs who were interviewed were committed to caring for their family members. In other words, not all the FCHs tried to misemploy the payments for elderly care and service regulations.

To formulate my theoretical arguments based on the literature review above, I here recall my initial assumptions in Chapter I (Introduction):

1) The social status of the FCHs would be situated somewhere between workers and family members.

2) Through the introduction of the LTCI programme, the FCHs could be concerned about their social rights as individual workers, for example, their entitlement to the national pension system (NPS), to unemployment insurance and to occupational insurance. 
3) At the same time, the FCHs might feel that it is unfair that they have to take on all tasks for the elderly since they are 'licenced and paid' caregivers from the LTCI programme.

Considering these assumptions, I set out the empirical questions and travelled to do the fieldwork with these questions in mind: How did informal family caregivers decide to become FCHs? What is the lived experience of the FCHs in both public and domestic areas? How do they experience becoming and being a FCH in terms of their social status and social rights? How do they cope with the new challenges of being situated between their status as workers and as family members?

Specifically, there is a need to investigate the process of becoming and being a FCH, which will enable us to get insights into how the positive and negative factors in caring for the elderly are interwoven throughout everyday experiences and how the traditional care culture in Korea affects the care responsibility among actors at two different levels (macroand micro-). The empirical findings of this study will be presented in Chapter V (Results). Before showing the results, however, I will discuss the methodological framework and the methods applied to the empirical qualitative investigation in the next chapter IV (Methods). 


\section{CHAPTER IV THE METHODS}

\subsection{Introduction}

This chapter focuses on a methodological framework and the methods applied when seeking answers to my research questions posed in chapter I. As a methodological framework, I applied a qualitative approach which is designed to access family care helpers' (FCHs') experiences in everyday life as well as generate new theories (Reinharz and Davidman 1992). For data collection, this study uses the problem-centred interview (PCI, Witzel and Reiter 2012) method, which has been designed particularly for interviews dealing with socially relevant problems. To analyse the interviews I conducted, a biographical analysis was applied. I interpreted the experiences of interviewees according to a biography-oriented analysis of Rosenthal (1995; 2011; 2018)'s suggestion and established case reconstructions of three exemplary cases, which represented three types of the FCHs in response to care responsibility for their old family member. The biographical research provides a possibility to exceed traditional methodological dichotomies of individual/society, subjectivity/objectivity, and structure/agent through the individual's lived experience in the life course (Yi Hee-Young 2005: 121ff.).

In the following section (4.2), I discuss the rationale of the qualitative approach and explain the context of the study, i.e. providing an argument for the selection of FCHs in the LTCI in Korea. Section 4.3 details the research design, including the sampling strategy, the way in which the in-depth interviews were conducted, and the ethical considerations of the research process. Section 4.4 represents biographical case reconstruction as a strategy of data analysis, including the selection of the cases among the interviews conducted as well as the description of steps of the analysis. Finally, I conclude and summarize the contents of this chapter in section 4.5 .

\subsection{Rationale for the Qualitative Approach}

The objective of this study is to investigate the FCHs' experience of caring for their older family member(s). Given that the caregiving could be understood in the sense of family practice, the subject of this study is related to individuals' subjective meaning and values 
in the course of interaction among family members. In addition, the FCHs care for the elderly in a home environment, so it would be hard to separate their caregiving for the elderly from their daily life as a whole. Furthermore, previous research has shed little light on the experiences of FCHs in Korea, thus, an exploratory study on these experiences is called for. The qualitative approach enables to grasp a complicated social phenomenon from the perspective of the individual by focusing on how the individual experiences the social phenomenon and responds to challenges they face in society in their everyday lives (Yi Hee-Young 2005: 134ff.).

Qualitative research focusses on the description, context and process of the individual's experiences in everyday life. Therefore, the qualitative methods are used to grasp complex details, such as subtle feelings and thinking processes, which are difficult to understand through conventional quantitative methods (Strauss and Corbin 1998). These methods examine people's understanding and beliefs rather than objective facts, and they focus on context, holism, flexibility, descriptions, and process (Bryman 1992). I worked particularly with the qualitative methods from biographical research which provide a possibility to exceed dichotomies of individual and society, subjectivity and objectivity, as well as structure and agency (Yi Hee-Young 2005: 121ff.).

The biographical research starts from individual experience. One basic assumption of the biographical research is that individual's subjective intended meaning is neither private nor an internal psychological process (Rosenthal 2011: 19). A biography of an individual is a socially structured formation in that the social structure is revealed through the biography of the individual (Fischer-Rosenthal 1996: 149). The individual actively interprets a socially defined 'frame' by 'framing' in the course of social interactions where an intention and meaning are newly constructed (Willems 1996: 444 in Rosenthal 2011: 43). Meanwhile, an individual's experience comes into existence in a certain place and time, and interpretations of former experiences. By giving the example of a possibility of one's narration on the traumatic event, Rosenthal (2018) argues that how one remembers the traumatic experience and one's ability to talk about them (Rosenthal 2018: 42). For this reason, she suggests that a researcher needs to distinguish between interviewee participant's life story (narrated life) and life history (lived life).

Another strength of the biographical research is an openness of the method. The openness is applied at various stages of the process: in research design, data collection, and analysis. 
First of all, a researcher keeps in mind that the research design might need to be modified to cope with emerging unexpected findings during data collection. The openness is also applied in the process of data collection itself. In qualitative approaches, the data collection and analysis follow a circular pattern. This means that preliminary analyses are already carried out during the data collection, and provisional findings affect the former research design and the data collection in the process. On the other hand, when the researcher finds they are missing information in the process of the data analysis, they enter the field again for a more detailed and/or more specific collection of data. In more specific situations, for instance, in the course of an interview, one could adjust the contents and orders of the interview questions according to interviewees' narrations or interactions between the interviewer and the interviewee. The principle of openness is also applied in the process of the interpretive analysis. With the principle of openness, one reflects on whether one's understanding of the research subject and research field changes compared to the initial assumption and the research questions. This approach enables the researcher to meet new themes or to develop new research questions. The interview procedure, which will be explained in detail in section 4.3, is also based on the principle of openness in the qualitative interview.

Based on the methodological stances described above, I consider the relationship between researcher and research. I define a researcher as a well-informed traveller, who wants to visit what remains of society as it existed hundreds of years ago (Witzel and Reiter 2012: 14). The site has changed, and the traveller cannot fully understand the historical ruins since he or she belongs to the contemporary world. Therefore, it is helpful for interviewers to acquire prior knowledge of the research themes and to receive guidance from informed insiders. This means the researcher must reconstruct interactively constructed knowledge. As a researcher, I interact with the social world, and because findings are mediated through my perspectives and values, the findings cannot be value-free (Lewis 2003; Witzel and Reiter 2012). I applied the PCI and the biographical case reconstruction as methods for this empirical study since the PCI method combines an open question for a self-presented narration of the interviewee with topic-guided questions, too. In the next section, I will explain the process of data collection, including a research design, strategies to recruit interviewees and the interview procedure, and will discuss ethical considerations. 


\subsection{Research Design and Data Collection}

Based on the methodological framework described in the previous section, I designed qualitative in-depth interviews for data collection. I conducted 18 problem-centred interviews (PCIs). The PCI method (Witzel 1985; 2000; Witzel and Reiter 2012) is an interview strategy for generating a theory. This method was developed in the 1980s by the German psychologist Andreas Witzel while conducting a research project about job perceptions and the decisions of secondary school students (Witzel 1985). He discussed the main concepts of PCI, such as problem-centring (Problemzentrierung), process orientation (Prozessorientierung), and object orientation (Gegenstandorientierung) (Witzel 1985; Witzel 2000; Witzel and Reiter 2012). The PCI method uses a specific communication strategy, including the formerly noted objective conditions, methodical flexibility and promoting conversation flow (Witzel and Reiter 2012). The PCI is an elaborated method for combining deductive and inductive elements in a flexible interplay and it has been designed particularly for the purpose of in-depth interviews on 'socially relevant problems'.

After I set out the methodological framework (section 4.2), I started to plan my research design and data collection. I decided to apply the PCI for the data collection since the PCI method is designed to combine in one instrument two objects: methodical openness to capture the meanings and subjective framework of an individual as well as focus on topics regarding becoming and being a FCH. In order to achieve openness at the start of the interview, I began with a non-directive input that asks the respondents to provide narration on their life regarding becoming a FCH without any further specifications of which life domains to focus on. I prepared a topic guide to manage both stages of the interview.

\subsubsection{Preparing PCIs}

Before conducting an interview, I prepared research work packages, including a topic guide, an interview schedule sheet, an information sheet for the interview, as well as a contact letter and a consent form for an interview, which participants were given on the site of the interview. A topic guide is a diagram, which acts as a strategy for communication. The guide functions as a road map, which helps the interviewer keep to a problem-centred thematic framework (Witzel and Reiter 2012: 32), and to spot clues when they are confronted with an intricate situation during the PCI process. According to the topic guide, an interview starts with an opening question and follows the main narrative to support the 
narrative string developed by the interviewees. During the open narrative stage of the interview process, I listened carefully to the interviewee's self-presented accounts, paying attention not only to the contents but also to the way how they generate and develop their life story. At the moment of the research design, I could of course not predict where and how the interviewees began with their self-presentation. However, special regard was paid to a self-presentation of the interviewee. I assumed that the interviewees would choose the contents and theme of their story according to their point of view at the moment when they generate their life story, i.e. the interview situation. This means that I had to be aware of differences between an experienced life history (erlebte Lebensgeschichte) and a narrated life story (erzählte Lebensgeschichte) in the sense of Rosenthal (2011;2018)’s suggestion.

In addition to planning an open-narrative question, I formulated interview questions which were related to my research question based on my preliminary literature review. I set out seven topics: 1) the present circumstances of the FCHs; 2) the education and working history of the interviewees; 3) their caring history; 4) their process of becoming a FCH; 5) FCH as a worker under the LTCI; 6) the FCH as a family member; and 7) the meaning of the experiences. I assumed that each topic was relevant to my research topic, but I did not expect that each topic would be assigned to answer a specific question. Rather, I expected which topics might help me to manage the interaction with the interviewee by suggesting provisional thematic fields I prepared before the interview. Figure 4.1 gives an overview of the topic guide (See Figure 4.1 in the next page).

I applied theoretical sampling, which is a specific form of purposive sampling. Unlike quantitative research, which is generally carried out on a representative sample, an interview unit is selected according to the potential contribution to theory development, rather than according to a statistical probability (Lewis 2003: 107). To manage the present status of the data collection, I used an interview schedule sheet, which enabled me to keep a good overview over basic information on the interviewees and the elderly they care for, as well as the way in which contact was established. I also opted for dissimilarity sampling as a sampling strategy, which means that preferably respondents with the background very diverse or different from each other were chosen (Rubin and Rubin 1995: 74). This enabled me to achieve knowledge more broadly and to wield a generalizing argument effectively. Once I recognized a distinguishing pattern, I tried to contact a new interviewee who met the requirements for the principle of the dissimilar sampling (Rubin and Rubin 1995). To 
set out the diversity of the case, the interviewee was recruited according to several criteria concerning: the respondent's status (age, gender, relation to the recipient, working history, and care experience) as well as the elderly's status (age, gender, LTCI grade, and disease type). This sampling was continued until the point of theoretical saturation is reached, i.e. until there emerged no more new or relevant categories or data (Strauss and Corbin 1998). 


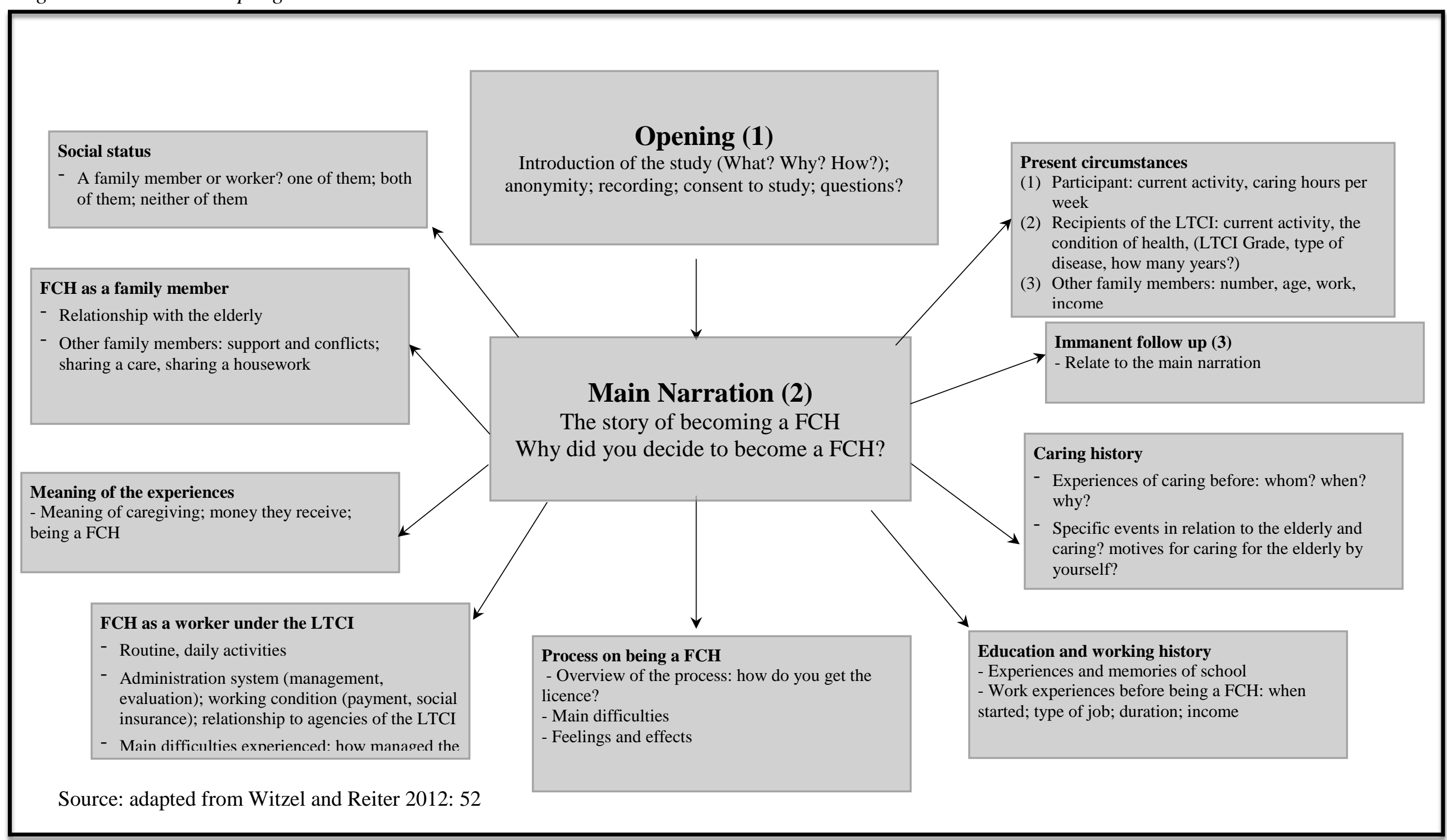


After I determined the interview design and sampling strategy, I planned my fieldwork. Since the research subject of this study is family care helpers (FCHs) who care for their old and frail family members in the long-term care insurance programme in Korea, I had to travel to Korea to meet the interview participants in person. I planned to stay in Korea for my field research for several months in 2015. Before the main phase of fieldwork in Korea, I prepared a first exploratory investigation on the internet and a pre-study, staying in Korea for three weeks in January 2015.

At first, I tried to find potential interviewees over the internet. In order to achieve this, I wrote a request for participation in the study on an internet forum for those with an interest in the LTCI in Korea, targeting care workers and recipients of LTCI, and in-home care agency runners. The internet forum had about 17,000 members and offers information on the LTCI services. However, it soon became obvious that this field access would not bring much success since most of the forum's users checked the forum irregularly and hesitated to participate in an in-depth interview with someone who was not accountable in cyberspace.

Therefore, I decided trying to find more personal field access. I attempted to contact informants who would be able to introduce me to FCHs. In January 2015, during the prestudy phase, I visited to establish initial contact with the informants before meeting actual interviewees in Korea. The informants I wanted to contact were those who could understand the purpose of this study and had networks with potential interviewees; like care workers, nurses, and directors of care helpers' associations. I explained to them the purpose of the interviews and asked if they would be able to introduce me to potential interviewees when I would be back in May 2015 for my main phase of fieldwork. Besides these informants, I also asked interviewees whether they could possibly introduce me to FCHs. This line of inquiry brought little success though since most FCHs seemed to spend the majority of their time at home caring for the elderly and had little contact with other FCHs. Their primary contacts seemed to be the respective social workers who manage the LTC services and whom they usually report to.

Therefore, I recruited the interviewees for this study mainly by the means of informants and personal connections like friends' relatives and relatives' friends. In doing so, I recruited eighteen interviewees who had obtained a licence for a care helper and care for 
elder family members who were recipients of the LTCI; ten of these participants were over 65 and had already retired, and the rest were of working age.

During the field trip, I wrote an interview schedule sheet, which enables me to manage the appointments with informants and possible interview participants. Specifically, I could check at a glance how I found possible interviewees through the informants since I recorded the connection between the informants and the interviewees when I met them. I also recorded cases where the potential interviewee rejected to participate in an interview. Apart from the interview schedule sheet, I wrote an information sheet, which provides basic information of the interviewees, including demographic and social status of both the interview participant and the elderly they cared for. It helped me to decide which interviewees, in particular, I wanted to focus on according to theoretical sampling and maximum contrast (Glaser and Strauss 1967; Schütze 1983).

\subsubsection{Conducting the PCI: the procedure of an interview}

When conducting an interview with a participant, I followed the stages of the PCI method, which are: an introductory explanation and briefing, warming up, the opening question, follow-up questions (to explicate the implicit knowledge), a discussion of social/personal characteristics, and a short questionnaire (Witzel and Reiter 2012: 64). A combination of open and semi-structured interview questions enables to achieve both methodological openness and a specific research focus.

In the introductory stage, I briefly informed the interviewee about what to expect from the interview. I explained the context and purposes of my research from my point of view and informed them about the process of the interview, such as the process of recording and documentation, as well as the rights of the interviewee, and the fact they would receive a small amount of money as a reward. Additionally, I offered this explanation to the interviews in a contact letter (see Appendix 1) to ensure easy understanding. Then I gave the interviewees about two minutes with a consent form (Appendix 2) to decide whether to participate in the interview. When the interviewee decided to take part, I started to question the interviewee according to the PCI method. After asking a few questions on the demographic status of the interviewee and the elderly they care for, I posted the opening question, designed to help to generate the interviewee's main narration. This question was phrased as follows: 
"Please tell me your story about the elderly's health condition and how you decided to become a family care helper"

After a few interviews, I realised that answers to the opening question varied at the moment they took as a starting point of their story. For example, while daughters-in-law were likely to begin their narrations with the very moment when they became aware of changes in the elderly's condition, daughters and wives were likely to start their narrations with some episodes before the elderly had started to get frail. During the interviewee's main narration, I tried not to interrupt the interviewee but instead to listen carefully and take notes about keywords for follow-up questions. Furthermore, I showed empathy and interest through short verbal signals such as 'ah' 'hmm' 'yes' as well as non-verbal communications like facial expressions, gestures and postures during the interview.

After the narration of the interviewees, I posed questions in order to achieve the second objective, which was focusing on central topics based on the initial assumptions of this study (see Chapter I) and provisional literature review. I applied the topic guide which consists of seven blocks of questions (see section 4.3.1). The order and sequence of the questions were flexible and adjusted to the respective narrative flow. After the interview, I wrote a postscript (or memo) as a supplement tool. I described my observations and impressions of the interview, like non-verbal information. The postscripts thus serve to capture hints and details that do not have the place in a literal transcript of an interview and/or cannot be conserved by means of a voice recorder.

Between May and August 2015, I conducted eighteen interviews with FCHs in Korea. Most of the interviewees lived in Seoul and around the metropolitan area, only one daughter-inlaw lived in a rural area. Two of the eighteen interview participants were men. At first, I planned to interview female FCHs only, but I needed to identify whether there were differences in the care responsibility and cultural norms among son, daughter, and daughter-in-law as well as between wife and husband. After my main fieldwork in 2015, I decided to have another field trip for follow-up interviews with three interviewees whom I had made out to be representative for case types in the study. The follow-up interviews were conducted in November 2017. During the two research field trip to Korea, I 
interviewed 18 family care helpers. The following table (Table 4.1) lists the candidates with the information available by the time of the interview ${ }^{11}$.

Table 4.1. A profile of interviewees who participated in the study

\begin{tabular}{|c|c|c|c|c|c|c|c|}
\hline & Age(birth) & $\begin{array}{l}\mathrm{Se} \\
\mathrm{x}\end{array}$ & $\begin{array}{c}\text { Years of } \\
\text { caregivi } \\
\text { ng }\end{array}$ & $\begin{array}{c}\text { Years of } \\
\text { caregiving } \\
\text { as a FCH }\end{array}$ & Disease $^{1)}$ & $\begin{array}{l}\text { Cares/ed } \\
\text { for }^{3)}\end{array}$ & Current living arrangements \\
\hline 1 & 68(1947) & $\mathrm{F}$ & 20 & 5 & Dy, St & Husband & Co-resident \\
\hline 2 & $55(1960)$ & $\mathrm{F}$ & 6 & 6 & $\begin{array}{l}\text { Dy, St } \\
\text { Di, Dp }\end{array}$ & $\begin{array}{l}\text { F-in-law } \\
\text { M-in-law }\end{array}$ & Co-resident \\
\hline 3 & $44(1971)$ & F & 15 & 5 & $\mathrm{Hi}$ & M-in-law & Co-resident \\
\hline 4 & 66(1949) & $\mathrm{F}$ & 20 & 6 & $\mathrm{Mu}$ & Husband & Co-resident \\
\hline 5 & 56(1959) & $\mathrm{F}$ & 3 & 3 & St & Brother-in-law & Separate \\
\hline 6 & 54(1961) & $\mathrm{F}$ & 3 & 3 & Di, Hi & M-in-law & Separate \\
\hline 7 & 63(1952) & $\mathrm{F}$ & 4 & 4 & $\mathrm{Al}, \mathrm{Pa}$ & Husband & Co-resident \\
\hline 8 & 74(1941) & $\mathrm{F}$ & 7 & 6 & St & Husband & Co-resident \\
\hline 9 & $55(1960)$ & $\mathrm{F}$ & 2 & 1 & Hi, Di & Mother & Separate -> co-resident \\
\hline 10 & 74(1941) & $\mathrm{F}$ & 23 & 4 & St & Husband & Co-resident \\
\hline 11 & 73(1942) & M & 10 & 3 & Di, Hi & Mother & Co-resident \\
\hline 12 & 59(1956) & $\mathrm{F}$ & 20 & 7 & Di, Hi & Mother & Separate \\
\hline 13 & 70(1945) & M & 21 & 5 & $\mathrm{Br}, \mathrm{He}$ & Wife & Co-resident \\
\hline 14 & $55(1960)$ & $\mathrm{F}$ & 2 & 2 & $\begin{array}{l}\text { Dy, St, My } \\
\mathrm{Hi}, \mathrm{My}, \mathrm{Di}\end{array}$ & $\begin{array}{l}\text { F-in-law } \\
\text { M-in-law }\end{array}$ & Separate -> co-resident \\
\hline 15 & 54(1961) & $\mathrm{F}$ & 5 & 1 & $\mathrm{Al}, \mathrm{Pa}$ & M-in-law & Separate -> co-resident \\
\hline 16 & 72(1943) & $\mathrm{F}$ & 18 & 2 & St & Husband & Co-resident \\
\hline 17 & 60(1955) & $\mathrm{F}$ & 5 & 0.5 & Ar, Di & Mother & Co-resident \\
\hline 18 & 54(1961) & $\mathrm{F}$ & 3 & 2 & $\mathrm{Di}$ & Mother-in-law & Co-resident \\
\hline
\end{tabular}

Source: Self-elaboration based on field notes

Note: 1) Al: Alzheimer, Ar: arthritis Br: brain lesions, Di: dementia, Dp: depression, Dy: dibasic, He: hearing-impaired, Hi: hip fracture, Mu: rare muscle disease, My: myocardial infarction Pa: Parkinson's disease, St: stroke 2) F-in-law: father-in-law, M-in-law: mother-in-law.

11 The interviews on the list were conducted in Korea, between May and August 2015 and November 2017. Although I had done another field trip for a pre-study at the beginning of 2015, I had not been allowed to use the interview for my research. At that time, I conducted an in-depth interview with one family care helper who was a daughter caring for her old mother. However, after a few days, the interviewee withdrew consent to participate in the study and I, therefore, ruled her out of the list of the candidates for the analysis. 
All interviews were carried out, recorded, transcribed, and coded by myself. Each document was labelled with document numbers and line numbers, which helps to find the original interview text and to comprehend the context of the accounts of interviewees. The working language of the study was Korean, since all respondents were native Korean speakers, as am I. The interviews were recorded and transcribed in the original language and then translated into English when necessary. All voice records of the interviews were transcribed into documents, which mainly contained conversations, pauses, interruptions as well as non-verbal expressions such as laughing and crying. If necessary, intonations, stresses, and stops were displayed in certain accounts of interviewees to clarify their expression more vividly. Table 4.2 provides an explanation of the punctuation and transcript signs used for transcribing the interviews (cf. Gibbs 2008: 10ff.):

Table 4.2. Transcript Notation

\begin{tabular}{ll}
\hline \multicolumn{1}{c}{ Transcript form } & \multicolumn{1}{c}{ Signs } \\
\hline (Aha) or (I see) & $\begin{array}{l}\text { Brief pauses; the number of commas represents the length of the pause } \\
\text { Overlap in the accounts of interview partners; chiming in with other } \\
\text { accounts } \\
\text { Claughs] or [crying] }\end{array}$ \\
$\begin{array}{l}\text { Comments on paralinguistic features } \\
\text { ([laughs]) }\end{array}$ & $\begin{array}{l}\text { Overlap of the paralinguistic features of interview partners } \\
\text { ? }\end{array}$ \\
CAPITAL LETTER & $\begin{array}{l}\text { Speaking loudly } \\
\text { Underline }\end{array}$ \\
\hline
\end{tabular}

Source: Gibbs 2008: 10ff.

\subsubsection{Ethical considerations for the interviews}

Unlike natural sciences, social sciences focus on social phenomena and the interactions between people and society. For this reason, empirical studies in social sciences deal with human behaviour and direct or indirect feelings. As an interaction between researcher and interview participants inevitably takes place in the course of data collection and analysis, the ethical standards of qualitative research are important. In the following, I will consider and address several ethical issues in the process of interviewing and analysis. 
I assume that the relationship between the researcher and the researched can be determined by the information asymmetries in an interview setting. To allow interviewees to participate actively, certain aspects and possible consequences of the research must be explained, so the researcher can make sure that the participants can give informed consent to the proceedings. Before each interview, I briefly explained the purpose, range, context, specific process, and applications of the research. Furthermore, the participants were informed that they may pause or quit the interview whenever they did not wish to continue. Specifically, I gave a contact letter (Appendix 1) and a consent form (Appendix 2) which required the signatures of both the researcher and the interviewee.

Another ethical issue that presented itself in the course of the interviews was the location where I met the interviewees. Since most of the care tasks of FCHs are performed in a domestic environment, I preferred to conduct interviews at home of the participants so that I could observe the conditions and atmosphere of the place. The house can be assumed to be meaningful to people working as FCHs as an overlapping area of public and private, labour and rest. At the same time, the home is, of course, a very private and intimate place to the interviewees, and they might not want to invite me there. For this reason, I conducted an interview at home or at a private place only with the consent of the respondents. Usually, I first contacted the potential interviewees on the phone and made an appointment with them. At that moment, I carefully asked about the place and time that would be convenient for them, without showing my own preferences. Some of the interviewees invited me to their homes, while others preferred to meet me at more neutral places like a café. Only one interview was carried out in an office room of the care centre the person was registered at. The room was separated from the others, but the social workers and director of the care centre worked in the rooms next door. In settings like this, it has to be considered how the proximity of superiors can affect the respondents and their feeling of security and confidentiality. After this interview, I, therefore, decided to avoid office rooms associated with supervisory institutions in the interest of the interviewees.

To prevent harm to interview participants, all the names used here are pseudonyms. If needed, the names of cities, proper pronouns, and trademarks that could specify the informants or the interview participants were removed or changed. Power relations between family members were also considered since some topics can cause conflict between family members and interviewees. For example, eleven of the eighteen interviews in my study 
were carried out in the interviewee's house since most of them had to stay at the form for the elderly they cared for. Sometimes, I met the elderly and tried not to explain all about the purpose of my visiting unless the interviewee's permission. When an interviewee wanted me to explain about the interview to other family members, then I gave enough information to make a decision on participation in the interview. When I conducted an interview at home, I suggested a separate space for the interview to the interviewees.

Before my analysis, I documented the collected data. At first, all materials were recorded to data in several ways. All interview materials, including mp3-formatted voice files, transcripts, postscripts and memos were documented and stored. Since these materials are the main sources of the analysis of this study, they had to be well organized to be easily accessible. I wrote postscripts, which consist of information about an interview, including its date and duration, as well as the main structures, contents, scenes, and images used during the interview. The postscripts can also be used for the analysis since they contain unrecorded information in the voice files to remind the researcher of the situation (Witzel and Reiter 2012: 95ff.). Basic information on the interviewees, including family trees and household income and expenses, were generated based on the interview transcripts, short questionnaires and postscripts.

\subsection{Data Analysis: from topic-oriented to case reconstructions}

After the data collection and documentation, I had to decide which methods would be best suited for my analysis. This study aims to build theory through an interpretation of the experience of the FCHs in the LTCI by constituting, examining, and refining the collected data (Witzel and Reiter 2012: 99ff). At first, I intended to apply the topic-oriented evaluation drawing from Witzel and Reiter (2012), but in the end, I decided to utilize the biographical case reconstruction as suggested by Rosenthal (1995; 2011) instead. In the following, I will describe the process which lead me to this decision and why I deem the biographical case reconstruction the best fit for my specific aims.

Witzel and Reiter (2012) suggest two different strategies for analysis of PCI interviews: a topic-orientated analysis and a biography-oriented analysis. At first, I decided to apply the topic-oriented analysis, since I had conducted the interviews using a topic guide that included (as described earlier) topics like: the process of becoming a FCH, the negotiations on the elderly care among family members, and the usage of the payments. I expected that 
the preliminary categories from the topic guide would help me to focus on specific areas of my research interests and to find salient patterns and themes through the comparisons of the cases.

For this topic-oriented analysis, I narrowed down the interpretation of certain issues and divided the process into vertical and horizontal evaluations. In the phase of the vertical evaluation, I analysed the contents of a certain interview, utilizing the strategies of continuous questioning and coding. First, I read the transcripts asking the material questions like 'what is going on here?', 'What does the interviewee notice about the phenomenon?', and 'what are the structural conditions which a certain situation involves?' (Corbin and Strauss 2008: 100). I also applied the method of coding that involves breaking down, comparing and categorizing of the interview transcripts. I read each transcript several times to capture important topics by asking questions by myself. In particular, I went through the transcript line by line to infer the intention and meaning of the interviewees' statements from their perspective, looking for salient accounts, which could help explain the situation and the phenomenon. I labelled them with a specific code or assigned a category according to the interview topic guide. The naming of these codes was derived both from existing theories and from in-vivo statements, meaning a word, a sentence, or even a number of sentences found in the interviewee's accounts. Furthermore, one account can be labelled with more than one code. Through the vertical evaluations of the cases, I compiled 18 case summary files.

After the initial coding process and the vertical evaluations, I tried to proceed onto case comparisons to find a "hypothetical relationship between categories and properties" (Corbin and Strauss 2008) and connections between categories and sub-categories (Witzel and Reiter 2012). I then tried to organise and to elaborate the categories by analysing the causal conditions and context of the codes. I applied a 'category system' (Kühn and Witzel 2000) based on the topic guide used in the PCIs. The preliminary categories were also generated based on the previous theories and empirical studies regarding my research questions. Interviewees' statements were assigned to a certain preliminary category as 'a container of the meaning unit' (Kühn and Witzel 2000). After setting up the categories, I constantly tested their quality by means of iterative reflection. If a category was too narrow or too wide, I modified its range. 
However, it became clear that the topic-oriented analysis did not suffice to answer my research questions for several reasons. Firstly, the experiences of the FCHs in caregiving are interwoven with the caregiver's life course. That is why when I broke down a whole story of an interviewee into categories, I could not capture the contexts and backgrounds which are embedded in the excerpts. Moreover, I needed to understand not only the contents of the interviewee's accounts but also the way the interviewees generate their narrations which could reveal the latent meaning and structure of the text and provide more plausible explanations of the experience of the FCHs. Therefore, I changed my strategy for the interpretation of the material and shifted the focus towards the whole biography of the interviewee. Despite this change of strategy, some results of the vertical analysis still could be applied in a useful way during the stages of microanalysis and case reconstruction of the biographical analysis.

A biography-oriented analysis focuses on how a person generates a story based on interpretations of his/her experiences and reconstructs interpretive patterns that are typical for the case. As the process of the case reconstructions begins with analysing a certain case, I had to decide from which interview to start my analysis. To decide the first analysis, I read postscripts and memos which I generated and used from the interviews and the earlier analysis. I chose the interview with Anmi, a daughter-in-law caring for her mother-in-law, since she is a traditional caregiver in the sense of son's obligation in Korean care culture and expressed intensive feelings about relationships with the elderly and her extended family members including her husband's and her own siblings.

Anmi was my last of 18 interviewees of my fieldwork in South Korea in 2015. I had already interviewed 10 adult children who cared for their parents with qualifications as care helpers with the LTCI. During that field trip, I preliminarily categorised the adult children into two groups according to whether an interviewee was a lineal descendant or not, since I assumed that a linear family relationship (like daughter-mother, or son-mother) might affect their caregiving differently compared to non-linear relationships (like daughters-in-law). As I conducted the interviews, however, I noticed that there were other and more important factors, for instance, the history of relations between the caregiver and the care receiver, attitudes and motivations towards caring for parents, and a caregiver's economic status.

In addition to Anmi's case, I chose another case for reconstruction, according to the principle of the maximum contrast (Glaser and Strauss 1967; Schütze 1983). Sunjoo’s case 
represented the opposite site of Anmi's case in that, as a daughter of the elderly, Sunjoo was not a traditional caregiver in the sense of Korean care culture, but rather represents a new trend in the country, the caregiving from daughters. The third interview that I decided to reconstruct was Jina's. While the former two cases of Anmi and Sunjoo represent adult children (in-law) of the elderly, Jina is the spouse of the elderly and older than the former two cases.

After having decided on these three cases, I began the biographical case reconstructions following the analytical steps listed below (Rosenthal 2018: 168-186).

\section{Analytical steps for case reconstructions (Rosenthal 2018: 166)}

1. Analysis of the biographical data (event data, including historical data): preparation for step 3

2. Thematic field analysis (structure of self-presentation; reconstruction of the narrated life story)

3. Reconstruction of the experienced life history

4. Microanalysis of individual text segments (can be carried out at any point during the analysis)

5. Contrastive comparison of life history and life story

6. Development of types and contrastive comparison of several cases

The first step of the analysis is to arrange events mentioned in the account of the selected interviewee, who is also called a biographer in the sense of Rosenthal's suggestion (2018), with considerations of the temporal-biographical order of events. I made an executive summary in chronological order which starts with the date of birth, family backgrounds and social constellations with considerations on the interviewee's age and cognitive development. Furthermore, the summary includes information on the elderly the interviewee cares for, including types of diseases and care Grades of the elderly, living arrangements and the formal relationship with the elderly. The executive summary is a description of the objective incidents in the biographer's life without the interviewee's own statement, which means the summary is not subject to the interviewee's interpretation. This step of analysis provides an overview of the past and current life events of the interviewee. It is meant to and helps the researcher to regard the case with a certain degree of distance from the manner in which the interviewee presented his/her life.

I then read the summary sentence by sentence and set out hypotheses and follow-up hypotheses concerning "what could happen in the life of the biographer to make changes possible” (Rosenthal 2018: 169). These hypotheses and follow-up hypotheses were 
prepared for the case reconstructions of the experienced life history (step 3). During analysis, I tried to view the text without any prejudices or background knowledge. Although I conducted all interviews and wrote the summaries by myself, I tried to put aside my previous knowledge about the interviewees in accordance with the principle of the sequential and abductive method. This was helped by the fact, that I could indeed not remember all parts of the interviews in detail. Furthermore, the methodological requirement to list all possible hypotheses and follow-up hypotheses about what could happen next in the biographer's life ensures that the researcher does not concentrate on one reading he/she may know (or think) to be true.

After assembling this executive summary of the interviewee's biographical data, I went forward with the text and thematic field analysis (step 2) by applying a sequential analysis of textual segments. To prepare this analysis, the text is sequenced in chronological order and divided into analytical units. These units are, however, deduced from the text itself and there are three criteria for deciding when a sequence begins and ends: change of speaker, change of textual sort (argumentation, description, narration) and change of content (cf. Rosenthal 2018: 177).

While the analysis of the biographical data (step 1) focuses on the objective events the interviewee experienced in the past, the thematic analysis concentrates rather on the interviewee's presentation of these events, which gives us some indication of their present perspective from which they interpret these events. The sequential analysis enables the researcher not only to make clear contents on a certain topic but also to show how the interviewee (sub)consciously employs framing by choosing or avoiding certain themes or topics (cf. Rosenthal 2011: 72). Specifically, I focused on the main narration of the interviewee, meaning the part of the text I got as an answer to the open first question of the interview, before asking further questions. Although I always asked the same question, the interviewees started their narration in different ways, at different phases of their lives. I formulated hypotheses and follow-up hypotheses on why the interviewees began in a certain way and not different.

In the next step, I built a reconstruction of the interviewee's experienced life story (step 3) by comparing the biographical data to the thematic field analysis. This is meant to help us find the interviewee's past perspectives on the events, in other words, the perceptions that are close to their actual experiencing. Through this analytical process of the case 
reconstructions, I could verify the hypotheses and the follow-up hypotheses, which I set up in the first and second steps. Moreover, the case reconstructions helped me to formulate new interpretations on the experienced life story of the interviewees and to discover significant biographical experiences which may not have been worked out in the previous steps. Besides, I had to keep in mind that although I synthesised the meaning form the data I collected, I could back into the context of the raw data when needed of microanalysis. In the process of the analysis, the researcher becomes an instrument by interpreting the meaning of the text, which means the experiences and theoretical frameworks of the researcher automatically become embedded in his/her interpretations (Kim Insuk 2016: 308). The reconstructions of three cases (Anmi, Sunjoo, and Jina) will be presented in the following chapter (V).

In the course of the analyses, I used the microanalysis (step 4) in order to "reveal the latent meaning and structure of the text" (Rosenthal 2018: 185). I selected passages, which were unclear or contained striking paralinguistic features such as long pauses, slips of the tongue, or incomplete sentences. After having identified passages like this in the interview transcript, I broke them down into very small units and examined them intensively unit by unit according to the overall gestalt of the text. With the microanalysis, I could test and expand the hypotheses and plausible interpretations by analysing the text material in more detail and possibly finding latent meanings that were not revealed during other steps of the process. However, when new hypothesis emerged, I put aside interpretations I set out in the previous steps and tried to formulate all possible hypotheses and follow-up hypotheses, in order to better understand the phenomenon. This approach is derived from the principle of the abductive approach - which has already been referred to before, and which begins with hypotheses and verifies them inductively based on the empirical data. The microanalysis can be applied at any stage of the analysis. Therefore, I employed the strategy when I needed an intensive detailed analysis to generate plausible interpretations and reveal latent meanings that were not obvious to me before.

Apart from the microanalysis, I wrote and read memos throughout the data collection and the analysis, which play a significant role in the analysis. Glaser and Holten explain that:

"Memos are theoretical notes about the data and the conceptual connections between categories. The writing of theoretical memos is the core stage in the process of generating theory" (Glaser and Holten 2004: 60) 
Memos are a way of recording and reflecting on the interview and the analysis, I read not only the interview transcripts but also these memos to find patterns and themes. I continuously wrote memos, collecting my ideas about the analysis, concepts and theories. If needed, I went back to the original statements which a memo pointed out to compare the meaning in the overall context of the text. Through these processes, I could determine gaps between things already I knew and things I still had to find out. I then refined, synthesized and compressed the memos.

During the analysis, the meaning of certain words mentioned by interviewees was investigated. These words can be understood in a social and cultural context. For instance, there were many words which describe caring activities. I focused on the word chosen during the interview and on the context(s) this word was applied in. In doing so, I tried to find the patterns and think deeply what the accounts of the interviewee mean.

After the case reconstructions (step 3), I compared the narrative life story and the experienced life history (step 5), to find possible explanations for the experienced of the FCHs. With this comparison, I tried to find rules for the differences between the life story and the life history of the interviewee. Despite the differences, the narrated life story and experienced life history have temporal and thematic relevance, so a certain experience of the interviewee could affect the way they present it and vice versa.

The last step of the analysis is the formation of types (step 6), which represents a theoretical generalisation. In this stage, I worked on an abstract level and came back to the research questions. With the patterns I had found during the previous stages, I established types which help to comprehend the themes. When comparing the cases and focusing on their patterns, the theme emerges and reveals the quintessence of the phenomenon. The concept of the theme refers to a central idea through the phenomenon, and the theme of the phenomenon comes not from existing theories but from the data itself. Since the theme is abstract and embedded in the data, it does not emerge in an early stage. Rather, it emerges when the researcher stands back and looks at the phenomenon as a whole. Throughout the steps of the analysis, I found not only themes I was interested in at the beginning and which I formulated in my research questions, but also new themes and possible research topics to help build the construction of a typology. 


\subsection{Conclusion}

For the purpose of answering the research questions raised in the literature review, a qualitative approach and methods were chosen for the means of analysis and justifications for these decisions were given. Since this study focuses on the perception and experiences of FCHs, I determined a qualitative approach to be best suited to answer my questions. I began with the biography of an individual since the biography is a socially structured formation. For data collection, I applied the PCI method. This method utilises interviews with two phases and makes it possible to achieve two different objectives, getting a selfgenerated narration of the interviewee and applying a preliminary map of the topic in order to obtain data on more specific themes. I paid special attention to the procedure of the interview itself, which was flexible in the contents and order of questions. During the fieldwork in 2015 and 2017, I conducted 18 interviews in accordance with the PCI method.

For analysing the collected data, I followed the method of case reconstruction as suggested by Rosenthal (1995; 2011; 2018). This analysis comprises six steps, including analysis of the biographical data, thematic field analysis, case reconstruction of the experienced life history microanalysis of individual text segments, contrastive comparison of life history and life story, and development of types and a contrastive comparison of several cases. These analytical steps were utilized to provide a better understanding of the experiences of FCHs. Based on the data collection and the analysis I described in this chapter (IV), the next chapter will present empirical findings and case reconstructions of the three selected interviewees in detail. 


\section{CHAPTER V RESULTS}

\subsection{Introduction}

This chapter provides the empirical findings on the basis of which I want to answer the research questions I proposed in chapter I. I investigated how family caregivers experience on their caregiving for the elderly as a FCH. And in this Chapter, I will show three case reconstructions which represent three types I found in the analysis of the interviews I conducted.

With the case reconstructions of the three interviewees, I suggest a topology of the FCHs according to their position in negotiations with their family members on elderly care. Initially, I began analysing cases on basis of family relations as suggested by several previous studies focusing on the influences of family relations on caregiving (Han KyungHae and Lee Seo-Youn 2009; Kim Jiyeon and Kim Hongsoo 2016; Namgung MyoungHee 2010; Paik Jina 2015; Seo Insoon 2003; Shon Yong-Jin 2012). Accordingly, I preliminarily categorised my 18 interviewees into three groups. Firstly, I distinguished the spouses (wives and husband of the elderly) from the adult children and children-in-law (daughters, sons, daughters-in-law of the elderly). Then I differentiated the adult children again into two groups according to whether they were a lineal descendant or not, since I assumed that lineal family relationships (like daughter-mother, or son-mother) might affect their caregiving more positively compared to non-linear relationships (like daughters-in-law). As I conducted the interviews, however, I noticed that there were more important factors than just family relations, for instance, the history of relations between the caregiver and the care receiver, attitudes and motivations towards caring for parents, and a caregiver's economic status. I, therefore, tried to re-categorise the groups considering the new findings raised by the interviews.

The following three sections 5.2, 5.3 and 5.4 present three case reconstructions of three different types that I found to be representative of FCHs and their experiences: traditionalists (5.2), negotiators (5.3) and partners (5.4). Each of the three chapters comprises descriptive and analytic sections, including 1) interview context and making 
contact with the interviewee, 2) a description of the interviewee's self-presentation (on the narrated life story) ${ }^{12}$ in the first phase of the interview, 3) a reconstruction of the interviewee's life history, and 4) structural features summary (on the experienced life history) and a brief summary of the type the interviewee represents. This methodical approach helps to comprehend how the FCHs experience caring for their old family members in daily life13. The section concludes with a discussion of the implications the typology of this study raises by summarizing and contrasting the types. Furthermore, there will be a focus on the process of how the interviewees became FCHs to give us a mean of analysis of how political policies and their consequences play out in the everyday life of people caring for the elderly.

\subsection{Type A: Traditionalists}

This section aims to investigate the experiences of the FCHs by exploring Anmi's experience in caring for her mother-in-law.

Section 5.2.1 describes the context of the interview and how I made contact with Anmi. Section 5.2.2 contains Anmi's self-presentation, while in section 5.2.3 I identify several key themes that are central for understanding Anmi's experiences as a FCH. Here; I reconstruct Anmi's life story according to the patterns I found in the interview with her. Section 5.2.4 summaries the case of Anmi and presents the type represented by Anmi.

\subsubsection{Interview context}

\footnotetext{
${ }^{12}$ Although I followed the method of analysis as suggested by biographical research (Rosenthal 1995; 2011; 2018), the main narrations of the interviews were relatively short compared to conventional narrative interviews. This was because my opening question was more closed (focused on the process of becoming a FCH and the illness of the elderly) than the favoured option for narrative interviews, which is the most open form asking the interviewee to tell their whole family and life story. At that time, I had not yet decided which method I would apply for the analysis of my data. Despite the relatively short narrations in the first phase of my interviews, the interviewees went back to the topic and repeatedly show patterns of the way of speaking in the second phase of the interview. According to the PCI interview strategy (Witzel and Reiter 2012), the second phase of the interview is not semi-structured but rather flexible in content and order of the questions, following the interview flow given that in the PCI approach an interview is understood as a conversational interaction between interviewer and interviewee. For this reason, I include patterns and remarks on the interviewee's accounts both the first and second phase of the interview.

${ }^{13}$ More details on the methodology and methods for the research design, data collection, and analysis process are presented in Chapter III Methods.
} 
I met Anmi for the first time through Ms Kim, a social worker at a care centre in the eastern part of Seoul. When I made contact with her, I had already conducted 15 interviews, so I had worked out several preliminary patterns and characteristics from my material. For the means of a more detailed analysis, I was looking for cases that, at least on the surface, were different from the ones I had so far. Therefore, I asked Ms Kim to find me three FCHs with very different familial backgrounds. The social worker introduced me to three interviewees, Nanyoung (aged 72, wife), Ran (aged 60, unmarried daughter), and Anmi (aged 54, daughter-in-law). I had already conducted interviews with five care-giving daughters-inlaw, but there had been significant differences between them, and I wanted to investigate deeper by interviewing Anmi.

The day of the interview, Ms Kim and I visited Anmi's house in the morning. On our way there, the social worker gave me basic information about her and her family. According to Ms Kim, Anmi had suffered from the care situation and relationship with her mother-inlaw. She used to care for her mother-in-law as a FCH while working part-time, but now her husband had registered with the LTCI as a FCH instead of her since she had been fired from her part-time job and it is not allowed to receive both unemployment benefits and payments for LTC. Anmi's husband also was self-employed as a delivery car driver at the time.

After we arrived at Anmi's house, Ms Kim briefly introduced me and informed Anmi about the interview, then she left. The interview lasted for 2 hours 9 minutes. I also saw the mother-in-law very briefly when I came into the house, but I did not get to talk to her since she immediately retreated to her room and did not show again until I left.

\subsubsection{Anmi’s self-presentation}

This interview was one of the most challenging interviews to conduct in that the interviewee tried not to open some topics but instead brought back a specific topic repeatedly: the hardships in caring for her mother-in-law. Although I expected the interviewee's accounts on social rights as an individual worker, she spent almost all the time of the interview on speaking about the relationship with the mother. When I asked the opening question to generate her main narration, she began her narration about her caregiving by explaining her mother-in-law's daily routine of obsessing about her health conditions: 
"She used to take care of herself, especially her health care, completely. She was well-educated, so she knew a lot and was well-informed. She always cut newspapers for her scrapbook and wrote memos (interviewer: okay). That's one of her daily routines. She is herself very meticulous, and she is too proud; no-one can touch her. All children show unquestioning obedience to her. She had always lived like that. Well, she's very strict in her self-discipline and health care" 14

At first, I did not realise what she tried to say by explaining her mother-in-law's lifestyle and character. Then she continued to talk about the deterioration of her mother-in-law's health, the onset of dementia. In the presentations of the mother's character and health conditions, she tried to describe everything without empathy or affections. So, I assumed that she did not have a good relationship with her mother-in-law and did not want to care for her. After her initial narration, she repeatedly came back to talking about how she suffered from having to care for the mother-in-law. She continued to talk about the relationship with mother and how the mother ignored her during the entire period after Anmi had met her for the first time shortly before the wedding. This behaviour shows that even though Anmi and her mother-in-law do not have a good relationship, her mother-inlaw still seems to play a very central role in Anmi's life. Therefore, I formulated the thematic field: "Care as a desperate attempt of belonging to the husband's family by devoting to the mother-in-law as the first daughter-in-law.”

It became clear that the manner of expressing her emotions by caring for the mother-inlaw. While she repeatedly mentioned the bad relationship with her mother-in-law, she also provided me with many episodes of how she worried about her mother-in-law and how she cared for her with all her heart. This contradictory attitude towards the mother-in-law can be interpreted as the bad relationship not being what Anmi wanted and her continued effort to better it. At this moment, one can imagine that the interviewee might be willing to care for the mother-in-law if her mother-in-law accepted it - and Anmi herself. She showed me her willingness to care for mother-in-law, by using the phrase 'feeling of guilt" repeatedly. In the last part of the interview, there was a section where she talked about volunteer work for the elderly (in the context of Christian duties) in general rather than of emotional

\footnotetext{
14 어머니가요, 당신 본인 몸 관리나, 건강관리는 엄청 철저하셔요. 많이 배우셨어요. 그래서 아시는 것도 많고 지식이 많아요. 그래가지고 늘 이렇게 스크랩하고(응) 적고 메모하고 이런 게 일상화 되어 있어요(네) 그리고 엄청 꼼꼼하시고, 자기에 대해, 자기에 대해서는 완벽하세요. 그리고 자존심이 굉장히 강하세요, 아무도 그 분의 마음을 터치할 수가 없어요. 자식들은 감히 부모라 그러면 그냥 무조건적이예요. 그렇게 사셨던 분이거든요. 그래서 자기 관리를 엄청 철저하게 하시고, 건강관리도 굉장히 잘하셨어요(네) 몸에 좋다고 방송에 나오면 그거 그날 즉시 사다가 드시고(네) 그렇게 하셨는 데(161-166)
} 
interactions with her mother-in-law. I interpret this argumentation as an attempt to legitimize herself as a person who is polite to and respectful of the elderly and by extent even to her mother-in-law, who never accepted her daughter-in-law.

Even though she said the main motivation in caring for mother-in-law was her love for her husband, she mostly limited her life story to her experience of the care work. During the interview, she brought her husband voluntarily, and answered me only briefly, sometimes not more than one short sentence, when I specifically asked questions about the husband. Furthermore, when I asked her for some last remarks at the end of the interview, she mentioned that she wanted her interview to be applied to social policies to support family caregivers. When I think about the interview retrospectively, her last words could be interpreted as that she tried to distance herself from the interview.

\subsubsection{Reconstruction of Anmi's life story}

Anmi represents six interviewees whom I summarise as the type "Traditionalists". These interview participants are similar, in that they have problems in terms of family relationships with the elderly. Anmi (aged 54) has cared as a FCH for her mother-in-law, who has dementia, since 2010. She lives together with her husband, her first daughter, and the mother-in-law. Anmi has two daughters, but the second one married and lives separately. Anmi has lived with her mother-in-law since the early stages of her marriage, but the relationship between them has consistently been poor.

To analyse Anmi's experience of caregiving, I reconstructed her life history within the framework of a female Korean baby boomer's ${ }^{15}$ life-course. The life-course approach makes it possible to conceptualise the dynamics of social change, although one should consider gender cleavage as a key dynamic factor (Kohli 2007: 262). As Yi Hee-Young (2005) has argued, this approach enables researchers to reconstruct social structure through the concrete life history of an individual.

\footnotetext{
${ }^{15}$ The phrase 'baby boom' refers to a noticeable increase in the birth rate. The concept of Korean baby boomers is defined by a total birth rate exceeding 3 per cent. According to this standard, the Korean baby boomers are those who were born between 1955 and 1963. The concept of the Korean baby boomer is often associated with social issues like pension and health care policies. Baby boomers also have a significant effect on politics from the macro perspective.
} 


\section{Family background and childhood}

Anmi was born in a small fishing village in 1961 when Korea was an underdeveloped country and had just started to industrialise. She spent her first years in a typical small countryside village, where most villagers including her parents engaged in small farming. She has three sisters and two brothers and is the fifth of six children. In addition to low levels of economic development, average education levels in Korea were also low; educational opportunities were gendered, and boys typically had significantly better opportunities than girls during Anmi's childhood in the late 1960s and early 1970s. Unlike her brothers, she had to stop her education after her elementary school, like many other girls of the Korean baby boomer generation.

"My older and younger brothers went to university, but we daughters just graduated from elementary school. (Okay, I see), well, I am one of them, too."16

As Jun Hee-Kyung (2012) discusses, while boys were considered as youths who should be educated and protected in South Korea in the 1970s, girls were considered to mature earlier and were, therefore, more likely to enter labour market sooner (Jun Hee-Kyung2012: 49). Anmi's schooling, moreover, could not be financially supported by her family. She felt sorry for herself and felt she would have done well if she had had someone to support her in continuing with school. She wished to study and tried to educate herself whenever she had the chance. This tendency was not unique to Anmi but applied to many other girls in the late 1960s and early 1970s. In this period, Korean girls were not considered deserving of family resource investment as future citizens; rather, the girls played a role as the future mothers who would discipline and care for those ‘future citizens' (Kim Hye-Kyung 2006).

"It was hard for me. I think of the past though, if there was someone for me, now, I learned again though (Interviewer: okay), like math, or like English, when I had someone who could help me deal with some challenges, like math-then I could perform it very well, I think. So, even now, I feel the lack of that education. $" 17$

\footnotetext{
16저는요 오빠나 남동생은 대학을 다 보냈어요. 그런 데 저희 딸들은 다 그냥 초등학교로 졸업을 했어요.(음, 네) 그래서 저도 그 중에 하나예요.(1184)

17힘들었어요. 그런데 지금 생각하면, 누가 나를 좀 이런, 지금 인제 이렇게 공부하면서(네네) 수학 같은 거 이렇게 보면, 영어 같은 거 뭐 이런 것 좀, 누가 수학 이렇게 답답한 거를 좀 가르켜 주는 사람이 있었으면, 내가 이걸 참 잘했을 것 같으다. 그런 생각이 들어서 그런 아쉬움은 지금도 남아 있어요.(1193-1195)
} 
Anmi and her siblings illustrate the gender inequality in education that was very salient at the time. While Anmi's two brothers finished higher education and both graduated university, none of the three sisters and Anmi, got to continue their education after elementary school.

These tendencies remained until the 1980s when girls' high school entrance rates climbed to parity with those of boys. The educational disparity between boys and girls can be understood as a family strategy founded on filial piety based within the Confucian hierarchical system. Boys had always been treated as more important than girls, especially the eldest son, who was considered the most important child as he would take on the family's duties and responsibilities (see Chapter II, section 2.3). In addition to the cultural context, these gendered educational opportunities can be understood as a family's selection and concentration strategy, given that there were not enough resources to educate all children, given the tendency of having many children to make up labour force in agricultural society. In this regard, parents put more resources into the education of the first son, who would take on the family's responsibilities, such as caring for the parents or performing the ancestral rites. This tendency was not just a cultural norm, rather, the rules were established by law until recently. The first son was prioritised for inheritance until 1989: Articles 975 and 977 of the Civil Act in South Korea ensured the right of primogeniture. The Civil Act was amended to provide for the equal distribution of inheritance regardless of gender in 1991, but although the equal distribution was provided for in legal terms, the cultural value of filial piety has remained in practice as a tradition (Namgung Myung-Hee 2010: 9). Anmi’s family has also retained this tradition, which means only Anmi's brothers inherited from the parents, while Anmi and her sisters received nothing. Anmi agrees with this cultural value, referring to a traditional Korean word, 'Chulgaoein (출가외인, 出嫁外人)', which reflects the idea that a married daughter is no longer a member of her original family:

"Still, our way of thinking, in my region, my mom said that daughters were Chulgaoein. Such a way of thinking, still, I agree with (interviewer: okay). We 
are familiar with the idea (interviewer: okay). That's why sons distinguish themselves from their sisters." 18

And Anmi also accepts that in the distribution of the estate only the sons are considered:

"Nobody raised objections to the decision. (interviewer: how come?) Naturally, they are sons. A Son is a son (interviewer: but daughters...) A daughter is Chulgaoein (interviewer: okay). My sisters agree, too (interviewer: ah, do they?). Yes (interviewer: ah). So, a daughter is Chulgaoein. I belong to my husband's family (...). Now, I don't think I have any responsibility for my mom, for my original family (interviewer: ah, okay). I've lived that way until now."19

After Anmi's graduation from elementary school, she moved to Seoul to find a job at the age of 14. Like Anmi, South Korean girls in the 1970s were likely to have a short period of education after which they were to find a manual job in the light industry sector. At that time, young 'obedient' girls from the villages played a pivotal role in economic development in South Korea's export-oriented economy, since they had no choice but to endure long hours of labour in poor working conditions (Jun Hee-Kyung 2012: 50). Anmi followed a friend, and they moved to Seoul together where they could look for a job deemed fitting for girls their age. She found a job in manufacturing and worked there until she got married in 1983 at the age of 22 years. She lived apart from her family in a worker's dormitory, and she started to attend a Protestant church. In the period of industrialisation and urbanisation in Korea in the 1960s and 1970s, young workers started to migrate to the cities. Away from their families, the young workers have no feeling of belonging and Protestant churches t may offer an accessible community to be part of. The religious values Anmi established for herself in this period, helped her to bear the demanding situations she faced during her life-course. The church and religion play a central role in her life to this day.

\footnotetext{
${ }^{18}$ 그렇지는 않는데, 자기네들이 그렇게 생각. 우리 아직도 사상이, 우리 전라도는, 엄마가 딸은 출가외인이라고 그런 사상들이 아직도 우리 저도 그렇게 되고, (네) 그렇게 박혀 있거든요(네) 그래서 그런지 이 아들들도, 누나들은 별개라고 생각해요 (729-731) 
"I still attend that church (interviewer: ah, do you?). I was married there (interviewer: I see). I have spent time at a church." 20

\section{Marriage and first meeting with her mother-in-law}

As mentioned above, Protestantism is a very important value for Anmi. In fact, she met her husband in the church community. When she married in 1983, she met her mother-in-law for the first time, and she realised that marriage and organising a family were very practical issues in terms of economic situations and relationships with her family-in-law. Her husband was the first-born son, with two younger sisters and one younger brother. At that time, her husband lived with all of his siblings and his mother. His father, who was a naval officer, died when he was young. Since the father died in his early age, his family was not entitled to pension benefits for the bereaved family. Anmi heard that his family had been affluent before the father had died, but experienced severe economic difficulties and decline after the loss of the main earner. Nevertheless, the mother and the family refused to adjust their lifestyle to their new economic and social status.

At the time of his marriage to Anmi, her husband lived with his family in a house, the deposit and rent for which were paid by the elder of his two sisters (the second-born child). This sister, however, did not want to live with her brother and his new wife so the rest of the family had to find a new place. But since Anmi’s husband had not saved enough money to rent a house of his own, there ensued a conflict between the family members. Anmi seems embarrassed when speaking about memories from that time:

"I had no idea about maintaining a household, because, at home, I had lived in my mother's house, then moved to Seoul alone, where I lived in a dorm (interviewer: okay). I had known only about my mother's house, where I had lived. ... I had no idea about housing and its costs (interviewer: ah). I had lived only in a dorm." 21

Anmi was very frustrated due to the economic situation within, and relationships with her new family.

\footnotetext{
20그 교회를 지금까지 다니고 있어요?(아, 그래요?) 그 교회에서 결혼을 하고. (그러시구나) 그래서 교회하고 저하고 같이 세월이 흘러갔어요.(92-93)

21그런 데 저는요. 집에 대한 그런 개념이 하나도 없었어요. 왜냐하면 집에서 엄마 집에서 살다가 서울 떨렁 올라와 가지고는 기숙사에서만 살다가(네네) 이렇게 엄마 집만 기억을 하지, 엄마 집에서는 내가 늘 있던 집이니깐, 그래서 결혼하고 방을 얻고 돈이 얼마 그런 거를 전혀 몰랐어요(아) 기숙사에서만 있어가지고 (1256-1259)
} 
According to Anmi, her husband did not earn enough money to maintain a household and a family of his own because he had to financially support his mother after his father's death. Anmi’s mother-in-law firmly requested to live separately from Anmi and her husband, even though the family's budget did not allow it. Anmi had expected to live with her mother-inlaw, whom she expected would help her with domestic work and caring for the children. Her mother-in-law refused this, however, and Anmi accepted the decision since she had less power in her 'new family' as a daughter-in-law. She finally looked for a separate flat for her mother-in-law:

\section{"I could not speak (couldn't?) yes, I couldn't. When I realised that, I had already fixed the date for the wedding. So, it was no use saying that. I cried a lot, and even almost went mad. At that time, I felt that this situation nearly sent me crazy." 22}

There is a long history of conflict between Anmi and her mother-in-law. Since Anmi met her, she has never had positive experiences with her. Anmi assumes the following:

"She was different. That's why (interviewer: okay) she did not try to teach her children; her role as a mother just stopped after the death of my father-in-law. I heard that she lived in affluence before." 23

Anmi asked her mother-in-law to care for her children, but her requests were rejected. Instead, her mother-in-law wanted to live with a friend and did so. After five or six years, however, she came back to Anmi and her husband's house. Anmi mentioned that her mother-in-law came back because her granddaughters had grown old enough that she did not need to care for them anymore:

"At that time, my children had already grown up. They were maybe six, maybe seven (yes) when my mother-in-law came back (yes), so, since then, I rented a room by the month. I had to rent a two-room flat, and it was difficult (yes).

\footnotetext{
22이야기를 못했죠(차마?) 네, 이야기를 못했죠. 어차피 결혼날짜를 다 잡아놓고 그걸 알았는 데 그때사 이야기하면 뭐 어떻게 하겠어. 제가 많이 울었죠. 울고 정말로 정신이 돌 정도로 내가, 아 이렇 게 되며는 내가 미치겠구나 하는 거를 그때 느꼈어요. (1270-1272) 
Anyway, I rented a two-room flat and started doing that when my children were about five or six years old." 24

"She used to take care of herself, especially her health care, completely. She was well-educated, so she knew a lot and was well-informed. She always cut newspapers for her scrapbook and wrote memos (interviewer: okay). That's one of her daily routines. She is herself very meticulous, and she is too proud; no-one can touch her. All children show unquestioning obedience to her. She had always lived like that. Well, she's very strict in her self-discipline and health care (interviewer: okay). Whenever she saw information about healthy food on television, she would buy it immediately and eat it (interviewer: okay); she did so.” 25

According to Anmi, her mother-in-law did not share or help with household chores or caring for the granddaughters at all. Anmi even mentions an example of the mother-in-law neglecting her granddaughters. Even though Anmi never felt close to her mother-in-law, to begin with, the relationship between the two women grew increasingly worse:

"She did not open her mind, even when we lived together. She bought her items separately, put all the stuff in her room, and every morning she took her personal washing tools, such as a toothbrush, a soap bar, or towels, out of her room, even to bathe."26

"I wanted to talk with my mother-in-law and recover our relationship, but she said 'How dare you? You should not touch my life; I do my things so that you don't need to care about me. Mind your own family', like that. I wanted to ask why she spoke like that; we were also family members together. I wanted to argue with her to improve our relations, but I couldn't (interviewer: ah). She snapped the door of her mind, and such situations occurred repeatedly, I had no good feelings towards her."27

\footnotetext{
24이제 그때는 아이들이 벌서 컸어요. 뭐 여섯, 일곱살 됐지 뭐 (네네) 그 세월이 흘렀으니깐(네네) 그래가지고 그때부터서 이제 방을 이제 월세를 또 얻었어요. 방을 두 개를 얻으려니, 힘들잖아요(네) 그래가지고 월세를 얻어서 인제 그렇게 시작하게 됐어요. 애들은 한 다섯 살 여섯 살 때부터(152-154)

${ }^{25}$ 어머니가요, 당신 본인 몸 관리나, 건강관리는 엄청 철저하셔요. 많이 배우셨어요. 그래서 아시는 것도 많고 지식이 많아요. 그래가지고 늘 이렇게 스크랩하고(응) 적고 메모하고 이런 게 일상화 되어 있어요(네) 그리고 엄청 꼼꼼하시고, 자기에 대해, 자기에 대해서는 완벽하세요. 그리고 자존심이 굉장히 강하세요, 아무도 그 분의 마음을 터치할 수가 없어요. 자식들은 감히 부모라 그러면 그냥 무조건적이예요. 그렇게 사셨던 분이거든요. 그래서 자기 관리를 엄청 철저하게 하시고, 건강관리도 광장히 잘하셨어요(네) 몸에 좋다고 방송에 나오면 그거 그날 즉시 사다가 드시고(네) 그렇게 하셨는데(161-166)

26마음의 문을 안여셨어요. 같이 살아도요, 당신 쓰실 물건 다 따로따로 사놓고 방에 갖다놓고, 아침마다 세수하면 당신 칫솔 당신 쓰실 뭐 세수비누 타올 다 갖고 다니면서 쓰셨어요. (166-167)

27제가 그, 근데 그걸 어머니한테 말을 해서 풀고 싶은데, 감히 어머니가, 내 생활에 터치하지 말라, 나는 내 마음대로 사니깐, 내 생활에 터치하지 말고, 너네들끼리만 살아라, 이런 식이니깐. 왜 이러세요. 가족으로서 왜 이러세요. 이렇게 저는
} 
Anmi's mother-in-law did not try to create a basic emotional exchange or empathy between herself and other family members, and this made it impossible to establish a family bond.

"If the elderly stay out a long time, or if it rains or snows, one should care about them and call them (interviewer: I see). I think one should do so as a family, but my mother-in-law replied when I called, 'Why did you call me? Did you try to fetch me over? You never need to call me; when something happens, I will call you'-she told me so flatly, no communication between us, and she repeated it over and over. It hurts me, even if it's small things. So there are a lot of hard feelings, even if there are no fierce fights or quarrels in the house. We keep calm outwardly, but there are huge inner conflicts." 28

"She cared for her daughters' children when she visited them. If her daughters asked for her to care for the children because they were absent, then my mother-in-law stayed there and cared for them, even for several days. If her daughters wanted their mother's help for some family affair, then she was there and helped with everything." 29

To deal with her difficult situation, Anmi turned to religion:

"If I had not been a religious person, I could never have endured my mother-inlaw. I must have divorced, for my husband absolutely respects his mother (interviewer: okay). Even now, he is happy to see his mother day and night (interviewer: I see). As a son, well, he is such a person. I could not have stood that, and my family would have been ruined without my religious faith. "30

\section{The on-off career in paid work}

After she got married, Anmi had to take care of her children without the help of a public childcare system. In this situation, she could not maintain a steady working career. Instead, she had to enter and exit the labour market repeatedly. She stopped working when her

\footnotetext{
오목조목 따져 가면서, 싸워 가면서, 그 감정을 풀면 좋은데, 그게 안돼요. (아) 탁 닫아 버리니깐, 그게 계속 쌓여 있으니깐, 좋은 감정이 없어요. (347-350)

28노인이 밖에 나가서 시간이 안되고, 비가 오거나 눈이 오면 걱정이 되서 전화할 수 있잖아요(그럼요) 당연히 그게 가족으로서 해야 돼요. 그런 데 우리 어머니는 전화 하며는 '니가 날 데리러 올라고 그러냐, 왜 전화하냐. 무슨 일이 있으면 어련히 연락을 할까봐 그러냐’ 이런 식으로 딱 잘라 버리니깐, 이게 소통이 안되니깐, 그 감정이 쌓이니까는 이제 작은 것 까지도 쌓여요. 그래서 그런 감정들이 많으니깐, 집안이 시끄럽고 막 그러지는 않지만, 평온하지만, 그 내면에 속에 쌓여있는 감정의 골이 너무 깊은 거예요. (353-357)

${ }^{29}$ 가시며는 다 봐주세요. 그 딸이 엄마 나 어디 갈꺼야 애기좀 봐줘 그러면 며칠이고 다 봐주세요. 그리고 뭐 딸 집에 뭐 엄마 나 집 뭔 잔치있어, 그러면 가서 다 해주세요(559-560)

30 제가 마음에 종교가 없었으면, 제가 우리 어머니를 지금까지 제가 이렇게 쌓여잇는 것을 품고 참고 살아오지를 못했을 것 같아요. 아마 이혼을 했던지, 우리 남편은 절 대적으로 어머니(응) 지금도 아침저녁으로 보는 것만 어머니 얼굴 보는 것만도 행복해 하시잖아요. (네네) 아들은. 그러니깐 그런 분인데, 내가 내마음 속에 종교가 없었으며는 가정이 유지되지 않았을 거예요. (996-999)
} 
children were born. Later, she got a part-time job and opened a small grocery store when the children were old enough. Her discontinuous career shows that a woman's life-course in paid work is bound up with her life-course in the domestic arena. While she has only been able to work on and off due to her responsibilities of caring for their children and later her mother-in-law, her husband has constantly worked for a living. At first, he worked at a small factory, then he became a driver, transporting goods with his own small truck. He started to pay contributions to the national pension system (NPS) as a voluntarily insured in the 1990s.

Hwang Jung-Mi (2007) argues that women's social rights depend on their status in both the paid labour market and the family. Social rights as a worker are very important to be eligible to receive a public pension. This, however, requires an individual to be consistently employed and pay the contributions for the social insurance system Thus, interruptions caused by caring for children to exclude women from individual entitlement to pension benefits. Such a discontinuous work biography also hinders women in their career path and possible professional advancement, since they can only obtain a precarious livelihood. Anmi has also experienced career interruptions due to childcare and re-entrance into the paid market. She ran a grocery shop for nine years once her children were old enough to allow this. However, she was always longing for self-improvement and mentioned her resolutions relating to her career:

"When I left my grocery shop, there were so many things I wanted to do to challenge myself, but when I ran my shop, I had to be there at 9 or $10 \mathrm{am}$. Sometimes, I had already visited a wholesale market, even at dawn, and then I had to stay in the shop until it closed at $10 \mathrm{pm}$. I had no time for myself." 31

In 2010, Anmi finally decided to shut down her shop to take a rest and improve herself. One of the things she achieved during this year, was obtaining a care-helper licence:

"Even if I shut down my shop, I had to do something for a living again. I could not afford to stay at home and be well off without a paid job (interviewer: okay). I, therefore, did everything I wanted when I took a year off work (interviewer: okay). I got a cooking licence (interviewer: okay), and this one [a care helper licence]. At that moment, I never thought my mother-in-law would get dementia

31네네네(네) 그렇게 시작 해가지고, 이제 반찬가게를 접으면서 제가 (네) 하고 싶은게 참 많았는데, 아침에 10 시, 9 시에 출, 새벽에 시장갈 때는 새벽에 가서 10 시에 문을 닫고 오며는, 제 시간이 하나도 없는 거예요 (333-334) 
though (Interviewer: yes); but if, for example, in time my mother-in-law got dementia, in order not to hate her (interviewer: okay), well, I would hate her less if I got paid like that (interviewer: yes). I have had bitter feelings about her for a long time since she secluded herself in family relations. I really cannot understand her hiding from me." 32

In this regard, it is hard to understand Anmi's caregiving in the context of a mere family obligation. The reasons behind her becoming a FCH are a mixture of her desires for selfdevelopment and future planning, offsetting complicated emotions like hatred and guilt. Especially, caring for her mother as a FCH was not derived from the family obligation. Rather, Anmi considers the payments she receives for caring for her mother-in-law as compensation for performing unwanted caregiving. Anmi has had a poor relationship with her mother-in-law all the time since her marriage so she could not care for her mother-inlaw without rewards or compensations.

\section{Anmi's qualification as a care helper}

By Anmi’s account, her qualification was motivated primarily by thoughts about her future life and employment career. Even if she obtained the care helper licence partly with family care in mind, her caregiving was intended for her husband rather than for her mother-inlaw:

\footnotetext{
"Well, I had only one year to take a rest, then I would have no free time when I came back to work; so I decided to do something while I took a rest, and I did, when I thought that, for example, if something happened to my husband in the future, I could care for him (interviewer: I see). Also, I heard that the job has no common retirement age (interviewer: okay); so, yes, for me, actually, as an occupation; instead of caring for mother, I will continue that work even though I become old, this is in my mind, this was my intention (interviewer: umm yes). That's why I got the licence; well, something happened to the mom. A year after I got the licence, she became like this."33
}

\footnotetext{
32네, 그래가지고 그걸 하고 이제 접으면서, 제가 이제 그 또 직장을 그걸 접으면서, 또 직장을 다녀야지 제가, 집에서 이렇게 여유롭게 지낼 수 있는 경제적인 여력이 안되니깐(네) 제가 1 년동안 쉬면서, 제가 하고 싶은 걸 다 했어요. (네) 그래서 뭐 조리사 자격증도 따고, (네) 이것도 제가 우리 어머니가 이제 그때까지만 해도 치 매라고는 생각을 안했죠(네) 근데 만약에 예를 오래 되가지고, 예를 들어 우리 어머니가 치 매가 걸리며는, 제가 우리 어머니 안미 워하려고(네) 그래도 내가 이렇게 수입이 있으면서 하며는 좀 미움이 덜할 것 같아요.(네네) 그 전에 쌓여 있는 것, 혼자서 은둔생활 하면서 그 쌓여있는 감정이 가족으로서 도저히 이해할 수 없는 관계들이 막 쌓여가지고 (340-345)

33네가 그래서 이제, 그 어차피 제가 1 년만 쉬기로 작정을 했기 때문에(네) 1 년 후에는 또 직업을 가지며는 제가 자유가 없기 때문에, 그 동안에 이제 하고싶은 거를 해야 되겠다 해서 이제 한 게, 그리고 그거를 따놓으며는, 예를 들면 우리 남편이 나중에 그런 일이 있으며는, 또 제가 보호를 할 수가 있고(그럼요) 또 그 다음에 이제 제가 직업으로서 그게 정년이 없다고
} 
Anmi thought to care for old people was not only a job with a good outlook (no common retirement age) but also felt the care work itself to be worthy in terms of religious piety and as a social activity:

"I would like to respect for the elderly, I couldn't do that for my mother-in-law. I wanted to do it for other old people, based on my religious convictions (interviewer: okay), mainly my religious conviction. I find it is worthwhile, and my husband thinks so, even now."34

\section{The mother-in-law's dementia and her refusal to be diagnosed}

The mother-in-law had continuously refused to visit a hospital to be diagnosed with dementia between 2010 and 2012. She feared she might be forced to enter a care facility if she was diagnosed. As time went by, her symptoms got worse and worse, until Anmi could not bear it anymore. She says that she tried, again and again, to get her mother-in-law to visit a hospital in order to be diagnosed, without any success.

When her mother-in-law was finally assessed for using the LTC services, she however only scored Grade 3, which did not cover facility care services. Prior to this Anmi had hoped her mother-in-law could be put in a nursing home. Apparently, it was already hard to get a diagnosis placing her in Grade 3 of the system, since her symptoms appeared randomly, which made it difficult for the assessors from the national health insurance service to get a picture of the full extent of her illness.

"I think the assessment system for grading is ridiculous. Indeed, we could not bear it (interviewer: painful?), it was too hard to live, but they visited us, and she did not poop at the very moment they visited. I'd washed them away. How can I invite people to such a dirty, stinky house? (...) I had bathed my motherin-law before the assessors came, then they came and just asked her questions about the value of coins or her family members. When they questioned her, she replied properly at that time (interviewer: okay). It didn't last, though; she suddenly changed, up and down. It was too much for me. So, I called the assessment centre for entitlement to the LTC services, saying, 'How can we

그러더라구요(맞아요) 네, 그래서 제가 이제 사실은 이제 어머니에 대한 것보다, 인제 직업으로 나중에, 내 노후에 내가 그걸 그 일을 해야 되겠다는 이제 그런, 생각으로, 그 생각이 더 컸죠. (음,네) 그래서 이제 그걸 따 놨는데, 이제 어머니가 이렇 게 되버린 거야. 그거 따고 일 년 됐는데, 어머니가 이렇게 되버린거야 (391-397)

${ }^{34}$ 내가 노인을 이렇게 섬기는 그런 마음, 내가 우리 어머니는 감정이 쌓여서 그렇게 못하지만, 내가 그거를 다른 노인들한테 이렇게 하고 싶다, 그런 신앙에서도 있었고(네네) 신앙심이 더 많이 작동을 했죠. 그게 보람된 일이라고 생각을 하고, 우리 남편도 그 생각을 갖고, 지금고 갖고 있어요(415-417) 
protect her? How can we solve the problem? She often comes out of the house and loses her way home, and the police find her, but you do not permit her the right Grade for using the LTC services. What can we do? How can we do it?' However, they had their own standards on grading; she needed to meet those standards." 35

After repeated failure to secure proper assessment, Anmi got a surveillance camera installed to prove her mother-in-law’s symptoms.

"Well, later, I got totally upset (interviewer: yes), so I used a camera to take videos. At night, I recorded at 3 am in the morning (interviewer: ah). Well, I showed them everything." 36

Although the mother-in-law had emotionally closed off to her daughter-in-law, she had to depend on the Anmi's help when she got dementia, as she could not live independently. She needed help not only with the instrumental activities of daily living (IADL) like cleaning, maintaining the house, and preparing meals but also in some activities of daily living (ADL) like bathing and hygiene. Anmi especially found caring for her mother-inlaw demanding due to her impaired cognitive functions. She also repeatedly expresses her frustration with failing to form emotional bonds with her mother-in-law, in feeling compassion for her and in fulfilling her duty as a daughter-in-law:

"I have to pity her, but I always envied her, because she always had better things than I did; I had to save, cut and budget every day. She enjoyed everything I

\footnotetext{
35 오셔서 그 분들이 그 등급 먹이는 게 너무 웃겨요. 정말 우리는 너무(괴로운데) 힘들어서 살 수가 없는데, 그 분들은 와가지고, 그때만, 그때 똥을 싸고 그러지는 않잖아요. 다 씻겨, 사람이 온다는데 더럽게 해놓고 있을 수, 냄새나게 해놓고 손님을 받을 수가 없어요 제가(그쵸그쵸) 그러니깐 다 닦여놓고 깨끗이 해놓고, 그러고 정신이 인제 누가 오면 멀쩡해요. 맨날 그 흐렷을 때 와야 하는데, 그거 맞추기가 힘드. (오락가락 하시니깐)그 분들도 시간 맞춰갖고 자기네들 스케쥴에 맞춰서 오니깐, 내가 이랬을 때 오시오하며는 그때 오면 정말 좋겠어요. 그런 데 그게 아니예요. 자기시간에 맞추니깐. 어머니는 다 씻겨놓고 깨끗해져갖고 있는데, 그때 오셔가지고 뭐, 동전이 얼마냐, 가족이 누구냐 이렇 게 물어 보며는, 대답을 한다구요. 그때는 또(그쵸) 그게 계속 지속이 되는 게 아니고, 갑자기 돌변해갖고 막 그러다가, 또 멀쩡하잖아요. 그게 너무 힘든 거예요. 그래서 제가 막, 그래서 요양 센터에, 등급센터에 전화를 해가지고, 그러며는 우리들은 어떻게 보호를, 어떻게 우리들은 해결을 해야되냐. 집에서 막 문을 열고 나가셔가지고, 집도 잃어버리고, 막 경찰서에서 막 찾아주고 이런 상황이 되는데, 등급을 안먹여주며는, 우리가 어떻게 하냐, 어떻게 해야되냐. 근 데 자기네들은 그 등급에 기준이 있으니깐, 거기를 넘어가야지 되잖아요 (294-305)

36네, 나중에는 너무 화가 나가지고(네) 제가 이제 그 상황들을 이렇게 영상으로 찍어놨어요. 밤에, 밤, 시간도 찍어놨어요. 밤 3 시(아) 그래 그걸 다 보여줬어요. (314-315)
} 
wanted, so, uh, the feelings towards my mother-in-law, I couldn't feel love for her. ${ }^{37}$

\section{The only one responsible for unaccepted responsibilities}

Anmi said the most difficult thing in caring for her mother-in-law was the lack of communication and kinship. Anmi respects the elderly; she tried to show this respect and get close to her mother-in-law, but Anmi failed to connect emotionally with her. This experience had an influence on her caregiving. In the interview, she repeatedly mentioned her feelings of guilt.

"I am living in a world of guilt. I want to fulfil my duty as a daughter-in-law, but she does not receive it but refuses it. I gave that up now; as a daughter-in-law, I should show respect for the elderly and serve them, but she refuses even that, and I always feel guilty." 38

Even with the relationship between the two continuously deteriorating, there is no choice but to live together. Anmi said her mother-in-law did emphatically not want to move into a nursing home, and yet she could also not live with any of her other adult children, because the daughters thought the eldest son should take his responsibility of caring for a parent. At first, Anmi intended to put the mother-in-law into a nursing home; she had applied for her mother-in-law's entitlement to LTC services especially for care facility services, rather than for in-home care services. However, she eventually took care of her mother-in-law at home as a FCH for several reasons. Firstly, her mother-in-law wanted to stay at home and feared to enter a nursing home.

"So, at home alone, she said she liked it here, staying at home alone, opening the fridge, and cooking and eating by herself, as she does." 39

\footnotetext{
37불쌍한 마음이 들어야 하는 데, 늘 나보다 좋은 건, 저는 이거 일구기 위해서 맨날 아끼고 절약하고 이렇게 살았는데, 우리 어머니는 맨날 나의 부러움이었거든요. 내가 하고싶은 걸 다 하시면서 사신 분이고, 그래서 어, 어머니에 대한 감정이, 그렇게 이렇게 사랑하고 싶은 마음이 안들어요. (1033-1035)

38그리고 제가 죄책감에 살구요. 내가 당연히 해야 할 도리인 데, 그걸 안받고 거부를 하니깐, 내가 할 수가 없으니깐, 이제 그걸 체념하고 사니깐, 내가 며느리로서, 어른을 대접해야 하고, 섬겨야 하는 데, 그 일 까지도 거부를 하니깐, 제가 늘 죄책감이 드는 거예요. (359-361)

39 그래서 그냥 집에 혼자 당신은 여기가 좋대요. 나 혼자 이렇게 있으면서, 집에서 막 냉장고 다 해갖고 먹을 것 다 혼자 드시고, 이렇게 하셔요 (470-471)
} 
In South Korea, many elderly have negative associations with moving into a nursing home away from their adult children (Shin Kyung-Ah 2011). To avoid entering a care facility, the mother-in-law did not want to admit her symptoms, even now.

As mentioned above, Anmi had no power to make decisions regarding the care settlement for her mother-in-law. Anmi mentioned that she did never want to care for her mother-inlaw because of the bad relationship between them. However, she also mentioned being aware of her duty as the oldest daughter-in-law.

Besides, Anmi had an overall positive image of LTC facilities, so she thought it would be better for her mother-in-law to enter a care facility rather than being cared for by family members who she is in conflict with. Indeed, Anmi's mother had moved to a LTCI facility after her husband, Anmi's father, had died and the mother could no longer live independently. However, Anmi’s mother wanted to stay at her home and initially resisted moving into an LTCI facility, insisting that care responsibilities originally lay with her children:

\begin{abstract}
"Mom tried never to enter (interviewer: at first) she had lived there all the time (interviewer: okay), she was, but she decided to enter there so that her children looked for the LTCI facility for her and she went there, but, she cried a lot when she went there by saying 'I have many children, but how come should I go there?', she cried a lot."40
\end{abstract}

Not only Anmi's mother resisted the idea of living in a care facility, Anmi's sisters too did not like the idea of their mother going there; rather they expected the first sister-in-law (the wife of their oldest brother) to take on the care responsibilities for their mother. However, Anmi sympathised with the reservations of her sister-in-law so that she persuaded her other sisters to put the mother in the LTC facility. Anmi describes that her mother later started to have a more positive view of living in a facility, especially experiencing the support the staff provided as very convenient and helpful. This change in the mother's point of view set Anmi at ease:

\footnotetext{
40어머니는 안가시려고 (처음에는) 그 평생을 살아온 집을 못떠나지요(네네) 그랬는 데, 나중에는 이제 가신다고 그래갖고 이제 자식들이 알아봐가지고 넣었는데, 그렇 게 가시게 되었는데, 가실 때는 엄청 우시고 내가 자식이 몇 명인데, 거기를 가야.

그러면서 엄청 우셨어요.(671-673)
} 
"Mother said that no one was better than staffs in the centre (interviewer: ah, okay) she had lived alone, so she had known how difficult it is to live alone. But they prepared a meal, snacks, and there was a song class there, laundry, bathing, enough and good foods. Therefore, the mother said one could stay, and my aunt also stays at the facility." 41

The episode on Anmi's mother shows that Anmi had a positive view on LTC facilities and she made her voice heard in the negotiations on the form of care her mother should receive. Anmi's other sisters expected the first sister-in-law to take on the care responsibilities based on the filial piety, but she objected to taking on the burdens of being a caretaker at home, and finally, other family members, including the mother herself, agreed with that. It should be noted that the form of care the elderly receive, depends on either a decision of the elderly themselves or negotiations between two (or more) individuals who directly relate to each other; caregiver and care-receiver. So, decision making on care settings for the elderly depends heavily on dynamics between family members. Other family members' expectations make Anmi care for her mother-in-law. Especially, Anmi's husband as the first son of the family valued filial piety to his mother and the first son's obligations above anything else, and he asked Anmi to care for and to respect his mother. Even if she was far from having a good relationship with her mother-in-law, Anmi accepted the request of her husband to make him happy.

In addition to that request of Anmi's husband, her sisters-in-law (the sisters of Anmi's husband) apparently also repeatedly asked Anmi to care for Anmi’s mother-in-law. Anmi has two sisters-in-law, whom she says had a good relationship with and often visited their mother. With this in mind, I asked Anmi why her sisters-in-law did not care for their mother at their home instead of her. She explained that while the sisters-in-law loved their biological mother, they did not want to commit to caring responsibilities. Anmi speaks of instances in which she asked one of her sisters-in-law to care for her mother-in-law for short periods of time since the sister-in-law worked at a school as a cook so that she had a vacation in summer. At first, the sister-in-law took the mother home and had her stay with her for two or three days, but she then requested Anmi to take her mother-in-law back

\footnotetext{
41 누가 나를 이렇게 해주냐 그래요(아,네) 혼자서 살아봐가지고 그 힘든 걸 아셨기 때문에, 거기 가니깐 간식 주지 노래선생 오지 빨래 다해주지, 목욕 시켜 주지,먹을 거 그냥 아주 풍성하게 해서 주지 그러니까는 엄마는 늘 너네들도 여기 와서,

그리고 인제 시골에 작은엄마도 계세요. 여기 오라고 좋다고, 이제 그런데 거기는, 제가 여기 보니까는요. (659-662)
} 
home, since she herself rather wanted to enjoy her vacation than help Anmi with her care responsibilities.

As she had to take care for her mother-in-law mostly on her own, Anmi had to transfer her employment status from full-time to part-time. She asserted that she could no longer bear the care burdens after a while because the part-time job offered too little of an income. The other family members, however, ignored the objections she allegedly made every year. Instead of helping her with care responsibilities or agreeing the mother-in-law should be moved to a care facility, her sisters-in-law suggested a certain amount of money as compensation for her decline in income. Although they came to a compromise, Anmi thought the money was still too little in relation to the care work she provided.

In addition to the money from Anmi's sisters-in-law, she, of course, received her wages as a FCH from the LTCI programme. I asked who had the rights to the payments. Anmi replied that the payments belonged to herself since they could only be received for the LTC services offered by a qualified care helper who had obtained a care helper licence and had signed a contract with a care centre in the LTCI scheme. Anmi maintained that there could be no compensations for family care without her qualification and that the other family members agreed with this idea. Anmi received about 170,000 KRW (170 USD) monthly for offering in-home care services as a FCH. The payments are calculated according to care hours offered by a care-helper, and there are no differences in hourly rates between a family care helper and non-familial care helper. However, as mentioned earlier, FCHs can only claim financial compensation for a maximum of one hour per day and twenty days per month, compared to a maximum of four hours per day for a whole month are accepted for the non-familial care helper. In practice, a primary caregiver, however, spends a lot more than one hour daily on care, so there are major gaps between hours recognised by the LTC programme and actual hours offered by the FCHs.

\section{Plans for future: public pension benefit and care}

Unlike her mother-in-law, Anmi says she does not want to rely on her children to care for her in old age. She states that she herself would prefer to enter a care facility as not to impose heavy care burdens onto their children. She also expects living conditions in care facilities to improve until she and her husband might be in need of care. Furthermore, Anmi has prepared for retirement by paying contributions for the NPS (national pension scheme) for more than 20 years. The public pension system in Korea was introduced in the late 
1980s, but the coverage of the public pension was relatively low so that Anmi's and her husband's decision to contribute to the NPS was not very common at that time. Anmi explained their reasoning behind the decision was influenced by the fact they had only girls, which means they have no son to support them when they will reach their retirement age. It should be noted, that the traditional care culture made them plan for their future in nontraditional ways.

\subsubsection{Structural features summary}

Anmi was born in a small village in 1961. She is the fifth child out of six siblings and has three sisters and two brothers. Her parents were farmers in their home village. She had little education possibilities as a girl in a rural area with commonly underdeveloped (not only) educational infrastructure in the Korea of the 1960s and 1970s. While she and her sisters graduated only elementary school, both her brothers got university degrees. A few years after her graduation from elementary school, Anmi decided to move to a big city to look for a job. At that time, her two older sisters left their hometown before Anmi, the oldest one got married and the second sister went to a big city to work at a textile factory. In 1975, when Anmi was 14 years old, she moved to Seoul to find a job in the light industry sector with the encouragement of her friend who had already moved there and succeeded in finding a job at a factory. She started to work as a manual worker in a factory and stayed at dormitory offered by the employer. She continued to work for the same company until she got married in 1983. She saved her salary regularly for her future, marriage and independent living arrangements. She first started to attend a Protestant church when she moved to Seoul, and there, five years later in 1980, she met her future husband who was six years older than her (born in 1955).

After three years of relationship, the two decided to get married in 1983. Right before her marriage, she met his family for the first time. Her husband was the oldest son of four siblings and had two younger sisters and one younger brother. At that time, her husband lived with all of his siblings and his mother. His father, who had been a naval officer, had died when Anmi's husband had still been young. Since the father died in his early age, his family was not entitled to the pension benefits for the bereaved family. Anmi heard that his family had been affluent before the father had died, but after his death, the family the families' status declined. Nevertheless, the mother and the family did not adjust their lifestyle to their new economic and social status. Especially the mother has remained her 
upscale lifestyle until the present day. Due to the economic difficulties, however, the family could not afford higher education for the children. Anmi realized the economic status of the boyfriend's family. After Anmi and her husband got married, his family expected the newly-weds to find a place to live on their own. As the husband had to maintain the household, he had not managed to save any money for his marriage and future life. Therefore, the initial costs for their marriage, including the rent for the house, had to be paid by Anmi. She even had to pay her future mother-in-law's rent for a room, because the mother-in-law did not want to live together with Anmi and her husband. She gave birth to two daughters in 1985 and in 1986, making them a family of four. After a few years, in the early 1990s, her mother-in-law moved back in with them. The children were about six and seven years old respectively at the time.

While Anmi has been working on and off in the course of her life, due to caring for their children and her mother-in-law, her husband has constantly worked for a living. At first, he was employed at a small factory, then he became a driver and transported goods with his own small truck. He started to pay contributions to the national pension system (NPS) as a voluntarily insured in the 1990s. As Anmi's husband was low-educated and earned a low income, Anmi had to work as a secondary earner, who was on and off work according to the family care issues. She stayed at home to raise her two daughters. When the daughters grew old enough to go to school, she picked up a full-time job again. Like her husband, Anmi was also low-educated and therefore paid low wages. In 1998, she started up a small grocery store when her daughters had grown up and did not need her care anymore. She opened the store at 9 am and worked until 10 pm every day. When she needed to buy goods for the store in the retail market, she had to come to work even earlier in the morning. In late 2000, she first started to pay contributions for the NPS as a statutory insured, when the eligibility of the NPS was extended to include self-employed workers. She closed her small business in 2009 due to the too demanding working hours and took a one-year break until 2010. During this period, she got several licences, including a cooking licence and a care helper licence. Since it was hard to support their household on the lone-income of her husband, Anmi could not take a break for longer than one year and went back to work fulltime.

In 2010, Anmi realised for the first time that her mother-in-law was not in a good condition regarding her mentality. At first, Anmi thought the mother-in-law had some kind of mental 
disorder, later, she assumed that it might be symptoms of dementia. As her mother-in-law's abnormal behaviour got worse and worse, Anmi urged her mother-in-law to go to the hospital to be assessed for cognitive impairment disorders. Nevertheless, her mother-inlaw continuously refused to be diagnosed, but repeatedly maintained that she was just suffering from malnutrition due to her missing teeth. Instead of the diagnosis, her motherin-law asked for supplementary health foods. After intensive and repeated recommendations of Anmi's sisters-in-law (sisters of Anmi's husband), the mother-in-law finally went to see a doctor and was diagnosed with senile dementia in 2012. Since then, Anmi applied for the assessment of her mother-in-law in order to become a recipient of LTCI services. However, as a person with cognitive impairments, her mother's symptoms appeared only intermittently and could not be observed by the assessors of the LTCI to their full extent. Accordingly, Anmi set up a surveillance camera at home and recorded her mother's abnormal behaviour in the late night, and her mother-in-law was assessed as a recipient with Grade 3. While a recipient with Grade 1 and 2 can choose all kinds of service settings, including facility care services, short-term facility care services, day care services, and in-home care services, a recipient with Grade 3, 4, or 5 cannot choose the facility care choice. Anmi had to scale down her job to part-time in order to care for her mother-in-law at home since 2013.

Concerning family configurations, Anmi's husband always follows and respects his mother. The mother also loves her oldest son, even showing obsessive behaviour when getting older. While the son and mother have always had a good relationship, the mother has never opened her mind to Anmi. Anmi and her husband are in a good relationship though, care for the mother and domestic work are totally in charge of Anmi, and she took it as her duty. While her husband's siblings (two sisters and one brother all maintain a good relationship with their mother, none of them wanted to care for her. Instead, each of them transferred 100,000 KRW (about 100 USD) monthly to Anmi. Apart from the 300,000 KRW (about 300 USD) from the siblings, the mother received 200,000 KRW (about 200 USD) monthly as benefits from the basic pension (BP), which is a tax-based pension programme. She did, however, not receive benefits from a contribution-based pension system like the NPS or from the pension programme for the bereaved family members of the military officials. The 300,000 KRW (about 300 USD) from the siblings were spent on the mother-in-law's pocket money, while the money from the BP was usually used to cover medications and health costs. The about 170,000 KRW (about 170 USD) Anmi received 
monthly for her care work in the scheme of the LTC programme, was used to maintain cost for all family members.

In 2014, Anmi's second daughter married and left Anmi's house. Since then, Anmi, her husband, their oldest daughter, and the mother-in-law have lived together in one household. In 2015, her husband also obtained a care helper licence, plus, having reached the age of 60 , started to receive old-age pension benefits from the NPS, which amount to about 500,000 KWR (about 500 USD) per month. After Anmi was fired from her part-time job she spent her time caring for her mother-in-law entirely. To receive unemployment benefits from the employment insurance, her husband registered as a FCH instead of Anmi. However, nothing has changed in the actual caring situation, where Anmi is still solely responsible to care for her mother-in-law. Since she moved to Seoul, she has attended a Protestant church and actively participates in the Christian community. For example, she teaches Janggu, which is a Korean traditional musical instrument, to the elderly in her church every Wednesday.

Anmi's case is an example of how a FCH perceives their position in caring for a family member. Firstly, qualifying as a FCH has a multifaceted shape for Anmi. She obtained a care helper licence before her mother-in-law became frail, which meant her qualification was not the result of her mother-in-law's illness. However, her caregiving as a FCH is understood as ‘a proper thing to do’ (Finch 1989) in the sense of the first daughter-in-law’s obligation in a traditional family in Korea.

She expected that qualifying as a care helper could provide opportunities to earn money and perform voluntary activities for the elderly after their retirement. She had information about an LTC programme which allowed family carers to engage in the programme, provided they were qualified and obtained a care helper licence, like other care helpers. Moreover, she tried to put her mother-in-law in an LTC facility at first, when the motherin-law was entitled to Grade 1 or 2 assistance when the recipients could choose to receive LTC facility services.

She could not, however, avoid caring for her mother-in-law at home due to traditional care norms in South Korea and the rigorous grading standard for entitlement to LTC facility services. Although her mother-in-law did not have good relations with Anmi, her first daughter-in-law, she had no option but to stay with Anmi at her first son's house. Neither 
the frail elderly person nor Anmi's husband and family wanted to put their mother in a care facility. Instead, they wanted Anmi to care for their mother under the first daughter-in-law's obligation in the sense of Korean traditional care culture. Anmi's sisters-in-law paid small amounts of money as compensation for her caregiving. The standards for entitlement to LTC services were so strict that the mother-in-law was entitled only to Grade 3 assistance, which did not cover for anything more than in-home care services. These two factorsfamily expectations based on traditional care norms and the LTCI's strict standards for receiving facility care services-forced Anmi to care for her mother-in-law herself, despite her hatred towards her.

There has indeed been a long history of the strained relationship between Anmi and her mother-in-law. Anmi has, since her marriage in her twenties, continuously suffered from her mother-in-law's coldness towards her, which led to Anmi resenting her in turn. However, Anmi repeatedly expressed her feelings regarding her mother-in-law through 'a feeling of guilt' instead of pure hatred. Anmi’s resentment towards and a bad relationship with her mother-in-law cannot generate this feeling of guilt. Rather, there is a power relationship between them, and in this sense, Anmi is in a subordinate position regarding decisions on family issues. What is even worse, the person with the upper hand needs her care in every part of daily life. Anmi's caregiving thus necessarily intertwines with her daily life, her career and even her feelings. Despite her demanding circumstances, she took responsibility for her mother-in-law's care to fulfil her husband's wishes and respect the care norms based on traditional Korean care culture, which places a value on the respect due to old people. These two points (the expectations of the husband and of society) cause Anmi to experience complicated feelings. Anmi generally agrees with the traditional values and tries to meet the expectations, but the bad relationship with her mother-in-law and weak status in the labour market hinder her success, leading to her ambivalent feelings being expressed in the phrase 'the feeling of guilt'.

\section{Type A: Traditionalists}

Experiences of the FCHs who care for their parents differ from FCHs who care for their spouses. Spousal care is more likely to involve emotional bonding and commitment to the person in need of care as a partner in life. In addition to emotional bonding, most spouses who care for the elderly in need of LTC are also relatively old compared to the age of the adult children, and their spouses are already out of the labour market. 
However, adult children's care experiences can be understood in a different context as they have a different status in the labour market compared to spouse carers. Although the male breadwinner model had been dominant in Korea over the past decades, the standard lifecourse of both men and women has started to change since the 1990s because of shifts in the labour market: women's economic participation has increased, and the male breadwinner model is no longer applicable. Present mid- to old-age women, who were born between the mid-1950s and the early 1960s, have experienced dramatic changes in their life-courses and care arrangements.

They have followed changing trajectories of work and care arrangements. They not only manage domestic and care works at home but also are secondary earners participating in the paid labour market to support the household. However, it is hard to say that these changes result in women having an equal social status compared to men, since they ordinarily have to accept career gaps due to child care, and they also have a lower average education level because of the limited opportunity to obtain an education in their teenage years. This career gap and low education levels mean middle-aged women find it hard to get a decent job.

They also mentioned such difficulties in managing both care and work. Most interviewees in this study experienced entering into and retreating from their job in the paid labour market before they started to be a FCH under the LTCI. Bokyung (aged 55, daughter) and Euna (aged 44, daughter-in-law) explained their difficulties in the labour market as follows:

"Euna: as you know, I have three children so I should care for them (Interviewer: I see), daughters. Since my children were young, I couldn't work consistently, rather a little bit, well (interviewer: for a short time, like for a week?) not that, cause my father-in-law helped me. (interviewer: sort of a part-time?) Yes, Yes

(interviewer: From 4 pm to 10 pm, like, like a part-time job? Three times per week, for example?) I could work there for a month." ${ }^{42}$ (Euna, aged 44, daughter-in-law)

\footnotetext{
${ }^{42}$ 그애가 3 명있잖아요.(네)..그러니깐, 자녀양육이죠 (아)딸, 애들이 어렸기 때문에, 이렇게 꾸준히 할 수 있는 일 말구요. 약간약간 뭐 (부업으로 약간, 일주일 이렇게) 그렇게는 말구요, 그래도 아버님이 도와줬기 때문에.. (아니면 파트 같은 걸 하신 거 예요?) 네네 ( 4 시부터 10 시까지뭐요런,요런일같은걸하신거예요?일주일에 3 번 뭐 이렇게. ) 그래도 한 달 정도는 다닐 수 있는 회사 였는데, 네..(176-180)
} 
This section has shown experiences of FCHs by analysing Anmi's case, which is representative of Type A: Traditionalists. The LTCI programme was introduced in Korea in 2008, but traditional care culture norms remain and enforce caregivers, who are mainly first daughters-in-law. Besides, a carer's pre-history with the elderly and family dynamics has impacts on the attitudes and motivations on becoming a FCH. Previous studies on family caregivers mainly focused on carers' experiences on motivations, relationships and care burdens after care situations happened. However, this study focused on cares' life history including their family backgrounds, childhood, education, history with the elderly and other family members, and their working career before the elderly getting frail. This approach enables to capture the whole context about caregiving as a FCH. In the next section 5.3, I will move on to the experience of type B: Negotiators.

\subsection{Type B: The Negotiators}

This section investigates the experiences of another type of FCH by analysing Sunjoo's story. The type of the negotiators comprises four of eighteen interviewees in total, with two daughters and two daughters-in-law. What they have in common is that they care for their parents or parents-in-law based on a feeling of affection for the elderly. With this affection in mind, the interviewees consider their caregiving not just as a burden but also as an opportunity to improve relationships with the elderly as well as their self-esteem and pride as a caregiver. Unlike other types, the negotiators try to share care responsibility with other family members in various ways. In this sense, I also explore how family care helpers (FCHs) are coping with the introduction and changes in the long-term care insurance (LTCI) programme.

Sunjoo is a representative of the negotiators and has distinct characteristics. She has a deep affection for her mother caused by her complicated family history. She cared for her mother long before the introduction of the LTCI, so her case shows a trajectory of recognitions of FCHs according to changes in recognition hours for payments for in-home care. Also, her caregiving is compensated as paid work although it has different characteristics from employment relationships in the paid labour market. Lastly, she was born in the mid-1950s and experienced both rapid economic growth and recession within her and her husband's working ages. These extreme changes affect individual life-courses and their care circumstances. 
This section consists of four sub-sections. Section 5.3.1 explains the interview context describes the context of the interview and how I made contact with Sunjoo. Section 5.3.2 provides a self-presentation of the interviewee. In Section 5.3.3, I identify several key themes to understand Sunjoo's experiences as a FCH. I reconstruct Sunjoo's life story according to the patterns I found in the interview with her. Section 5.3.4 summaries the case of Sunjoo and the type represented by Sunjoo.

\subsubsection{Interview context}

I interviewed Sunjoo in August 2015. This interview was the twelfth that I conducted during my fieldwork. At that time, I had interviewed only one carer who was the daughter of the elderly person they cared for. Thus, I was looking for other daughter cases as I assumed that daughters would act in different contexts to those of daughters-in-law.

I was introduced to Sunjoo by a care helper's association. The association had recommended me to access a care centre that provides in-home care services for recipients of the LTCI. I asked the chief of that care centre to contact interviewees in various family relations, and the director introduced me to Sunjoo. I did not have much information about Sunjoo until I met her. The only information that I had was her relationship with the recipient, i.e. that they were a daughter and a mother. Given that this was only my second such case, I had no idea about the specific patterns of daughter cases but only general and preliminary assumptions about them.

I met her at a café near the convenience store her son ran. My first impression of her was that she was relatively less reluctant to talk about her experience compared to other interviewees. After some questions and answers regarding brief demographic information and ice breaking, I asked her the main narrative questions and she began to talk about her mother's accident at home that led to her needing long-term care.

I found her case interesting in that Sunjoo had a complicated family history that affected her care attitude towards her mother. Another point which makes Sunjoo's case attractive from a researcher's point of view is that she had been caring for her mother for almost 20 years but her mother lives separately from Sunjoo’s family. This is not common in Korea. Although the three-generation family form has decreased, adult children generally still let their frail elderly relatives move into their home when they cannot live independently anymore. Sunjoo’s mother could not live independently - indeed, she could not even walk 
without assistance - but as her mother wanted to live separately Sunjoo respected her mother's preference.

\subsubsection{Self-presentation}

The interview was relatively long though, the interviewee's initial narration was a short one (approximately 5 minutes). The initial self-presentation began with a very brief description of her mother's hip fracture and operation 20 years ago, then a failure to recover. This description was the response to my opening question on becoming a family care helper and the illness of the elderly she cared for. However, right after the description of her mother's health conditions, the interviewee changed the topic to conflicts with her sibling, the more than 20 years of her caregiving, her family's economic difficulties after the Asian financial crisis in 1997, and the changes in payments for the family care helpers. I did not realise this at the moment of the interview, but when I look at it in retrospective, she tried to talk about all of the key events regarding her care work as a FCH except one thing, the absence of mom in her childhood.

After a short description in the first phase of the interview, she gave me detailed information on how she decided to become a FCH. Because she changed the topic to conflicts among siblings after that, I wanted to know more about the constellations in her family. Specifically, I held in mind that elderly care by daughters was not typical in Korea when there was the oldest son in the family. When we talked about her father's death in his thirties, she started to tell me a long story about her childhood and family by saying "because you use this as data, I will say honestly." ${ }^{43}$ When I heard that expression (at 00:18:00 minutes of the record), I expected that she might talk about something important but personal. One can interpret her phrase as a warning that the following story plays a pivotal role in understanding her family constellation and caregiving for her mother but is very personal and or contains sensitive information like, for example, topics that are not socially desirable to talk about. It can also be understood as a reassurance that the interviewer is bound to a principle of confidentiality in this setting. After saying this very phrase, she started to talk about her mother's absence in the siblings' childhoods and Sunjoo's sympathy for her mother and the bad luck she has suffered in her life. Sunjoo's narration was unexpected for me, so I decided to discard my preliminary topic guide to

${ }^{43}$ 선생님이 이게 이제 자료로 쓰신 다니깐, 솔직하게 말씀을 드릴게요 (201-202). 
instead follow her lead. After providing me with such a context, she led the interview towards the thematic field "caring as a reconciliation of the relationship with my mother by understanding her and negotiating with the other siblings".

Over the course of the interview, this thematic field appeared repeatedly. One can identify two different topics in the thematic field regarding family relationships. One is the relationship between Sunjoo and the mother, the other is that between Sunjoo and her siblings. Firstly, Sunjoo explained many episodes from one topic to the next with many relevant narrations. For example, she remembered the day of her mother's accident very vividly and described it in great detail what she saw and how she felt. It is also notable that she changed tenses, sometimes slipping into present tense as if we were on site, for example, she described that the floor 'is' soaked with sweat from her mother when she 'visited' her apartment. This way of speaking gave me the impression that she was very shocked by her mother's accident. Apart from describing the day of the accident, she showed her affection for her mother by explaining why she decided to care for her mother at home instead of sending her to a nursing home, even though she had been accessed as Grade 2 and therefore been eligible to choose facility care services. She told me several reasons, including her mother's fear of the nursing home, and Sunjoo's explicit will to care for her. In the course of the narration, she mentioned she did not want 'yet' to send the mother to the LTCI facility. With her using the adverb 'yet', one could formulate several hypotheses. For example, she could afford to care for her mother for now, but she expected it to not last forever, rather sometimes she would send the mother the nursing home. Or, one could set up a hypothesis that she and her mother have negative images of the nursing homes in Korea so they might wait until the overall conditions in the facilities improve. Or, one could assume that she has been frustrated with caring for the mother as she has cared more than 20 years alone without any help of her siblings.

Another main topic of her narration was conflicts between her and her siblings. She repeatedly criticised the siblings' indifference towards the mother's condition. She strongly and repeatedly insisted that although her mother had left her children, she has had a demanding and hard life as a woman and became frail due to ill fate, so they have to understand and help her. This can be interpreted as a way to convey her care burden caused by long-period family care without any help from the other siblings. However, one can also question why a child shows affection and feels responsible for a mother who did not take 
care of them at all, and what makes Sunjoo keep caring for the mother and expect her sibling to do the same? I set up the hypothesis that caring for her mother less the result of affection but more an attempt and a continued process to develop affection for her mother. In this sense, I assumed her desperate attempt to continuously negotiate to care for the mother is not just because of the care burdens regarding time and resources, but because of family values, she wants to achieve.

Lastly, she was the only interviewee who linked the payments for family caregiving with cost-sharing between state and family at an abstract level. When she mentioned her doubts on the payment for family caregivers at the implementation of the LTCI in 2008, I found her sentence as a rhetorical sentence to express her wonder. However, she also spoke about the experience of giving birth to her third child in 1984 when the national health insurance (NHS) did not cover childbirth costs for a third child due to the population control policy in the 1980s in Korea. Taking this into consideration, I assume her experience with other social insurances made her suspicious of policies in the context of the Korean welfare state, including the LTCI scheme.

\subsubsection{Reconstruction of Sunjoo's life history}

Sunjoo was born in 1956 in Seoul when Korea was in an economically weak phase in the aftermath of the Korean War, and she has lived there all her life. Her youth was spent under the first dictatorship that took power in a military coup in 1961 and lasted until 1979.

At the time of the interview, she lived with her husband and three children (two daughters and one son). She has now cared for her mother, who suffers from physical and cognitive impairments, for nearly twenty years.

\section{Family background and childhood}

The earliest moment she talked about in her childhood was when she was eight years old. At that time, her father had died, and the incident was one of the most decisive events in her life. She did not go into detail about her father's death but explained the fundamental changes it caused in her family. Sunjoo's mother was still young when her husband died, in her early thirties. Later, she decided to remarry and leave her children. From her present perspective, Sunjoo says she understands her mother's situation. In the 1960s, women's 
economic participation was meagre compared to the present, and at that point, her mother had four young children to support on her own.

Since Sunjoo’s mother had left her children, the children had to find new places to live. They were consequently brought up by their late father's relatives. Sunjoo is the second child and has one older brother and two younger sisters. She lived with one of her father's elder sisters (the aunt of the children), and the others lived with their father's mother (the grandmother of the children). When asked for more information about why she lived separately from her siblings, she did not provide any detail on that; she assumed that it had happened because the aunt was married but had no children, and she had always liked Sunjoo. After Sunjoo’s mother had left, the aunt and the grandmother respectively became the children's caregiver who supported them both financially and emotionally. There was no contact between mother and children until Sunjoo’s own marriage.

\section{Living together with an aunt instead of mother and siblings}

Sunjoo lived together with her aunt and uncle (the husband of the aunt) who covered her education expenses. Since the aunt's family had a factory and ran their own business, they could afford to send Sunjoo to an academic high school before entering university. Unfortunately, however, she had to give up going to university because of a sudden decline in the family's economic situation. The factory the aunt's family ran was burned down following an accident and as a result, they could no longer afford tuition fees for Sunjoo's university. She remembers this in the following terms:

"The factory was burned, (interviewer: ah) and they had a difficult time. (interviewer: I see) Well, my aunt wanted to educate me more, but I told her by myself (interviewer: ah okay) 'I would like to give it up'. Then, my aunt responded that I would probably regret it, so I said 'I won't regret it'. However, I do regret now that I could not study. Since the strap of my bag is too short ${ }^{44}$ so that I have no choice in job and career" 45

This statement is interesting to me because her education level is not low compared to most females her age. She was born in the mid-1950s and at that time less than $50 \%$ of the girls

\footnotetext{
${ }^{44}$ This is a Korean fixed expression, which refers to the status of people who are less educated.

45공장에 막 불이나고 막 이래가지고 (아) 어려움이 있으셨어요. (아 그러시구나) 그래가지고 우리 고모가 더 가르치고 싶었는 데 내가 알아서 (아,네) 안다니겠습니다 그랬어요. 그랬더니 '후회한다' 그런거야 ‘후회 안할게요' 그래서. 그런 데

지금 후회합니다. 공부 못한게, 가방끈이 짧기 때문에, 제가 어디가서 이렇게 문을 두드리고 할 수가 없잖아요. (1154-1159)
} 
went to high school (Ministry of Education 1975). However, she felt she less educated, and the deficit in her education stemmed from her family background.

"As I mentioned in relation to my situation before, I lived in my aunt's house (interviewer: okay). I know she did her best for me, I don't know why, but I felt a little bit wounded. So (interview: because you were young?) yes, yes, for example, about tuition fees" 46

Furthermore, her statement indicates that she was not forced to give up going to university, but rather felt she could not impose on the aunt given the family's difficult situation. Also worth noting is that she blames her difficulty in finding a satisfying job in later life solely on not getting her university degree while also other factors, like career interruptions caused by her marriage and childcare and elder care for the mother, can play a part in this.

After graduating high school at 19 in 1975, Sunjoo found work in an office in Seoul where she did paperwork. It is clear that she was well-educated enough to get a relatively good job compared to many females of her generation. Once she managed to secure a job, she became economically independent and even paid her younger sister's tuition fees. She talked about her devotion to her two younger sisters and how she and her sisters lived in poverty at that time. By bringing up this topic, she shows her regrets regarding her younger sisters. When Sunjoo was financially supporting them, they respected their older sister and agreed with her decisions on caring for her mother. However, after the sisters grew up enough to live independently as well, they allegedly did not anymore. Rather, there was a long period of conflict between them, especially in regards to caring for their mother.

Sunjoo married one of her work colleagues in 1979 when she was 23 years old. She met her mother again after she married. She had sometimes heard news about her mother from her aunt, but she says she did not ask further as she considered it impolite. When Sunjoo met her mother again, she found out that her mother's second marriage had broken down; she was divorced and lived on her own in a small apartment nearby.

Sunjoo does not offer many details about her family life with her husband and children before her mother has had a sudden accident. There are many gaps in her life story regarding this period. We do know that she stayed at home as a housewife after she married. 
She has three children and her husband worked a white-collar job and held a permanent position in his workplace. Her household was not excessively well-off, but they enjoyed an ordinary life in the 1980s and 1990s. The Korean economy thrived during the 1980s, specifically between 1986 and 1988 when there was a Korean economic boom called the 'three low economic booms' when the cost of petroleum, the value of the dollar, and the international interest rates remained low, factors that once aligned allowed for economic growth (Shin Kyung-Ah 2013; Wang 2013a; Shin and Chang 2003). In this period, Sunjoo's husband was employed at a big company with more than 500 employees. To her own assessment, she had led a basic middle-class lifestyle and had few hardships at that time. This background made it possible for her to care for her mother by herself without the help of her siblings (or the state).

\section{Her mother's accident and the conflict between the siblings}

In 1995, Sunjoo's mother fell and fractured her hip at home. Sunjoo still has vivid memories of the day. Since the mother lived alone, Sunjoo called her every day to check on her but on the day of the accident her mother's line was busy all day long, as she had fallen on the way to answer the telephone. Despite all her efforts to control her body, she could not move or make a sound at all. When her daughter visited her apartment, the floor was soaked with the sweat of the elderly woman. At this time, only Sunjoo had contact with the mother so it was she who found her and called the ambulance service to take her to hospital.

When Sunjoo's mother fractured her hip, she was 67 years of age and thus relatively young: she could, therefore, have recovered if she had followed the rehab guidelines. Sunjoo remembered that the doctor strongly recommended follow-up treatments for her hip operation. However, Sunjoo's mother misunderstood her doctor's advice and lay in bed without moving instead of undertaking an intensive and active rehab. Thus, Sunjoo tried to encourage her mother to live an active lifestyle but it did not work:

\footnotetext{
"So, when we visited mum and said, 'you should work out somehow, mum... move like this', but she was disappointed about it and said 'I am very sick' (yes) 'Since you are not sick, you treat me like that', she thought. (yes) So, more than the feeling of disappointment, I thought 'Okay, then mum maybe feels more comfortable, and she knows better', and I said 'Okay, do whatever you like to do', So, for a substantial period, she had lain back, hadn't she? So the wound closed up. However, what had happened was that, because she had lain back
} 
still there, the metal pins had been thrust up when she tried to move again. So, it hurt a lot. So, she tried to rehabilitate herself, tried to like that, but it didn't work due to the piercing pins." 47

As seen above, Sunjoo recommended their mother to work out but she found it uncomfortable both physically and emotionally. Finally, the lack of rehab worsened the mother's health condition. Sadly, it is not uncommon for old people's condition to worsen after an operation since the rehabilitation is not well linked to ambulant treatment (Chang Jun-Dong et al. 2010: 287; Kho Duk-Hwan et al. 2009). Sunjoo’s mother tried to have surgery to remove the incorrectly settled pins but the doctor hesitated to undertake surgery again since the elderly are particularly vulnerable to the side effect of general anaesthesia, which, in severe cases, can cause death. For this reason, Sunjoo and the mother decided to give up on her having another operation, as they agreed it was better to remain in the present condition rather than to die. As Sunjoo and her mother decided not to have surgery, she failed to recover her mobility and could no longer walk freely. They had to move onto the next stage, which was therefore long-term care.

\section{Caring for my mother: The reason why I take the responsibility}

As mentioned before, according to traditional Korean care culture, the oldest son used to take care responsibilities for the parents. Even though Sunjoo's mother has four children (one son and three daughters), Sunjoo was the only one willing to take responsibility for her care, with the other siblings being disappointed and upset about her having left them at a young age to remarry after the unexpected death of her first husband.

"So, I cared for mum. Although there were many siblings, they were not of the same mind. So, being not of the same mind, maybe, they wanted to help, they couldn't do that in real. But in doing so, now, it's fallen to me. Well, so, I was upset about the siblings, so very upset, yes. I sis, but it has been already for 20 years, I was in my forties at that time, wasn't I? At that time, I was vigorous,

\footnotetext{
${ }^{47}$ 그러니깐 우리는 가서 ‘엄마 운동좀 해야돼, 엄마 이렇게 움직여 봐’ 뭐 이렇게 이야기를 하며는, 그걸 섭섭하게 생각을 하시는 거예요 ‘내가 이렇 게 대단히 많이 아픈데' (네) 니가 아프지 않으니깐, 이렇게 나한테 막하는 구나. 이런 생각을 하시는 것 같아요(네) 그러니깐 섭섭한 것 보다도, 아, 그러면 엄마가 편안하고 당신이 더 잘 알겠지 생각을 하고 ‘응, 엄마 편한대로 하세요' 인제 그랬어요. 그래가지고서는, 상당히 오랜 동안 누워계셨을 것 아니예요? 그랬는 데, 그게 인제 아물었는데, 어떻게 되었냐 하며는, 그낭 그대로 얌전하세 계셨으니깐, 이거 움직일래니깐, 이 쇠가 딿고 나오는 거예요. 그래가지고 고생을 많이 했어요. 그랬는데, 고거를 이제 재활도 좀 하고, 이렇게 좀 할라고 그랬는데, 그게 이제 쇠가 이렇게 살을 뚫고 나오니깐, 그게 안되는 거야(69-75).
} 
so 'Okay, without you (interviewer: yes), anyway, I can just take care of my mum', and in that time I could afford to care for mum." 48

I did not hear about any details or episodes regarding how Sunjoo’s siblings felt negatively and responded to their mother's accident. From Sunjoo's account, it is evident that the other siblings did not visit the mother anymore; only the youngest sent a little money, about 50,000 won monthly.

Sunjoo decided to take care of her mother for several reasons. Mainly, taking an objective point of view, Sunjoo sympathises with her mother, as a woman who suffered a miserable life and could not help leaving her young children. It is obvious to her that it was hard for a young widow to earn a living and to care for four young children at the same time. After all, she was only in her thirties when her first husband had died. When asked about her motivations for caring, Sunjoo expressed her thoughts and feelings as follows:

"I found mum so pitiful and pathetic; these feelings come over me above all (interviewer: okay). And she was ill. (interviewer: ah okay) As a woman who lives a miserable life, she is pitiful (interviewer: all right) I feel so pitiful. So 'anyway it was not hard for me to care for mum, for only one person' (interviewer: okay) though I hated her, I missed her too. (interviewer: all right). Well, that's why." 49

Apart from Sunjoo's attempt to empathise with her mother's situation as a woman, her account above shows other reasons for her decision to care for her mother. She wanted to protect her mother, who is ill and frail. In the latter part of the interview, she talked about a family quarrel regarding caring for their mother, and she persuaded her sisters to protect their frail old mother. Whereas these rather ethical motivations originated from her general and humane perspectives, her third motivation is based in the conflicting feelings of hatred, love and pity towards her mother, which are based in childhood and her separation from her mother. Since she had less contact with her mother in her early life, caring for the frail old lady could be a way to make up for this and change their relationship for the better.

\footnotetext{
48 그래서 인제 엄마를 모셨는데, 형제가 여럿이 있는 데, 다 똑같은 마음은 아닌 거더라구요. 똑같은 마음이 아니니깐, 마음이야 인제 다 도와주고는 싶겠지마는, 현실적으로는 이제 그럴 수가 없었겠지요. 근데 이렇게 하다 보니깐, 이제, 내, 내몫이 된거야. 그런 데 이제, 많이 속상하고 형제들한테, 많이 속상하고 막 그랬었는 데, 지금 20 년 되었으니깐, 그때만 해도 내가 40 대 뭐 이랬을 것 아니예요? 그때만 해도 내가 혈기에 '그래 니네가 안하며는(응) 내가 뭐 엄마 한 분 정도는 내가 모실 수 있어’그러고 그때 상황이 뭐 그래도 내가 모실 수 있는 여건이 되었어요 (81-85)

49 엄마가너무 불쌍하고 너무 안됐고 그런 마음이 우선은 들었어요(네) 그리고 아팠잖아 그렇게 (아,네) 그리고 또 이렇게 보며는 측은한 감. 그 여자 일생으로서 봤을 때 (맞아요. 맞아요) 너무 안된 마음이 들었어. 그래서 '아 내가 뭐 엄마, 엄마 한 분 정도는 내가 할 수 있어' (아,네) 또 미움도 있지만 그리움도 있거든요? (맞아요) 네, 그래서 (322-325)
} 
Lastly, she was also able to afford to care for her mother from a financial perspective since her husband had a regular white-collar job and their economic situation at the time was relatively secure.

However, she did not know how long she would have to care for her frail mother. Even if she anticipated decades-long care for her, she was not aware of what exactly such longterm care responsibilities would mean to her life. She expressed her frustration and remorse regarding her lost golden forties in the late part of the interview. Worse, her husband had lost his job after the Asian financial crisis of 1997 and the family was in a precarious financial situation. They had never thought he would lose his job, and there was no means for them to make a living.

"However, in the caregiving, [I] have become poorer due to the IMF ${ }^{50}$. So how it would happen (interviewer: yes), well, to educate the children, even three (interviewer: I see) three [children] to the universities at once, three should go. So, it has come hard." 51

Sunjoo's husband received an early retirement package as a lump sum, which he invested in starting up a new business, but also had to be spent on ordinary living expenses, and the university tuition fees of the three children. However, the company her husband founded became bankrupt after a short period, and he had to settle for a low-paid job as a security guard. Sunjoo also had to work part-time from time to time. She reflected that at times she 'often felt like she wanted to die'. Her extreme choice of words indicates what a heavy effect the unexpected events in the life course of her and her husband had on her. Korea had experienced rapid economic growth before the Asian financial crisis in 1997 and lifetime employment had traditionally been common in Korea. Therefore, such a sudden change caused by external socio-economic conditions had a huge effect on many individual life-courses (Hahm Inhee 2012; Yang Sungeun 2012).

The economic crisis in the late 1990s in Korea resulted in a shortage of jobs, which resulted in massive layoffs. Sunjoo's husband was dismissed from his regular white-collar job in his forties. Unexpected layoffs like this can have a lasting effect on the next stages in a

\footnotetext{
${ }^{50}$ Usually, South Koreans call the Asian financial crisis of 1997/98 the IMF (International Monetary Fund) since the Korean government applied to the IMF for a bailout.

${ }^{51}$ 근데 하다 보니깐 뭐 IMF 터져서 생활도 어려워졌고, 뭐 인제 이렇게 될 거 아냐(네) 애들 뭐 교육도 셋이나 (그초) 한꺼번에 대학을 셋, 셋 갈 정도니깐, 그러니깐 지금은 이제 어려워지는거야 (86-89)
} 
person's life course. In fact, her husband was never able to get a well-paid permanent job again that secures an entitlement to a social pension, unemployment benefits, and compensation for occupational accidents.

Sunjoo cared for her mother informally for more than ten years until she heard about the introduction of the LTCI, within which family carers could be enrolled. At first, she doubted that the state would compensate her for caring for a family member. Her doubt originated in negative experiences she had relating to other social insurance services:

"I appreciated it, because, as I know the national health insurance (NHI) was introduced in $1978^{52}$ (interviewer: yes), for employees worked in a company which employ more than 500 workers, (interviewer: yes) we became entitled (interviewer: yes) but I was limited covered since I had given rise to three, three children. (interviewer: yes) in that time, birth control policies were imposed. (interviewer: it was entirely different from now) That's why I told my son that the state did not confer a benefit on you. I said." 53

As South Korea's estimated population exceeded 40 million people in 1983, the state implemented birth control policies that discouraged people from having more than two children. Besides a campaign promoting birth control, the state also implemented active measures such as disadvantages in income taxes and the contribution rate for the NHI, as well as a limitation on family allowance and education provision for the third child of civil servants (Wang Hye-Suk 2013b). For Sunjoo, the cost of the delivery of her third child was not covered by the NHI due to the birth control policy. Through this experience, Sunjoo realised that the state could not always support individual needs. Instead, the state puts the collective good of society before the individual's needs and demands.

\footnotetext{
52 The national health insurance was implemented in July 1977, which corresponds approximately with Sunjoo's recollection. At first, the NHI only covered for employees in large-sized companies who hire more than 500 employees, but entitlement to the NHI has expanded to people, including employees in smallsized companies as well as civil servants and the self-employed. Kim Young-Bum (2002) pointed out that the state implemented the NHI for employees in the big business to ensure a reliable supply of labour force in the heavy and chemical industries, which means social policies went in tandem with the economic policies of the time.

53 근데 내가 이제 고마운게 뭐냐며는, 내가 알기로는 78 년도에 의료보험제도가 그 때 처음 생긴 것 같아요 (맞아요) 500 인 이상 (맞아요) 우리가 그때 혜택을 했던 거거든 (네) 근데 나는 애를 셋을 셋을 낳았다고 혜택을 못받았어요. (아, 네) 그때 산아제한 할 때야 우리는. (지금이랑은 완전히 반대네요) 그래서 내가 그랬어요. 우리 아들한테, 나라에서 너는 혜택을 주는 게 없다 내가 (721-725)
} 
After Sunjoo became aware of the LTCI programme and the possibilities it provided, she decided to become a qualified FCH for her mother. One of her friends encouraged her to take courses so that she could obtain a care helper's licence, and she did so. After completing the course, she became a FCH and signed a 'working contract' with the care centre she was introduced to by the friend.

\section{Daily life incapable of ADL and IADL}

In addition to Sunjoo's obtaining a care helper licence, her mother also needed to be assessed and graded for using LTC services.

Since Sunjoo's mother lives alone, Sunjoo's family visit and provide care every day. Although her formal care recognition is one and a half hours (7:00-8:30 am), she usually stays all morning. Due to the mother's physical impediments, her daughter does all the household chores such as cooking, clothing, cleaning, and the laundry, as well as bathing her once a week with assistance from another care helper. Even though Sunjoo's mother cannot perform the activities of daily life (ADL) and the instrumental activities of daily life (IADL) by herself, she wants to maintain her living environment and stay at home alone as she did before her accident. To respect her mother's wishes, Sunjoo visits her every day rather than having her mother move in with her or placing her in a nursing home. Sunjoo’s children also help her; her son sees her off and picks her up every day, and sometimes he takes the grandmother for a ride.

\footnotetext{
"Yes, at first, we lived together six in total (interviewer: ah) my-side (mother?). Yes, so now, well, something happened (interview: the mother lives separately) the mother was out, (interviewer: ah) well, it might be considered that we live together (interviewer: right). Because I take my mother into my home, and she stays for 2-3 days and again takes her back (interviewer: umm), so we are apart, [she] wants to be alone." 54
}

While caring for her mother for over twenty years, Sunjoo has faced many care burdens. When asked about these caring burdens, she mentioned first and foremost the lack of flexibility in time and mobility. She expressed this as having 'broken the rhythm of life'. When her mother had the accident in the bathroom, Sunjoo's children were teenagers so

\footnotetext{
54 네, 원래는 6 식구가 살았죠. (아) 저희 친정 (어머니까지?) 네네, 그래서 지금 이제 사정에 의해서(어머니가 가시고) 어머니가 가셨는데, (아) 뭐 같이 산다고 볼 수도 있는 거예요(그초) 왜그러냐며는 저무도록 모시고 뭐 왔다가 또 뭐 2-3 일 있다가 또 모시고 가고(음) 그니깐 당신이 이렇게 떨어져 있는, 혼자 계시는 거를 원하시니깐 (54-56)
} 
she had a double care burden with having to look after her children as well as after the needs of her mother. There is a lot of research on sandwich carers who face double demands for two different kinds of care work (Choi Won-Seok and Lee Jin-Sook 2014; Marrs 2011).

Another burden she mentioned was difficulties in communication with the cognitively impaired mother. Although she tries to keep her mother's illness in mind, she sometimes cannot bear the elderly woman's stubborn assertions based on her delusions, which has led to quarrels sometimes.

Apart from the emotional burden in regard to her mother, Sunjoo also suffers under the strained relationship with her siblings. She is the second child and has one older brother and two younger sisters. However, the brother and the youngest sister do not care for their mother or have any contact with her, and the third sibling only sends Sunjoo money to support their mother. As mentioned above, this indifference stems from family history, particularly the mother's remarriage and her children's negative feelings about their separation. Unlike the other siblings, Sunjoo tried to understand her mother's position so that she could accept and feel sympathy for her mother as a woman like her. Furthermore, she feels that her other siblings also ought to take care of their mother since they 'have grown up enough to understand her and she is already old and frail'. Sunjoo is disappointed with her sisters' attitude. This also relates to the fact that she feels her sisters are unthankful as in that she supported their education when they were younger but they, in turn, are unwilling to support her with the care burden now.

As well as the general aspects of the carer's difficulties, Sunjoo has faced other problems due to the prolonged care work, which made her both physically and mentally tired. As the years go by, her physical and mental burdens have increased, and according to herself she often feels regret that she 'spent her golden forties caring for her mother' and has considered transferring her mother to a care facility more than once.

\section{The LTCI is better than ten sons}

Sunjoo evaluates the FCH programme in the LTCI as positive in that the programme. To express her satisfaction with the programme, she used a traditional Korean saying: "the LTCI programme is better than ten sons”. However, the proverb presupposes that obligations of care for the elderly originally belong to their children. This attitude towards elderly care originated in Korean traditional care culture in the context of patriarchal 
Confucianism. Sunjoo is so convinced by this attitude that she asserts children's obligation and filial piety towards their parents should be required by law:

"Sometimes I said that it is necessary to legislate against children who do not care for their parents and to force them by law to pay. (Interviewer: you mean undutiful children) Yes, the undutiful children, yes, yes. (Interviewer: be fined as a penalty?), such such, we need to legislate such, such a law, I said." 55

This remark suggests the interpretation, that she feels powerless in face of the indifference of her siblings and their continued refusal to help her with their mother even after all these years. Therefore, she imagines a scenario where her siblings would be obligated to care for their mother and could not simply leave her with an increasingly burdensome responsibility.

Besides, her attitude towards care work implies that she understands care not only as labour and expense but also as having an emotional component. High-quality care services offered by non-familial care professionals lack a pivotal element of care for the elderly. Moreover, her mother has always resisted entering a nursing home claiming she is afraid of something bad happening to her there.

Another of Sunjoo's care attitudes in relation to the LTCI is that public care provisions are not only for the patient (the elderly person) but also for the family member who cares for them. Although Sunjoo is willing to take responsibility for her mother, the responsibility of the family should be shared with the state via financial support or compensation for the care. She explains:

"Not only patients but also we family members have such rights, haven't we? (interviewer: yes) Well, I wish it works for us too. For mum, well, she doesn't have long to live. I think. It is enough for me now, but a little bit more then I could live more like a human being." 56

Sunjoo suggested extending the time covered by compensation for LTC offered by family members. She is compensated for her care service as a FCH for only 90 minutes per day,

\footnotetext{
55그러니깐 어떤 때는 그말 했대니깐'법에서 그 자식들이 돌보지 않으며는 강제를 해가지고 강제로 돈을' (불효자를) 응, 불효자들, 어, 어 (벌금 내라고?) 그런, 그런 법 좀 안만드나?' 내가 그랬대니깐.(1125-1127)

56 네네, 저도 그렇습니다. (오히려) 환자한테도, 그런 권리도 있지만, 우리 가족한테도 (맞아요) 그런 권리가 있지 않습니까? 그, 그런 건 좀 잘 되었으면 좋겠어요. 엄마가 뭐 얼마를, 이제는 사실 날 이제 얼마나 인제 그렇잖아요. 저는 그래요. 이것도

지금 감사하긴 하지만은, 조금 더, 그러면 나도 좀 인간답게 좀 (맞아요) 네네 이렇게 (1034-1036)
} 
adding up to about 400,000 KRW (about 400 USD) monthly. The 90 minutes per day is the maximum recognition rate for FCHs, and the rate is only accepted in limited situations. For example, Sunjoo's mother is a case wherein elderly people are diagnosed with dementia such that only family members care for them.

Sunjoo obtained a care helper licence very early on, almost during the introduction period of the LTCI in 2008, which means she has experienced all of the changes in the regulations and payments for FCHs. In the early days of the LTCI, there were no differences being made in payments for familial and non-familial care helpers.

When the LTCI was introduced in Korea, the family-based traditional care culture was still pervasive, thus the government was mainly concerned with insufficiencies in the care infrastructure rather than fiscal matters like payments for licenced family carers. Moreover, the LTCI regulated funding, licensing and assessment systems as a public provision scheme for the LTC in Korea through the education for care helpers and service providers was mainly in the hands of the actor in the private sector. According to An Mi-Young (2017), this service delivery of the LTC in Korea is a public-funded and private-provision system. For the Korean government, the LTCI scheme was not only a social policy but also an economic policy, which was called a new growth engine industry. However, there were far more FCHs than the government expected: the percentage of FCHs who offered in-home care services to their family members accounted for almost $40 \%$ of the total care helpers who offered in-home care services.

Later, the government limited the recognition hours for FCHs from 4 hours per day to 90 minutes per day after 2009. This did, however, not apply to FCH who did not cohabit with the recipients. Despite the regulation, the number of FCHs has not decreased. Therefore, the government cut back the recognition hours for the FCHs again from 90 minutes per day to 60 minutes per day and 20 days per month in 2010. Unlike the first cutback, this one applied to all FCHs regardless of the cohabitation situation. According to the government's Notification, the cutting back of the payments for FCHs is justified by the fact that responsibility for elderly care primarily lies with their family members.

Nevertheless, the government set exceptions for two cases in which FCH can get more hours recognised. The first case is the FCH being a spouse of the recipient of the LTCI, and the caregiver being over 65 years old. The second case is the recipient of the FCH being 
severely demented so that there is no carer who can care for the patient because of his or her problematic behaviour except for family members. However, even a FCH cares for a recipient confirmed as an exceptional case, they still do not get the same hours recognised like non-familial care helpers. Instead, they also received limited time recognitions (90 minutes per day, 30 days per month) compared to non-familial care helpers (240 minutes per day).

Sunjoo has thus experienced most of the changes in payments for the LTC services offered by FCHs. As explained before, in the beginning, she had been getting 240 minutes per day for her caregiving for her mother recognised, which was the same rate as for non-familial care helpers. Despite the limitation in the recognition hours offered by FCHs in 2009, there was no change in payments for Sunjoo as she lived separately from her mother. Following the amendment of the government Notification on the payments for FCHs in 2011, the recognition hours for in-home care services Sunjoo offered for her mother were drastically cut back from 240 minutes to 60 minutes. The experience of changes in payments for care offered by licenced FCHs in the LTCI programme is one of the key themes of this study, so I usually asked my interviewees a follow-up question after they had finished their main narrative accounts. However, Sunjoo brought up her experiences regarding the unexpected changes in the payments by herself, saying:

"Well, during the period, the Asian financial crisis happened so that my living conditions got worse, well something like that (interviewer: yes). However, I had three children to educate (interviewer: okay). My children were supposed to go to universities at the same time so the situation got worse. At this moment, I heard 'Ah, such a programme would probably be introduced.' So, I obtained a licence as a care helper. After obtaining, I did works, at first, more hours were compensated, more compensated." 57

The main narrative account of an interview is the interviewee's self-organised response to a general question like "please freely talk about what makes you care for your family as a licenced carer in the LTCI”. Sunjoo began talking about her situation, for example, her

\footnotetext{
57근데 하다 보니깐 뭐 IMF 터져서 생활도 어려워졌고, 뭐 인제 이렇게 될 거 아니야(네) 애들 뭐 교육도 셋이나(그차) 한꺼번에 대학을 셋, 셋 갈 정도니깐, 그러니깐 지금은 이제 어려워지는거야 ‘아, 이런 제도가 생긴다’고 그러니깐, 이걸 딴거죠. 따라지고 이렇게 하다 보니깐, 맨 처음에는 시간을 좀 많이, 많이 주셨어요. (85-88)
} 
decisions regarding caring for her mother and conflicts between her and her siblings in mostly general terms; but then she suddenly mentioned the topic time recognition.

\section{Reporting and controlling: Care centre and RFID}

For reporting and compensation for care, a care helper of the LTCI should register with a care centre. When the care helper registers and makes a working contract with a care centre, then the care centre manages care schedule and reports the services the carer offered to the NHIS (national health insurance system). Then, the NHIS pays fees for the services to the care helper via the care centre. Family care helpers follow the same payment process, i.e. they make a working contract with a care centre and attend once a month for a refreshing training. Sunjoo, too, follows this process: she was paid for care via a care centre from the NHIS after signing a working contract. Additionally, a social worker from the care centre visits the mother's home once a month to supervise their living conditions and the FCH's caregiving.

Concerning the service delivery of the LTCI, she mentioned that the system, including making a contract with and supervision by the care centre, was reasonable in that it was more comfortable for helpers and could reduce expenditure:

"I think I am a very honest person (interviewer: I see). I am really (interviewer: rather you do more, all things) yes yes, I, I really honestly care for. Well, I think, you know the top-up device [RFID] (interviewer: yes), I like the device (interviewer: umm umm), I (interviewer: because you don't need to write a report?), yes, like it so much" 58

She mentioned the RFID (Radio-Frequency IDentification) system, which is an electronic recording device that cares helpers tap whenever they start and finish their in-home care services. Both the beginning and the end of caregiving sessions are electronically recorded by touching a chip card, which connects a cell-phone with the card reader (RFID) at her mother's home, and the LTCI can thus check whether care helpers perform their work at a given time and place. She says she doesn't mind the electronic check-in and out system because she is honest.

\footnotetext{
58 나같은사람은 정 말 정직한 사람이거든(네) 나는 굉장히(더했으면 더했지, 집안을 다 그렇게) 나는 네네, 나는 사실 굉장히 정직하게 저거를 하거든 근데 그래 난 그래 지금도 이거 선생님 찍는 거 있잖아(네) 그거 나는 아, 너무 좋아요(음음)

나는(일지를 쓰지 않아도 되고) 네네, 너무 좋아요(877-879)
} 
She told me, she did not mind reporting her caregiving to the care centre and the NHIS, but she insisted at the same time that care recognition hours should be longer and flexible for ‘faithful’ FCHs:

"When I am busy, or I have less to do as the patient is well, then it would be nice to finish earlier (interviewer: I see). For that, we first need faith."59

\section{Use of the payments}

One of the most important reasons to become a FCH is to be paid for family care in the formal scheme, but little has been known about the use of payments. Rather, there have been a number of studies on the motivations and burdens of family care. However, investigating on use of the payments enables to understand that for whom and what the payment is compensated. Technically, the payments for care is paid for a service provider instead of a service user, i.e. the NHIS pays the fee to a care centre according to a service report, and then the care centre pays to the family care helper according to an hourly wage they contract at the beginning. However, in most cases, family members share their economic resources, or the adult children support their old parent economically, so, it is not clear who really gets the money in practice. Moreover, most of present elderly are less likely to have their own pension benefit since the NPS (national pension system) had been implemented in 1988, meaning that the first old-age pension benefit was paid in 2007.

Due to her mother's cognitive impairments, Sunjoo's care recognition hours as a FCH count as 90 minutes every day, including an additional 30 minutes per day and ten days per month more than other family carers whose recognition hours are counted as 60 minutes per day, and twenty days per month.

Her income related to her mother comprises a basic pension benefit $(200,000 \mathrm{KRW}$, or 200USD), payment for caregiving as a FCH (400,000 KRW, or 400 USD), and support for her third child (50,000 KRW, or 50 USD). Her costs of living include food, medicine, public utility bills, and spending money. Sunjoo manages both the income and the expenses and gives her mother only some spending money directly. She mentioned that the boundaries of ownership of the payments were vague, i.e. the money could be shared and also applied to Sunjoo’s household. Regarding social security for the elderly, Sunjoo’s

\footnotetext{
59 내가 뭐 급한 일이 있대든지, 오늘 뭐 환자가 뭐 얌전하게 뭐 잘 저기하고 계셨으니깐 뭐 볼 일이 없대든지, 그러면 일찍 찍고 저기하고 그럼 좋잖아요(네) 서로가 첫째는 믿음이 있어야돼(891-892).
} 
mother has no public pension under the national public system (NPS) based on contributions- Instead, she receives tax-based benefits called the basic pension, which amount to 200,000 KRW (or 200 USD) a month. Even though the mother was aware of the payment for the LTC, the elderly woman did not ask her for it.

\section{Sunjoo's household}

While she took responsibility for her mother, Sunjoo did not have enough money to prepare for her retirement years. So far, neither she nor her husband meets the requirements for the pension benefit; she has contributed to the NPS for only three years, and her husband is a low-paid worker now who is not entitled to the pension benefit and the unemployment benefit. Moreover, they have burdened themselves not only with the care of Sunjoo's elderly mother but also with supporting their children, who have not yet married and cannot afford an independent life. Sunjoo mentioned that she always feels sorry for the children, in particular for her son, who has a student loan and was attempting desperately to get a job. Finally, she decided to open a small convenience store for him.

\subsubsection{Structural features summary}

Sunjoo was born in 1956 in Seoul, the capital city of Korea. Her father worked in an office and her mother was a housewife (born in 1928). Sunjoo has one older brother (born in 1953) and two sisters (born in 1960 and 1963 respectively). In 1964, Sunjoo's father died from the disease when Sunjoo was at 8 years old. Her mother had left her four young children to remarry after the unexpected death of the father. Therefore, the children were brought up by relatives from the father's side; Sunjoo lived with one of the aunts (the late father's sisters) and the others with their grandmother (the late father's mother). Sunjoo's aunt was married, had no children on her own and ran a factory as a family business. Sunjoo lived in a caring and well-off atmosphere and entered a high school in 1972. A year later, the aunt's family became in bankrupt as the factory they ran was burnt down in a big fire. Sunjoo gave up going to university. Instead, she found an office job in 1975, aged 19. She saved her salary and supported her two little sisters, paying their high school tuition fees. She met her husband (born in 1950), who is from a city in the southern part of Korea, at her workplace. They married in 1979 when Sunjoo and her husband were 23 and 29 respectively. 
Meanwhile, Sunjoo's mother did not maintain her second marriage but broke up again in the late 1970s. Sunjoo and her mother met again at her wedding for the first time since the mother had left her children in 1964. After her mother broke up her second marriage, she delivered food on the street for a living and had lived alone in a tiny apartment. From 1979 on, Sunjoo and her mother grew closer again. Sunjoo gave birth to two daughters in 1980 and 1982 respectively. In the early 1980s (not exactly the year but before the birth of Sunjoo's third child), Sunjoo's mother had a stroke and stopped her work of the food delivery service. In 1984, Sunjoo gave birth to a son, she was 28 by then. The national health insurance (NHI) did not cover childbirth cost for the third child due to strict population control policies in the 1980s. Therefore, her family had to pay the medical bills for the birth of the third child by themselves.

Sunjoo's mother recovered from her stroke and came back to her daily life. Since then, Sunjoo called her mother every day to check up on her. In 1995, her mother feels at home and fractured her hip joint. The day of the accident, the mother's line was busy all day long, as the mother had fallen on the way to answer the telephone. In spite of operation, the mother failed to recover her full mobility resulting in her needing assistance with daily tasks. Even though Sunjoo is only one of four children (one son and three daughters), she was the only one willing to care for her mother.

In 1997, Sunjoo's husband was fired from the company he worked for in the aftermath of the Asian financial crisis in 1997. The husband received an early retirement package in a lump sum, which ran out rapidly, for investment in starting up a new business living expenses, and the university tuition fees of the three children. The new company, however, became bankrupt after a short period, and her husband had to find another job. Sunjoo also had to seek part-time employment to contribute to earnings.

After she had cared for her mother alone for more than 10 years, Sunjoo heard about the implementation of the LTCI in 2008. She also heard that family caregivers could be paid for their work when they obtained a licence as a care helper. At first, she hesitated to obtain a care licence, but one of her friends encouraged her to take courses for a care helper's licence together, and she accepted it. At first, she doubted the concept of compensation for family caregiving. But she completed the courses and became a FCH who had a 'contract' with a care centre. 
Since her mother lived alone, Sunjoo and her family visit and care for the elderly woman every day. Although her formal care recognition is one and a half hours a day (7:00-8:30 am), she usually stays all morning long. Due to the mother's physical impediments, her daughter does all house chores, such as cooking, clothing, cleaning, laundry, as well as bathing her once a week with assistance from another care helper. Sunjoo’s children help her to care for the elderly, especially Sunjoo's son picks up Sunjoo every day, and sometimes he takes the grandmother for a drive.

Sunjoo is the second child and has one older brother and two younger sisters. However, the brother and the youngest neither care for the mother nor have contact with her. Only the third child (Sunjoo's younger sister) sends Sunjoo money to support the mother (about 50,000 KRW (about 50 USD) monthly). Incomes related to the mother comprise a basic pension benefit (200,000 KRW, about 200 USD), a payment for caregiving of the FCH (400,000 KRW, about 400 USD), and the third child's support (50,000 KRW, about 50 USD) while costs include food, medicine expenses public utility bills as well as pocket money.

Sunjoo manages both the incomes and the costs and gives only some pocket money to the mother directly. With regard to public pension benefits, Sunjoo's mother does not receive pension benefits through the national pension system (NPS) but does receive tax-based benefits (the basic pension) with200,000 KRW (about 200 USD) per month. Even though the mother knew about the payment for the FCH from the LTCI, she did not ask for the money.

Sunjoo took responsibility for her mother, even though she had not yet worked a full-time job long enough to pay the contribution for any insurance scheme. So far, she and her husband could not meet the requirements for the old age pension benefit (the NPS); she has paid the contribution for the NPS only for three years, and her husband has been low-paid worker ever since he lost his first job, meaning he is not entitled to the pension benefit or the unemployment benefit either. Moreover, they have burdened themselves not only with the elderly care but also with the support of their children, who have not yet married and could not afford to live independently. So, Sunjoo decided to open a small convenience store with her son. 


\section{Type B: The negotiators}

Sunjoo's case is representative of the type "negotiators" and I found four notable points in her story. Firstly, Sunjoo's story implies that care can be understood not only as labour for a dependent one but also as a relationship between caregiver and care-receiver. Sunjoo is willing to care for her mother and to let her stay at home instead of a care facility. Through caring for her mother, Sunjoo builds a better relationship and emotional bond with her previously estranged mother. Compared to other adult children interviewees, Sunjoo was more likely to be in conflict with her mother because of the family history of her mother having left Sunjoo and her siblings during their childhoods. Indeed, Sunjoo's brother and sisters refuse contact with their mother. Unlike her siblings, she took caring for her mother as a chance to recover from the negative memories. As mentioned above, even if she knew her mother had left her, she never lost her feelings for her mother and tried to empathise with her situation. Apart from that, she improves her own self-esteem by caring for her mother by herself. These experiences show that caring for others can give people chances for recovery and self-growth. Even though there are conflicts between her and her siblings, and the conflicts hurt her a lot, the decision itself was not affected by these conflicts.

Secondly, Sunjoo has cared for her mother for almost twenty years. She started to look after her before the introduction of the LTCI, which means she has experienced a transition in her position from informal to semi-formal care worker. Moreover, she experienced changes in recognition hours for in-home care services offered by FCHs as well as in recording systems from paperwork to an electronic device, the RFID. By tracing Sunjoo's experiences across these twenty years, we can better comprehend how a family caregiver copes with new situations and changes regarding the public care service provisions. The coping strategies of family carers have implications for the relationship between formal and informal elder care. From Sunjoo's case, we can see that new formal care provision does not replace all existing informal care. Instead, informal care is complemented by the new public care provision.

Thirdly, Sunjoo has had relatively few problems when it comes to entering the labour market. She stayed at home as a housewife and has cared for her three children and her frail mother. She sometimes worked in a temporary position, but it is hard to say that she works there for a living. Rather, she works at the convenience store her son runs as an unpaid family worker. Even though she has a working contract with a care centre, she only works 
as a FCH for her frail mother. In this situation, Sunjoo's care helper licence and the working contract are just a procedure she uses to access compensation for the care work she does anyway. From Sunjoo's story, it is clear that the meaning and characteristics of caregiving as a FCH can vary in the status and intention of the carer. This could imply that there is a paid care work which posits beyond in legal definition of labour.

Lastly, even though FCHs have a working contract with a care centre under which they will be paid, they are not under the control or supervision of a social worker in the care centre. Thus, FCHs are in between a service user and a service provider. As new social risks emerge, new kinds of public provisions and arrangements are also needed. Sunjoo’s story has implications for how we understand care work which cannot be categorised as employment in the labour market.

\subsection{Type C: The Partners}

This section aims to investigate the experiences of caring for an older spouse by analysing Jina's story. Jina represents eight interviewees, whose type I call "the partners". Jina's case exemplifies type $\mathrm{C}$, the partners who take care of their spouses but cannot expect the same support from other family members like, for example, their adult children and face a lack of support from the state. I found almost all spouses of this study to represent the type of "the partners". Today's spouse carers are different from those of older generations as they are generally better educated and have more opportunities for paid work, whereas older generations were less educated and rarely went into the labour market. This not only leads to a different labour market experience but also to a different perception of oneself, one's capabilities, one's role in the family, and one's interactions with others and the world. In this sense, the characteristics of the spouse carers in modern Korea - their experiences, perception, and living strategies - show a different picture from their portrayal in both Western literature and past research conducted in Korea.

Jina started to care for her husband in 2012 when she was at 60 when he was diagnosed with Alzheimer's, and she became a licenced family care helper (FCH) in 2014. She lives with her husband, they have one son and one daughter, both married and living separately from their parents. The section starts by providing a brief interview context in section 5.4.1, followed by the interviewee's self-presentation (section 5.4.2) and a case reconstruction of 
Jina's life history (section 5.4.3). It then provides a structural summary in section 5.4.4, as well as more general discussions on "the partners".

\subsubsection{Interview context}

I met Jina at a care centre, where she was enrolled as a FCH. While I mainly recruited interviewees through introductions from the social workers of a care centre, the recruiting process was different in this case: I had an opportunity to explain my research interest in front of family care helpers who attended an education programme held by their care centre. Jina gave me her phone number there, and, after the meeting, she and I walked to the bus stop together on our way home. She told me that she agreed to be interviewed because I reminded her of her daughter, and she had already participated in several surveys and clinical trials for her husband before.

Two days later, I visited her at her home, a second-floor flat in a multi-family residential building where she was living with her husband. I tried to interview with her somewhere separate without her husband for reasons of privacy, but she wanted to watch her husband during the interview since she has to look after him and he could not understand the interview due to his cognitive impairment. Indeed, he made no comments or responses during the interview. Instead, he wandered around the living room and tried to touch a hot kettle in the kitchen so Jina had to keep him from danger. The interview lasted for about two hours.

\subsubsection{Self-presentation}

Before I asked my opening question aimed at generating Jina's main narration of becoming a FCH, I tried to make some small talk to break the ice. There she gave me general information about her children and grandchildren. Although the interview lasted about two hours, the initial narration of the interviewee was relatively short (about 3:00 minutes). This can be due to the fact that my first 'open' question was formulated in a rather specific manner like "can you tell me about your husband's disease freely”, instead of asking about her general life story. Moreover, she formulated her answer by repeating my question like “about his disease”. I intended to encourage her general narration with the word 'freely', but it ended in failure with her answer repeating my question.

She began her narration with her husband's unhealthy lifestyle and a chronicle of the diseases he had suffered from since he had been in his forties in the late 1980s. She listed 
how many diseases and operations her husband had with explanations of his unhealthy habits like binge drinking and heavy smoking on a daily based. The initial narration was very brief and short, and the main text sort were descriptions. One can interpret her presentation in various ways: 1) she tried to convey a detailed and specific answer to the question by starting from the husband's first signs of diseases in his forties, or 2) she tried to hide or avoid talking about his present health condition, or 3) she linked the present health condition of her husband to his unhealthy lifestyle.

With these hypotheses in mind, I asked her to talk more about her husband in the second phase of the interview. She explained her husband had worked as an employee driving heavy vehicles before he was diagnosed with the Alzheimer disease in 2012, then she moved back in the timeline to the 1990s and started to talk about her family business. She explained the husband's business and her hardships to run the family business in many details, but taking a closer look at this section reveals the text sort still to be a description rather than a narration.

After providing several descriptions, she went back to the topic of her husband's disease, the onset of the Alzheimer's disease (at 00:09:00 minutes of the record). Unlike her former descriptive explanations, she remembered the episode so vividly, even down to the date and the chain of events of the day, that this can be classified as the initial narration she offers. She begins the story by saying "I realised that dementia came to him", and first explaining their general living status; for example, they had two apartments at that time, and her husband and son earned relatively high salaries, her daughter pregnant. My impression of her narration was that she was happy with her life in that period, at least until February $28^{\text {th }}$ before realising her husband's symptoms. This discordance between the topic (onset of the disease) and the specific explanations (stable and affluent living status) could be interpreted as that the onset of the disease marks a major changing point for her in her life.

Apart from the topic of the diseases, housing (or more specifically apartments) were another main theme of her narration. When she remembered a certain period, she always mentioned where she lived at that time and offered details on the dimensions of the flat and the district of the city it was located in. This mention on 'the apartment' in different contexts, gave me an impression that her comments on the apartment stood for her way of living and establishing property. Jina experienced several moves due to financial reasons 
and family conflict; therefore, the place where she lived was her criteria for judging whether her life was stable, and this stability of life was also linked closely to her idea of living in retirement, and caregiving.

Throughout Jina's Interview, I had the impression that she tried to present herself as a person who faced many challenging situations but attempted to overcome those challenges by herself. After long and repeated descriptions about the apartments her family used to live in, she told the story about how she decided to become a FCH. She explained that he had to solve two different but linked problems. She argued that she needed a new resource to supplement the suspension of her income by explaining the various income sources in her old age. Besides the shortage of money, Jina said that caring for her husband herself was better than having others care for him, or his entering a nursing home. In summary, her interest in presentation can be formulated as the following thematic field: "caring as the way of independent living with my partner given unstable and tough situations in oldage”.

\subsubsection{Reconstruction of Jina's life story}

\section{Educational background as a symbol}

Jina was born in a small country village in 1952. She has three younger sisters and one brother and is the oldest of five siblings. According to her explanation, her family was welloff, and it was thus not difficult for her to finish middle school. However, she experienced a challenging situation regarding her high school education and did not get to complete a high school diploma until 2002 when she was already 50 years old.

The earliest moment she spoke of during the interview was the end of her middle school life when it was time to map out the future course of her (working) life. At the end of middle school, she planned to enter a women's commercial high school, as she thought studying there would give her an opportunity to get a well-paid job (such as working as a bank clerk). However, she failed an entrance exam for the high school she wanted the most, and her second try for another high school was also unsuccessful. She said she could have studied at another non-commercial standard high school, but she thought that would not be useful 
for getting a job, and her mother forced her to earn money instead of pursuing her education.

We need to note that most girls in the mid-1960s had limited educational opportunities due to the ideals of patriarchal Confucianism that was very highly regarded at the time and discriminated against girls in education and consequentially in the labour market. Instead, they were asked to help their family by earning money as a secondary earner, or by managing household chores (Lee Hyunju, 2015: 158). Jina was frustrated at not being able to go to high school because her family could have afforded her education; thus, she thinks dominant gendered norms in the 1960s, which did not value female education, played a part in hampering her educational aspirations.

After her failure to enter commercial high school, Jina stayed at home for several years and helped her family. Although Jina failed to enter higher education, one of her younger sisters passed an exam for a women's commercial high school in Seoul, and Jina moved there with her. At first, they lived in the house of their aunt (a sister of her father), but then they moved out. Jina worked in a factory, mainly in the light industry sector, on the product lines of electronic or pharmaceutical companies.

While working full-time at a factory in Seoul, she also tried to obtain a secondary education certificate through a distance learning course. When asked about her education, she mentioned why she tried to further it:

"I was definitely eager to have a graduation certification. The reason was that, when I applied for a job, I was definitely keen to have that certification, as an educational background was essential (interviewer: yes, I see). The educational background was critical, so I was definitely eager to have it (interviewer: I see). If I had finished women's high school, then I would have married a good person." 60

With her comments, one can interpret that her decision to move to Seoul was initially to earn money for a living, but she had another attempt at improving her educational level. It is notable that she thought her educational background had not only to affect at her chances of getting a well-paid job but also her chances of finding a partner, which suggests the

\footnotetext{
60졸업장을 꼭 갖고 싶더라고. 졸업장이 왜 필요하냐면, 취직할 때, 졸업장이 꼭 갖고 싶더라고. 학력이 중요하니깐.(그쵸, 맞아요) 학력이 중요해서, 그게 꼭 갖고 싶었었고, (그게) 내가 그때 여고만 나왔어요(네) 좋은 사람 만나서 시집 갔을텐데(919-921)
} 
meaning of education could vary by gender. Jun Hee-Kyung (2013) argued that even if women had a higher level of education, they could not enter the paid labour market in the same way as men. Therefore, while men could use their education level and skills for vocational achievements and social status, women were more likely to use it as part of their matchmaking. Indeed, the economic participation ratio for married women with a high level of education stayed low in Korea until the 1980s, since a higher level of education for women was considered as a resource, symbolising expectations and the ability to attain a higher social status (Park Mee-Hae 1991; Min Kyung-Hee 2002). Thus, education itself did not lead women in Korea to participate in economic activities (Park Kyung-Suk and Young-Hye Kim 2005, 136). Jina's account shows that she planned to attain a higher social status by obtaining a high school certificate as an opportunity to obtain either a well-paid job or a partner in a higher social status.

Jina enrolled in high school for distance learning while working in Seoul but had to give up when she married her husband, whom she met through a matchmaker, and moved to another city. After her marriage, she mainly stayed home to take care of her two children, but from time to time she worked at a factory in the light industry as a secondary earner (when needed). She suspended her studies for more than 25 years, but finally completed the course and got her certificate in 2002 when she was 50 years old. Even if she had not altered her past career or partner through her late high school graduation, she was able to get a part-time position at a day-care centre that required a resume. She also mentioned her high school graduation to her son-in-law when her daughter married:

"I told my son-in-law everything since he might wonder about it. I opened up about everything."61

Her account implied that she thought her educational background symbolised a social status that was necessary to satisfy her daughter's extended family-in-law, therefore, she tried to obtain the high school graduation after being fixed in her former social status for so long.

\section{Married life as a member of an extended family}

As in the course of her education, Jina also experienced challenges in her marriage. She is the oldest daughter-in-law in an extended family and is therefore faced with obligations

61나는 사위도 궁금해할 것 같아서 다 이야기 했어. 다 털어놔 나는 (939) 
and responsibility within traditional family culture. However, she negotiated the responsibilities with her family-in-law instead of submitting to traditional family culture.

Jina met her husband through a matchmaker and they got married in 1976. Her husband was the oldest of eight siblings, which means Jina became the eldest daughter-in-law and lived with her new extended family, including her parents-in-law and three unmarried brothers-in-law. She mentioned severe conflicts, especially with one of the brothers-in-law (her husband's younger brother). The situation of co-habitation sharpened this conflict as they had to live together for several years and Jina had to manage most of the family chores, including cleaning and preparing meals. She had trouble with the brother-in-law regarding financial issues. When she lived with the family-in-law, the brother-in-law's business failed, resulting in bankruptcy; the family had to sell their house. She explained the financial trouble caused by her brother-in-law:

"The brother-in-law asked for a mortgage on the house where the family lived (interviewer: hmm). I thus allowed him to take out a mortgage on the house (interviewer: yes); a couple of months later, the house was foreclosed (interviewer: $\mathrm{hmm}$ ). The house in district D (interviewer: I see, you lost the house), it might have been 40 pyeong ${ }^{62,}$ but it was seized." 63

To save the house from creditors and in order to be able to keep living there, they had to ask a friend to take over the house on the condition that Jina's family paid off the debt and bought the house back from the friend. Since the house in which Jina's family-in-law lived was the only property they had, its loss was a severe blow. However, Jina talks about the event in the context of overcoming hardship:

"At that time, we could afford it, since I worked and earned money (interviewer: yes). We could provide for ourselves, kind of, and we borrowed money from a bank again, so we bought back the house again - the same house. Then, we

\footnotetext{
${ }^{62}$ The pyeong is a traditional Korean unit of measure used to indicate space, especially for land and housing. Forty pyeong is about 132.2 square metres.

${ }^{63}$ 금방 시동생이, 와리깡 해달라고 해가지고(음음) 그 집 담보로 어음 와리깡 해줬는데(네) 몇 개월 지나니깐 차압이 들어온 거야(음) 도화동 집에(네, 날라갔군요) 사십 몇 평인 데, 대지가 한 사십 한 오평정도 되는데. 그게 이제 차압이 들어오니깐(534-536)
} 
lived there; later, we could borrow money again and started to run a business in 1991 or 1992."64

Jina recalls that she and her husband paid off their brother's debt and bought the house back by themselves. Moreover, they could raise a loan for capital for a new business secured on the home they had bought back. This episode, however, deepened the conflict between Jina and the family-in-law; thus, Jina and her family lived separately from their extended family from there on.

Another topic of conflict between herself and her family-in-law Jina touched on in her accounts was the care she was expected to provide for her frail mother-in-law. From 1996 on, Jina's mother-in-law was in need of care due to hip fracture and diabetic complications. To care for her mother-in-law, Jina's family moved into a 38-pyeong (about 125-square metre) four-room apartment and lived with her mother-in-law again, but they could not afford the apartment. Jina was working part-time at a factory nearby to pay back interest on the debt incurred in establishing the family business, so she could not commit to caring for her mother-in-law at all times. In addition to this, she also did not have a good relationship with her mother-in-law before and did not jump at the chance to care for her.

However, Jina and her husband accepted to care for the mother-in-law, respecting the traditional obligation of the oldest son. One of Jina's sisters-in-law, her husband's younger sister, helped care for the mother by hiring a private care assistant. This left Jina trying to balance two roles: as a secondary earner and as the first daughter-in-law offering care based on Korean traditional care culture, compromising on this way of care by sharing the burden with her siblings-in-law (or more precisely the care assistant paid by them).

After Jina’s mother-in-law died in the late 1990s, the family moved into a smaller house due to their economic difficulties. When her husband's business got worse, he decided to shut it down and return to working as an employee driving heavy vehicles. Jina mentioned that the salaries at this time were higher than the income they had received while running the family business. As her husband was receiving a relatively high and stable salary, Jina’s household had spare money to invest again.

\footnotetext{
64우리가 좀 여유가 있다 보니깐, 그땐 사회생활, 직장생활 했어요(네) 여유가 이제 조금 이제 은행 대출도 받고 이래서, 다시 이래서, 다시 그 집을 두 번을 산 거야 그 집을,(응) 그 집을 두 번을 산거야 그래가지고 그 집 살다가 거기서 인제 대출받아가지고 사업을 시작한 거지 구십 일, 이년도(538-540)
} 


\section{The role of an apartment: A place to roost, foundation money, or an investment object?}

When she recounted her life history, the events she experienced were constructed according to her move from one house to another. Jina repeatedly mentions the places where she lived in detail: for instance, ' $a$ 17-pyeong (about 56 square metres) apartment ${ }^{65}$ in $\mathrm{K}$ village of $J$ district (453)', 'a 40-pyeong (about 132 square metres) house in D district (537)', 'a 38pyeong (about 123 square metres) $H$ apartment in $Y$ district (550)'. Her explanations of the places where her family lived included the name of districts, the brands of the apartment, and the size of the house, and her account implied the multifaceted role of apartments in Korea as living places, as foundation money for establishing a business, as an investment, or as a critical security measure for retirement.

Firstly, housing was important to Jina because she experienced insecure living conditions due to financial difficulties and family conflicts. The changes in Jina's family living arrangements were often connected to family issues, like conflicts with family-in-law, financial trouble caused by other family members in the extended family, or the care needs of their old parent. She explained that she had moved at least seven times after her marriage whenever she faced incidents regarding economic and family issues. After her marriage, she lived with her family-in-law for years but repeatedly begged her husband and parentsin-law to separate their living arrangements due to persistent conflicts. Her suggestion was accepted after she had several arguments with her mother-in-law and her second brotherin-law; Jina's nuclear family (Jina, her husband, and their two children) could then move out in 1980. However, the family had to come together again to care for Jina's frail motherin-law. Therefore, the continuous change in living arrangements during her life course could be an indication of the instability of her life in general.

Secondly, a house could be seen as a property used as a foundation for starting up a family business. Although Jina had a hard time in terms of housing and rent, she linked her time of affluence with her housing situation as well. As mentioned in the previous section, Jina's family could apply for a loan for their family business through a mortgage on their house:

\footnotetext{
${ }^{65}$ The term 'apartment' refers to multiplex housing with more than five stories, including a dormitory (KOSIS, 2017).
} 
"We could borrow money again and started to run a business in 1991 or 1992." 66

Moreover, the apartment was not only a fund-raising resource for Jina's family business; it was also a means of investment. For several years before Jina's husband was diagnosed with Alzheimer's disease, her family earned enough money to pay off all their debt and could finally spare money. Jina's family, therefore, considered investing their money, and their son urged Jina to sign a contract to buy an apartment as a means of investment:

"In the late 2000s, in 2007 or 2008, my son had about ten million won and tried to buy another apartment. My son bought the $X$ apartment with future profit in mind, so he thought we could also do it since my husband had a salary and a new apartment market began to open at that time." 67

In this sense, the meaning of an apartment could be both positive and negative for Jina. At her son's suggestion, Jina signed a contract to buy an apartment and applied for a mortgage. She thought her family could afford to pay the rest later because her husband earned enough money in his job operating heavy vehicles (like the forklift of an excavator). However, her husband was diagnosed with Alzheimer's in 2012 and could neither work nor pay off the rest of the mortgage. Jina's family had to move out of the apartment into a tiny, monthlyleased flat in a three-storey multiplex housing block. Because of the many different kinds of housing Jina experienced, it made her judge one's stability of living by one's living arrangements:

"I do not think it is good to move around in my old age, but when I go for a walk to a park, there are many grandmas who have a lease. I don't know exactly whether they pay monthly rent or Jeonse, ${ }^{68}$ but many have a lease (interviewer: I see) - a lot." 69

\footnotetext{
66거기서 인제 대출 받아가지고 사업을 시작한 거지 구십 일, 이년도(540)

67 이천년도 후반에, 이천년 한 7,8 년도에는, 돈 천 만원만 있으면, 그낭 가서 아파트를 자꾸 살라 그러더라고. 지가 인제 자이아파트 살아 보니깐, 돈이 얼마 조금 들어가서 이렇게 남았는데, 이생각을 하면서, 그래서 청라로 아빠 돈 벌고 이러니깐, 그때 인제 막 입주가 시작될 때거든(125-127)

${ }^{68}$ Jeonse is one of the forms of payment made to a landlord in Korea. As opposed to monthly rent, Jeonse requires the lessee to make a key money deposit of about two-thirds of the total cost of the leased property in place of the monthly rent. The key money deposit is returned when the rental contract expires. 
Jina's account implies that housing is one of the most important factors for a secure life in old age. The levels of social security (such as old age pensions and public housing for the elderly) have not yet been established for the present elderly in Korea. They thus have to make provisions for their retirement individually. Without public transfers, the elderly in Korea have to depend on support from their children or from the property they have acquired while working. In Korea, real estate accounted for $69.8 \%$ of total household property in 2017 (Kostat 2017). Ownership of a house is therefore considered an important indicator of old age financial security (Jun Sang-In 2007).

Meanwhile, Jina mentioned the brands of the apartments she had known in parallel with their size and the districts in which she used to live. Whereas apartments have for decades been named after the major company planning and constructing them, a trend emerged in the 2000s of branding apartments like a commercial product (Jun Sang-In 2007: 18).

Several studies on preferences of living in branded apartments in Korea have concluded that Koreans perceive apartment brands to pertain to values like well-being, a luxurious lifestyle, and family happiness (Chae Wan 2004; Jun Sang-In 2007). Lee Dong-Yeon (2010) also points out that branding apartments have become a symbolic capital in Korea. Jina's comments on the brands of the apartments she lived in could be understood as a reflection on the social status and achievement she perceived herself when she lived there.

To sum it up, the apartments Jina mentioned during the interview showed her way of living and establishing her property. It also linked with her idea of living in retirement. In Korea, owning a house is considered as the only way for the formation of property. This is due to prevailing perceptions that house prices are supposed to increase; thus, houses are gradually becoming places that are not only for the living but also for investment. This perception has driven housing expenses to very high levels. Jina concluded a contract to buy an apartment in 2008 when her family income was stable and relatively high compared to before, but her plans were thwarted by her husband's unexpected health issues.

\section{Alzheimer's disease revealed by an accident}

Jina's husband was diagnosed with Alzheimer's disease in 2012 when he was 70 years old. Before he was diagnosed, he had suffered from other diseases, including type 2 diabetes, since his forties, and had had two heart attacks in his late fifties. When I asked her about 
her husband's condition, she started to talk about the diseases he had been diagnosed with his forties:

"Even though he had diabetes, he did not care for his diet or his medication. He neither took drugs nor managed his diet. He just ate anything he wanted, like alcohol, and therefore he got a myocardial infarction in 2002."70

Jina's account suggests that her husband's condition has gradually declined, but she never noticed his Alzheimer's disease until the disease was inadvertently revealed by a traffic accident. She remembered the episode vividly, even down to the date and the events of the day:

"Well, my son earned some money, and we did not spend too much money, so we could pay off our debt, too. My husband ran a business alone, and it was on 28 February 2012 that he came home drunk. It seemed to me that he had got drunk (interviewer: I see), and then he had fallen asleep (...) When we woke up the next day, I got a phone call (interviewer: okay), the phone call was not for me. My son phoned me and said, 'What happened to dad?' and I answered, 'Dad came home last night and slept' (interviewer: I see), but my son told me he had had a call from the police for a hit-and-run." 71

Her account suggests that, when Jina heard about her husband's hit-and-run, her family was financially stable compared to other phases they had endured in the past. Both her two children were married and in stable jobs, and Jina’s husband was receiving a relatively high salary for operating heavy vehicles. At the time of the accident, Jina's husband was 70 years old but still working. The rate of labour for people over the age of 65 in Korea was 70.9\% in 2015, a much higher rate compared to other Organization for Economic Cooperation and Development (OECD) countries. In fact, Korea was ranked the top for labour over the age of 75 for four consecutive years from 2013 to 2016 (OECD 2018). Such a high percentage of elderly economic participation has been understood as a strategy for

\footnotetext{
70 당뇨가 있는데(네) 당뇨병을 않으면서도, 음식이라던가, 약이라던가 이런 거를 먹지를 않았어요. 약도 안먹고, 음식도 그냥 자기 먹고 싶은 거 술 같은거 먹고 싶은 것 다 먹고. 그리고 2002 년도에 갑작 심근경색이 와서, 그때서부터(41-43)

71 생활이 좀 그때는 좀 아들 벌고 나가는 거 없으니깐, 좀 빚도 갚고 뭐 이러다 보니깐, 남편이 인제 혼자 사업해서 2012 년에 2 월 28 일날 왔는데, 술이 조금 취했어 내가 볼 때는(네) 취했는데, 그냥 와서 자더라고, 그때 사위하고 딸도 왔었어(아, 가족이 다 모였는데) 임신이, 임신이 좀 된, 3 개월인가 이렇 게 됐는 데, 왔는 데, 그 이튿날 자고 일어나니깐, 전화가 왔어(네) 전화가 나한테 온 게 아니라 우리 아들한테 전화가 와가지고 ‘아빠 어떻게 된거냐고?' 그래서 '아빠 어저께 저녁에 들어와서, 잤다고' 그랬더니(네) 경찰서에서 전화왔대, 뺑소니로(94-99)
} 
coping with the shortage of old age income instead of active ageing ${ }^{72}$ (Yoon Minseok 2016: 4; Hwang Sun-Jae and Cheong-Seok Kim 2013, 221).

They went to the police and verified the accuracy of the report. The scene of the husband's accident had entirely been recorded from surveillance cameras on the highway, and he had to pay 7,000 USD as a settlement for his hit-and-run. Although the case was closed by reaching an agreement between her husband and the owner of the damaged car, he never did remember or accept that the accident had occurred. His denial of the accident confused Jina, and she urged her husband to get a check-up for dementia at the hospital. After several tests, he was diagnosed with Alzheimer's disease. He had to quit his highly-paid job, and they gave up their new apartment and moved into a smaller flat, with a much lower monthly rent. The abrupt changes due to her husband's health affected not only their living conditions and caused an interruption to his career, but also destroyed the perspectives and plans they had for the future.

With increasing, age workers are more likely to lose labour capacity due to chronic illness, and the suspension of income caused by labour incapacity is more likely to affect the life of the elderly. Old age income resources in Korea consist of public transfers, private transfers, and labour income. Public income refers to publicly constructed income, such as old age pension benefits or tax-funded income supports, while private income in Korea mainly comes from the support of adult children in the sense of obligation. Changes in family structure and women's participation in the labour market have led to more couples living in a two-person household in old age, without adult children supporting them or caring for them. Against this Background, labour income even in old age has become more and more important.

Although her husband quit his position in the heavy vehicle company, Jina let him work part-time in the public sector in 2012, since she thought small paid activities were better for his health and the family income. She also worked as an assistant at a kindergarten nearby in 2014 at 62 :

\footnotetext{
${ }^{72}$ See more details in Chapter II, section 2.3.
} 
"He could get 200,000 won ${ }^{73}$ monthly when he participated in community welfare centre programmes like picking up waste at the market (interviewer: was it something like public service employment?). Yes, yes, public service employment; maybe he went there twice a week (interviewer: okay) for two hours (interviewer: yes). Even though he was diagnosed with dementia, it got better when he went there and pursued some activities (interviewer: I see). I also worked at a kindergarten (interviewer: okay). I went to the kindergarten and earned 400,000 won monthly." ${ }^{74}$

With this arrangement, Jina took action in the face of a challenging situation, and this showed Jina trying to live independently instead of relying on the support of her adult children.

\section{Becoming a FCH}

Since being diagnosed with Alzheimer's disease in 2012, the husband's health condition worsened progressively, until neither he nor Jina could continue to work. The husband could not manage the tasks of his public-funded job programmes any more, and Jina had to care for her husband, who could not be left at home alone. Therefore, some of the neighbours recommended she become a yoyangbohosa (요양보호사, care helper) in the long-term care insurance (LTCI). According to her neighbours' recommendation, there were some advantages when family members cared for the elderly as a yoyangbohosa:

\footnotetext{
"Someone told me to care for my husband through a licence as a yoyangbohosa, as it would be better (interviewer: okay). I knew about the licence before; I did not think that I could get it (interviewer: ah okay). And since last March we have almost fallen into poverty." 75
}

Jina's decision to become a FCH can be seen as a strategy for coping with two major problems. Her family needed some source of income, but she was the only one who could care for her husband. The word 'better' in Jina's account implies that to become a FCH was better for the family income and her husband's condition. In this situation, her licence

\footnotetext{
${ }^{73}$ About 200 USD.

74그 복지관에서 하는(네) 그 여기 뭐야, 도매시장에 가서 이렇게 저 뭐야 휴지 같은 거 주우면(네) 한 달에 20 만원씩 줘요. (공공근로사업 같은 거군요) 응응, 공공근로사업, 1 주일에 두 번인가 이렇게 가면(네) 두 시간씩 하고(네) 치매라는 병을 받았어도, 그런 걸 가서 좀 활동을 하면(그럼요) 좀 좋을까 해서. 나는 또 유치원에, 유치원에 나갔었거든요. (아) 유치원에 나가서 한 40 만원씩 벌었거든요 (143-146)

75누가 그래, 요양보호사를 따서, 이렇게 아저씨 집에서 관리하라고 그래, 그게 더 낫다고(응) 그래서, '어, 그게 있구나' 하구서, 그게 그전부터 있는 거를 알았는데, 내가 막상 자격증을 따야 되겠다고 생각을 못했어요(아, 네) 그래서 작년에는

3 월달부터 돈도 진짜 빠듯빠듯 하거든 (195-198)
} 
as a yoyangbohosa (요양보호사, family care helper) functioned as a way to brig earning money into accord with caring for her husband. Even though she says she was willing to care for her husband regardless of the licence and payments.

To qualify as a care helper, Jina enrolled in a care helper education centre. The family caregivers who get the care helper licence to care for a family member only have to complete all courses as though they intend to work as a care helper for non-familial recipients of the LTCI programme for a living. The care helper licence is a national certification through a state exam, which requires taking courses, undergoing practice hours, and performing field training. To be able to take these courses, Jina had to register for the licence at an education centre. She paid 500 USD in total for the courses, which ran from 9 am to 5 pm every weekday for a month. When asked about the contents of the courses, she felt it was too comprehensive for her because she only needed the knowledge and skills for caring for her husband. However, she had to take all the courses to qualify for the licence. Indeed, there is no separate certificate for FCHs. They thus have to follow the same steps to qualification as non-familial care helpers

In addition to mastering the contents of the course, Jina faced the problem that she did not have anyone to look after her demented husband while she was attending classes and so he had to stay home alone. This made Jina worry for his safety:

\section{"After my course, I was unable to attend social events with other trainees, and I had to come home in a hurry because of my husband. I was wondering (interviewer: ah, okay) how he was, or if the gas stove was turned off correctly; for a variety of reasons, everything (interviewer: okay) and so on, I was worried." 76}

According to a study of Choi In-Hee (2012), 67\% of old spouse respondents answered that they had no one who could regularly help them to care for their spouse. Moreover, $16.3 \%$ of old spouse respondents had no supporter to whom they could turn for even temporary help. Jina's concerns about not being able to provide sufficient care for her husband lasted throughout her course sessions, but also during her field training and even her preparations for her exam as a care helper:

\footnotetext{
76끝나면 나는 남편 때문에 다른 거 다 취소하고 그냥 남편 핑계대고 빨리 올 수밖에 없어. 궁금하니깐.(아, 그럼 요)어, 남편이 또 어떻게 잘 있는지, 뭐 어떻게 가스불은 진짜 위험하진 않는지 여러가지로 다 (그럼요) 등등 걱정이 되잖아.(206-208)
} 
"I just studied there [in her living room], where it is cooler (interviewer: okay). In doing so, my husband was alone (interviewer: I see, you went to the education centre). He had been left alone since March in last year; he had been lying in a room (interviewer: ah, I see). I mean, he lay down all the time I was at the education centre. So, he kept lying down all the time (interviewer: okay). His condition got worse because he just kept lying down. He even refused to sit."77

Although Jina thought the contents of the education programme were too comprehensive, the qualification itself was worth it to become a formal carer. Unlike adult children, spouse caregivers often find it challenging to pass the exam to become a care helper.

During her training as a care helper in March, Jina also applied to the LTCI for her husband's entitlement to LTCI services. The application process for a recipient of the LTCI is separated from the qualification as a care helper. On the first assessment, the husband was assessed as Grade 3, which meant he could get in-home care services only. There are five different Grades in total; only severe cases with a first or Grade 3 assessment are entitled to get the cost of facility care services covered. The Grade assessments have to be renewed regularly, and the husband's Grades changed from Grade 3 to Grade 4 in July 2014, and again to Grade 3 in March 2015. Jina's experience in becoming a FCH shows that although many care helpers who offer in-home care services are family care helpers, there is neither a customised programme for family caregivers nor support for caregivers who need someone to care for their frail family member during their absence. Most family care helpers care for a family member only (Hong Sung-Wook 2011; Lee Yun-Kyung 2010); from the perspective of the family carers, it should, therefore, be enough to only get the specific knowledge they need regarding the illness or problems of their family members in order not to spend time and money ineffectively.

\section{Daily life: Care as an extension of marriage}

Traditionally, elderly care has been considered as the children's obligation based on Confucianism. Caring for parents has been regarded as one of the oldest son's obligations, but the oldest son's wife, the first daughter-in-law, commonly takes on the obligation instead of the son due to the gendered division of labour in traditional society. Until the

\footnotetext{
77 학원 끝나고 난 다음에는 저기서 시원한 데서 하다가 그낭(네) 그러다보니깐, 남편을 혼자있게 되었어, (그초 학원가는 시간이랑) 작년 3 월달부터 혼자 있으니깐, 계속 방에서 그냥 누워 있더라고(아,네) 그러니깐 내가 학원에 가있을 때, 계속 누워있어 버릇해서, 계속 누워 있더라고(네) 누워 있어서, 더 안좋구나 상태가, 이렇게 앉아서 보지도 못하고 (211-213)
} 
1990s, most family carers for the elderly in Korea were adult children, but the adult children caregivers have been decreasing in numbers due to changes in family structure, women's economic participation, and attitudes toward elder care. Instead, spousal caregiving has increased since the 1990s, and, paying respect to this, so has the research on the spousal carer (Lee Hyunju 2015: 143; Jung Kyung-Hee 2012).

Several studies have pointed out that daily life in spousal caregiving is different form caregiving offered by adult children (Choi In-Hee et al. 2012; Han Gyoung-Hae and SeoYoun Lee 2009) Firstly, spousal caregivers are likely to have closer bonds with the elderly based on their marriage life. Secondly, spouse caregivers are also usually closer to the age of the elderly they care for. Therefore, they are more likely to experience physical and mental burdens, including chronic illnesses and stress caused by social isolation and helplessness (Choi In-Hee and Jung-Hyun Kim 2013; Clyburn et al. 2000). In addition to these challenges, the shortage of income in old age also affects the experience of caring for the elderly. Compared to adult children, households of old couples are less likely to have a labour income; rather, they have to rely on other sources of income from public and private transfers. In this situation, the means of paying for care offered by spouse care helpers differs from that of payments for care offered by adult children care helpers.

Jina also thought to care for her husband was her responsibility:

"I have to do it because it is my work (interviewer: okay); it is my duty. As a married couple, we have lived together for a long time. We are inseparable (interviewer: I see), and I pity him for his illness, I pity him (interviewer: yes). I never thought about his dementia."78

Her statement on her responsibility for her husband indicates she has a different perception than was typical for spousal caregivers in the past. Studies, which analysed differences of care burdens according to family relations in 2000s in Korea, argued that spouse caregivers were likely to feel more burdened and stressed compared to adult children, since traditional care norms regarding children's obligation lingered in the country, and made the spouse caregivers perceive that they take over the children’s responsibilities (Han Kyung-Hae and Lee Seoyoun 2009; Cho Sung-Nam 2006). Unlike the past spouse caregivers in Korea in

\footnotetext{
78해야 되는 거죠. 내 업무니깐,(응응) 내 의무니깐, 부부하고 같이 남편하고 같이 한동안 살았으니깐, 살 맞대고 (그쵸) 그리고 불쌍하고 저런 병에 걸렸으니깐, 불쌍하잖아(응응) 생각지도 못했어 치매라는 생각은 (783-784)
} 
the 2000s, Jina did not feel she was forced to take a responsibility that should have been someone else's. Rather, she thought to care for the elderly was appropriate in the context of their married life and the affection for her husband. Also, her husband's disease evoked her sympathies based on the life they lived:

"I feel pity for him; he went through all sort of hardships (interviewer: okay), he kept on working, and he could not afford to clothe (interviewer: okay). He found working his only pleasure. It is a pity (interviewer: okay). I planned, when we got older, that we would stop working and travel somewhere after paying off our debts. Aww, we tried to take a trip, but..."79

As she described, Jina feels compassion for her husband due to the hardships he had to endure in life. As a partner who has lived together with him and has seen her husband's demanding life, Jina showed pity for him by mentioning an image of comfortable but unrealised peace in their old life. Her mention of an unrealised dream for the future reveals that she tried to understand her husband's situation which is linked with her future life as a spouse.

Since their children got married and moved out, only Jina and her husband were at home, which means she has to stay and care for him 24/7 alone. In addition to the home-visit care service, Jina uses other services compensated by the LTCI, like home-visit bathing and massage services for her husband. She tried to send her husband to a day-care centre, but she could not use the day-care service provided by the LTCI since her husband could not adjust to the atmosphere of the centre due to his Alzheimer's. Now, Jina's husband is not capable of undertaking the activities of daily life (ADLs) or the instrumental activities of daily life (IADLs) ${ }^{80}$ on his own. Jina helps him in everyday eating, bathing, dressing, going to the toilet, and taking medication. In addition to daily life, Jina has to manage medication

\footnotetext{
79불쌍하지, 젊었을 때 그렇게 고생 많이 하고(응) 오로지 일만하고. 옷 한 벌도 제대로 못입고 살고(응) 오로지 일하는 그 즐거움으로만 살고 그랬는데, 불쌍하지. (응) 나이 먹어서 어디 좀 돌아다녀, 고만, 돈 그만 벌고 어디 좀 돌아 다녀야지 했는데, 빚 다 갚고, 아우 돈 한 1 억만 벌면 어디 좀 돌아 다녀야지 했는데 (707-709)

${ }^{80}$ The activities of daily life (ADLs) and the instrumental activities of daily life (IADLs) are internationally used as a standard instrument for measurement for the functional status of the elderly. ADLs refer to essential daily self-care activities, including feeding oneself, bathing, dressing, grooming, IADLs are more about an independent life in a community such as cleaning and maintaining the house, managing money, shopping for groceries and necessities, taking prescribed medications. The LTCI uses ADLs and IADLs for an assessment for the entitlement to LTC services.
} 
and visiting schedules, as her husband has a variety of additional diseases including heart infarction, type 2 diabetes, and Parkinson's disease.

Jina has to manage all the care work for her husband alone, she, therefore, has physical burdens in caring for her husband, who is more likely to be exposed to risks and injury including falls, poisons, cuts, and burns. Therefore, caregivers for people with dementia have to watch for hazards. Jina also mentioned she could not take her eyes off her husband, and she even had to watch him during this interview. There are several studies focused on the spousal carer's physical burdens in cases of caring for their partner (Seomun Junhee and Kim Keunhong 2013; Han Gyoung-Hae and Lee Seo-Youn 2009). They find that, compared to adult children, spouses are more likely to be in old age themselves. Thus, they may not be able to manage all care work for their elderly due to own physical conditions and fragility, and the physical labour of care could cause problems like dizzy syndrome and musculoskeletal disorders. Jina, for example, also suffered from dizzy spells and degenerative arthritis. Unlike her commitment to her husband, she hesitates to care for herself. Except for taking traditional herbal medicines, she is reluctant to take diagnostic tests or call on medical assistance. When asked about her diagnostic test, she worried about the cost of the medical process. Even if her disease was diagnosed, she says, she could not afford the expense:

"when a disease is identified, I should take medicines regularly and can never stop thinking of the expense of the medication prescribed by a neurologist. Medicines given to neurology patients are usually expensive. Therefore, I could not go there, aw, due to economic reasons." 81

Indeed, according to a study by Choi In-Hee (2012), 45.3\% of the respondents who care for their spouses answered that they suffered ill health due to caregiving for their spouses. Also, a significant number of respondents suffered physical and emotional burdens, including fatigue (75\%), a lot of tension (65.7\%), and a desire to escape from their current situations (56.3\%).

In addition to physical burdens, Jina was also undergoing heavy emotional strains. As explained above, her husband was diagnosed with Alzheimer's disease in 2012, and his

\footnotetext{
81 좀 병명이 나타나면 매달매달 약먹어야 되잖아. 그 약값도 무시 못하더라고 신경과 쪽에는 신경과쪽은 또 약값이 비싸더라고. 뒷머리가 뻐근하고 이런데도, 아우 경제적으로 힘드니깐 못가는거야(488-490)
} 
physical and cognitive abilities have since deteriorated. He especially has problems with memory, language, judgement, sense of time, and orientation. Three years after his diagnosis, he could not recognise who his wife was anymore, and his cognitive impairment caused Jina emotional pain:

"He bathed in the morning, and I asked him who his bathing assistants were. He answered that they were his wives, even though I was right next to him (interviewer: I see). Everyone is his wife. If he knows nothing, what is the good of living like this (interviewer: okay)? He cannot recognise his son or his daughter. He can't even taste pleasure, pleasure." 82

Once her husband had reached this stage of delusion, she says she tried to focus more on her husband's misfortune instead of expressing her sad heart, since she fully recognised this were symptoms of his illness. She also told me that one could not enjoy life without a relationship with a beloved one. However, from her perspective on the enjoyment of life regarding the relationship, her pleasure connected with the relationship with her husband. Although she tried to understand and bear the situation with her husband, she not surprisingly still suffers from the emotional burden of her husband not recognizing her anymore.

In addition to the physical and emotional burdens, Jina has to deal with financial difficulties regarding her husbands' care. The financial challenges directly caused by suspensions of labour incomes, coupled with the low public transfers in old age in Korea and limited payments for family caregiving aggravated their financial situation. I want to call this situation a patchwork of incomes and analyse it in more detail in the following section.

\section{Limited recognition: A patchwork of incomes}

As mentioned in the previous section, Jina's decision on becoming a family care helper was meant to mitigating the financial difficulties of caring for her husband at home by herself. Therefore, it is interesting to know about Jina's perception and experiences regarding payments for the LTCI services she offered as a FCH. To analyse the payments she receives, I combine two different approaches. One approach is to focus on Jina's perception of the payments for FCHs compared to that for non-FCHs, as this enables to

\footnotetext{
82아까 목욕했거든 오전에? 그 목욕하는 선생님들한테 누구냐고 물어보니깐, 마누라래. 내가 옆에 있는데도(응) 다 마누라인거야. 그래서 차라리 아무것도 모르는데, 이렇게 살아서야 뭐할까(응) 아들도 모르고 딸도 모르고, 재미도 모르잖아. 재미(684-686)
} 
understand the meaning of the FCH programme in the LTCI. The other approach is to figure out how significant the payment for care for the elderly is in household incomes by examining the different kinds of income sources she has. This approach enables to understand the financial difficulties of the present elderly in Korea. I use the term, ' $a$ patchwork of incomes' to define the meaning of payments in a way that relates to both of the approaches. The payments for Jina's care work are much lower compared to that for non-FCHs. Therefore, the payments were not substantial enough to cover living expenses. Therefore, she had to gather different pieces of income sources from public transfers, private support, and labour income ${ }^{83}$.

For the first approach of comparing the payment for FCHs to that for non-FCHs, one needs to look at the official payment requirement and criteria for the FHCs. Like other FCHs, Jina obtained a care helper licence to receive compensation for caring for her husband from the LTCI. As described in Section 2.4 of becoming a FCH, a FCH has to take the full courses, undertake all the field training, and pass the exam for qualifying as a care helper, just like the non-familial care helpers. After qualification, a FCH should make a contract with a care centre, which manages and reports on care services to the LTCI. In the process of making this contract with the care centre, the family caregiver becomes a service provider in the LTCI system.

While the qualification process for FCHs is the same as that of non-FCHs, compensation standards vary according to whether the care helpers and the recipients are family or not. The LTCI pays a fee for the LTC services to service providers according to the hours the provider offers services. The recognition hours, in general, are counted for home-visit services offered by non-family care helpers up to four hours per day and thirty days per month. However, the recognition hours for the LTC services offered by family care helpers are lower, as their services offered are compensated only up to sixty minutes per day, twenty days per month.

By mentioning the costs of care for her husband, Jina maintained that the level of recognition of her in-home care services is too low. Indeed, she was paid about 200 USD

\footnotetext{
${ }^{83}$ See more details in $\mathrm{CH}$ II.
} 
monthly, and this was not enough to cover supplies like adult diapers for her husband's incontinence:

"I could earn an income about 200 USD (interviewer: okay), so I thought it was better to receive the expense of diapers (interviewer: umm, umm) since the cost of diapers could not be ignored, the expense of the diapers (interviewer: I see). So, for me, that 200 USD, it is too low an income as compensation for family care and protection (interviewer: it is). I heard it had been higher before; why has it decreased?"84

She could not understand the rationale behind the recognition for the hours of a FCH:

"I spend my 24 hours caring for my husband, first and last, all by my hands (interviewer: I see). I don't understand the differences." 85

When a person in common working age is in need of long-term care, the household is more likely to experience financial troubles, as the patient will have to give up participating in economic activities and one of the other family members has to care for them, meaning the family caregiver cannot work full time, either. In contrast, elderly care operates in a different context, because the elderly patient can be expected to already have retired from the labour market and become a pensioner. However, the number of elderly workers in Korea is extremely high compared to other OECD countries. According to a current OECD report (2018), a significant number of elderly Korean workers continue to work to avoid poverty and contribute to household income. This can be mainly traced down to two factors. One is that the public pension system in Korea has not yet been established. The other is Korean care culture being in a phase of transition.

"He receives little in pension benefits, about 300,000 KRW. So, I receive, then, now 600,000 KRW in total (interviewer: Is that the national pension plan?)"86

\footnotetext{
84요양보호사를 따서(센터에 있을 때) 센터에 있으니깐. 내가 수입이 그래도 한 20 만원씩 들어오니깐(네) 그래도 기저귀값이라도 들어오니깐 낫구나(음, 음) 귀저귀도 만만치 않아요. 귀저귀값(그러시겠네요.) 그래서 나는 그 20 만원 정도인 데, 가족요양보호가 너무 적은 것 같아 수입이(그쵸?) 그 전에는 많았다는데 왜 줄어들은 거야? (265-267)

85더군다나 24 시간 하고. 다 남편을 그낭 수발, 다 하나서부터 열까지 다 내 손으로 해야 하는 데, (맞아요)나는 그 차이가 나는 게 이해가 안가(271-272)

${ }^{86}$ The national pension plan refers to a contribution-based pension plan regulated according to the National Pension Act and run by the NPS. Unlike the national pension plan, the basic pension plan is tax-based,
} 
"The national pension plan and the basic pension plan (interviewer: the basic pension plan for the elderly) with 200,000 KRW, makes 300,000 in total." 87

From her comments, I got the impression that her household income is like a patchwork. To complement the shortage in household income, she receives an allowance from the basic national pension amounting to 200 USD monthly. Although receiving a variety of income resources, Jina's family finds this income is not enough to enjoy daily life. In addition to the benefits and allowances, her son pays the rent for his parents, and their daughter sometimes gives them spending money. Against this background, the basic pension benefits and payments from the LTCI play an important part in providing a living. Jina said she would have to work again if she was not receiving money for caring for her husband.

\section{Future perspectives}

Jina is ten years younger than her husband and never expected to care for him with Alzheimer's. When asked about her future without her husband, she has formed two different images and an idea of two different stages. One image is of her healthy life after completing her husband's care. Specifically, when asked about her close future, she assumed she would be old but still healthy. She mentioned active ageing:

"No, there are no options, but I have to consider. When my husband dies before me and I live alone, this situation may cause depression. Therefore, I will have new hobbies; anyway, I will not stay at home due to depression. What if I have depression?" 88

From her comment, she is worried about depression after her husband's death. Even if she feels physical and emotional burdens in caring for her husband, she assumes it could be worse without him. This implies that caring for her husband is to Jina not only work and duty but also an effort to maintain an independent family. There is, however, also another image what her future would look like if she got frail and could not live independently:

\footnotetext{
means-tested income support for the elderly. Korea launched a basic pension scheme on 1 January 2008. Both are public, old age income security systems.

87 저이도 돼 연금 조금 30 만원 정도 타고, 나도 30 만원 정도 타 그러니깐, 지금 60 , (국민연금 타세요?) 국민연금하고, 기초연금(기초노령연금) 20 만원하고 합해서 다 30 만원 정도 돼 (198-200) 
"Jina: Yes, I will meet many people (interviewer: okay). I have to be active (interviewer: okay); but I - what if I get sick, what should I do? I worry about that. (interviewer: If you cannot move freely?) Then I have to go to a nursing home." 89

She said that if she could not live independently, there were no options but to enter a nursing home. In general, women's life expectancy is higher than men's, and women often marry men who are older than them. Thus, they are likely to live alone without their husbands and have to look after themselves; in this sense, the problem of the ageing population and care for the elderly has a female face (Peng and Yeandle 2018), while men have spousal carers. Jina's comments show that, even though she has cared for her mother-in-law and now her husband based on obligations and affection, she does not want to ask her adult children to care for her in turn, She neither wants to force her children based on the obligations of Confucianism, nor does she seem to think that her adult children feel an emotional responsibility to care for her if necessary. There is another comment to be found in the interview on the way of care if she was to get frail and not be able to live independently anymore:

"Nowadays, old people are increasing in numbers (interviewer: okay). At least, for me, my father has me, but what about me if I have dementia? I don't want to become a burden to my children, but what should I do if I have dementia? Should I try to die by overdosing on pills? Sometimes, I have thought about it, and it's too frightening for me."90

This statement indicates that Jina feels very insecure about a future in which she might potentially be in need of care herself. She expresses that she does not want to impose on her children. The whole scenario seems to be so troubling and intimidating to her she even voices considerations of suicide. She feels that she is left without an agreeable choice. Unlike Western societies where social security for old age income and care supports for independent living of the elderly are well established by now, public care provisions for

\footnotetext{
89응, 사람을 많이 만나고(응) 자꾸 활동을 해야 돼.(응) 근 데 난 내가 아프면 '아우 어떻게 하지' 걱정이 돼 (막 거동이 아예 불편하시면 ) 그러면 요양원에 가야지(732-734)

90지금 노인세 대가 진짜 많아지고 있는 데(맞아요) 나부터도 진짜 나는, 남편은 내가 해주는데, 내가 만약에 치매하면, 아유 나는 어떻게 하지? 나는 진짜 애들한테 짐되기는 싫은데, 내가 진짜 치매라면 나는 어 떻게 하지? 약을 한주먹 먹고 죽어야 되나? 막 이런 생각도 어쩔 때는 가져. 나는 끔찍해 진짜 (300-303)
} 
the elderly in Korea have not yet been implemented to a sufficient extent to make up for the decline in traditional family-intern care arrangements (Kong Seonhee 2013).

\subsubsection{Structural features summary}

Jina was born in a small country village in 1952. She is the oldest of five siblings and has three younger sisters and one brother who is the youngest of the children... In 1968 when she graduated the middle school at 16 years of age, she planned to enter a women's commercial high school. However, she failed an entrance exam from the high school she wanted the most, and her second try for another high school was also unsuccessful. After her failure to enter commercial high school, Jina stayed at home for three years and helped her family. In 1971, one of her younger sisters passed an exam for a women's commercial high school in Seoul. Jina moved there together with the sister and lived in the house of their aunt (a sister of her father). Soon they, however, moved out from their aunt and lived two in a separate house. In Seoul, Jina worked in factories, mainly in the light industry sector, on the product lines of electronic or pharmaceutical companies. In her early twenties, Jina enrolled in high school through distance learning while working. In 1976, she had to give up these studies when she married her husband and moved to another city when she was 24 years.

Jina met her husband through a matchmaker and they married in 1976. Her husband was born in 1942 (he is 10 years older than Jina) and is the oldest of eight siblings. Jina lived with her extended family, including her parents-in-law and three unmarried brothers. In the late 1970s, she gave birth to a son (born 1977) and a daughter (born 1979). After her marriage, Jina worked on and off at a factory in the light industry sector at a secondary earner. One of Jina's brother-in-law (husband's younger brother) failed his business and the family had to sell their house (which was their only asset) to pay off his debts. Instead of moving out, Jina's family asked a friend to take over the house on the condition that Jina's family paid rents. In the mid-1980s, Jina and her husband were able to buy back the house from her friend. In the late 1980s, Jina, her husband and her children start to live separately from their extended family. The husband was diagnosed with type 2 diabetes in the late 1980s when he was in his late forties. In 1992, Jina and her husband started up a trucking transportation business. In 1994, Jina's mother-in-law was in need of care due to hip fracture and diabetic complications. Jina's family moved into a four-room together with her mother-in-law. Jina worked part-time at a factory nearby to pay back interest on the debt 
incurred in establishing the family business. In the autumn of 1996, Jina's mother-in-law died. In the same year, Jina's son entered a university. In the 1990s, Jina worked at a factory, and she first paid contributions to the NPS. In 1998, Jina's daughter entered a two-year junior college. When family business got worse, the family decided to shut it down; at that time Jina and her husband were 50 and 60 years respectively.

In 2002, Jina completed a high school diploma with 50 years of age. Her husband returned to work as an employee and earned a relatively high salary by driving heavy vehicles. In 2002, Jina's son got a good job at a large steel company and was paid a high salary. In those years, however, Jina's husband suffered two heart attacks but recovered after operations. In 2007, Jina's family bought an apartment for investment purposes and about a year later the first son got married, and the family applied for a loan to buy another apartment. Jina’s first granddaughter was born in 2009. Since 2008, Jina had worked at a day-care as an assistant - a job she would not have been able to get without her high school diploma. Jina's daughter married in 2011 when she was 32 years old.

In February of 2012, Jina's husband was indicted for a hit and run on a highway. Jina and her husband had to go to the police to verify the accuracy of the report. The scene of the husband's accident was entirely recorded by surveillance cameras on the highway. However, he claimed did not to remember the accident or accepted that it had occurred at all. Following this episode, she urged her husband to get a check-up for dementia at the hospital. After several tests, Jina's husband was diagnosed with Alzheimer's disease in 2012; Jina and husband were 60 and 70 years old respectively. He had to quit his highlypaid job. They also gave up their new apartment and moved into a smaller flat, with a much lower monthly rent.

After her husband quit his work, Jina let him work a little part-time job in the public sector. She still had her job as an assistant at a kindergarten. The husband's health worsened which meant neither he nor Jina could continue to work since he needed care and supervision 24/7. This resulted in major financial difficulties for the couple. Some of the neighbours recommended she become a FCH and in 2014 Jina enrolled in courses for care helper licence. For a month, she took a course from 9 am to $5 \mathrm{pm}$. During her training as a care helper, Jina also applied to the LTCI for her husband's entitlement for LTCI services. As a FCH, Jina is paid about 200,000 KRW (about 200 USD) for her formal care recognition of one and a half hours (7:00-8:30 am). Apart from the payment for family care, Jina and her 
husband receive pension benefits from the NPS (about 200 USD) and the basic pension (about 300 USD). And their son supports his parents with paying the rent for their flat.

\section{Type C: The Partners}

Jina's narrative shows her experiencing many challenging situations but also overcoming those challenges she faced throughout her life. She failed her high school entrance exams and endured severe conflicts with her family-in-law. Although she had a good relationship with her husband, her household suffered economic difficulties, and her focus in the apartments she lived in imply her need for a strategy to cope with her economic difficulties. Even if Jina has experienced trouble in her life, she actively responded and coped with it instead of passively accepting her situation. She moved to Seoul to work for the independent living and enrolled in long-distance learning at a high school. Due to her marriage, she gave up on a high school certificate at first, but finally finished her longdistance high school studies in 2002 when she was 50 years of age. In terms of conflicts with her family-in-law, she repeatedly appealed to move out and negotiated with her siblings-in-law about caring for her mother-in-law. She experienced challenging situations, but she overcame the difficulties and her family ran a family business by means of a loan.

Meanwhile, apartments played a pivotal role in her narrative - as a place to live, as a foundation for establishing a new family business, and as an investment object. Moreover, given the weak income replacement provided by the public pension plan of NPS and changes in family structure from extended families to elderly couples living alone, an apartment means a lot for independent living in retirement. Jina experienced several moves due to financial reasons and family conflict; therefore, the place where she lived became her main criteria for judging whether her life was stable. However, her perception on the apartment can not only be seen as that of an individual, but also a reflection of the social convention one should prepare an apartment for old life since public retirement income security is not enough to live independently. The need for disposable income in old age and the low level of housing stability in Korea mean that ownership of an apartment is fundamental in old age.

Jina's care for her husband can be understood in the context of this way of life. Firstly, Jina's decision to become a FCH can be interpreted as an active coping strategy in face of the complex care situation caused by the rapid decline of her husband's health. She had to solve two different but linked problems. One was that she needed a new resource to 
supplement the suspension of her income. Both she and her husband had to quit their job. In this situation, becoming a FCH was a possible income choice. Besides the shortage of money, Jina thought that caring for her husband herself was better than having others care for him, or his entering a nursing home.

However, as a caregiver, Jina needed help too, but she could not receive support from the LTCI or the government except through payment in for of wages for a licenced FCH. Here, Jina experienced challenges again, since while she felt to qualify as a care helper specifically for caring for her husband meant she would have needed only partial knowledge related to her husband conditions, but there is no educational programme for family care helpers specifically. Therefore, Jina had to take all courses in order to be compensated by the LTCI as a FCH. When she took the courses, however, her husband had to stay at home alone and there was no support in caring for him. While receiving no support from the LTCI as a family member of the recipient, Jina had to be under the control of the care centre as a service provider. She was tagged every day with RFID to report the beginning and the end of her services as a care helper; once a month she visited the care centre to report and attend a mandatory refresher workshop for care helpers. As a FCH, Jina was not viewed as a family member who needed support, but as a service provider who had to offer controlled quality service.

\subsection{Implications of the Typologies of FCHs}

By describing the Korean elderly care contexts in the previous chapter II, I differentiate between three broad sets of family care helpers' (FCHs) positions in negotiations with their family members on elderly care. This typology of the FCHs' positions is tentative, and the three types are not entirely mutually exclusive, and they illustrate all the issues discussed. Nevertheless, in discussing them in this study I will show how responsibility for elderly care is shared among family members.

This study arrived at three terms to represent these three responses of the FCHs on family care: 'complying with tradition', 'negotiation' and 'partnership'. Six respondents, almost all the first daughters-in-law, tried to comply with the traditional care culture and take on almost all responsibilities for the elderly care by themselves. Four handled it by negotiating elderly care with other family members; a further eight continued to develop a close 'partnership' with the elderly in the sense of solidarity between two individuals. The 
interview participants comprised seven spouses (six wives and one husband), four children (three daughters and one son), and seven in-laws (six daughters-in-law and one sister-inlaw). The majority of the interviewees were women, although one son and one husband cared for a family member as primary caregivers.

Almost all the respondents were married, but two respondents were single; one daughter who was caring for her frail mother was unmarried, and one sister-in-law who was caring for her frail brother-in-law (the husband of her sister) was bereaved. Those who were complying with the traditional care culture tended to experience caring as a source of stress due to the strong moral obligation required to handle it alone, while for those who experienced caring as a process of negotiations among family members, it led them to cooperate with other family members rather than taking on the sole responsibility of care themselves. Although those developing a partnership in the care for their family have little support in terms of personal care from other family members, like the traditionalists, care for the elderly requires them to 'become capable' - and this gives self-esteem and independence to their lives rather than stress.

However, none of these positions should be categorised as either positive or negative. Instead, an overview of the process of becoming and being a FCH will enable us to see how positive and negative factors in care for the elderly are interwoven throughout all of these types of experience.

The FCHs' position in family care is related to several factors like the way in which caregivers come to care as a licenced carer, the nature of caring tasks, whether and how family members negotiate on care, whether the caregivers co-operate in their care, the amount of support received by the caregivers, the practice of payment for care, and the relationship with the elderly as well as other family members (see Table 5.1 on the following page). 
Table 5.1 Positions in family care: Traditionalists, negotiators and partners

\begin{tabular}{|c|c|c|c|}
\hline & $\begin{array}{c}\text { Tradition } \\
\text { alists }\end{array}$ & Negotiators & Partners \\
\hline \multicolumn{4}{|l|}{ Family relations with the elderly } \\
\hline Spouse (wife/husband) & 1 & - & 6 \\
\hline Children (daughter/son) & 1 & 2 & 1 \\
\hline Children-in-law & 4 & 2 & - \\
\hline Sister-in-law & - & - & 1 \\
\hline \multicolumn{4}{|l|}{ Marital status of the caregivers } \\
\hline Single & - & - & 1 \\
\hline Married & 6 & 4 & 6 \\
\hline Bereaved & - & - & 1 \\
\hline \multicolumn{4}{|l|}{ The elderly's illness } \\
\hline Physical & 2 & 1 & 7 \\
\hline Mental & 2 & 1 & 1 \\
\hline Physical and mental & 2 & 2 & - \\
\hline \multicolumn{4}{|l|}{ An assessment of LTC needs ${ }^{91}$} \\
\hline Grade $1-2$ & - & 1 & - \\
\hline Grade 3-5 & 6 & 3 & 8 \\
\hline \multicolumn{4}{|l|}{ Usage of payments for care } \\
\hline Caregivers themselves & 2 & - & 2 \\
\hline The elderly (medical expenses) & - & 4 & - \\
\hline Both (living expenses) & 4 & - & 6 \\
\hline \multicolumn{4}{|l|}{ Support from } \\
\hline Kin (economic help, personal care) & - & 3 & 3 \\
\hline Professional (e.g. bathing services, adult day care) & 1 & - & 1 \\
\hline Kin and professional & 1 & 1 & 1 \\
\hline None & 4 & - & 3 \\
\hline \multicolumn{4}{|l|}{ Caregiver/elderly relationship during caring } \\
\hline Good & 2 & 3 & 7 \\
\hline Bad & 4 & 1 & 1 \\
\hline \multicolumn{4}{|l|}{ Caregiver/family relationship during caring } \\
\hline Supportive & 3 & 1 & 4 \\
\hline Indifferent & 3 & 1 & 4 \\
\hline Discordant & - & 2 & - \\
\hline
\end{tabular}

\footnotetext{
${ }^{91}$ The Grades of the LTCI indicates the LTC need of the elderly. An applicant to the LTC services is classified according to the LTCI grading system. Grade 1 is for the elderly with the highest needs, while Grade 5 is for those with the least. Recipients with Grade 1 and 2 are eligible to apply for both facility and in-home care services of the LTCI due to the severity of their symptoms, but those who get assessed with Grade 3, 4 , and 5 are eligible to home care services only.
} 
In two cases, I had difficulty in deciding to which types they belonged. One was the case of the eldest son who felt responsible for his mother in the sense of a moral obligation as the eldest son, but who nevertheless shared tasks with his wife in daily life. I identified the eldest son as a traditionalist because he never tried to negotiate with his siblings on care for the mother, and his wife's care for the elderly was also a part of the eldest son's obligation. The other was the case of the unmarried daughter who cared for her frail mother by herself, but whose siblings economically and emotionally supported her caring. I had difficulty in deciding whether the unmarried daughter was a 'negotiator' or a 'partner'. In the end, I identified the unmarried daughter as a partner, since her care for the mother was derived from the partnership constructed by cohabitation and an attachment over a long time instead of the traditional first son's obligation. Moreover, even if her siblings supported her, all the family thought the unmarried daughter would care for their mother and there were no negotiations on who was responsible for the mother.

Despite the differences among the three types in their positions in family care, they had several things in common. All the FCHs are licenced and paid by the public-funded longterm care (LTC) programme, but their care was family-oriented. Firstly, almost all the interviewees decided to become family care helpers due to their responsibility of care for their family, and they obtained a licence strictly to care for a family member by themselves, even if this work as care helpers made them participators in the labour market, too. Secondly, the payments for family care played only a supplementary role in caring, and the payment itself was not the main reason for caring for their family members as family care helpers. Although the interview participants obtained a licence as a care helper and were paid by the LTCI, the payments did not play a central role in their care practice. They only received a small amount of money compared to non-familial care helpers. Therefore, it was impossible to maintain the household with only this payment.

Moreover, almost all the interviewees told me that they were willing to keep on caring without the payments for care. Unlike child caregivers, who are likely to be younger and who try to keep on building their career by participating in the paid labour market, elderly caregivers in Korea were housewives or they have already retired in the case of a spouse of the frail elderly; therefore, there were fewer conflicts with regard to the balance between working in the paid labour market and caring at home. Moreover, almost all the 
interviewees had cared for children and done house chores, so they perceived their care practice for their frail elderly as part of the work they have done at home.

In the following section, I briefly summarise the characteristics of each type again.

\section{Type A: Traditionalists}

Those who complied with care 'traditions' were family caregivers who decided to become family care helpers without discussions among their family members. They took on responsibility for care in the sense of the traditional family care culture of the first son's obligation (Namgung Myoung-Hee 2010). The traditionalists faced the greatest expectations of the elderly parents among the three types. The elderly parents that the traditionalists cared for asked for caring in the sense of the first son's obligations and they tended in some cases to take the family's dedication for granted. Besides, the elderly parents had lived together with their adult children and they wanted to stay in their home where they had always lived. The experiences of the traditionalists show that the traditional care culture of the first son's obligations still lingers on in elderly care practice in Korea. Care practices of this type are related to normative guidelines to the effect that the first son is entitled to inherit his parents' property and rights. However, even if the eldest son has nothing to inherit from his father, the first son's family would still fulfil the responsibility of care for their parents in the sense of it being the 'proper thing to do' (Finch 1989)

The traditionalists experienced the most distinctive power relationship between caregivers and the elderly. Although the traditionalists complied with traditional care norms and cared for the elderly as primary caregivers, they experienced a lot of stress and conflicts in care. The stress came mainly from their previous family history when the elderly parents were in a position of power among family members compared to the caregivers.

While the traditionalists fulfilled their care responsibilities for the elderly, other family members, including co-habited family members, were less supportive of the FCHs. Instead of negotiations among family members, the traditionalists cared for the elderly alone, and not only the caregivers but also the elderly and other family members thought that the traditionalists' care for the elderly was natural and therefore was taken for granted. The traditionalists also thought themselves that the responsibility for elderly care was their calling to maintain the family, and they also felt that they should commit themselves to care in the sense of upholding a family value. Therefore, good relationships - such as 
affection between caregiver and care receiver - did not matter to the traditionalists. Rather, they were willing to sacrifice themselves because that was 'the proper things to do' (Finch 1989) in the context of family harmonisation and an ideal family. Although the traditionalists and the elderly had been in hierarchical order within the family, they were not always in conflict in their relationships. Some traditionalists complied with the normative guidelines regarding caregiving and they were in a good relationship with the elderly at the same time.

\section{Type B: Negotiators}

There were four caregivers who tried to 'negotiate' with other family members, especially their siblings, on the matter of care for their parents. The negotiators showed another type of family obligation which should be distinguished from the traditional care culture in Korea, i.e. the oldest son's obligation. Even if they were not the oldest children, they took responsibility for family care on behalf of the oldest son's family. The negotiators' attitudes towards family care were also family-centred but they differed from those of the traditionalists. The power of the elderly in decision making among the family was not as great when compared to that of the traditionalists. The elderly who were cared for by the traditionalists tended to be in a dominant position within the family. Rather, the negotiators added the elderly to their own family. For example, the negotiators let the elderly parents move into the children's house once they were in need of LTC. The negotiators tended to look for more support in terms of personal care and time from their family members compared to the traditionalists and the partners, while the partners were only economically supported by their family, who did not share other tasks regarding care for the elderly.

The payments for family care were used for the elderly instead of the caregivers. The caregivers managed the household and paid for the elderly's daily necessities, for example, groceries, medication and medical fees. The negotiators were likely to experience more conflicts with other members of their family rather than with the elderly themselves since the negotiators did not think that they were initially responsible for the care for the elderly; instead, the care responsibility was a result of the negotiations, which in some cases ended in failure. Even if the negotiators were willing to decide to become major caregivers, they experienced conflicts with other family members when the negotiators kept on caring for the elderly without (anything but financial) help from their family for many years.

\section{Type C: Partners}


The partners tended to have the closest relationship with the elderly in that they had lived together for a long time and shared their life. Almost all of the partners were spouses of the elderly, but I did not focus on the family relation itself, rather the character of the relationship between the caregiver and the care receiver. Apart from six spouses, I identified two cases of non-spousal caregivers as the type of the partners: one case (Ran) was an unmarried daughter who had lived together with her frail mother and cared for her mother, and the other (Suk)

Although both the traditionalists and the partners took most of the responsibilities for care alone, their reasons for caring for their family differed. While most traditionalists cared for their family in the sense of the first son's obligations, as a matter of 'doing the proper thing', the partners decided to become family caregivers in the sense of solidarity between individuals. The partners interacted with the elderly the most and perceived their role as a caregiver as positive, and this perception led them to be proud of being family care helpers. The LTC programme, including qualifications and licencing procedures, enabled the partners to feel that they were recognised in addition to providing economic support.

The way of sharing care responsibilities for the frail elderly depends on the position of the caregiver among the family members. According to how the family care helpers perceive and deal with their responsibility for family care, I categorised the interviewees into three types, traditionalists, negotiators and partners. Thus, it may be seen how these three types of position are related to the particular circumstances, material and attitudinal, of each carer. What the discussion of Table 5.1 cannot reveal, and what the case reconstruction that follows does reveal, is the relationships between the various factors. For example, the table cannot show the point at which help was offered or was withdrawn, or how the balance between kin and professional help shifted over time. 


\section{CHAPTER VI \\ CONCLUSION}

\subsection{Introduction: The Aim, Proposition and Scope of the research}

The thesis has sought to explore the experiences of FCHs (family care helpers) in Korea in their caregiving for their frail elderly family members. Population ageing and changes in family structures and care culture in the 2000s in Korea led to the introduction of the longterm care insurance (LTCI) for the elderly in 2008. This study began with an interest in the emerging tendency of an increase in the number of family care helpers (FCH, gajokyoyangbohosa), who are licenced family caregivers under the LTCI for the elderly in Korea.

Previous research on FCHs has mainly focused on patterns of the LTCI services provision compared to non-familial care helpers (Lee Yun-Kyung 2010; Hong Sung-Wook 2011) or on the motivations of those who have become a FCH (Yang Nan-Joo 2013; Yang Nan-Joo and Choi Inhee 2013). It has tended to pay relatively little attention to the experiences of FCHs in caring for their family members in daily life: how does the LTCI affect their daily caregiving, their relationship with their relatives who receive care and their wider family? How are the traditional care obligations changing in Korean society? Why have these caregivers become FCHs? Like other East Asian countries, Korea has been known as a country where the family-centred care culture based on kin relationships has been regarded as 'the proper thing to do' (Finch 1989; Finch and Mason 1991) in the sense of the first son's obligations, whose character has been subject to very little empirical investigation in recent years following the implementation of the LTCI. Against this background, understanding the lived experience of the FCH is important in that they are situated on the boundary between formal and informal care.

Following the empirical Chapter V, which made use of a theoretical framework based on care responsibility in discussing the research findings, this concluding Chapter VI will revisit the main findings and discuss the implications of the empirical findings and also discuss the contributions and limitations of the study, as well as making suggestions for future research. Section 6.2 summarises the contents of each chapter, including the context 
of the study (Chapter II), the development of the research questions and arguments (Chapter III), the methods of data collection and the analysis of the interviews to answer the research questions (Chapter IV), and the empirical findings (Chapter V). This chapter (VI) will also include reflections on the relevance and value of the concept of care responsibility for Korean society in Sections 6.3 and 6.4. Section 6.3 discusses the implications of the experience of the FCHs at a macro-level. Section 6.4 presents the implications of the experience of the FCHs at a micro-level. The chapter ends with some concluding remarks on the experiences of the FCHs, the limitations of this study, and further research suggestions.

\subsection{Summary of the Thesis}

Throughout the thesis, the main focal point of the analysis has been the responsibility for elderly care in Korea. In Chapter I, I introduced the background and objectives of the research. This study aimed to investigate the experience of the FCHs and to contribute to revealing how the responsibility for elderly care is shared at two different levels, at a macroand a micro-level. At the macro-level, the FCHs play two different roles as long-term care service providers, as licenced caregivers and as LTCI recipients at the same time in the service delivery system in the LTCI. Due to the overlapping status of the FCHs in the LTCI, investigating the experiences and perceptions of the FCHs regarding their social status and social rights in caring for their family members has enabled us to look from a different angle at the social care arrangements in Korea. At the micro-level, FCHs are the major caregivers for the elderly among family members, and the everyday experiences of the FCHs on becoming and being a FCH have enabled us to see how family practices of elderly care vary when they are negotiated as to the manner of care for the elderly. Therefore, the experiences of the FCHs on becoming and being a licenced caregiver have enabled us to understand these issues both at a macro- and a micro-level. Focusing on an increase in numbers of the FCHs after the implementation of the LTCI, I set out three research Questions:

1. What is the meaning of caregiving as a FCH?

2. How do the FCHs construct their position as users and service providers of the LTCI?

3. How do the FCHs take the care responsibility for their old family members?

Before investigations on the experiences of the FCHs, I formulated initial assumptions as follows: the social status of the FCHs would be somewhere between workers and family 
members; through the introduction of the LTCI programme, the FCHs would concerns about their social rights as individual workers- for example, their entitlement to social insurance benefits.

Then, in Chapter II, I described the context of the research by explaining life in old age and family relations in Korea. Old age is the age when people confront various challenges, the weakening of their economic power and their physical ability, as well as changes in family relations. The traditional forms of intergenerational financial and personal care support in Korea have been changing in a way that has led to their decrease. This explanation enables us to understand family life in Korea. An overview was given of the Korean background and the relevant cultural aspects of later life and family relations. This is important since elderly care policy and practices have been significantly affected by the traditional care culture. This chapter helped us to understand the cultural context that led to family care helpers who are licenced family caregivers, as well as justifying the choice of the country context.

Specifically, Chapter II showed how care responsibilities for elderly care are shared between the government and the family in Korea by explaining the trajectories of recognition hours for the LTCI services offered by FCHs. The FCH programme in the LTCI can be understood as following a trajectory of the compromises regarding the responsibilities for elderly care between state and family within the long-term cultural legacy of Korean family-centred values. In these compromises, it became more difficult for FCHs to obtain social rights to pension benefits and individual entitlements as economic participants. The Korean case of the FCH programme in the LTCI helps to set out criteria for the law- and policy-making in the light of the FCH labour conditions, both as family members and service providers of the LTCI.

Chapter III reviewed various concepts and theories to establish an analytical framework. The literature review sought to provide an overview of the literature on social rights and social care in general and the elderly care arrangements in the Korean welfare state and family obligations and responsibilities in the sense of the Korean care culture in particular. Based on the literature review, Chapter III outlined the theoretical framework used to conceptualise care responsibilities. Gaps in the literature were identified, including the lack of any studies of the lived experiences of FCHs who have a status somewhere between family members and workers. 
Based on these assumptions, this study has investigated the following questions: How did informal caregivers decide to become FCHs? What is the lived experience of the FCHs in both public and domestic areas? How do they experience it in terms of their social status and their rights? How do they manage the conflicts, if any, stemming from their overlapping roles in the family as care-providing workers and as family members? In answering these questions, the study interviewed 18 FCHs who had cared for or were caring for infirm family members at home.

Chapter IV explained the procedures of the method that I adopted in this study. A qualitative empirical method was applied to investigate the lived experience of the FCHs. Chapter IV focused on the methodology and justified the choices made in terms of the problem-centred interviews (PCIs) as research methods, and the use of biographical reconstruction as an approach to interpretative analyses. To recruit the interviewees, I made field trips in 2015 and 2017 and conducted 18 PCI interviews with family caregivers who had cared for or were caring for frail senior family members as a licenced care helper. For analysing the collected data, I followed the method of case reconstruction as suggested by Rosenthal (1995; 2011; 2018). This analysis comprises six steps, including analysis of the biographical data, thematic field analysis, reconstruction of the experienced life history microanalysis of individual text segments, contrastive comparison of life history and life story, and development of types and a contrastive comparison of several cases. These analytical steps were utilized to provide a better understanding of the experiences of FCHs.

Chapter V presented empirical findings of the experiences of family care helpers. After analysing all the cases, there emerged several themes and hints of how to achieve a comprehensive understanding of the experiences of the FCHs. Almost all the interviewees mainly stated the relationship with the elderly based on their family history and their emotions in caring for the elderly. As a family practice (Morgan 1996; 1999), the interviewees mentioned their complicated feelings in caring for their family. As a relationship, family care practices are affected by the former family history and the caregivers' biography, including their backgrounds, their careers and their relationships with the elderly and with other family members. There were some noticeable emotional responses which emerged in the interpretation of the interviews.

- The feeling of guilt

- A justification for accepting/rejecting care for the elderly 
- Being proud of becoming a FCH

\section{The feeling of guilt}

Some of the first-daughters-in-law expressed their feelings of guilt in caring for infirm family members during the interviews. The first daughters-in-law in Korea had been major caregivers in caring for their infirm parents in the sense of the first son's obligations, in which the first son's family has taken responsibility for maintaining an extended family. In a caring relationship between the elderly and the daughters-in-law, the elderly were usually in a dominant position as seniors to be respected within the family, but they needed the daughter-in-law's help in the activities of daily living. In the course of this, both the elderly and the daughters-in-law experienced a discordance between the order in the family and the capacity for independent living.

From the viewpoint of the daughters-in-law, they experienced ambivalent feelings between emotional stress and internal moral guidelines requiring them to respect the elderly. Even though the daughters-in-law suffered from conflicts with the elderly in their everyday life due to the parents' authoritative attitude towards the daughters-in-law, they were still willing to keep on caring for their frail parents as the 'proper thing to do' (Finch 1989). The sense of guilt of the daughters-in-law could be interpreted as a coping strategy to handle the internal contradictory feelings they felt in helping the authoritative parents who were not very cooperative even in caring for themselves.

The previous studies on daughters-in-law's care for the elderly used to focus on the care burdens and the stress involved in caring (Kim Hyunjoo 2001, Seo In-Soon 2003) but there was little mention of complicated feelings like guilt. This empirical finding of the ambivalent feelings of the daughters-in-law shows another approach to the understanding of the complicated relational nature of family caring in Korea and similar countries, where a family-centred care culture is dominant.

\section{A justification for accepting/rejecting care for the elderly}

Several adult children mentioned that they experienced challenges and difficult situations in caring for the elderly which came not from everyday life in caring but from the process of decision making and the negotiations with other siblings on the way of caring for the elderly. Given the traditional family-centred care culture in Korea, the first son's family used to live together with their old parents and care for them until the end. However, as the 
family structure and adult children's attitudes towards elderly care have changed, the normative guidelines for elderly parents' care have also become blurred. The first son's obligation still remains in place somehow, but other children, including other sons and daughters, have started to take responsibility for care for their infirm parents. The conflicts among siblings in decision making on the care for their parents showed that elderly care in Korea is not just a practice between two individuals but a family practice among all the family members.

In the course of the decision making on caring for the elderly, the daughters-in-law who were not the first daughters-in-law or the daughters needed clearer justifications for taking responsibility for the elderly. The justifications related not only to their wills and circumstances but also to why other siblings did not take care responsibility for their parents. Sometimes, these non-traditional family caregivers in the context of the Korean traditional care culture expressed their sense of hurt caused by the other siblings, especially the first son's neglecting of his duty for his frail parents. At the same time, the daughters and younger daughters-in-law tried to figure out the justification for their siblings' unsupportive attitudes. This empirical finding in this study is in line with the argument of Finch and Mason (1993) that filial piety, which is an important element in familialism, is completed through one's practical behaviour and considerations of moral reasoning about what is 'the proper thing to do'. This empirical finding shows how the social meaning of caring for infirm parents has been newly constructed though legitimating excuses used for justification to avoid caring for the elderly. When one wants to avoid family obligations, then one needs to justify one's rejection. However, the experiences of the daughters in Korea shows that not only the persons who reject family obligations but also the persons who take on the responsibility need to justify the rejection of the other siblings to care for the elderly in order to accept the task that they take on.

\section{Being proud of becoming a FCH}

All of the old-aged wives interviewed in this study mentioned that they were the one and only primary caregiver for their husbands. In addition, being a licenced caregiver (FCH) was more important for old-aged wives compared to adult children caregivers. As the more adult children live separately from their old parents, the support for economic and personal care has lessened, thus the elderly households need measures for independent living by themselves. As the national pension system (NPS) was first implemented in late 1980, most 
of the present elderly receive no old-age income from old-age pension benefits from the NPS but make do with a patchwork of income sources which include public transfers like basic pension benefits, private transfers from their adult children and labour income from part-time jobs ${ }^{92}$. Given that it is important for the present elderly households to secure their old-age income, the payments for care for old households are among the major income sources.

Besides, the old-aged wives never assumed that they could rely on their adult children for personal care. This shows that normative guidelines for elderly care in the sense of the first son's obligations have diminished not only in the minds of adult children who give care but also in old parents' own perceptions of elderly care. In this sense, the old-aged caregivers focused on the symbolic meaning of obtaining a licence as a care helper. When the caregivers told the reason why they decided to become caregivers they said that caring at home provided by family caregivers was much better than ageing in a care facility. Also, the old-aged wives expressed their sense of obtaining qualifications as licenced care helpers as an achievement and they felt proud of their care for their spouses even though they were too old to work in the paid labour market. With regard to the family care culture, the interviewees who were daughters and daughters-in-law did not expect their children's support in personal care, even though they followed the family's obligations in providing care for their parents.

\section{Three types of the FCH: the traditionalists, the negotiators, and the partners}

Based on the emotional responses of the interviewees and their ways of taking on care responsibilities, I differentiate between three broad sets of family care helpers' positions in their negotiations with the other family members on elderly care. The FCH's position in family care was related to the way in which caregivers come to care as licenced caregivers; the amount of support from other family members and outside the home; the receiving of payments for care; and the relationship with the elderly as well as with other family members. In the end, I arrived at three ways of taking care responsibility for the elderly: traditionalists, negotiators and partners.

\footnotetext{
92 See more details in Chapter II
} 
The traditionalists were expected to follow caring traditions and to be mainly responsible for the care of the elderly in their families. Among the three types of caregivers, the traditionalists were under the most explicit power relations, in which the elderly members were in a dominant position in caring relationships. Not all the traditionalist conflicted with the elderly, and there were caregivers who complied with the family obligations and had good relationships with the elderly and with other family members. However, the traditionalists who were not in good relationships with the elderly had a heavy burden with mixed ambivalent feelings. In general, the frail elderly were known to be in a dependent position, requiring caregivers for activities in their daily life (ADL), but the relationship between traditionalists and the elderly in Korea was different from the traditional relationship between the dependent and the independent. This result is consistent with Freed (1990)'s study on the experiences of Japanese daughters-in-law who cared their mothers-in-law in the sense of moral obligations, which showed that the daughters-in-law felt mixed ambivalence in caring for their mothers-in-law when the mothers had objected to the marriage in the past. The traditionalists received neither economic support from close and extended kin nor personal care assistance from cohabiting family members. Also, the traditionalists perceived elderly care as their own duty, but they suffered from the indifference of the other family members. In this sense, the traditionalists viewed the payments for care as compensation for their care labour.

The negotiators tried to negotiate with other family members on caring for the elderly. For the negotiators, taking responsibility for care for the elderly was a result of the negotiations among other family members. Daughters and younger daughters-in-law were identified as the negotiators. Although they were not part of the oldest son's family, they took on responsibilities for family care on behalf of the oldest son's family. In this sense, the negotiators needed to provide justifications for a substitution for the oldest son who was traditionally supposed to take on the family's duty. In contrast to the traditionalists, the negotiators were in the dominant position in decision making on elderly care among the family members and were likely to receive support financial support from their extended kin. However, when the negotiations and justifications did not work in the decision-making on elderly care, the negotiators were likely to experience more conflicts with their siblings since they were initially responsible for the care for the parents. The parents have little power in the negotiations over the way of care regarding the person, the location and the resources. In this sense, the payments were used for the elderly's living expenses. 
Lastly, the partners tended to have the closest relationship with the elderly in that they had lived together for a long time and shared their life. Almost all of the partners were spouses of the elderly, but I did not focus on the family relation itself, but on the character of the relationship between the caregiver and the care receiver. Although both the traditionalists and the partners took on most of the responsibilities for care alone, their reasons for caring for their family differed. While most traditionalists cared for their family in the sense of the first son's obligations, as a matter of 'doing the proper thing', the partners decided to become family caregivers in the sense of solidarity between individuals. The partners interacted with the elderly the most and perceived their role as a caregiver as positive, and this perception led them to be proud of being family care helpers. The LTCI programme, including its qualifications and licencing procedures, enabled the partners to feel that they were recognised in addition to providing economic support.

Through the analysis of the interviews I reached a provisional conclusion that although the FCHs differed in coping with the care for the frail family members, they decided to become a FCH in the sense of their family obligations instead of being an individual care worker. With regard to social rights, most of the interviewees perceived themselves as family caregivers, and they told me that although they decided to become licenced caregivers because of the payments for care, they were willing to keep on caring without the payments. Moreover, they did not care about whether they were entitled to pension benefits, unemployment insurance and occupational benefits as an individual worker. Given that most of the caregivers were in their mid-fifties and over and had stayed at home as housewives, there were fewer conflicts with regard to the balance between working in the paid labour market and caring for the elderly at home.

In the analysis of the experiences of the FCHs, I also discovered the peculiar relationships between the FCHs and the care centres in the service delivery of the LTCI. The FCHs were less under the control of the care centres, in which they made contracts for payments for LTCI services. In the service delivery system of the LTCI, there were no differences between non-familial care helpers and family care helpers regarding the qualification process and service delivery. The FCHs completed courses and obtained licences as care helpers. In addition, they made a contract with a care centre, which gave supervision and reported the LTCI services offered by the FCH to the LTCI. However, the care centres neither controlled nor supported the family caregivers. The centres only reported and 
managed records on service hours offered by the FCHs. This result on the relationship between the FCHs and the care centres supports the idea that the FCHs have more characteristics of informal family caregivers than of care workers in the paid care market.

The empirical findings suggest the opposite conclusions from those that I expected in Chapter I and III regarding the perceptions of the FCHs on their social status and family responsibilities. In the next two sections, Sections 6.3 and 6.4, I will discuss the theoretical implications of the empirical findings. Whilst Section 6.3 will put the empirical findings into the discussion regarding the arguments on social rights and social care and, at the macro-level, Section 6.4 will reflect on the concept of care responsibility and the care culture for the elderly among family members.

\subsection{The Family's Responsibility for Elderly Care in Social Care}

As was mentioned in section 6.2, the FCHs considered their caregiving for the old family members totally as family practice based on family obligations, as the payments for family caregiving were not enough for them to be entitled to the national pension system (NPS) benefits as an individual worker. The FCHs rather prepared for their old-age income in different ways, e.g. the adult children of this study paid the contributions for the NPS as a voluntarily insured person, or else the wives of this study were already of retirement age, so they were supported by their adult children.

In this section, I consider the implications for the findings of this study by answering questions posed in Chapter III: Who are the FCHs in terms of their social status? When formal elderly care is provided, how does informal family care change? How can their social rights be insured?

\section{Who are the FCHs in terms of their social status?}

According to the prescribed regulations on the qualifications and payments for care in the LTCI, there were no differences between family care helpers and non-familial care helpers except for the limited recognition of the hours for the LTCI services offered by the FCHs ${ }^{93}$. However, the limited recognition of the FCHs' caregiving would lead to a failure to expand the extent of the social rights of the FCHs who care for their infirm elderly relatives at

\footnotetext{
${ }^{93}$ See more details in Chapter II
} 
home. This means that there could be a gap between the data and the factors at a collective level and the perceived status of the persons directly concerned. To solve this puzzle, the study identified whom the FCHs perceived themselves as being regarding their social rights based on their lived experiences and their needs in caring for their family members.

After the implementation of the LTCI in Korea, the social status of the FCHs did not change in terms of social rights in the objective sense regarding pension benefits, unemployment benefits and occupational accident compensations because of the limited recognition of the LTCI services offered by the FCHs. However, it is more notable that the FCHs did not consider their social status and social rights as individual workers since they perceived themselves as family caregivers. The daily life of the FCHs shows why they thought of themselves as family caregivers in that they continued their previous relationships and care patterns at home after obtaining their qualifications as care helpers; and they regarded themselves as users instead of service providers in their relationship with care centres, who exercised little supervision and control over the FCHs compared to the non-familial care helpers. Through the investigation of the lived experience of the FCHs, this study contributes to determining how the FCHs regard themselves, as falling somewhere between family caregivers and care workers. In addition, this study has shown that the implementation of the social service programme may not always lead to the expansion of the social rights of the person concerned.

\section{Supported familialism instead of defamilialisation through the market}

Although the LTCI in Korea did not change the social status of the FCHs regarding their social rights as individual workers, the payments for LTCI services offered by the FCHs can be understood in various ways. As Saraceno (2016) argues, the meaning of the payments for care varies in their different forms between support for familialism and for defamilialisation (Saraceno 2016: 322). She used the Organisation of Economic and Cooperation and Development (OECD)'s data at a collective level to identify the forms of familialism/defamilialisation produced by the LTCI in Korea, and she concluded that Korea was 'moving in the direction of a greater (via compulsory insurance or state subsidies) support for defamilialisation through the market' (ibid. 324).

However, according to the empirical findings of this study, we should be doubtful about the defamilialisation of elderly care after the implementation of the LTCI. Rather, Korea is moving from familialism by default towards supported familialism in its elderly care 
policy. When they obtain a licence as care helpers, the family caregivers are compensated for their care for the infirm elderly, which means that the payments for care are designed to partly compensate the family caregiver (supported familialism) instead of paying for a hired carer (defamilialisation). This misconception of the degree of defamilialisation produced by the LTCI is due to overlooking the existence of the FCHs who are hidden within the market-driven elderly care programme, which allows family caregivers to become 'paid' caregivers within the market. This strategy of the Korean government is in line with Kodate and Timonen (2017)'s argument that the welfare states encourage family care inputs for the long-term sustainability of the care regime in elderly care. Through policy changes, the government is expanding the role of the family both explicitly and implicitly 'through the back door' (Kodate and Timonen 2017, 293). The empirical findings on where the FCHs fit into the LTCI services delivery, as both providers and users at the same time, contribute to the discussion by allowing the FCHs to become visible.

\section{Did the family's responsibility for elderly care decrease after the implementation of the LTCI?}

Based on the findings and arguments on the experiences and perceptions of the FCHs, it is doubtful whether the family's responsibility for elderly care decreased after the implementation of the LTCI. Although the FCHs' care is compensated by the LTCI's, the family caregivers take on different kinds of responsibility for elderly care. There is no option between receiving cash or services in the LTCI and the caregivers deciding to become licenced caregivers, who follow qualifications and regulations such as reporting their care service through a device such as a radio frequency identification (RFID) like a care helper in the paid care market. While the care centres do not control the FCHs in caring for the elderly, the government's policy on the management system for the care offered by the FCHs has an impact on the expansion of the infrastructure of the LTCI in the sense of the creation of jobs. This is in line with the arguments of Wood and Gough (2006) that Korea can be identified as a productivist welfare state where social policies are subjected to economic and development policies.

\section{The family caregivers as active and rational subjects}

Throughout their accounts of the decision to become a FCH, the interviewees in this study showed themselves to be active and rational individuals who are coping with the ongoing changes in elderly care policy and care culture. I interpret becoming a FCH as a choice of 
families to share their care responsibility with the Korean welfare state while fulfilling their family obligations at the same time. This finding can provide evidence for arguments that the payments functions as care allowance for family care in reality (Lee Hyunsook 2011; Lee Min-Sook 2012; Lee Yun-Kyung 2010; Yang Nan-Joo and Choi In-Hee 2013).

Previous studies have tended to evaluate family caregivers as stubborn and vulnerable people with care burdens (Lee Yun-Kyung 2011; Hong Sung-Wook 2011). What is worse, they are described as a threat to the fiscal stability of the LTCI. However, the FCHs actively decided to become licenced caregivers not only for the financial gains but also for considerations of moral and relational status and for the functional advantage of their elderly relatives. This study shows the existence of different types of caregivers in Korea which has been known for its traditional elderly care culture as the first son's obligation.

\subsection{Care Responsibility as a Family Practice}

At a micro-level, I consider the implications by answering questions in relation to family care practice: Given the social changes and the implementation of the LTCI, how have families' perceptions of the ideal of elderly care changed? How do family members make decisions on elderly care after the implementation of the LTCI? What challenges remain for elderly care in Korea?

\section{Has the ideal of elderly care changed?}

The elderly care practices in Korea still come from the notion of care responsibility in the sense of the family's obligations. However, the experience of care for frail elderly family members depends on the caregivers' perceptions of who among family members should take on the care responsibility for the elderly. The findings on the negotiations over who takes responsibility for care for their infirm elderly relatives show that family obligations work at a normative level, but that it is not easy to reach a consensus on what the 'proper thing to do' is.

As mentioned in the previous chapters, the family-centred elderly care culture in Korea has affected the ideal of elderly care, which is to be cared at home by family members in the sense of the first son's obligation. Findings of this study showed that the ideal of elderly care still remains on a normative level, although in practice the major caregiving responsibilities are being increasingly transferred in parts from the wife of the oldest son towards other family members like daughters and wives of the elderly. Nevertheless, the 
ideal of elderly care changed in that the family member chooses actively to become a FCH, and this can be interpreted as the family combining the formal supports with their informal care based on the family obligations. When it comes to the ideal of elderly care, the family do not have to choose between the formal and the informal, they rather combine the two settings by transforming themselves to a care provider in the LTCI. In other words, the care responsibility for the elderly could transfer from the family to the state not only by choosing between the two settings but also by transforming the characteristics of the caregivers.

The finding of this study implies that the ideal of elderly care in Korea is changing regarding the cost share, after the implementation of the LTCI, adapting the idea that combining two settings is better than caregiving only in the informal setting. This finding is in line with Yang Nan-Joo and Choi In-Hee (2013)'s findings that family caregivers' coping strategies with the elderly care vary depending on the family's need and the LTCI services the family can select.

Besides, the existence of a variety of family caregivers is a result of the ongoing changes in the family structures and family care culture in Korea. As a result of rapid urbanisation and industrialisation, the present generation who are carers for the present generation of elderly people does not expect that they will receive personal care from their adult children. The changes in life in old age due to the trends of decreasing cohabitation with the first son's family and an increasing need for independent living of the elderly parents make payments for care a significant source of income in old age. The interaction of family obligations in the form of the traditional care culture and the cash for care system under the LTCI can generate forms of sharing care responsibilities that differ from the social care in Western countries that are mainly based on individualistic social rights.

\section{How do family members make a decision on the form of elderly care after the implementation of the LTCI?}

While the ideal of elderly care regarding who cares at a collective level has changed in part, the decision of family members on who cares at family level did not change after the implementation of the LTCI. According to the findings of this study, most of the FCHs of this study decided to become a FCH after they were assigned as a major caregiver. Two different but linked decisions on becoming the caregiver can be interpreted as the two contexts. On the one hand, becoming a major caregiver is first determined by the family's expectations based on cultural values and social norms. Due to such factors, it is hard for 
the family caregivers to avoid the responsibility of elderly care assigned to them by the family despite the offers of elderly care provided outside the home. On the other hand, becoming a FCH after the decision of becoming the major caregiving among family members could be considered as one strategy of coping with the challenges after becoming a family caregiver. In this regard, the payments for the FCH function as a support of the family caregiver in caring for the elderly at home, but the payments themselves cannot affect a decision among family members on who is supposed to be the major family caregiver.

\section{Which challenges remain?}

Although the FCH programme in the LTCI provides the family caregivers with the possibility of sharing the care responsibility for the elderly, the FCHs experienced challenges in caring for their family members. The typology of the FCHs of this study enables to reveal the varieties of the experiences of the family caregivers ${ }^{94}$. This finding provides a necessity for the customized supports depending on the caregiver's needs. For instance, Jina's experience ${ }^{95}$ of becoming a FCH shows that although many FCHs who offer in-home care services are family care helpers, there is neither a customised programme for family caregivers nor support for caregivers who need sitting for their elderly. Most family care helpers care only for their family member (Hong Sung-Wook 2011; Lee Yun-Kyung 2010); it must then be enough for the FCHs to get the specific knowledge they need regarding the illness or problems of their family members. From the perspective of the family carers, they spend time and money unnecessarily.

The Korean case of the FCH programme in the LTCI helps us to set out criteria for policy making in the light of the FCHs' experience in daily life. Moreover, the case of FCHs in Korea has implications for other Asian countries which seek to solve the problem of constructing public elderly care in a situation where traditional care ideals still linger while the state at the same time wants to ease the financial burden of elderly care.

\footnotetext{
${ }^{94}$ See details in Section 5.5 or in Section 6.2 for a summary.

${ }^{95}$ See details in Section 5.4 or in Section 6.2 for a summary.
} 


\subsection{Limitations, and Suggestions on Further Research}

This study also has several limitations and it offers further research suggestions.

With regards to the methods, although this study tried to analyse the interview according to the suggestions of the biographical research, it was difficult to generate descriptions on the self-presentation of interviewees. The self-presentation could be generated from an open and general question without an interruption of the interviewer. However, I could not follow the suggestions since I had not fixed the analysis strategies when I conducted the interviews on the field trips. Specifically, my initial opening question has mainly focused the illness of the elderly and how family caregivers decide to become a FCH, so they were likely, to begin with, a specific excerpt regarding the illness instead of their life story as a whole.

Although most of the interviewees in this study were women, this study has paid little attention to gendered caregiving practices. Rather, this study has touched in part on inequality of educational opportunity between boys and girls in their childhood due to the patriarchal order in the distribution of a family's resources. Complicated characteristics of care have become one of the main subjects of care policies. Kremer (2007) argues that it would be hard to understand the patterns of women's labour without any observations through the care lens and that it should be reconstituted as a right to participate not only in the paid labour market but also in care outside the paid labour market.

Although this study concerns the experiences of FCHs in negotiations on the elderly care, the family context, including power relations and relationships among family, the process of becoming and being a FCH, was only shown from the perspective of the $\mathrm{FCH}$, the major caregiver of the elderly. Therefore, I suggest further research on the phenomenon focusing on family dynamics and the process of the decision making on caring for the elderly by conducting interviews with different sides of family members individually or in groups.

Lastly, this study put great emphasis on the family-centred care culture in Korea and argued that this culture led to the implementation of the FCH programme of the LTCI. However, almost all the cases in this study were of people who live in cities, and this is not enough to enunciate clearly the influences of care culture and values on the care service arrangements of a welfare state. Further comparative studies of convergences and 
deviations in care arrangements among areas in different care cultures and values should be carried out.

At a macro level, this study shows that the FCH programme of the LTCI is interwoven with other social insurances - the income security programmes like the national pension system (NPS) and the basic pension (BP), the employment insurance, and the occupational accident insurance in Korea. However, this study has touched little on how the institutions themselves are intertwined with each other in the question of caring for the elderly as a FCH. This could be a question for future research. 


\section{References}

An, Mi-Young. 2017: "Welfare States and Care Arrangements: Care time mix approach and its application to Japan and Korea”, Social Policy and Society, 16(2): 183-198.

An, Mi-Young. and Ito Peng. 2015: "Diverging Paths? A comparative look at childcare policies in Japan, South Korea and Taiwan”, Social Policy and Administration, 50(5): 540-558.

Anttonen, Anneli., John Baldock., and Jorma Sipilä. 2003: The Young, the Old and the State. Social Care Systems in Five Industrial Nations, Cheltenham: Edward Elgar.

Arksey, Hilay. and Caroline Glendinning. 2007: “Gender Differences in Informal Caring”, In Allen, G. (ed.), The Sociology of the Family: A Review, Oxford: Blackwell: 312-339.

Ashworth, Georgina. 1999: “The Silencing of Women”, In Tim Dunne and Nicholas J. Wheeler (eds.), Human Rights in Global Politics, New York: Cambridge University Press.

Beteta, Hanny-Cueva. 2006: “What is Missing in Measures of Women's Empowerment?”, Journal of Human Development, 7(2): 221-241.

Bien, Barbara., Beata Wojszel., and Elzbieta Sikorska-Simmons. 2007: "Rural and Urban Caregivers for Older Adults in Poland: Perceptions of positive and negative impact of caregiving”, The International Journal of Aging and Human Development, 65(3): 185-202.

Bradley, Karen. and Diana Khor. 1993: "Toward an Integration of Theory and Research on the Status of Women.”, Gender \& Society, 7(3): 347-378.

Brewer, Loretta. 2000: “Gender Socialization and the Cultural Construction of Elder Caregivers”, Journal of Aging Studies, 15(3): 217-235.

Bryman, Alan. 1992: "Quantitative and Qualitative Research: Further reflections on their integration”, In Julia. Brannen(ed.), Mixing Methods: Qualitative and Quantitative Research, 57(7), Aldershot, UK: Ashgate.

Byun, Soojoo. and Jae-Eun Seok. 2010: "Quality Assurance Strategy for Long-Term Care Workforce”, in International Symposium on Performance-oriented Development Measures for the Long-Term Care Insurance for the Elderly, Seoul: Ministry of Health and Welfare.:193-219. (In Korean)

Chang, Ji-Yeon., Gacheong Bu., Hyejeong Lee., Hyungu Shin., Cheolhee Lee., Sukrang Chang., Sungil Cho., and Lisa F. Berkman. 2008: Comparative Study on Labour Market and Employment of the Middle-Aged, Working Paper 2008-03, Seoul: Korea Labour Institute (KLI): 1-165. (In Korean)

Chang, Kyung-Sup., Meejung Chin., Miai Sung., and Jaerim Lee. 2015: “Institutionalized Familialism in South Korean Society: Focusing on income security, education, and care”, Family and Culture, 27(3): 1-38. (In Korean)

Cantor, Marjorie H. 1979: "Neighbours and Friends: An overlooked resource in the informal support system”, Research on Aging, 1(4): 434-463.

Chae, Wan. 2004: “A Study on Brand Naming of Apartments”, Korean Semantics 14, 2004.6: 129. (In Korean)

Chang, Byoung-Won. 2005: “Yoyang or Subal, for the Elderly?”, news articles, Daily Jungang, http://news.joins.com/article/1728441(access on October 1 $1^{\text {st. }}$ 2015, In Korean) 
Chang, Jun-Dong., Je-Hyun Yoo., Sang-Soso Lee., Tae-Young Kim., Kyu-Hak Jung., and YongKuk Kim. 2010: "Bipolar Hemiarthroplasty for Hip Fractures in Patients Aged over 90 Years: The factors influencing the postoperative mortality”, Journal of Korean Hip Society, 22(4): 283-290. (In Korean)

Change, Kyung-Sup., Meejung Chin., Miai Sung., and Jaerim Lee. 2015: “Institutionalized Familialism in South Korean Society: Focusing on income security, education, and care”, Family and Culture, 27(3): 1-38. (In Korean)

Cheon, Byung You. 2010: "Problems of Long-Term Care Worker for the Elderly and Search for Alternative Model and Policy Improvements in Korea”, Korea Social Policy Review, 17(3): 67-91. (In Korean)

Cho, Cheung-Moon. 1997: “The Study of the Bilateralization of Korean Kin Relationship”, Journal of Korean Women's Studies, 13(1): 87-114. (In Korean)

Cho, Hye-Young., Seong-Eun Boo., and Jong-Hwa Jeong. 2015: “A Qualitative Study on the Care Experience for Parents-in-law with Dementia”, Korean Review of Crisis \& Emergency Management, 11(9): 135-164. (In Korean)

Cho, Myoung-Ok. 1999: “An Ethnography of Succession of Caring Behaviors for the Elderly”, Journal of Korean Gerontological Nursing, 1(1): 42-71. (In Korean)

Choi, In-Hee. 2012: “Old Women’s Caregiving for Their Spouse: How to mitigate their care burdens?”, Issue Paper 2012-3, Korean Women Development Institute (KWDI). (In Korean)

Choi, In-Hee., Eun-Ji Kim., Soo-Yeon Chung., and Nan-Joo Yang. 2011: “The Impact of the Korean Long-Term Care Insurance System on the Beneficiaries and Their Family Caregivers: Focusing on quality of life and family relationships”, Research Paper No.1, Korean Women Development Institute (KWDI). (In Korean)

Choi, In-Hee. and Jung-Hyun Kim. 2013: “Characteristics of Certified Family Care Providers(gajokyoyangbohosa) and Informal Caregivers, and Predictors of Their Caregiving Burden”, Family and Culture, 25(3): 159-185. (In Korean)

Choi, In-Hee., Young-Ran Kim., and Jihye Yeom. 2012: The Profile of Family Caregiving as Provided by Female Older Adults in Korea, Korean Women Development Institute. (In Korean)

Choi, Sung-Ho. 2008: “A Study on the Systematic Building of Care Worker Infrastructure: Instruction in Korea by German cases”, Zeitschrift der Koreanisch-Deutschen Gesellschaft für Sozialwissenschaften, Summer: 129-160. (In Korean)

Choi, Won-Seok. and Jin-Sook Lee. 2014: “A Study on Factors Influencing for Work Plan of Female Baby-Boomer”, Health and Social Welfare Review, 34(1): 299-327. (In Korean)

Chon, Yongho. 2015: “An Exploratory Qualitative Study on Relationships Between Older People and Home Care Workers in South Korea: The view from family carers and service providers”, Ageing and Society, 35(3): 629-652.

Clyburn, Leah D., Michael J. Stones., Thomas Hadjistavropoulos., and Holly Tuokko. 2000: "Predicting Caregiver Burden and Depression in Alzheimer's Disease”, Journal of Gerontology, 55B(l): 2-13. 
Colombo, F. et al. 2011: Help Wanted? Providing and Paying for Long-Term Care, OECD Health Policy Studies, OECD Publishing.http://dx.doi.org/10.1787/9789264097759-en

Chu, Yousun. 2011: “Institutional Improvement of Working Conditions of Care Workers with Emphasis on Care Assistants”, Labor Studies, 21: 67-108. (In Korean)

2013: "Institutional Improvement of Working Conditions of Care Workers with Emphasis on Care Assistants”, Labour Studies in Labor Education and Research Institute, Korea University, 21: 67-108. (In Korean)

Chung, Eun-Hee. 2011: Analysis on Behavior and Financing for the Family Caregiver's Service Delivery in Korea: Focusing on the Revision of System in August, Chungang University Master dissertation. (In Korean)

Daly, Mary. and Jane Lewis. 1998: "Introduction: Conceptualising social care in the context of welfare state restructuring”, in: Lewis, Jane. (ed.) Gender, Social Care and Welfare State Restructuring in Europe, Aldershot: Ashgate: 86-103.

2000: "The Concept of Social Care and the Analysis of Contemporary Welfare States", British Journal of Sociology, 51(2): 281-298.

Dwyer, Jeffrey W. and Michael K. Miller. 1990: "Differences in Characteristics of the Caregiving Network by Area of Residence: Implications for primary caregiver stress and burden”, Family Relations, 27-37.

Dwyer, Jeffrey W. and Karen Seccombe. 1991: "Elder Care as Family Labor: The influence of gender and family position”, Journal of Family Issues, 12: 229-47.

Dwyer, Peter. 2000: Welfare Rights and Responsibilities: Contesting Social Citizenship, Bristol: Policy Press.

Eichler, Melanie. and Birgit Pfau-Effinger. 2009: “The 'Consumer Principle' in the Care of Elderly People: Free choice and actual choice in the German welfare state”, Social Policy and Administration, 43(6): 617-633.

Engster, Daniel. 2007: The Heart of Justice: Care Ethics and Political Theory, Oxford: Oxford University Press.

Esping-Andersen, Gøsta. 1990: The Three Worlds of Welfare Capitalism, Princeton, N.J: Princeton University Press. 1999: Social Foundations of Postindustrial Economies, Oxford: Oxford University Press.

2009: The Incomplete Revolution. Adapting to Women's New Roles, Cambridge; Malden: Polity.

Estévez-Abe, Margarita. and Yeong-Soon Kim. 2014: "Residents, Prime Ministers and Politics of Care: Why Korea expanded childcare much more than Japan”, Social Policy \& Administration, 48(6): 666-685.

Evers, Adalbert. and Ivan Svetlik. 1993: Balancing Pluralism: New Welfare Mixes in Care for the Elderly, Aldershot: Avebury.

Freed, Anne O. 1990: “How Japanese Families Cope with Fragile Elderly”, Journal of Gerontological Social Work, 15(1/2): 39-54.

Finch, Janet. 1989: Family Obligations and Social Change, London: Polity. 
Finch, Janet. and Jennifer Mason. 1993: Negotiating Family Responsibilities, London: Routledge

Frericks, Patricia., Per H. Jensen., and Birgit Pfau-Effinger. 2014: "Social Rights and Employment Rights Related to Family Care: Family care regimes in Europe”, Journal of Aging Studies, 29: 66-77.

Gates, Bruce L. 1980: Social Program Administration: The Implementation of Social Policy, Prentice-Hall.

Gibbs, Graham. 2008: Analyzing Qualitative Data, 2nd edition, Sage: Thousand Oaks.

Glaser, Barney G. and Judith Holton. 2004: “Remodelling Grounded Theory”, Forum Qualitative Sozialforschung/Forum: Qualitative Social Research, 5(2).

Glaser, Barney G. and Anselm L. Strauss. 1967: The Discovery of Grounded Theory: Strategies for Qualitative Theory, New Brunswick: Aldine Transaction.

Hahm, Inhee. 2012: "Socio-Economic Crisis and 'Falling from Grace' of the Middle-Class Family in Korea”, Journal of Korean Studies, 43: 531-569. (In Korean)

Ham, Sunyu. and Bageui Hong. 2017: "The Relationship between Formal Care and Informal Care: Focusing on home care service for elderly”, Korean Journal of Social Welfare, 69(4): 203-225. (In Korean)

Han, Gyoung-Hae. and Seo-Youn Lee. 2009: "The Effect of Motivation and Social Support on Burden of Spouse Caregivers: Focused on gender differences”, Journal of the Korean Gerontological Society, 29(2): 683-699. (In Korean)

Han, Gyung-Hae. and Soon-Duck Yoon. 2000: "Failed Parental Strategy? Determinants of the living arrangements among the rural elderly in Korea”, Korean Journal of Sociology, 34, Fall: 649-669. (In Korean)

Han, Gyounghae. and Sung-Eun Yoon. 2004: "The Bilateralization of the Kinship Relation in Korean Families: Focused on the intergenerational exchange”, Korean Journal of Population Studies, 27(2): 177-202. (In Korean)

Han, Gyoung-Hae. and Jung-Yeon Son. 2009: “The Effects of Motives for Caregiving and Family Relationship Quality on Caregiving Burden/Reward of the Spouse Caregivers of Frail Elderly in Korea: A focus on gender differences.”, Journal of Korean Family Study Association, 21(2): 81-109. (In Korean)

Han, Min-A. and Gyeong-Hye Han. 2004: "Patterns of Intergenerational Support Exchange and the Psychological Well-being of Adult Children in Korea”, Journal of Family Relations, 9(1): 135-152. (In Korean)

Han, Sang-Hie. 2019: “Judiciability of the Economic, Social and Cultural Rights”, Korean Public Law, 39(1): 93-133. (In Korean)

Hobson, Barbara. 1990: "No Exit, No Voice: Women's economic dependency and the welfare state”, Acta Sociologica, 33(3): 235-250.

Hobson, Barbara. and Ruth Lister. 2002: “Citizenship”, In Babara Hobson, and Jane Lewis (eds.), Contested Concepts in Gender and Social Politics, Edward Elgar Publishing.

Hochschild, Arlie. and Anne Machung. 1990: The Second Shift, New York: Avon Books.

Hong, Kyung-Joon. and Ho-Geun Song. 2003: "Continuity and Change in Korean Welfare Regime: After 1990”, Korean Journal of Social Welfare, 55: 205-230. (In Korean) 
2006: "Continuity and Change in the Korean Welfare Regime”, Journal of Social Policy, 35(2): 247-265.

Hong, Se-young. 2011: “The Study about the Meaning of the Formal Elderly Care Labour by Employee in Long-Term Care Setting”, Journal of Welfare for the Aged, 51: 65-190. (In Korean)

Hong, Sung-Wook. 2011: "Current Issues and Future Directions in Payments for Cohabited Family Care Workers in the LTCI”, Health Insurance and Policy, 10(1): 142-152. (In Korean)

Hooyman, Nancy.R. and H. Asuman Kiyak. 2008: Social Gerontology: A Multidisciplinary Perspective (8th eds), Boston: Pearson Education, Inc.

Hosken, Fran P. 1981: “Toward a Definition of Women's Human Rights”, Human Rights Quarterly, 3(2): 1-10.

Huber, Evelyne., Johnd Stephens., David Bradley., Stephanie Moller., and Francois Nielsen. 2009: “The Politics of Women’s Economic Independence”, Social Politics: International Studies in Gender, State \& Society, 16(1): 1-39.

Huh, Ra-Keum. 2006: “Feminist Theorization for Socializing Care”, Journal of Korean Women's Studies, 22(1): 115-145. (In Korean)

Hwang, Bo-Ram. and Jeong-Hee Kang. 2015: “A Qualitative Study on Communication between Elders Living with Dementia and Their Family Caregivers and Their Quality of Life”, Korean Journal of Family Welfare, 20(1): 2749,http://dx.doi.org/10.13049/kfwa.2015.20.1.27 (In Korean)

Hwang, Ji Seon., Hey-Jung Jun., and Eun Jee Lee. 2015: “Family Caregivers’ Coping Resources and Life Satisfaction”, Family and Environment Research, 53(5): 519530, http://dx.doi.org/10.6115/fer.2015.041 (In Korean)

Hwang, Jung-Mee. 2007: “The Social Constitution of Women’s Social Rights and Multiple Position of Women as Wife, Mother and Worker”, Issues in Feminism, 7(1): 13-54. (In Korean)

Hwang, Namhee. 2016: "Policy Issues on Old-Age Income Security”, Health and Welfare Policy Forum, 239, September 2016: 21-33. (In Korean)

Hwang, Sun-Jae. and Cheong-Seok Kim. 2013: “Old-Age Inequality Decomposition”, Korean Journal of Sociology, 47(4): 201-226. (In Korean)

Im, Jinsub., Kyungrae Hyun., and Hoyong Lee. 2011: Strategies for Improving Informal Caregiving for the Development of the Long-Term Care System, Research paper, Health Insurance Policy Institute of Korea. (In Korean)

Iversen, Torben. and Anne Wren 1998: "Equality, Employment, and Budgetary Restraint: The trilemma of the service economy”, World Politics, 50(4): 507-46.

Jang, Hye-Young. and Myungsun Yi. 2017: "Hermeneutic Phenomenological Study on Caring Experience of Spouses of Elderly People with Dementia at Home”, Journal of Korean Academy Nursing, 47(3): 367-379. (In Korean)

Jegal, Hyeon-Suk. 2009: "Distorted Marketization of the Long-Term Care Service Provision”, The Radical Review, Autumn: 211-233. (In Korean) 
Jeon, Byeongjoo. and Kim Hyunsoo. 2016: "Effects of Role Stress on Service Quality of Family Care Workers and Buffering Effects of Occupational Identity”, Institute for Humanities and Social Science, 1-42. (In Korean)

Jeong, Jae-Ki. 2007: “Contact Frequency and Social Supports among Korean Kin: From the comparative perspective”, Korea Journal of Population Studies, 30(3): 157-178. (In Korean)

Jun, Hee-Kyung. 2012: Age-Gender Regime and Women's Age: Thinking The Political Implication of Ageing Experiences in Term of Narrative Time, Department of Women's Studies of the Graduate School Ewah Women's University, PhD dissertation. (In Korean)

Jun, Hey-Jung. and Kyu-Young Cho. 2011: "Transitions to Caregiving, Social Ties, and Depression among Korean Middle Aged and Elderly”, Journal of Community Welfare, 37, 2011.06: 387-411. (In Korean)

Jun, Sang-In. 2007: “Cultural Sociology of a Preference for an Apartment”, Journal of Environmental Studies, Vol. 45: 11-32. (In Korean)

Jung, Hee-Sun. 2012: “A Study on Policy and Act Making Process History of the Act on LongTerm Care Insurance for the Elderly”, Social Security Law Studies, 1: 163-187. (In Korean)

Jung, Kyunghee., Ae Cho., Younghee Oh., Youngchan Byun., Jaekwan Byun., and Hyunsang Moon. 1998: Living Profiles and Welfare Service Needs of Older Persons in Korea, Korea Institute for Health and Social Affairs. (In Korean)

Jung, Kyunghee., Younghee Oh., Jaeeun Seok., Serok Do., Chanwoo Kim., Yunkyung Lee., and Heekyung Kim. 2005: Living Profiles and Welfare Service Needs of Older Persons in Korea in 2004, Korea Institute for Health and Social Affairs. (In Korean)

2008: Living Profiles and Welfare Service Needs of Older Persons in Korea, Korea Institute for Health and Social Affairs. (In Korean)

Jung, Kyunghee., Younghee Oh., Yunkyung Lee., Changkyun Son., Bomi Park., Sooyeon Lee., Jihyun Lee., Jungdon Kwon., Soo-Bong Kim., So-Jeong Lee., Yong-Sik Lee., Yoon-Hwan Lee., Sungjae Choi., and Soyoung Kim. 2012: Living Profiles and Welfare Service Needs of Older Persons in Korea in 2012, Korea Institute for Health and Social Affairs. (In Korean)

Jung, Kyunghee., Younghee Oh., Eunna Kang., Jaeho Kim., Duk Sunwoo., Miae Oh., Yunkyung Lee., Namhee Hwang., Kyung-Rae Kim., Sinhwee Oh., Bomi Park., Hyungu Shin., and Keumryong Lee. 2014: Living Profiles and Welfare Service Needs of Older Persons in Korea, Korea Institute for Health and Social Affairs. (In Korean)

Jung, Youn. and Soonman Kwon. 2014: "Critical Appraisal from Gender Perspective on Cash Benefit Policy in the Long-Term Care”, Korean Journal of Public Health, 45(1): 15-24. (In Korean)

Kang, Hyun Sook., Keum Ja Go., Won-Ock Kim., Eun Sim Kim., Soon-Yong Khim., Hyun-Li Kim., Soon-Ok Shin., Sang-Eun Oh., Jeong-Sook Won., Chun-Yu Li., Min Jung., and Nam Hee Chef. 1999: “The Lives of Daughters-in-law Who Care for Parents with Dementia”, Journal of Korean Academy of Nursing, 29(6): 1233-1243. (In Korean)

Kang, Tae-Wha., Eun-Ja Yeun., and Mi-Soon Jeon. 2014: “The Burden Types of Primary Caregivers for a Demented Elderly Using Q Methodology”, Journal of Korean Society for the Scientific Study of Subjectivity, Vol. 28, 2014.6: 53-73. (In Korean) 
Kho, Duk-Hwan. 2009: “Assessment of the Clinical Features of Bilateral Sequential Hip Fractures in the Elderly”, Journal of the Korean Orthopaedic Association, 44(3): 369-376.

KIHASA. 2010: "Issues on the Payments for Services Offered by Family Members: A study on the compensations for service family members offer”, Presentation of the Official Meeting of KIHASA, on 6th September 2010 in Seoul. (In Korean)

Kim, Cheol-Joo. and Sung Dae Hong. 2007: “A Study on the Reforms of Long-Term Care System in OECD Countries: Focusing on the institutionalization of rewards and supports for informal care”, Journal of Welfare for the Aged Institute, 35: 231-251. (In Korean)

Kim, Cheong-Seok. and Ik-Ki Kim. 2000: "Patterns of Intergenerational Support Exchange and the Life Satisfaction of the Elderly Parents in Korea”, Journal of the Korea Gerontological Society, 20(2): 255-168. (In Korean)

Kim, Dong-Bae., Byung-Sun Yoo., and Soo-Min Shin. 2012: "The Effects of Housing Poverty on the Depression of the Elderly: The mediating effect of social service”, Journal of the Korean Gerontological Society, 32(4): 1041-1061. (In Korean)

Kim, Eun-Hee. 1995: "Family as a Cultural Construct”, Korean Cultural Anthropology, 27: 183214. (In Korean)

Kim, Hee-Kang. 2016: “Is Long-Term Care Insurance in South Korea a Socializing Care Policy”, Critical Social Policy, 36(4): 630-648. (In Korean)

Kim, Hee-Kang. and Moon-Sun Kang. 2011: "A Public Ethic of Care: Eva Kittay and the care aid program to families with disabled children in South Korea”, Korean Political Science Review, 44(4): 45-71. (In Korean)

Kim, Hye-Kyung. 2006: Formation of Modern Family and Gender in the Japanese Colonial Period, Seoul: Changbi. (In Korean)

Kim, Hye-Kyung. and Myoung-Hee Namgung. 2009: “Elderly Care by the Son’s Families”, Korean Journal of Sociology, 43(4): 180-220.

Kim, Hyunjoo. 2001: The First Son and His Wife: Thirty-three Pairs of the Oldest Son's Married Couple of Our Day Say, Seoul: Saemulgyel Press, ISBN 9788988336991.

Kim, Hye-Sook., Eun-Hee Kim., Hang-Won Kim., and Chul-In Yoo. 2005: “Meanings of CoResidence and Changing Korean Families: Case studies of urban middle class families in the Seoul area and Jeju island”, Family and Culture, 17(1): 3-34. (In Korean)

Kim, Jiyeon. and Hongsoo Kim. 2016: "Effects of Home Care Services Use by Older Adults on Family Caregiver Distress”, Journal of Korean Academy of Nursing, 46(6): 836-847. (In Korean)

Kim, Jong-Soo. 2013: "Rediscovery and Succession of Social Security Idea Go the First Constitution with 'Equal Living Rights' as the Main Theme”, Korean Social Security Law Studies, 2(2): 1-38. (In Korean)

Kim, Ju-Hyun. 2016: “Family Caregiving and Life Satisfaction of Korean Elderly Women”, Korean Journal of Population Studies, 39(2): 49-70. (In Korean)

Kim, Ju-Sung., Hae-Jung Lee., and Ki-Ryeon Kim. 2004: “Caregiving Appraisal, Burnout and Coping Strategies According to the Types of Family Relationship”, Journal of Welfare for the Aged Institute, 24(4): 39-5. (In Korean) 
Kim, Jung-Hyun. 2009: “The Effects of Cultural Values on the Physical Health of Korean American Caregivers through Caregiver Burden and Social Support, Applying to the SocioCultural Stress and Coping Model”, Journal of the Korean Gerontological Society, 29(2): 377-394. (In Korean)

Kim, Keun-Hong. 2013: “A Comparative Study on Support System for the Informal Sector on Caregiving for the Elderly”, Korean Journal of Social Welfare Education, Vol. 21: 192224. (In Korean)

Kim, Kwuy-Bun. and Kyung-Hee Lee. 1998: "Ethnography of Caring Experience for the Senile Dementia”, Journal of Korean Academy of Nursing, 28(4): 1047-1059. (In Korean)

Kim, Kyung-Hee. 2009: “A Study on the Reproducing Process of Gendered and Low-waged Care Work: Focused on care work in the informal sector", The Journal of Asian Women, 48(2):147-184. (In Korean)

Kim, Kyounghee. and Eun-ae Kang. 2010: "The Effects of Family Care Responsibility on Wage of Men and Women”, The Journal of Asian Women, 49(2): 121-155. (In Korean)

Kim, Mi-Kyung. 2000: "Sociological Approach of Family for the Study of the Elderly's Welfare: Women's role for caring the elderly”, Korean Journal of Sociology, 34(SPR), Mar. 2000: 65-84. (In Korean)

Kim, Sang-Wook. 2000: "Eldercare Attitude and Behavior (III): Development and estimation of a path model”, Korean Journal of Sociology, Vol. 34: 1037-1067. (In Korean)

Kim, Soon-Yang. 2007: "Improving the Delivery System Linkage of the National Long-Term Care Insurance for the Elderly”, Korean Social Policy Review, 14(1): 82-122. (In Korean)

Kim, Song-Yi. 2012: “A Study on the Labor Experiences of Care Service Workers: Focused on emotional labor and relation-based labor”, The Women's Studies, 81(1): 103-36. (In Korean)

Kim, Tae-Hong., Bok-Tae Kim., Ho-Joong Bae., Heung-Sung Nho., and Sang-Jun Lee. 2011: Economic Growth Strategy and Job Creation for Women in Service Sector, Seoul: Korean Women's Development Institute. (In Korean)

Kim, Tae-Hyun. and Ji-Hyeon Park. 2014: “A Study on Long-Term Care Insurance Service of Elderly Women Spouse Caregiver and Implication of Policy Direction: Focused on psychological well-being and marital quality of elderly women”, Journal of Family Relations, 9(2): 169-185. (In Korean)

Kim, Tae-Hyun. and Gil-Yang Jun. 1995: “A Study on the Family Caregiving Experiences of the Demented Elderly Persons”, Journal of the Korea Gerontological Society, 1: 15-27. (In Korean)

Kim, Yeong-Soon. 2010: "The Social Rights of Female Non-regular Workers and the Gender Regimes in Korea”, Korean Social Security Studies, 26(1): 267-287. (In Korean)

Kim, Yong-Hee. and Jeong-Shin An. 2012: "Stress, Life Satisfaction and Service Satisfaction of Elderly and Elderly Family Using Long-Term Care Service”, Journal of Welfare for the Aged, Vol. 57: 161-190. (In Korean)

Kim, Young-Ock. and Young-suk Kim. 2011: "Measuring Family Caregiving Output for the Elderly”, The Women's Studies, 80(1): 181-215. (In Korean) 
Kim, Young-Ock., Hyung-Joo Min., and B. Kim. 2006: The Polarization of the Korean Women's Labor Market, Seoul: Korean Women’s Development Institute. (In Korean)

Kim, Young-Ock., Sun-Haeng Lee., and Min-Soo Kim. 2012: The Trends of Female Labor Market after 2000: Focused on the Reduction of the Female Self-Employed Workers, Seoul: Korean Women's Development Institute. (In Korean)

KLI. 2010: Improving Job Creation Capability of the Services Industry by Upgrading the Employment Structure (II): with Focus on Culture and Tourism Sectors, Seoul: Korea Labor Institute. (In Korean)

Knijn, Trudie. and Monique Kremer. 1997: "Gender and the Caring Dimension of Welfare States: Toward inclusive citizenship”, Social Politics: International Studies in Gender, State \& Society, 4(3): 328-361.

Kodate, Naonori. and Virpi Timonen. 2017: "Bringing the Family in through the Back Door: The stealthy expansion of family care in Asian and European long-term care policy”, Journal of Cross-Cultural Gerontology, 32: 291-301. DOI 10.1007/s10823-017-9325-5

Kohli, Martin. 2007: “The Institutionalization of the Life Course: Looking back to look ahead”, Research in Human Development, 4(3-4): 253-271.

Komter, Aafke. 2004: “Some Darker Sides of Family Solidarity”. In Knijn, Trudie. and Komter, Aafke. (eds), Solidarity Between the Sexes and the Generations: Transformations in Europe, Cheltenham: Edward Elgar, 133-49.

Kong, Byung-Hye. 2002: “A Philosophical Inquiry on the Moral Horizon of Care in Nursing”, Korean Feminist Philosophy, 2(6): 43-69. (In Korean)

2010: “Elderly Care in Korean Society”, Korean Feminist Philosophy, 13: 1-22. (In Korean)

Kong, Seonhee. 2013: “The Elderly's Changing Expectations of Family Care and Their Desire for the Care Policy”, Korean Journal of Sociology, 47(1): 277-312. (In Korean)

KOSIS. 2017: Apartment, Statistical Terminology in Korean Statistical Information Service, https://meta.narastat.kr/metasvc/index.do?iemInputNo=0000139689127 (Accessed on March 27. 2018)

KOSTAT. 2013: Household Income and Expenditure Survey, Statistics Korea, http://kostat.go.kr/portal/korea/kor_nw/2/4/3/index.board (Accessed on June 27. 2018)

2017a: Population and Housing Census, Statistics Korea, http://kostat.go.kr/policy/quality/qt_dl/6/index.board?bmode=read\&aSeq=365743(Accesse d on March 27. 2018)

2017b: Senior Citizen Statistics, Statistics Korea

http://kostat.go.kr/portal/korea/kor_nw/2/1/index.board?bmode=read\&bSeq=\&aSeq=36336 2\&pageNo=1\&rowNum $=10 \&$ navCount $=10 \&$ currPg $=\&$ sTarget $=$ title\&sTxt $=($ Accessed on June 26. 2018)

2018: Households' Finances and Welfare Statistics, Statistics Korea. http://kostat.go.kr/portal/korea/kor_nw/2/4/4/index.board (Accessed on June 26. 2018).

Kremer, Monique. 2007: How Welfare States Care: Culture, Gender, and Parenting in Europe, Amsterdam University Press. 
Kröger, Teppo. 2001: “Comparative Research on Social Care: The state of the art”, SOCCARE Project Report 1, Bruxelles: European Commission.

Kwon, Soo-Hyun. 2013: Social Specificities of Care Labor in South Korea: Focusing on Gendered Relational Labor, Yonsei University, Doctoral Thesis. (In Korean)

Kymlicka, Will. and Wayne Norman. 1994: "Return of the Citizen: A survey of recent work on citizenship theory”, Ethics, 104(2): 352-381.

Lee, Dong-Yeon. 2010: “Korean Daily Life and the Cultural Habitus”, Culture and Science, Vol. 61: 169-195. (In Korean)

Lee, Dongok. 2011: “A Research on Women’s Experience about the Care for Elderly Parents”, PNU Journal of Women's Studies, 21(1): 83-122. (In Korean)

Lee, Duksik. 2015: “A Study Hyo and Care in Familialism”, Journal of Social Science, 32(2): 173-209, Chungbuk National University Social Science Institute. (In Korean)

Lee, Eun-Hwa. 2008: A Study on the Policy-Making Process of Long-Term Care Insurance in Korea: Focused on the Participants' Role, Social Welfare department of the Graduate School of Pyoengtaek University, PhD dissertation. (In Korean)

Lee, Hong-Sa. 2012: "Caregiver Burden in Caring for Elders before and after Long-Term Care Service in Korea”, Journal of Korean Academy of Nursing, 42(2): 236-247. (In Korean)

Lee, Hye-Kyung. 2004: "Welfare Reforms in Post Crisis Korea: Dilemmas and choices”, Social Policy and Society, 3(3): 291-99. (In Korean)

Lee, Hyun-Joo. 2006: "Caregiving Experience and Adaptation Process of the Husbands Who Are Caring for Wives with Alzheimer: Focusing on elderly couple household”, Journal of the Korean Gerontological Society, 26(1): 45-62. (In Korean)

Lee, Hyunju. 2015: “A Study on the Gender Inequality of Elderly Women’s Household and Caregiving Labor”, PNU Journal of Women's Studies, 25(3): 141-177. (In Korean)

Lee, Hyunsook. 2011: “A Comparative Study on Family Care in Korea and Scotland”, Health and Social Welfare Review, 31(4): 345-381. (In Korean)

Lee, Hyun-Joo., Soung-Yee Kim., and Hyun-Za Ku. 2007: “A Phenomenological Study on the Caregiving Experience of the Husbands Who Are Caring for Wives with Presenile Dementia”, Korean Journal of Family Social Work, 21(0): 1-39. (In Korean)

Lee, In-Jeong. 2006: "Comparison of the Determinants of Caregiving Burden between Wives and Daughters-in-law of the Impaired Older Persons”, Korean Journal of Social Welfare Studies, 31: 161-193. (In Korean)

Lee, Jae-Kyung. 1999: “Modern Transformation of Korean Family: Feminist analysis”, Journal of Korean Women's Studies, 15(2): 55-86. (In Korean)

Lee, Jaerim. 2015: “Institutional Familialism in Child and Elderly Care Policies”, The Korean Home Management Association, Conference paper 5: 78-89. (In Korean)

Lee, Jae Kyung. and Hye-Gyung Park. 2011: “Measure of Women’s Status and Gender Inequality in Asia: Issues and challenges”, Asian Journal of Women's Studies, 17(2):48-72. (In Korean)

Lee, Jinsook. 2014: "Does the Long-Term Care Insurance for the Elderly Ease Family Care Burden?”, Family and Culture, 26(2): 126-150. (In Korean) 
Lee, Jin-suk. and Jinhwa Park. 2011: "Effectiveness Evaluation of Long-Term Care Service for the Elderly through the Diffusion of Market Principle”, Health and Social Welfare Review, 31, Vol. 4: 5-33. (In Korean)

Lee, Ju-Eun. and Myung-Suk Park. 2012: “A Study on an Improvement Plan for Elderly Welfare System in Korea: Focusing on elderly welfare system in germany”, The Journal of Eurasian Studies, 9(4), December 2012: 379-412. (In Korean)

Lee, Kang-Oh. 1999: “A Study on Stress and Health of Family Caregivers Caring for Demented Elderly”, Journal of Korean Academy of Psychiatric and Mental Health Nursing, 8(2): 384-398. (In Korean)

Lee, Kyu-Sik. 2006: "Problem with and Policy Agenda for Long-Term Care”, Health Policy Forum, 4(1): 120-127. (In Korean)

Lee, Mee-Ae. 2009: “Rural-urban Differences in Factors Affecting Caregivers’ Burden of Impaired Elders in Korea”, Journal of Welfare for the Aged, 44: 71-88. (In Korean)

Lee, Min-Sook. 2012: The Caregiving Experience of Family Caregiver Who Supports a Family Member Recognized as the Beneficiary of the Long-Term Care Insurance for the Aged, Department of Social Welfare Graduate School, Kyunggi University. (In Korean)

Lee, Seong-Lim. 2015: "Income Composition and Expenditure Inequality of the Elderly Household by Income Classes”, Korea Journal of Population Studies, 38(2): 85-111. (In Korean)

Lee, Suk-Jin. 2011: “Institutionalization of Care Labor and Differences among Women”, Feminism Study, 11(2): 49-83. (In Korean)

Lee, Sung-Hoon. 2010: “Who and How Social Rights?”, Jeju Human Rights Conference, Jeju. (In Korean)

Lee, Yun-Kyung. 2009: "Factors of Long-Term Care Service Use by the Elderly”, Health and Social Welfare Review, 29(1): 213-35. (In Korean)

2010: "Development of Reward System for Family Carer in Elderly Long-Term Care Insurance”, Health and Welfare Forum, (Monthly) 2010. July: 96-104. (In Korean)

Lewis, Jane. 1992: “Gender and the Development of Welfare Regime”, Journal of European Social Policy, 2(3): 159-73.

2003: “2. The Application of Qualitative Methods.” In Ritchie, Jane and Jane Lewis (eds,), Qualitative Research Practice: A Guide for Social Science Students and Researchers, , 47-76. Thousand Oaks, Calif.; London: Sage.

Lewis, Jane. and Barbara Meredith. 1988: “Daughters Who Care”, Daughters Caring for Mothers at Home, London: Routledge.

Li, Mei., Yang Zhang., Zhenyu Zhang., Ying Zhang., Litao Zhou L., and Kun Chen. 2013: "Rural-urban Differences in the Long-Term Care of the Disabled Elderly in China", PLoS ONE, 8(11): e79955. doi:10.1371/journal.pone.0079955

Lim, Byungwoo. and Sungyul Park. 1999: "The Sociological Context of Community Care for Elderly People”, Journal of Agricaultural Education and Human Resource Development, 13(1): 87-96. (In Korean)

Linton, Ralph. 1936: The Study of Man, 113-131, New York: Appleton-Century. 
Lister, Ruth. 1997: Citizenship: Feminist Perspectives, Basingstoke: Palgrave Macmillan.

Lutz, Helma. and Ewa Palenga-Möllenbeck. 2007: “Care Workers, Care Drain, and Care Chains: Reflections on care, migration, and citizenship”, Social Politics: International Studies in Gender, State \& Society, 19(1): 15-37.

Min, Kyung-Hee. 2002: "Workforce”, in Duseop Kim, Sangtae Kim, and Kisoo Eun (eds.), Population in Korea, : 393-428. Seoul: Kostat. (In Korean)

Ma, Kyung-Hee. 2011: “Universal Welfare State and Caring”, Issues in Feminism, 11(2): 85-116. (In Korean)

Malhotra, Anju. and Sidney Ruth Schuler. 2005: “Women’s Empowerment as a Variable in International Development”, in Deepa Narayan (ed.), Measuring Empowerment: CrossDisciplinary Perspectives, 71-88. Washington DC: The World Bank.

Marrs, Lisa. 2011: The Sandwich Generation: The Challenges of Caring for an Elderly Parent and Raising Children, Diss. California State University, Long Beach.

Marshall, Thomas Humpry. 1950: Citizenship and Social Class: And Other Essays, Cambridge: University Press.

1954: “Note on Status”, in Class, Citizenship and Social Development: Essays by T.H Marshall, (1964): 200-208. New York: Doubleday \& Company, Inc.

Matthews, J. Scott. and Lynda Erickson. 2005: "Public Opinion and Social Citizenship in Canada”, Canadian Review of Sociology/Revue Canadienne de Sociologie, 42(4), 373-401.

Mey, Günter. 2000: “Erzählungen in Qualitativen Interviews: Konzepte, probleme, soziale Konstruktionen”, In Sozialer Sinn, 1(1): 135-51. URL: http://nbnresolving.de/urn:nbn:de:0168-ssoar-4471

Milligan, Christine. and Janine Wiles. 2010: “Landscape of Care”, Progress in Human Geography, 34(6): 736-54.

Min, Kyung-Hee. 2002: “Workforce”, in Duseop Kim., Sangtae Kim., and Kisoo Eun (eds.). Population in Korea: 393-428. Seoul: Kostat. (In Korean)

Ministry of Education. 1975: Statistical Yearbook of Education, Seoul: Korean Educational Development Institute. (Korea)

Moen, Phyllis. and Nicole DePasquale. 2017: "Family Care Work: A policy-relevant research agenda”, International Journal of Care and Caring, 1(1): 45-62.

Morgan, David.H.J. 1996: Family Connections, Cambridge: Polity Press.

1999: "Risk and Family Practices: Accounting for change and fluidity in family life", in Elizabeth B. Silvia and Carol Smart (eds.), The new family? London: Sage.

Na, Hang-Jin. 2003: “A Study on the Role of Leisure of the Elderly for Upgrading of the Quality of Life”, Journal of the Korea Gerontological Society, 23(4): 53-70. (In Korean)

Namgung, Myoung-Hee. 2009: “Analytic Review on the Family Care Relations Research \& Propositions: Focused on the relationship between the aged parents and their adult married children in Korea”, Journal of Social Science, 20(3): 49-79. (In Korean) 
2010: The Practice and Negotiation of the Elderly Parents Caring: A Qualitative Study from the 'Relational Autonomy' Perspective, Department of Sociology, the Graduate School of Chonbuk National University, PhD dissertation. (In Korean)

National Institute of the Korean Language. http://www.korean.go.kr (Access on October 1st. 2015)

National Health Insurance Corporation (NHIC). 2009: Long-Term Care Statistics Annual Report, National Health Insurance Service. (In Korea)

2011: Heath Insurance \& Policy, 10(1), Seoul: NHIC.

2012: Long-Term Care Insurance Statistical Yearbook 2011. Seoul: NHIC.

National Health Insurance Service (NHIS). 2012: Long-Term Care Statistics Annual Report, National Health Insurance Service. (In Korea)

2018: Long-Term Care Statistics Annual Report, National Health Insurance Service. (In Korea)

2018: Long-Term Care Statistics Annual Report, National Health Insurance Service. (In Korea)

Nelson, Debra L. and Susan Michie. 2004: "Women in Management in the USA”, in Davidson, Marilyn J. and Ronald J. Burke (eds.), Women in Management Worldwide: Facts, Figures and Analysis.,. :195-208. Burlington: Ashgate.

OECD. 2013: OECD Social Expenditure Database, http://stats.oecd.org/index.aspx (accessed 30 March 2013)

2015a: Social Expenditure Database, http://www.oecd.org/social/expenditure.htm

(Accessed on June. 08. 2016.)

2015b: Pension at a Glance 2015, OECD.

2017: Pension at a Glance 2017, OECD.

2018: OECD Labour Force Statistics 2017, OECD

Publishing. DOI:http://dx.doi.org/10.1787/oecd_lfs-2017-en

Offe, Claus. 1992: “A Non-Productivist Design for Social Policies”, Arguing for Basic Income, VERSO, London: 61-78.

Oh, Eunjin. and Eunyoung Roh. 2010: "Impact of Institutionalization of Care Services on the Woman's Workforce: Focused on the comparison between care helpers for the elderly and caregivers for the sick”, The Journal of Asian Women, 49(2): 185-216. (In Korean)

Oh, Se-Geun. 2010: “A Study on the Current State of Long-Term Caregivers' Caring Activities and Policy Recommendations for the Improvement of Long-Term Caregivers' Working Condition: A focus on the discourse of 'decent work'”, Korean Journal of Social Research, 2: 101-136. (In Korean)

Paik, Jina. 2015: “Experiences of Elderly Female Caregivers of Spouses”, The Korean Journal of Humanities and the Social Sciences, 29(4): 93-121. (In Korean)

Park, Ha-Jung. 2008: Study on the Policy Network of Social Welfare Policy Making: Focused on the Long-Term Care Insurance for the Elderly and Law-Making on Welfare for the Elderly, $\mathrm{PhD}$ thesis in the faculty of administrative science in Kyunghee University. (In Korean) 
Park, Keong Suk. and Mi Seon Kim. 2016: "The Impact of Changes in Household Living Arrangements on the Changes in Poverty Rate among Elderly People in South Korea”, Korean Journal of Sociology, 50(1): 221-253. (In Korean)

Park, Ki-Nam. 2013: “Women’s Care Role and Individualization from the Perspective of Life Course: Focusing on relation of the national public pension”, Issues in Feminism, 13(1): 47-91. (In Korean)

Park, Kyung-Suk. and Young-Hye Kim. 2005: "Meanings of Family and Work Constructed from the Narratives of Women's Life”, Economy and Society, Vol. 68: 133-160. (In Korean)

Park, Mee-Hae. 1991: "Patterns and Trends of Educational Mating in Korea”, Korean Journal of Population and Development, 20: 1-15. (In Korean)

Pateman, Carole. 1992: "Equality, Difference, Subordination: The politics of motherhood and women's citizenship”, Beyond Equality and Difference: Citizenship, Feminist Politics and Female Subjectivity, 17-31.

Peach, Lucinda Joy. 2011: “Are Women Human? The promise and perils of women's rights as human rights.” In Lynda S. Bell, Andrew Pearson J. Nathan and Ilan Peleg (eds.), Negotiating Culture and Human Rights: 153-196. New York: Columbia University Press.

Peng, Ito. 2002: "Social Care in Crisis: Gender, demography, and welfare state restructuring in Japan”, Social Politics: International Studies in Gender, State \& Society, 9(3): 411-443. 2010: "The Expansion of Social Care and Reform: Implications for care workers in the Republic of Korea”, International Labour Review, 149(4): 461-476.

2011: "The Good, the Bad and the Confusing: The political economy of social care expansion in South Korea”, Development and Change, 42(4): 905-23.

2014: "The Social Protection Floor and the 'New' Social Investment Policies in Japan and South Korea”, Global Social Policy, 14(3): 389-405. DOI:

$10.1177 / 1468018114533711$

Peng, Ito. and Sue Yeandle. 2017: Eldercare Policies in East Asia and Europe: Mapping Policy Changes and Variations and Their Implications, Discussion Paper at Progress of the World's Women 2018, UN Women.

Pfau-Effinger, Birgit. 2004: Development of Culture, Welfare States and Women's Employment in Europe, Aldershot: Ashgate. 2005a: "Culture and Welfare State Policies: Reflects on complex interrelation”, Journal of Social Policy, 34(1): 1-20.

2005b: "Welfare State Policies and the Development of Care Arrangements”, European Societies, 7(2): 321-347.

Pfau-Effinger, Birgit., Ralf Och., and Per H. Jensen. 2011: “Tensions between 'Consumerism’ in Elderly Care and the Social Rights of Family Carers: A German-Danish comparison”, Nordic Journal of Social Research 2.

Pijl, Marja. and Clarie Ramakers. 2007: “Contracting One’s Family Members: The dutch care allowance”, In Ungerson, Clare. and Susan Yeandle (eds), Cash for Care Systems in Developed Welfare States, London: Palgrave, 81-103. 
Pinquart, Martin. and Silvia Sörensen. 2011: "Spouses, Adult Children, and Children-in-law as Caregivers of Older Adults: A meta-analytic comparison”, Psychology and Aging, 26(1): $1-14$.

Razavi, Shahra. 2007: "The Political and Social Economy of Care in a Development Context”, Gender and Development Programme Paper 3.

Reid, Catherine, E., Simon Moss., and Graeme Hyman. 2005: "Caregiver Reciprocity: The effect of reciprocity, carer self-esteem and motivation on the experience of caregiver burden", Australian Journal of Psychology, 57(3): 186-196.

Reinharz, Sulamit. and Lynn Davidman. 1992: Feminist Methods in Social Research, New York: Oxford University Press.

Rosenthal, Gabriele. 1995: Erlebte und Erzählte Lebensgeschickte: Gestalt und Struktur Biographischer Selbstbeschreibungen, Frankfurt am Main; New York: Campus Verlag. 2011: Interpretative Sozialforschung: eine Einführung, LUGAR: Beltz Juventa. 2018: Interpretive Social Research: An introduction, Göttingen: Universitätverlag Göttingen.

Rubin, Herbert J. and Irene S. Rubin. 1995: Qualitative Interviewing: The art of hearing data, Thousand Oaks, London, New Delhi, Sage.

Ryu, Im-lyang. 2013: "The Role and Occupational Status of Institutionalized Care Workers: Focused on in-home long-term care workers”, Issue in Feminism, 17(2): 189-231. (In Korean)

Sainsbury, Diane. 1996: Gender, Equality and Welfare States, Cambridge Univ. Press.

Saraceno, Chiara. 2016: "Varieties of Familialism: Comparing four southern European and East Asian welfare regimes”, Journal of European Social Policy, 26(4): 314-326.

Schütze, Fritz. 1983: “Biographieforschung und Narratives Interview”, Neue Praxis, 13(3): 283293.

Seo, In-Soon. 2003: "The Experiences of Daughters-in-law Taking Care of Their Demented Mothers-in-law: Narrative inquiry”, Journal of Qualitative Research, 4(2): 74-86 (In Korean)

Seok, Jae-Eun. 2006: "Patterns of Cash Payments for Care: Cross-national comparative study", Korean Journal of Social Welfare, 58(2): 273-302. (In Korean)

2010: "The Improvement of Long-Term Care Insurance from the Persepective of Service Provider”, Health and Welfare Forum, 2010 Oct.: 34-44. (In Korean)

Seomun, Junhee. and Keunhong Kim. 2013: “A Study on the Caring Behaviors and Physical ·Psychological Social Characteristics of Elderly Caregivers”, Journal of Community Welfare, Vol. 47: 283-306. (In Korean)

Seomun, Jinhee. and Yeo-Joo Jung. 2011: “A Qualitative Study on Caregivers' Burden Experiences for the Long-Term Care Qualified Elderly”, Journal of Institute for Social Sciences Chungnam National University, 22(4): 3-30. (In Korean)

Shin, Jang-Sup. and Ha-Joon Chang. 2003: Restructuring 'Korea Inc.': Financial Crisis, Corporate Reform, and Institutional Transition, Routledge Curzon. 
Shin, Kyung-Ah. 2011: "Defamilization of Elderly Care and the Experiences of the Aged", Korean journal of Sociology, 45(4): 64-96. (In Korean)

2013: “Marketized Individualization and Welfare Needs”, Economy and Society, 2013.6, 266-303. (In Korean)

Silverman, David. 2005: Doing Qualitative Research: A Practical Handbook, London: Sage.

Sohn, Yong-Jin. 2012: “The Effect of the Baby-Boomer Generation's Intimacy Factors with Aged Parents on Filial Responsibility after the Introduction of the Long-Term Care Insurance”, GRI REVIEW 14(2), Gyeonggi Research Institute, 2012.8: 157-186. (In Korean)

Song, Da-Young 2003: “Factors Affecting Family Caregivers' Preference for Utilazation of Community Eldercare Services”, Korean Journal of Social Welfare, Vol. 53: 105-128. (In Korean).

Song, Young-Huem. 2012: "Research about the Elderly's Job and a Relationship with the Caring Welfare in the Elderly Society”, Korean Academy of Social Welfare Support, 7(1), March: 173-191. (In Korean)

Sudarkasa, Niara. 1986: “The 'Status of Women’ in Indigenous African Societies”, Feminist Studies, 91-103.

Stacey, Clare. L. 2011: The Caring Self: The Work Experiences of Home Care Aides, New York: Cornell University Press.

Strauss, Anselm. and Juliet M. Corbin. 1998: Basics of Qualitative Research: Techniques and Procedures for Developing Grounded Theory, 2nd. ed. Sage Publications, Thousand Oaks, CA, USA.

Taylor-Gooby. P. 2004: "New Risks and Social Change”. Taylor-Gooby, Peter. (eds.). New Risk, New Welfare: The Transformation of the European Welfare State, Oxford: Oxford University Press.

Tennstedt, Sharon L., Sybil Crawford., and John B. McKinlay. 1993: "Determining the Pattern of Community Care: Is co-residence more important than caregiver relationship?”, Journal of Gerontology, 48: 74-83.

The Ministry of Health and Welfare (Korea). 2005: Introduction of the Long-Term Care Insurance in Korea, Press release in Mar.23.2005. (In Korean)

2007: Improvement of the National Pension Fund Management and Supervision System, September 13th, 2007. http://www.mohw.go.kr/react/policy/policy_bd_vw.jsp (Accessed June 29th 2018)

2008: Announcement on Long-Term Care Pay Expenses etc., the announcement of the Ministry of Health and Welfare No. 2008-15, on February 2nd. 2008. (In Korean)

Theobald, Hildegard. 2012: "Home-based Care Provision within the German Welfare Mix”, Health and Social Care in the Community, 20(3): 274-282.

The Oxford English Dictionary. 2018: https://en.oxforddictionaries.com/definition/old_age (Accessed on May. 17th, 2018)

The National Health Insurance System. 2009: Statistics on National Health Insurance. (In Korean) 
The United Nations. 2015: World Population Prospects.

https://esa.un.org/unpd/wpp/publications/files/key_findings_wpp_2015.pdf (Accessed on Juli 03. 2016)

2017: World Population Prospects, the 2017 Revision, 2017. 7, http://esa.un.org/unpd/wpp (Accessed on June, 29th 2018)

The UNDP. 2014: Human Development Report Technical notes in Human Development Report 2014 http://hdr.undp.org/en/2014-report/download(Updated in 20.04. 2015.)

Tronto, Joan C. 1993: Moral Boundaries: A Political Argument for an Ethic of Care, New York: Routledge.

2001: “An Ethic of Care” in Holstein, Martha (ed.), Ethics in Community-Based Elder Care: 60-68, New York: Springer Publishing.

Tronto, Joan C. and Berenice Fisher. 1990: “Toward a Feminist Theory of Caring”. In E. Abel, \& M. Nelson (eds.), Circles of Care (pp. 36-54). Albany, NY: SUNY Press.

Ungerson, Clare. 2000: "The Commodification of Care: Current policies and future politics” in Hobson, Barbara (ed.), Gender and Citizenship in Transition, New York: Routledge.

2004: "Whose Empowerment and Independence? A cross-national perspective on 'cash for care’ schemes”, Ageing \& Society, 24: 189-212.

2005: Morals and Politics in "Payments for care: An Introductory Note", in Evers, Adalbert, Marja Pijl, and Claire Ungerson (eds.), Payments for care. Vienna: Avebury.

Walby, Sylvia. 1994: “Is Citizenship Gendered?”, Sociology, 28(2): 379-395.

Wang, Hye-Suk. 2013a: Familial Foundations of the State Welfare: A Comparative Approach to the National Health Insurance Program in South Korea and Taiwan, Doctoral Diss, Yonsei University. (In Korean)

Wang, Hye-Suk. 2013b: "Recognition Struggles in Welfare Politics: Focused on the dependent system of the NHI in S. Korea”, Korean Journal of Sociology, 47(4): 67-106. (In Korean)

Witzel, Andreas. 1985: “Das problemzentrierte Interview“, In Jüttemann, Gerd (ed.): Qualitative Forschung in der Psychologie. Grundfragen, Verfahrensweisen, Anwendungsfelder. Weinheim:Beltz. 227-255

2000: “The Problem-Centred Interview [26 paragraphs]”, Forum Qualitative Sozialforschung/Forum: Qualitative Social Research, 1, 1, Art. 22, available at: http:// nbnresolving.de/urn:nbn:de:0114-fqs0001228, Access 07 December 2010.

Witzel, Andreas. and Herwig Reiter. 2012: The Problem-Centred Interview: Principles and Practice, Los Angeles: Sage.

Woo, Myoung-Suk. 2005: "Reinterpreting Early Welfare Policies in Korea: An analytical value of institutional perspectives”, Economy \& Society, 67: 226-250. (In Korean)

2006: "Women's Economic Status and the Role of the State in Korea: Focusing on the discussion of the state autonomy”, Journal of Korean Society, 40(3): 62-90. (In Korean)

Wood, Geof. and Ian Gough. 2006: “A Comparative Welfare Regime Approach to Global Social Policy”, World Development, 34(10): 1696-1712. 
Won, Seok-Jo. 2006: Social Welfare Policy, 7ed. Seoul: Knowledge Community. ISBN 979-116105-000-3. (In Korean)

World Economic Forum. 2014: Global Gender Gap Report 2014, World Economic Forum. http://www.weforum.org/.

Yang, Jae-Jin. 2003: "Labor Market Flexibility and the Korean Welfare State: Coping with the mismatch between the labor market and the social welfare system”, Korean Political Science Review, 37(4): 403-428. (In Korean)

Yang, Nan-Joo. 2013: “A Study on the Emergence of Family-Care worker: Why families choose to be care worker in Korea”, Korean Social Policy Review, 20(2): 97-129. (In Korean)

Yang, Nan-Joo. and In-Hee Choi. 2013: “The Impact of the Korean Long-Term Care Insurance System on its Family Caregivers: Focusing of family caregiving arrangement”, Korean Journal of Social Welfare Studies, 44(3): 31-56. (In Korean)

Yang, Seungeun. 2012: A Comparative Cohort Study on the Experiences of Job Loss and Job Seeking in Korea, Korean Journal of Human Ecology, 12(1): 41-58. (In Korean)

Yang, Yeung-Ja. 2018: “An Autoethnography on Experiences of Caring for Elderly Parents”, Korean Journal of Qualitative Research in Social Welfare, 12(2): 5-37. (In Korean)

Yi, Hee-Young. 2005: "Reconstruction of Biography as a Sociological Method: The theoretical meaning and the methodological principles regarding the theory of action”, Korean Journal of Sociology, 39(3): 120-148. (In Korean)

Yih, Bong-Sook., Chun-Mi Kim., and Myungsun Yi. 2004: "Women Caregivers' Experiences in Caring at Home for a Family Member with Dementia: A feminist approach”, Journal of Korean Academy of Nursing, 34(5): 881-890. (In Korean)

Yim, Euneui. and Eunjoo Park. 2011: The Experience of Employment Courses in the Social Works for Women in Korea, Labor Studies, 21: 2-39. (In Korean)

Yeo, Eu-Gene. 2013: “Old-age Poverty by Household Type and Policy Efforts to Reduce it” Journal of Critical Social Welfare, No. 40, August 2013: 185-219. (In Korean)

Yoon, Hyun-Sook. and Sam-Hee Ryu. 2007: "Factors Associated with Family Caregivers' Burden of Frail Elders: Comparing spouse with adult children”, Journal of the Korean Gerontological Society, 27(1): 195-211. (In Korean)

Yoon, Minseok. 2016: The Characteristics of Working Elderly in Seoul and Policy Directions, The Seoul Institute, Policy Report, Vol. 209, 2006.3: 1-22. (In Korean)

Yun, Seung-Hee. 2012: “Comparing Child Care Regimes in Welfare States: Care culturalinstitutional approach”, Korean Journal of Family Social Work, 36(6): 31-59. (In Korean)

Zelditch, Morris. Jr.1968: “Social Status”, In Sills, David L. (ed.), International Encyclopaedia of the Social Sciences, New York: Macmillan. 


\section{Appendix 1. Contact Letter (in Korean) \\ 연구참여자 모집 안내문}

안녕하세요,

저는 독일 괴팅엔 대학교에서 사회정책을 전공하고 있는 박사과정 학생입니다. 학위논문으로 "가족요 양보호사의 경험'에 대해 준비하고 있습니다. 고령사회에서 증요성이 높아진 노인장기요양보험 시스 뎀을 가족수발자의 경혐을 동해 살펴보고 이들을 지원할 수 있는 사회정책적 방안을 찾고자 하는 목적 을 갖고 있습니다. 가족을 돌보는 요양보호사로서 자신의 경험을 말씀해 주실 인터뷰 대상자를 찾고 있습니다. 인터뷰 대상자의 조건은 다음과 같습니다.

노인장기요양보혐의 요양보호사 자격을 취득하여

노인장기요양보혐 등급을 받은 가족을 돌보고 있는 분,

모든 성별, 모든 연령이 가능합니다.

해당 경험이 있으신 분들은 제가 드리는 질문에 대해 편안하게 답하시면 되고, 별다른 준비는 필요없 습니다. 약 1 시간에서 1 시간반 정도의 시간이 소요되며, 원하시는 장소와 시간에 맞추어 제가 찾아빕 젰습니다.

본인이 대상자의 조건이 되거나, 주위에 아는 분이 있으시면 연락주시기 바랍니다.

면담의 내용은 녹취기록되며 연구자가 정리한 면담 내용은 서면을 통해 확인할 수 있습니다. 연구참여 자의 이름과 신변정보는 가명으로 인용되며, 심층면접 인터뷰는 학술 목적으로만 사용됩니다. 또한 모든 자료는 익명으로 처리되며, 인터뷰 대상자의 프라이버시와 대화 내용은 괴딩엔대학교의 연구윤 리규정에 준하여 철저하게 비밀이 보장됩니다.

본 연구에 참여하신다면 자신의 경험에 대한 보다 진전된 이해와 더불어 학술 연구 진홍에 기여할것이 라 확신합니다. 참여하시는 분들께는 약소하지만 소정의 사례(2만원)도 지급됩니다.

지도교수 : Ilona Ostner Professor of Georg-August University in Gottingen iostner@uni-goettingen.de 연락처 : 010-2038-3724, taeyyun@gmail.com 


\section{Contact Letter (translated into English)}

Hello,

I am a PhD student and study social policy at the Georg-August University in Göttingen in Germany. I do a research project for my PhD dissertation on 'the experiences of the gajokyoyangbohosa (family care helper, $\mathrm{FCH}$ )'. In an ageing society, where the long-term care insurance system becomes more important, my research project aims to investigate on the experiences of family caregivers and.to find implications for social policy which supports the family caregivers.

- The requirements of the interviewee are as follows:

- Obtained eligibility for the long-term care insurance for the elderly,

- A person who cares for a family who has a long-term care insurance for the elderly.

- All genders, all ages are available.

If you have any experience, please feel free to answer my questions and do not need any preparation. The interview takes about 1 hour to 1 hour and a half, and I will meet you at the time and place you want.

If you are those who, or if you know someone around you, please contact us.

The contents of the interview are recorded, and the contents of the interviews are arranged by the researcher can be confirmed in writing. The names and personal information of research participants are cited as aliases, and in-depth interview interviews are used for academic purposes only. All materials are anonymous, and the privacy and conversation of research participants are strictly confidential in accordance with Göttingen University's Code of Ethics. 


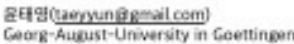

\section{연구참여 동의서(참여자 제공/보관용)}

본인은 이 연구에 자발적으로 참여하였습니다. 연구의 목적과 내용에 대한 설명을 들 어 알고 있으며, 면접에 응할 것을 허락합너다.

본인은 연구자의 대화 내용은 녹취를 동해 연구자료로 사용될 것을 알고 동의하였습니 다. 면담 동안 말한 내용을 연구자의 녹취 기록물을 보고 확인할 수 있으며 연구가 끝나면 녹 음된 내용이 지워진다는 것을 듣고 동의합니다. 연구과정 중 드러난 신변 정보는 연구 걸과물 에 직접적으로 인용되지 않으며 연구 결과물이 발표되기 전에 나에 대한 정보가 연구에 제시 되는 정도를 결정할 수 있다는 점도 확인하였습니다.

본인은 면접하는 동안에 득정 질문에 대한 대답을 거부할 수 있으며, 언제든지 연구참 여를 취소할 수 있다고 들었습니다. 또한 연구참여자의 입장에서 궁금한 점을 연구자에게 언 제든지 질문할 기회가 있고, 연구자에게 납득할 수 있는 층분한 설명을 들을 수 있다는 점을 확인하였습니다.

연구참여자 :

연구자 :

연락처 : 010-2038-3724, taeyyun@gmail.com 
Interview Consent Form (translated into English)

\section{Research participation agreement (for participants/ storage)}

I voluntarily participated in this study. I understand the purpose and contents of the study and I accept to be interviewed.

I understand and agree that the contents of the researcher's conversation will be used as a research material through the recording. I can confirm the contents of the interview during the interview by checking the researcher's transcript and listen and agree that the recorded contents will be erased when the research is finished.

I have been told that during the interview I can refuse to answer certain questions and that I can cancel my participation at any time. We also have the opportunity to ask questions to the researcher at any time and to hear enough explanations that are understandable to the researcher. 
Lebenslauf

\section{Persönliche Daten}

Tae-Young Yun

Geburtsdatum: $\quad$ 03.03.1984

Geburtsort: $\quad$ Gimcheon/Südkorea

Staatsangehörigkeit: Südkoreanisch

\section{Schulausbildung}

1990 - 1996 Grundschule

1996 - 2002 Middle school / High school

\section{Studium und beruflicher Werdegang}

2002 - 2009 Studium (BBA und BA) im Doppelfach Betriebswirtschaft und soziale Arbeit an der Yonsei Universität in Seoul

July, 2009 National Health Insurance Research Institute in Korea, Field Practicum,

July, 2010 National Pension Research Institute in Korea, Field Practicum

2009 - 2011 Aufbaustudium (MSW) im Fach Sozialpolitik an der Yonsei Universität zum Thema "(A) Study on Development of Home-Based Service under Long-Term Care Insurance for the Elderly: focusing on care workers as a family member of beneficiaries"

2012 - 2019 Promotionsstudium an der Georg-August-Universität Göttingen bei Prof. Dr. Ilona Ostner 